\title{
Assessing outcomes of liver surgery : current status and future prospects
}

Citation for published version (APA):

van den Broek, M. A. J. (2013). Assessing outcomes of liver surgery : current status and future prospects. [Doctoral Thesis, Maastricht University]. Maastricht University. https://doi.org/10.26481/dis.20130328mb

Document status and date:

Published: 01/01/2013

DOI:

10.26481/dis.20130328mb

Document Version:

Publisher's PDF, also known as Version of record

\section{Please check the document version of this publication:}

- A submitted manuscript is the version of the article upon submission and before peer-review. There can be important differences between the submitted version and the official published version of record.

People interested in the research are advised to contact the author for the final version of the publication, or visit the DOI to the publisher's website.

- The final author version and the galley proof are versions of the publication after peer review.

- The final published version features the final layout of the paper including the volume, issue and page numbers.

Link to publication

\footnotetext{
General rights rights.

- You may freely distribute the URL identifying the publication in the public portal. please follow below link for the End User Agreement:

www.umlib.nl/taverne-license

Take down policy

If you believe that this document breaches copyright please contact us at:

repository@maastrichtuniversity.nl

providing details and we will investigate your claim.
}

Copyright and moral rights for the publications made accessible in the public portal are retained by the authors and/or other copyright owners and it is a condition of accessing publications that users recognise and abide by the legal requirements associated with these

- Users may download and print one copy of any publication from the public portal for the purpose of private study or research.

- You may not further distribute the material or use it for any profit-making activity or commercial gain

If the publication is distributed under the terms of Article $25 \mathrm{fa}$ of the Dutch Copyright Act, indicated by the "Taverne" license above, 


\section{Assessing outcomes of liver surgery:}

current status and future prospects

Maartje van den Broek 
Copyright: Maartje van den Broek, 's Gravenhage 2013

ISBN: 978-94-6169-363-1

Layout and printing: Optima Grafische Communicatie, Rotterdam, the Netherlands

This thesis is also available as an ePub (see www.e-pubs.nl/?epub=maartjevandenbroek).

Printing of this thesis was financially supported by ChipSoft BV, the Netherlands Society of Gastroenterology, Researchfonds Rijnland Ziekenhuis, and the Section of Experimental Gastroenterology of the Netherlands Society of Gastroenterology. 


\title{
Assessing outcomes of liver surgery: current status and future prospects
}

\author{
Proefschrift \\ ter verkrijging van de graad van doctor \\ aan de Universiteit Maastricht, \\ op gezag van de Rector Magnificus, prof. dr. LLG Soete, \\ volgens het besluit van het College van Decanen, \\ in het openbaar te verdedigen \\ op donderdag 28 maart 2013 om 14.00 uur
}

door

Maartje Adriana Jacoba van den Broek 


\section{Promotor}

Prof. dr. CHC Dejong

\section{Copromotor}

Dr. SWM Olde Damink

\section{Beoordelingscommissie}

Prof. dr. M van Kleef (voorzitter)

Prof. dr. M van Engeland

Prof. dr. O Farges (Beaujon Hospital, France)

Prof. dr. RRWJ van der Hulst

Prof. dr. RAEM Tollenaar (Leids Universitair Medisch Centrum) 


\section{Contents}

$\begin{array}{lll}\text { Chapter } 1 & \text { General introduction } & 7\end{array}$

Part I Assessing outcomes of liver surgery: critical appraisal of clinical endpoints

Chapter 2 Feasibility of randomized controlled trials in liver surgery using surgery-related mortality or morbidity as endpoint

Chapter 3 Proposal for uniform definitions of clinical outcomes of liver surgery: results of a web-based survey

Chapter 4 Development of a composite endpoint for randomized controlled trials in liver surgery

Part II Assessing outcomes of liver surgery: hepatic damage as a result of surgical technique

Chapter 5 Liver manipulation during liver surgery in humans is associated with hepatocellular damage and hepatic inflammation

Chapter 6 Randomized controlled trial analyzing the effect of 15 or 30 min intermittent Pringle maneuver on hepatocellular damage in man

Part III Assessing outcomes of liver surgery: hepatic damage as a result of oxaliplatin-based chemotherapy

Chapter 7 Nodular regenerative hyperplasia secondary to neoadjuvant chemotherapy for colorectal liver metastases

Chapter 8 Hyaluronic acid as a marker of hepatic sinusoidal obstruction syndrome secondary to oxaliplatin-based chemotherapy in patients with colorectal liver metastases

Chapter 9 Hepatic sinusoidal obstruction syndrome reduces the effect of oxaliplatin in patients with colorectal liver metastases

Chapter 10 The flavonoid monoHER prevents monocrotaline-induced hepatic sinusoidal injury in rats 
Chapter 11 Summary and general discussion 185

Chapter 12 Summary and general discussion (in Dutch) 199

$\begin{array}{lll}\text { Chapter } 13 \text { Clinical implications } & 213\end{array}$

$\begin{array}{llr}\text { Chapter } 14 & \text { Future perspectives } & 219\end{array}$

$\begin{array}{ll}\text { Appendices } & 227\end{array}$

Scientific output $\quad 255$

$\begin{array}{ll}\text { Dankwoord } & 261\end{array}$

$\begin{array}{lr}\text { Curriculum vitae } & 269\end{array}$ 


\section{Chapter 1}

\section{Liver failure after partial hepatic resection - A general introduction to the clinical problem}

Adapted from:

van den Broek MA, Olde Damink SW, Dejong $\mathrm{CH}$, Lang H, Malagó M, Jalan R, Saner

$\mathrm{FH}$. Liver failure after partial hepatic resection: definition, pathophysiology, risk factors and treatment. Liver Int 2008; 28: 767-80. 


\section{ABSTRACT}

Post-resectional liver failure (PLF) is a life-threatening complication that may arise after partial hepatic resection. In this chapter, the definition, incidence rate, pathogenesis, risk factors, risk assessment, prevention, clinical features, treatment and economic impact of PLF will be reviewed.

The reported incidence rates of PLF vary widely due to the lack of a uniform definition. Leaving the extremes out of consideration, the incidence rate of PLF ranges between 1 and 9 per cent. It is the leading cause of death after partial liver resection. An inadequate quantity or quality of residual liver mass are key events in the pathogenesis of PLF. Risk factors are either surgery or patient-related and include extensive resection of functional liver leaving small remnant liver mass and/or impaired liver function due to underlying liver disease. It is essential to identify patients at risk of PLF during the preoperative assessment, which includes evaluation of liver volume, anatomy and function. Preventive measures should be applied whenever possible as curative treatment options for PLF are limited. These preventive measures intend to increase remnant liver volume and protect remnant liver function. Patients suffering from PLF often develop multi-organ failure. Management principles focus upon support of end-organ and liver function. Due to the complex nature of the disease, the economic burden of PLF is high. 
Partial hepatic resection is the ultimate treatment for various benign and malignant liver tumours. In the last decade, outcomes of liver surgery have considerably improved, making a partial hepatic resection a relatively safe procedure ${ }^{1,2}$. The fact that partial hepatectomy is used in the setting of living donor liver transplantation as an accepted alternative to cadaveric donor liver transplantation may serve as an example $\mathrm{e}^{3,4}$.

The improved safety of liver surgery led to broadening of the indications for partial hepatic resection towards more extensive resections in high-risk patient groups. The resultant small remnant liver volume and pre-existent impaired liver function increase the risk of post-resectional liver failure (PLF). PLF is associated with major postoperative morbidity and mortality ${ }^{5,6}$. In this chapter, the definition, incidence rate, pathogenesis, risk factors, risk assessment, prevention, clinical features, treatment and economic impact of PLF will be reviewed.

\section{DEFINITION}

Up until recently, there was no uniformity concerning the definition of PLF, which made it difficult to interpret and compare results of clinical trials. In general, PLF was characterized as failure of one or more of the hepatic synthetic, detoxifying and excretory functions resulting in hypoalbuminemia, prolonged prothrombin time, hyperbilirubinemia, elevated serum lactate and/or hyperammonemia ${ }^{7-10}$. In 2011, the International Study Group of Liver Surgery proposed a standard definition of PLF (Table 1.1) ${ }^{11}$. This definition has been evaluated in a large patient cohort, but prospective, external validation is warranted.

Table 1.1 Consensus definition and severity grading of post-resectional liver failure by the International Study Group of Liver Surgery.

\begin{tabular}{ll}
\hline Definition & A postoperatively acquired deterioration in the ability of the liver (in patients with normal \\
& and abnormal liver function) to maintain its synthetic, excretory, and detoxifying functions, \\
characterized by an increased INR (or need for clotting factors to maintain a normal INR) and & hyperbilirubinemia (according to the normal cutoff levels defined by the local laboratory) on or \\
after postoperative day 5. If INR or serum bilirubin is increased preoperatively, PLF is defined by \\
an increasing INR (decreasing prothrombin index) and increasing serum bilirubin concentration \\
on or after postoperative day 5 (compared with the values of the previous day). Other obvious \\
causes for the observed biochemical and clinical alterations such as biliary obstruction should be \\
excluded \\
PLF resulting in abnormal laboratory parameters but requiring no change in the clinical \\
management of the patient \\
Grade B $\quad \begin{array}{l}\text { PLF resulting in a deviation from the regular clinical management but manageable without } \\
\text { invasive treatment }\end{array}$ \\
Grade C $\quad \begin{array}{l}\text { PLF resulting in a deviation from the regular clinical management and requiring invasive } \\
\text { treatment }\end{array}$
\end{tabular}

INR, international standardized ratio; PLF, post-resectional liver failure. 
In addition to this qualitative definition, PLF is quantitatively reasonably well defined by the so-called 50-50 criteria designed by Balzan and colleagues. In these 50-50 criteria, PLF is described as a prothrombin index below 50 per cent [equal to an international standardized ratio > 1.7] and serum bilirubin level of more than $50 \mu \mathrm{mol} / \mathrm{L}$ [2.9 mg/ $\mathrm{dL}$ ] on the $5^{\text {th }}$ postoperative day $(P O D)^{8}$. If the 50-50 criteria were met, patients had a significantly increased risk of mortality after liver surgery. A prospective follow-up study showed that these 50-50 criteria could serve as an independent prognostic factor of liver surgery-related death as early as POD $3^{6}$. Another group identified peak bilirubin above $120 \mu \mathrm{mol} / \mathrm{L}$ [7.0 mg/dL] as a simple, sensitive and specific cutoff value for the prediction of PLF-related death ${ }^{10}$.

All definitions of PLF are open to discussion. Their major drawback is that they cannot be applied in the early postoperative phase, when interventions that aim at preventing PLF may be most beneficial. Their shortcomings could be overcome by the prospective development of new definitions comprising sensitive biomarkers such as indocyanine green or ${ }^{13} \mathrm{C}$-methacetin kinetics ${ }^{12,13}$.

\section{INCIDENCE RATE}

The incidence of PLF in a case mix of patients ranges anywhere between 0 and 32 per cent (based on $1,2,7,8,10,14-29$ ). The highest incidence rates have been reported in specific groups, such as patients with cirrhosis of the liver, chemotherapy-associated hepatotoxicity or steatohepatitis ${ }^{30-33}$. Leaving the extremes out of consideration, its incidence varies between 1 and 9 per cent. The mortality rate in patients suffering from PLF is high ${ }^{10,33}$. Although the cause of death after partial hepatic resection is multifactorial, PLF seems to be the principal cause in 60 to 75 per cent of patients with lethal outcome ${ }^{10,19,33,34}$.

\section{PATHOGENESIS}

The resection of various amounts of functional liver mass induces both death and regeneration of remaining hepatocytes. Physiologically, regeneration outweighs hepatocyte death and both liver mass and function are rapidly restored ${ }^{35-37}$. For example, during the first ten days after right hepatectomy for living donor liver transplantation, liver mass restored from 39 up to 74 per cent of initial volume and liver biochemistry normalized rapidly ${ }^{35}$. This regeneration is triggered by an increased metabolic demand placed upon remnant hepatocytes (see for review ${ }^{38}$ ).

The pathogenesis of PLF is poorly understood and is thought to be related to an inadequate quality and/or quantity of remnant liver mass. The ability of the liver remnant 
to surmount the effect of surgical resection depends on its capacity to limit hepatocyte death, enhance its regenerative power, and resist metabolic stress while preserving an adequate function ${ }^{39,40}$. A variety of events can interfere with these protective processes. These events include hepatic parenchymal congestion, ischemia-reperfusion injury and postoperative infection ${ }^{41-43}$.

\section{Hepatic parenchymal congestion}

Partial hepatic resection leads to a relatively augmented sinusoidal perfusion, inducing shear-stress and congestion of hepatic parenchyma ${ }^{42}$. This results in vascular and parenchymal damage similar to the small-for-size syndrome after liver transplantation, although less severe ${ }^{44}$. Inadequate venous drainage of the liver remnant might additionally induce hepatic venous congestion and functional hepatic volume loss ${ }^{45}$.

\section{Hepatic ischemia-reperfusion injury}

Hepatic ischemia-reperfusion injury follows massive bleeding or hepatic in- and outflow occlusion during liver surgery. Although the resistance of the liver to warm ischemia is relatively high, hepatic ischemia and reperfusion activate a complex cascade that triggers the innate immune response and leads to cell death (see for review ${ }^{41}$ ). Although these processes are primarily intended to maintain homeostasis, unrestrained activation may become destructive.

\section{Infection}

Infection complicates the course of PLF either as a precipitant or during later stages ${ }^{9,33}$. Schindl and colleagues showed that the hepatic phagocytosis capacity decreased after partial liver resection with an S-shape correlation to the extent of hepatic resection ${ }^{43}$. Diminished hepatic clearance of bacteria after resection of large amounts of functional liver mass combined with an increased bacterial load following either bacterial translocation after inflow occlusion or intra-abdominal abscesses, enhance the susceptibility for the development of infection and PLF ${ }^{46}$.

\section{RISK FACTORS}

The most important, independent risk factors of PLF can be categorized as surgery or patient-related (Table 1.2). These factors include extensive liver resection, excessive intra-operative blood loss, prolonged operating time, male sex and advanced age $^{1,5,9,10,18,19}$. Also co-morbid conditions like diabetes mellitus as well as pre-existent liver diseases, such as steatohepatitis, cirrhosis, or chemotherapy-associated hepatotoxicity, predispose for the development of $\mathrm{PLF}^{31,47-50}$. At present, it is unclear if extra-hepatic 
Table 1.2 Risk factors of post-resectional liver failure.

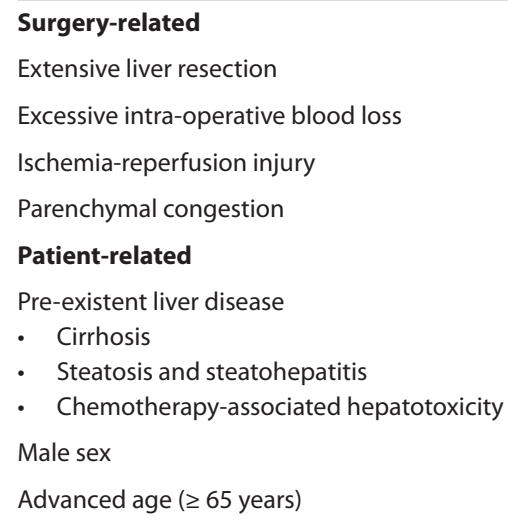

procedures, like concomitant biliary or vascular reconstructions, influence the incidence of PLF individually or by their association with extended liver resection, increased operating time and/or excessive blood loss.

\section{Surgery-related risk factors}

\section{Extensive liver resection}

The number of hepatic segments resected significantly correlates with postoperative complication rates (odds ratio (OR) 1.2 (95 per cent $\mathrm{Cl} 1.12$ to 1.29)) ${ }^{19}$. Remnant liver volume (RLV), defined as percentage remaining functional liver volume compared to preoperative functional liver volume, is regarded a reliable parameter to predict PLF and death, even more than the anatomic extent of the resection ${ }^{9,30,51}$. However, the exact amount of residual liver mass required to preserve sufficient liver function is unknown. In general, an RLV equal to or above 25 to 30 per cent in otherwise healthy livers is consistent with good post-resectional outcome 5, 9, 52. An RLV below 25 per cent in normal livers predicted PLF with a positive predictive value of 90 per cent ( 95 per cent $\mathrm{Cl} 68$ to 99 per cent) and a specificity of 98 per cent ( 95 per cent $\mathrm{Cl} 92$ to 100 per cent) ${ }^{5}$. When liver function is restricted, such as in patients with cirrhosis or chemotherapyassociated hepatotoxicity, RLV should be as high as 40 per cent to increase the likelihood of adequate remnant liver function ${ }^{18,52-54}$.

\section{Excessive intra-operative blood loss and blood transfusion}

Intra-operative blood loss and the need for blood transfusion predispose patients to PLF (OR 4.17 (95 per cent $\mathrm{Cl} 1.04$ to 17.5)) 18, 19,55. The cutoff point for an adverse outcome is suggested to be above 1000 - $1250 \mathrm{~mL}$ blood loss. Excessive blood loss leads to massive fluid shifts, which may induce bacterial translocation and systemic inflammation ${ }^{56}$. 
Massive bleeding also results in severe coagulopathy, which may predispose for intraabdominal hematoma and subsequent infection.

\section{Patient-related risk factors}

Male sex

Male sex doubles the propensity for developing PLF and post-resectional morbidity (OR 1.98 (95 per cent $\mathrm{Cl}$ not given)), consistent with earlier observations that males are more susceptible to develop postoperative complications $s^{5,10}$. Hypothetically, circulating sex hormones are responsible as testosterone is thought to exert an immunodepressive effect while estrogens exert an immunoprotective effect ${ }^{57}$.

\section{Advanced age}

Although data in literature are conflicting, advanced age (above 65 years) seems to predispose for PLF and post-resectional mortality (OR 1.8 (95 per cent $\mathrm{Cl} 0.78$ to 4.19)) ${ }^{8}$, especially after extended hepatic resections ${ }^{10,20,58}$. Elderly patients frequently suffer from co-morbid conditions and have a reduced regenerative capacity of hepatocytes ${ }^{59,60}$.

\section{Steatosis and steatohepatitis}

Subjects with elevated body mass index (above $30 \mathrm{~kg} / \mathrm{m}^{2}$ ) and subjects suffering from nonalcoholic fatty liver disease (NAFLD) experience significantly more complications after liver surgery compared to controls ${ }^{31,61-63}$. Patients with steatohepatitis, but not with simple hepatic steatosis, have a higher incidence of post-resectional hepatic decompensation compared to patients with healthy livers ( 16.7 versus 6.9 per cent, $p=0.049)^{63}$. The presence of NAFLD is hypothesized to be associated with an impaired hepatic microcirculation $^{64}$, decreased resistance to ischemia-reperfusion injury, increased intrahepatic oxidative stress and dysfunction in mitochondrial ATP-synthesis ${ }^{65}$. Animal studies report an impaired regeneration of steatotic livers, but the sparse clinical data currently available from living donors suffering from mild hepatic steatosis do not support these findings ${ }^{66,67}$.

\section{Cirrhosis}

The incidence of PLF after partial hepatic resection in patients with cirrhosis ranges between 5 and 10 per cent taking into account a higher number of restrictive surgical procedures performed in this subgroup $30,47,49$. The high risk of developing PLF in patients with cirrhosis can be explained by the wide range of co-morbid conditions like portal hypertension ${ }^{68}$, diabetes mellitus, jaundice ${ }^{69}$, malnutrition, hypersplenism and coagulopathy as well as frequently impaired preoperative liver function and hepatic functional reserve ${ }^{70}$. Furthermore, patients with cirrhosis seem to have an impaired hepatic regenerative capacity ${ }^{37}$. 


\section{Chemotherapy-associated hepatotoxicity}

Perioperative, systemic chemotherapy regimens improve progression-free survival in patients with colorectal liver metastases (CLMs) and have therefore become standard of care in this patient group ${ }^{71}$. However, they are associated with an increased incidence of PLF and PLF-related death as a result of chemotherapy-associated hepatotoxicity ${ }^{32,71-73}$. Oxaliplatin induces sinusoidal injury of the non-tumour-bearing liver (the so-called sinusoidal obstruction syndrome (SOS)) in approximately 3 out of 5 patients ${ }^{74,75}$. This sinusoidal injury is thought to reduce the regenerative capacity of the liver remnant ${ }^{76,77}$. Treatment with irinotecan is associated with an increased risk of steatohepatitis (so-called chemotherapy associated steatohepatitis (CASH)), which increases 90-day mortality ${ }^{32,78}$.

\section{RISK ASSESSMENT}

Preoperative risk assessment should ideally consist of four features including clinical, biochemical, volumetric and functional data (see for review ${ }^{79}$ ). An assessment focussing on only one of these aspects is regarded not to be useful. A thorough evaluation of risk factors is generally believed to enable the selection of candidates suitable for a safe partial hepatic resection with low risk of PLF. However, the onset of PLF will remain unpredictable in a subset of patients.

\section{Assessment of clinical condition}

The identification of co-morbid conditions like obesity, diabetes mellitus, malnutrition, cardiovascular, pulmonary, hepatic or renal disease is pivotal as they increase the susceptibility for major complications, even if hepatic functional reserve is adequate ${ }^{7,50}$. Additionally, the existence of portal hypertension must be objectified, as this elevates the risk of bleeding and $\mathrm{PLF}^{68}$.

\section{Assessment of biochemical parameters}

Tests analyzing hepatic synthetic (serum albumin and clotting factors) or excretory function (serum bilirubin) are non-specific for the assessment of hepatic function and do not correlate with post-resectional outcomes; however, they may indicate hepatic dysfunction ${ }^{70,80}$. Furthermore, serum activities of transaminases as well as alkaline phosphatase and $\gamma$-glutamyl-transferase are non-specific for the evaluation of hepatic function, but can signal hepatocyte necrosis, increased hepatitic activity or the presence of cholestasis. 


\section{Assessment of liver anatomy and volumetry}

Standard liver resection planning is based on contrast-enhanced 2D computed tomography $(C T)$ or magnetic resonance imaging $(\mathrm{MRI})$, supplemented with intra-operative ultrasonography. Based on these imaging techniques, information on the anatomy of liver segments, biliary structures, hepatic vasculature and tumour localization can be extracted. However, 2D CT supplies only marginal data about the distribution pattern of hepatic venous in- and outflow related to hepatic segments and tumour location ${ }^{81}$. In this context, 3D CT reconstructions have proven to deliver useful additional information in selected patients, for example those requiring an extended hepatic resection ${ }^{82,83}$.

Ultrasonography, CT and MRI are also able to provide information on the quality of hepatic parenchyma in patients with suspected underlying liver disease, such as hepatic steatosis or chemotherapy-associated hepatotoxicity ${ }^{84,85}$. When pre-existent liver disease is expected and imaging studies are inconclusive, a (transjugular) biopsy of the non-tumorous liver or even a diagnostic laparoscopy may be helpful to establish the diagnosis.

Volumetric analysis can be performed using CT imaging, which provides good quality data on total, functional (i.e. total liver volume minus tumour volume) and remnant liver volume ${ }^{86,87}$. Appropriate formulas combining body surface area and weight are available for the calculation of total liver volume and these formulas are hypothesized to reflect the metabolic demands more exactly than CT volumetry alone ${ }^{88}$.

\section{Assessment of liver function}

Assessment of liver function is useful to determine hepatic functional reserve. Several dynamic tests quantitatively evaluate liver function, among which the indocyanine green retention at 15 minutes post intravenous injection (ICGR15), the galactose elimination test, the lidocaine-monoethylglycine-xylidide test (MEGX), the ${ }^{13} \mathrm{C}$-methacetin test (LiMAx) and the ${ }^{14} \mathrm{C}$-aminopyrine breath test are most frequently used. They all assess hepatic clearance or conversion of xenobiotics.

ICGR15 depends upon hepatic perfusion rate and subjects with an ICGR15 above 15 to 20 per cent are generally believed to have an impaired hepatic functional reserve ${ }^{23,47,89}$. Recently, ICGR15 above 10 per cent was found to be a reliable predictor for the presence of sinusoidal injury secondary to neoadjuvant chemotherapy treatment in patients with $\mathrm{CLMs}^{72}$.

The hepatic cytosolic capacity is reflected by the galactose elimination test and the critical value is considered to be elimination of less than $6 \mathrm{mg} / \mathrm{min} / \mathrm{kg}$ in patients without and less than $4 \mathrm{mg} / \mathrm{min} / \mathrm{kg}$ in patients with hepatocellular carcinoma ${ }^{90}$.

The MEGX test, LiMAx test and ${ }^{14} \mathrm{C}$-aminopyrine breath test are based on the rate of metabolite formation of specific drugs. MEGX and LiMAx tests both reflect the conversion rate of a prodrug by hepatic cytochrome P450. For MEGX, a value equal to or below 
$25 \mu \mathrm{g} / \mathrm{L}$ was related to PLF in patients with cirrhosis ${ }^{91}$. A LiMAx cutoff value of $85 \mu \mathrm{g} / \mathrm{kg} /$ hr on POD1 had a sensitivity of 100 per cent and specificity of 94 per cent for identifying patients developing $\mathrm{PLF}^{12}$. Finally, the aminopyrine breath test evaluates the hepatic oxidative function by measurement of ${ }^{14} \mathrm{CO}_{2}$ exhalation. The normal value is an exhalation of 7 per cent ${ }^{14} \mathrm{CO}_{2}$ and the critical value seems to be below 2.3 per cent ${ }^{92,93}$. There is no consensus regarding the validity of a single test for assessment of liver function and hepatic functional reserve in operative planning.

\section{Assessment of liver function in patients with cirrhosis}

For patients with cirrhosis, scoring systems used to assess the feasibility of partial hepatic resection are the Child-Pugh score and the model for end-stage liver disease (MELD) score ${ }^{7,94,95}$. As they both are designed for other purposes, their validity to predict postresectional outcome has only recently been established and results are inconsistent. In general, Child-Pugh class $C$ is considered an absolute contra-indication for surgery and class $\mathrm{B}$ permits only minor liver resection ${ }^{96}$. Schroeder and colleagues reported superiority of the Child-Pugh to MELD score in predicting short-term morbidity and mortality after partial hepatic resection 7 . However, other authors stated that preoperative MELD score was a highly reliable predictor in certain subgroups. For example, a MELD score above 11 in patients with cirrhosis could predict PLF accurately (area under receiver operating characteristic curve 0.92 (95 per cent $\mathrm{Cl} 0.87$ to 0.96$))^{97}$.

\section{PREVENTION}

As curative treatment options for PLF are limited, prevention of this life-threatening condition is essential.

\section{Surgery-related risk factors}

\section{Extensive liver resection}

Portal vein occlusion and/or two-stage hepatectomy can be considered in case of expected small RLV. Portal vein occlusion by either embolization or ligation of one of the portal veins is advised in patients with normal liver function if RLV is estimated to be below 25 to 30 per cent or in patients with impaired liver function (reflected by an IGCR15 between 15 to 20 per cent) and estimated RLV below 40 to 45 per cent ${ }^{98-100}$. Its effectiveness depends on the severity of pre-existent liver disease and co-morbid conditions, ranging from 20 to 46 per cent volume increase after 2 to 4 weeks ${ }^{77,99,100}$. Portal vein embolization increased the feasibility of partial hepatectomy by 19 per cent ${ }^{99}$, and had a complication rate between 9 to 13 per cent (see for review ${ }^{98}$ ). It is hypothesized 
to promote intra-hepatic tumour growth in the non-embolized liver, but this does not seem to have an effect on long-term outcomes of partial hepatic resection for CLMs ${ }^{101}$. Interval chemotherapy might be beneficial, though, seems to negatively affect hypertrophy ratio ${ }^{77}$. A combination of portal vein occlusion and in situ split liver transection with preservation of arterial inflow and venous outflow (the ALPPS approach) has recently been proven to induce a 40 to 80 per cent hypertrophy of the future liver remnant in only 6 days, which was remarkably greater than the hypertrophy observed by portal vein occlusion alone ${ }^{102}$. Stem cell therapy as adjunct to portal vein occlusion seems promising, but robust clinical evidence for its effectiveness is lacking to date ${ }^{103}$.

Two-stage hepatectomy utilizes the regenerative capacity of the liver. Studies focussing on the feasibility of two-stage hepatectomy combined with neoadjuvant chemotherapy and/or portal vein occlusion reported success rates of 70 to 81 per cent along with an increase in median survival time compared to palliative chemotherapy alone in patients with initially unresectable $\mathrm{CLMs}^{77,104,105}$ (see for review ${ }^{106}$ ).

\section{Excessive intra-operative blood loss and blood transfusion}

Lowering central venous pressure during dissection to below $5 \mathrm{cmH}_{2} \mathrm{O}$ limits intraoperative blood loss without deterioration of renal function ${ }^{107,108}$. A combination of the former with portal triad clamping or total vascular exclusion is most advantageous for the prevention of excessive intra-operative blood loss ${ }^{109}$. The latter procedures both induce hepatic ischemia-reperfusion injury, but total vascular exclusion leads to more important hemodynamic changes and higher complication rates compared to hepatic inflow occlusion alone ${ }^{110}$. With respect to hepatic inflow occlusion, intermittent portal triad clamping is regarded superior to continuous clamping ${ }^{111}$. However, the optimal ischemic intervals are unknown. Ischemic preconditioning (IPC) before portal triad clamping reduces hepatocyte damage in a murine as well as a human model of hepatic inflow occlusion ${ }^{65}, 112-115$. Petrowsky and colleagues reported that IPC combined with continuous portal triad clamping was as effective as intermittent clamping in noncirrhotic livers, though intermittent clamping was accompanied by significantly higher intra-operative blood loss, transfusion requirement and operating time ${ }^{116}$. The latter study also showed that the protective effect of IPC seemed to diminish in patients above 65 years of age. Consequently, intermittent clamping is suggested to be superior to IPC combined with continuous clamping in elderly patients ${ }^{116}$.

\section{Patient-related risk factors}

\section{Steatosis and steatohepatitis}

Data from living liver donors suffering from biopsy-proven moderate steatosis revealed that a body weight reduction of 5 per cent or intervention with low-fat, high-protein 
diet and exercise significantly improved hepatic steatosis ${ }^{117,118}$. In addition, drugs that are able to reverse NAFLD have become available recently ${ }^{119}$. The beneficial effects of preoperative voluntary weight loss or drug therapy on post-resectional outcomes in patients with NAFLD scheduled to undergo liver surgery have not been studied to date.

\section{Cirrhosis}

Approximately 65 to 90 per cent of patients with advanced liver disease suffer from protein-calorie malnutrition ${ }^{120}$. It has therefore been hypothesized that the nutritional status of these patients should be corrected prior to surgery. Indeed, a beneficial effect of additional parenteral nutrition has been demonstrated in patients with cirrhosis undergoing major hepatectomy ${ }^{121}$.

\section{Chemotherapy-associated hepatotoxicity}

Optimizing the interval between neoadjuvant chemotherapy treatment and hepatic resection might reduce the risk of postoperative complications, as a longer interval between chemotherapy and surgical resection seemed to decrease the incidence of sinusoidal injury ${ }^{72,122}$. Addition of bevacizumab, a monoclonal antibody against vascular endothelial growth factor, to conventional chemotherapeutic agents also seemed to protect against the development of SOS in patients with CLMs, as did oral aspirin treatment ${ }^{123-125}$.

\section{MANIFESTATION}

The majority of patients with PLF do not only suffer from liver failure, but also meet the criteria for the systemic inflammatory response syndrome and multi-organ failure.

\section{Liver}

The clinical consequences of PLF are jaundice, coagulopathy, ascites, oedema and/or hepatic encephalopathy (HE). Data on liver function on different days after uncomplicated hepatic resection showed temporal liver dysfunction reflected by initially increased serum bilirubin levels and increased prothrombin times before normalization of these values on POD7-10,35,36. However, when prothrombin index dropped below 50 per cent and serum bilirubin level exceeded $50 \mu \mathrm{mol} / \mathrm{L}[2.9 \mathrm{mg} / \mathrm{dL}]$ on either POD3 or POD5, the risk of PLF and PLF-related mortality increased significantly ${ }^{6,8}$.

\section{Circulation}

Circulatory failure occurring during PLF resembles the circulatory failure of patients with sepsis ${ }^{126}$. The pathophysiological changes usually observed are enhanced vascular 
permeability, diffuse intravascular coagulation and peripheral vasodilatation, which are clinically represented by reduced peripheral resistance leading to hemodynamic instability.

\section{Lungs}

Although moderate pulmonary oedema is a normal finding after partial hepatic resection due to general hemodynamic alterations, this does usually not impair oxygen exchange ${ }^{127}$. Severe remote lung injury, pulmonary oedema and acute respiratory distress syndrome can develop as part of multi-organ failure accompanying PLF.

\section{Kidneys}

Post-resectional renal dysfunction can either result from perioperative disturbances in renal circulation inducing acute tubular necrosis or can accompany PLF ${ }^{107,{ }^{128}}$. It is characterized by an increase in creatinine level, uremia or oliguria and may cause ascites formation, pleural effusion and fluid overload requiring diuretics or hemofiltration ${ }^{129}$. There is a distinct chance of reversibility of renal failure when there is recovery of PLF. It can be hypothesized that renal failure interferes with the pivotal role of the kidney in ammonia excretion, leading to hyperammonemia and HE in patients suffering from PLF $^{130}$.

\section{Brain}

Patients with PLF may suffer from HE which is a potentially reversible neuropsychiatric disorder characterized by varying degrees of confusion and disorientation ${ }^{131}$. Hyperammonemia plays a central role in the development of $\mathrm{HE}^{132}$. There seems to be a strong association between infection and the development of $\mathrm{HE}$, as inflammation makes the brain more vulnerable to ammonia ${ }^{133}$. Data concerning the incidence of HE after partial hepatic resection are sparse.

\section{TREATMENT}

Although the mortality rate of PLF is high, it is a potentially reversible disorder because of the regenerative capacity of the liver remnant. Large, randomized controlled trials (RCTs) concerning the treatment of PLF are lacking and therefore, recommendations for its treatment based on high level of evidence research are difficult to make. Management principles resemble those applied to patients with acute liver failure, acute-on-chronic liver failure and sepsis and focus on support of end-organ and liver function ${ }^{134,135}$. In desperate situations, rescue hepatectomy and subsequent liver transplantation may be considered. 


\section{Support of end-organ function}

Goal-directed therapy should be provided for circulatory disturbances, renal and pulmonary dysfunction, coagulopathy, malnutrition and HE (Table 1.3). As there seems to be a strong link between infection and PLF, frequent cultures for bacteria and fungi are important ${ }^{33}$. The use of prophylactic antibiotics after partial hepatectomy for the prevention of infectious complications is not supported by evidence from literature ${ }^{136,137}$. However, the administration of antibiotics in patients suffering from acute liver failure unrelated to surgery is associated with a significant decrease in infectious complications and this may also be advantageous in patients at high risk of PLF ${ }^{138}$.

Table 1.3 Treatment goals of patients suffering from post-resectional liver failure.

\begin{tabular}{ll}
\hline System & Goal \\
\hline Circulation & Central venous pressure between $8-12 \mathrm{mmHg}$ \\
& Mean arterial pressure between $65-90 \mathrm{mmHg}$ \\
& Hematocrit $\geq 30$ per cent \\
& Pulmonary capillary wedge pressure $\leq 12-15 \mathrm{mmHg}$ \\
& Urine output $\geq 0.5 \mathrm{~mL} / \mathrm{kg} / \mathrm{hr}$ \\
Kidneys & Arterial oxygen saturation $\geq 93$ per cent \\
Lungs & Central venous oxygen saturation $\geq 70$ per cent \\
& Improvement of HE to grade $\leq 2$ \\
Brain & In case of bleeding: \\
Coagulation & $-\quad$ Platelet count $\geq 5010^{9} / \mathrm{L}$ \\
& $-\quad$ INR $\leq 1.5$ \\
Nutrition & Enteral energy supply of $2000 \mathrm{kcal} /$ day \\
\hline
\end{tabular}

$\mathrm{HE}$, hepatic encephalopathy; INR, international standardized ratio.

\section{Support of liver function}

Support of liver function can be achieved by cell-based therapy or extracorporeal liver support systems. The rationale of cell therapy is to replace defective cells in order to substitute organ function. The use of hepatocyte or stem cell transplantation for the treatment of PLF seems promising, but scientific evidence for its effectiveness in man is lacking to date ${ }^{139}$.

Extracorporeal liver support systems aim to replace the detoxifying or synthetic functions of the failing liver. A recent meta-analysis demonstrated a survival advantage of extracorporeal liver support devices in patients suffering from acute liver failure unrelated to surgery ${ }^{140}$. RCTs analyzing the effect of extracorporeal liver support systems in patients with PLF are lacking, but some non-controlled studies have been conducted on its effectiveness. 


\section{Plasma exchange}

Plasma exchange is an extracorporeal supportive procedure in which plasma is separated from blood cells and treated or substituted with fresh frozen plasma. This technique supplies plasma components that are lacking (e.g. albumin and clotting factors) and removes water-soluble toxins related to $\mathrm{HE}$ (e.g. ammonia) hereby improving the clinical condition of patients suffering from PLF ${ }^{141,142}$.

\section{$M{ }^{\circledR} S^{\circledR}$}

The molecular absorbent recirculating system (MARS ${ }^{\circledR}$, Gambro, Lund, Sweden) removes water-soluble along with albumin-bound toxins from the plasma by means of dialyzing blood against an albumin-containing dialysate across an albumin-impregnated membrane ${ }^{143,144}$. Promising results have been shown when applied during acute liver failure or acute-on-chronic liver failure ${ }^{145}$, but the use of MARS for treatment of PLF has only been validated in small, uncontrolled and non-randomized trials. Unfortunately, MARS treatment for PLF and progressive septic multi-organ failure did not positively affect patient survival ${ }^{146-148}$.

\section{Prometheus $^{\circledR}$}

Prometheus $^{\circledast}$ (Fresenius Medical Care, St Wendel, Germany) uses the principle of fractionated plasma separation and adsorption for removal of water-soluble along with albumin-bound toxins. Albumin-bound toxins pass an albumin-permeable membrane and native albumin is subsequently detoxified by adsorption, after which the cleansed albumin is returned to the patient ${ }^{143}{ }^{144}$. The detoxifying capacity of Prometheus appeared to be superior to that of MARS when applied during acute-on-chronic liver failure ${ }^{149}$, but no clinical survival benefit has been proven yet. Studies on the application of Prometheus for PLF are lacking.

\section{Bioartificial liver and the extracorporeal liver assist device}

Bioartificial liver supporting systems using cryopreserved xenogenic or human hepatocytes have been validated in one large, prospective controlled trial for acute liver failure and primary non-function after liver transplantation. Results are promising as the application is safe, but survival only significantly improved for patients with acute liver failure ${ }^{150}$. Again, data on the application of these bioartificial liver supporting systems for the treatment of PLF in man are lacking.

\section{Rescue hepatectomy and liver transplantation}

The use of a rescue hepatectomy followed by liver transplantation (LTX) is based on the concept that the necrotic, failing liver is the source of unknown humoral substances that contribute to the systemic inflammatory response syndrome in patients with liver 
failure ${ }^{151}$. LTx is controversial in patients suffering from liver failure after partial hepatectomy for malignant disease and only two trials describe outcomes of LTx for PLF ${ }^{152,153}$. Otsuka and colleagues showed that LTx was associated with considerable morbidity, but mean survival time was prolonged from 1.4 to 42.2 months. All patients $(n=4)$ that suffered from PLF but were not appropriate candidates for LTx died, while those undergoing orthotopic LTx survived $(n=7)^{153}$. However, no criteria are available for the selection of patients that will benefit from emergency LTx for PLF.

\section{ECONOMIC IMPACT}

Due to the complex nature of the disease, patients with PLF will need intensive treatment. A study performed in a tertiary referral centre in Germany in 2009 showed that there was a significant difference between the hospital costs of patients with regular recovery after liver surgery (Euro 25,980) versus patients suffering from PLF (Euro 82,199; $p=0.013$ ), with a shift of costs towards intensive care ${ }^{154}$. The group of patients suffering from PLF represented 17 per cent of the population, but was responsible for 39 per cent of total hospital costs. These numbers show that PLF is not only a significant clinical, but also economic problem.

\section{RATIONALE OF THE CURRENT THESIS}

In the general introduction, the current state of knowledge on liver failure after partial hepatic resection is presented. Although the incidence rate of PLF is relatively low, it is one of the most severe complications of liver surgery and it is the single most important cause of postoperative death. Preventing PLF or improving the outcome of patients suffering from PLF is therefore important in offering potentially curative treatment to patients with liver tumours. Obviously, information on ways to improve outcomes of patients suffering from liver failure after partial hepatectomy would best be derived from high level of evidence research such as RCTs. Unfortunately, such evidence does hitherto not exist and RCTs using PLF as primary endpoint are difficult to perform. The latter is due to the low incidence of PLF, which makes it practically impossible to conduct adequately powered trials using a single component, clinical endpoint as primary endpoint. We therefore investigated ways to facilitate the conduct of high level of evidence research on improving outcomes of patients undergoing liver surgery by using broadly accepted composite and surrogate endpoints. 


\section{AIMS OF THE CURRENT THESIS}

The broader aim of this thesis was to assess and improve outcomes of patients undergoing liver surgery. This then translated into three more specific aims looking at (1) the feasibility of RCTs in liver surgery using clinical endpoints, (2) surgery-related risk factors of PLF and (3) patient-related risk factors of PLF. Taken together, the aims of the present thesis were as follows:

Aim 1: to develop strategies that increase the feasibility of conducting RCTs in liver surgery using clinical outcomes as primary endpoints (Chapter 2, 3 and 4).

Aim 2: to evaluate the effect of various surgical techniques on hepatic damage in patients undergoing liver surgery (Chapter 5 and 6).

Aim 3: to study the effect of oxaliplatin-based chemotherapy on hepatic damage in patients undergoing partial liver resection for CLMs (Chapter 7, 8, 9 and 10).

\section{OUTLINE OF THE CURRENT THESIS}

The present thesis consists of four major parts. In the first part, reasons for the shortage of RCTs in liver surgery are explored and solutions are being offered. Chapter 2 examines the feasibility of RCTs in liver surgery using surgery-related mortality or morbidity as primary endpoint. In chapter 3 , standard definitions of liver surgery-related outcomes to be used as clinical endpoints of RCTs in liver surgery are proposed. Chapter 4 describes the development of a liver surgery specific composite endpoint, which can be used as a primary endpoint of future RCTs.

The second part of this thesis focuses on the effect of two common surgical techniques on liver cell damage in patients undergoing liver surgery. Chapter 5 deals with the association between mobilization of the liver and hepatocellular damage and hepatic inflammation in humans. In chapter 6, an RCT analyzing the effect of 15 or 30 min intermittent Pringle maneuver on liver cell damage during liver surgery in humans is described.

Part III focuses on hepatic damage secondary to oxaliplatin-based chemotherapy in patients with CLMs. Chapter 7 illustrates the clinical consequence of hepatic sinusoidal injury in patients treated with oxaliplatin prior to liver surgery for CLMs. Chapter 8 explores the value of systemic hyaluronic acid level as preoperative, non-invasive marker of SOS. The impact of sinusoidal injury of the non-tumour-bearing liver on tumour regression grade is examined in chapter 9 . Chapter 10 analyzes the preventive effect of the flavonoid monoHER on hepatocellular and sinusoidal injury in an experimental rat model. 
The fourth part of this thesis encompasses a summary and discussion of the findings described in the first three parts (chapter 11 and 12). In chapter 13 and 14, clinical implications and directions for the future are emphasized. 


\section{REFERENCES}

1. Poon RT, Fan ST, Lo CM, Liu CL, Lam CM, Yuen WK, et al. Improving perioperative outcome expands the role of hepatectomy in management of benign and malignant hepatobiliary diseases: analysis of 1222 consecutive patients from a prospective database. Ann Surg 2004; 240: 698-708.

2. Dimick JB, Wainess RM, Cowan JA, Upchurch GR, Jr., Knol JA, Colletti LM. National trends in the use and outcomes of hepatic resection. J Am Coll Surg 2004; 199: 31-8.

3. Belghiti J. The first anatomical right resection announcing liver donation. J Hepato/ 2003; 39: 4759.

4. Broelsch CE, Malago M, Testa G, Valentin Gamazo C. Living donor liver transplantation in adults: outcome in Europe. Liver Transpl 2000; 6: S64-5.

5. Shoup M, Gonen M, D'Angelica M, Jarnagin WR, DeMatteo RP, Schwartz LH, et al. Volumetric analysis predicts hepatic dysfunction in patients undergoing major liver resection. $J$ Gastrointest Surg 2003; 7: 325-30.

6. Paugam-Burtz C, Janny S, Delefosse D, Dahmani S, Dondero F, Mantz J, et al. Prospective validation of the "fifty-fifty" criteria as an early and accurate predictor of death after liver resection in intensive care unit patients. Ann Surg 2009; 249: 124-8.

7. Schroeder RA, Marroquin CE, Bute BP, Khuri S, Henderson WG, Kuo PC. Predictive indices of morbidity and mortality after liver resection. Ann Surg 2006; 243: 373-9.

8. Balzan S, Belghiti J, Farges O, Ogata S, Sauvanet A, Delefosse D, et al. The "50-50 criteria" on postoperative day 5: an accurate predictor of liver failure and death after hepatectomy. Ann Surg 2005; 242: 824-8.

9. SchindI MJ, Redhead DN, Fearon KC, Garden OJ, Wigmore SJ. The value of residual liver volume as a predictor of hepatic dysfunction and infection after major liver resection. Gut 2005; 54: 289-96.

10. Mullen JT, Ribero D, Reddy SK, Donadon M, Zorzi D, Gautam S, et al. Hepatic insufficiency and mortality in 1,059 noncirrhotic patients undergoing major hepatectomy. J Am Coll Surg 2007; 204: 854-62.

11. Rahbari NN, Garden OJ, Padbury R, Brooke-Smith M, Crawford M, Adam R, et al. Posthepatectomy liver failure: a definition and grading by the International Study Group of Liver Surgery (ISGLS). Surgery 2011; 149: 713-24.

12. Stockmann M, Lock JF, Riecke B, Heyne K, Martus P, Fricke M, et al. Prediction of postoperative outcome after hepatectomy with a new bedside test for maximal liver function capacity. Ann Surg 2009; 250: 119-25.

13. Sugimoto $\mathrm{H}$, Okochi $\mathrm{O}$, Hirota M, Kanazumi N, Nomoto S, Inoue S, et al. Early detection of liver failure after hepatectomy by indocyanine green elimination rate measured by pulse dye-densitometry. J Hepatobiliary Pancreat Surg 2006; 13: 543-8.

14. Virani S, Michaelson JS, Hutter MM, Lancaster RT, Warshaw AL, Henderson WG, et al. Morbidity and mortality after liver resection: results of the patient safety in surgery study. J Am Coll Surg 2007; 204: 1284-92.

15. McCall J, Koea J, Gunn K, Rodgers M, Jarvis J. Liver resections in Auckland 1998-2001: mortality, morbidity and blood product use. N Z Med J 2001; 114: 516-9.

16. Benzoni E, Lorenzin D, Baccarani U, Adani GL, Favero A, Cojutti A, et al. Resective surgery for liver tumor: a multivariate analysis of causes and risk factors linked to postoperative complications. Hepatobiliary Pancreat Dis Int 2006; 5: 526-33.

17. Coelho JC, Claus CM, Machuca TN, Sobottka WH, Goncalves CG. Liver resection: 10-year experience from a single institution. Arq Gastroenterol 2004; 41: 229-33. 
18. Imamura H, Seyama Y, Kokudo N, Maema A, Sugawara Y, Sano K, et al. One thousand fifty-six hepatectomies without mortality in 8 years. Arch Surg 2003; 138: 1198-206.

19. Jarnagin WR, Gonen M, Fong Y, DeMatteo RP, Ben-Porat L, Little S, et al. Improvement in perioperative outcome after hepatic resection: analysis of 1,803 consecutive cases over the past decade. Ann Surg 2002; 236: 397-406.

20. Alfieri S, Carriero C, Caprino P, Di Giorgio A, Sgadari A, Crucitti F, et al. Avoiding early postoperative complications in liver surgery. A multivariate analysis of 254 patients consecutively observed. Dig Liver Dis 2001; 33: 341-6.

21. Akashi K, Mizuno S, Isaji S. Comparative study of perioperative management of hepatic resection. Dig Dis Sci 2000; 45: 1988-95.

22. Belghiti J, Hiramatsu K, Benoist S, Massault P, Sauvanet A, Farges O. Seven hundred forty-seven hepatectomies in the 1990s: an update to evaluate the actual risk of liver resection. J Am Coll Surg 2000; 191: 38-46.

23. Das BC, Isaji S, Kawarada Y. Analysis of 100 consecutive hepatectomies: risk factors in patients with liver cirrhosis or obstructive jaundice. World J Surg 2001; 25: 266-72.

24. Brancatisano R, Isla A, Habib N. Is radical hepatic surgery safe? Am J Surg 1998; 175: 161-3.

25. Finch MD, Crosbie JL, Currie E, Garden OJ. An 8-year experience of hepatic resection: indications and outcome. Br J Surg 1998; 85: 315-9.

26. Taniguchi H, Takahashi T. Analysis of 210 elective hepatic resections. Hepatogastroenterology 1997; 44: 1624-31.

27. Rees M, Plant G, Wells J, Bygrave S. One hundred and fifty hepatic resections: evolution of technique towards bloodless surgery. Br J Surg 1996; 83: 1526-9.

28. Sun HC, Qin LX, Wang L, Ye QH, Wu ZQ, Fan J, et al. Risk factors for postoperative complications after liver resection. Hepatobiliary Pancreat Dis Int 2005; 4: 370-4.

29. Buell JF, Rosen S, Yoshida A, Labow D, Limsrichamrern S, Cronin DC, et al. Hepatic resection: effective treatment for primary and secondary tumors. Surgery 2000; 128: 686-93.

30. Shirabe K, Shimada M, Gion T, Hasegawa H, Takenaka K, Utsunomiya T, et al. Postoperative liver failure after major hepatic resection for hepatocellular carcinoma in the modern era with special reference to remnant liver volume. J Am Coll Surg 1999; 188: 304-9.

31. Behrns KE, Tsiotos GG, DeSouza NF, Krishna MK, Ludwig J, Nagorney DM. Hepatic steatosis as a potential risk factor for major hepatic resection. J Gastrointest Surg 1998; 2: 292-8.

32. Vauthey JN, Pawlik TM, Ribero D, Wu TT, Zorzi D, Hoff PM, et al. Chemotherapy regimen predicts steatohepatitis and an increase in 90-day mortality after surgery for hepatic colorectal metastases. J Clin Oncol 2006; 24: 2065-72.

33. Capussotti L, Vigano L, Giuliante F, Ferrero A, Giovannini I, Nuzzo G. Liver dysfunction and sepsis determine operative mortality after liver resection. Br J Surg 2009; 96: 88-94.

34. Detroz B, Sugarbaker PH, Knol JA, Petrelli N, Hughes KS. Causes of death in patients undergoing liver surgery. Cancer Treat Res 1994; 69: 241-57.

35. Nadalin S, Testa G, Malago M, Beste M, Frilling A, Schroeder T, et al. Volumetric and functional recovery of the liver after right hepatectomy for living donation. Liver Transp/ 2004; 10: 1024-9.

36. Suc B, Panis Y, Belghiti J, Fekete F. 'Natural history' of hepatectomy. Br J Surg 1992; 79: 39-42.

37. Yamanaka N, Okamoto E, Kawamura E, Kato T, Oriyama T, Fujimoto J, et al. Dynamics of normal and injured human liver regeneration after hepatectomy as assessed on the basis of computed tomography and liver function. Hepatology 1993; 18: 79-85.

38. Michalopoulos GK, DeFrances MC. Liver regeneration. Science 1997; 276: 60-6. 
39. Morita T, Togo S, Kubota T, Kamimukai N, Nishizuka I, Kobayashi T, et al. Mechanism of postoperative liver failure after excessive hepatectomy investigated using a cDNA microarray. J Hepatobiliary Pancreat Surg 2002; 9: 352-9.

40. Takeda K, Togo S, Kunihiro O, Fujii Y, Kurosawa H, Tanaka K, et al. Clinicohistological features of liver failure after excessive hepatectomy. Hepatogastroenterology 2002; 49: 354-8.

41. Jaeschke H. Molecular mechanisms of hepatic ischemia-reperfusion injury and preconditioning. Am J Physiol Gastrointest Liver Physiol 2003; 284: G15-26.

42. Kin Y, Nimura Y, Hayakawa N, Kamiya J, Kondo S, Nagino M, et al. Doppler analysis of hepatic blood flow predicts liver dysfunction after major hepatectomy. World J Surg 1994; 18: 143-9.

43. SchindI MJ, Millar AM, Redhead DN, Fearon KC, Ross JA, Dejong CH, et al. The adaptive response of the reticuloendothelial system to major liver resection in humans. Ann Surg 2006; 243: 507-14.

44. Palmes D, Budny TB, Dietl KH, Herbst H, Stratmann U, Spiegel HU. Detrimental effect of sinusoidal overperfusion after liver resection and partial liver transplantation. Transpl Int 2005; 17: 862-71.

45. Hemming AW, Reed Al, Langham MR, Fujita S, van der Werf WJ, Howard RJ. Hepatic vein reconstruction for resection of hepatic tumors. Ann Surg 2002; 235: 850-8.

46. Ferri M, Gabriel S, Gavelli A, Franconeri P, Huguet C. Bacterial translocation during portal clamping for liver resection. A clinical study. Arch Surg 1997; 132: 162-5.

47. Poon RT, Fan ST. Hepatectomy for hepatocellular carcinoma: patient selection and postoperative outcome. Liver Transpl 2004; 10: S39-45.

48. Karoui M, Penna C, Amin-Hashem M, Mitry E, Benoist S, Franc B, et al. Influence of preoperative chemotherapy on the risk of major hepatectomy for colorectal liver metastases. Ann Surg 2006; 243: 1-7.

49. Farges O, Malassagne B, Flejou JF, Balzan S, Sauvanet A, Belghiti J. Risk of major liver resection in patients with underlying chronic liver disease: a reappraisal. Ann Surg 1999; 229: 210-5.

50. Little SA, Jarnagin WR, DeMatteo RP, Blumgart LH, Fong Y. Diabetes is associated with increased perioperative mortality but equivalent long-term outcome after hepatic resection for colorectal cancer. J Gastrointest Surg 2002; 6: 88-94.

51. Yigitler C, Farges O, Kianmanesh R, Regimbeau JM, Abdalla EK, Belghiti J. The small remnant liver after major liver resection: how common and how relevant? Liver Transpl 2003; 9: S18-25.

52. Breitenstein S, Apestegui C, Petrowsky H, Clavien PA. "State of the art" in liver resection and living donor liver transplantation: a worldwide survey of 100 liver centers. World J Surg 2009; 33: 797803.

53. Fan ST. Methods and related drawbacks in the estimation of surgical risks in cirrhotic patients undergoing hepatectomy. Hepatogastroenterology 2002; 49: 17-20.

54. Narita M, Oussoultzoglou E, Fuchshuber P, Pessaux P, Chenard MP, Rosso E, et al. What Is a Safe Future Liver Remnant Size in Patients Undergoing Major Hepatectomy for Colorectal Liver Metastases and Treated by Intensive Preoperative Chemotherapy? Ann Surg Oncol 2012; 19: 2526-38.

55. Kooby DA, Stockman J, Ben-Porat L, Gonen M, Jarnagin WR, Dematteo RP, et al. Influence of transfusions on perioperative and long-term outcome in patients following hepatic resection for colorectal metastases. Ann Surg 2003; 237: 860-9.

56. Luyer MD, Buurman WA, Hadfoune M, Jacobs JA, Konstantinov SR, Dejong CH, et al. Pretreatment with high-fat enteral nutrition reduces endotoxin and tumor necrosis factor-alpha and preserves gut barrier function early after hemorrhagic shock. Shock 2004; 21: 65-71.

57. Yokoyama Y, Schwacha MG, Samy TS, Bland KI, Chaudry IH. Gender dimorphism in immune responses following trauma and hemorrhage. Immunol Res 2002; 26: 63-76.

58. Koperna T, Kisser M, Schulz F. Hepatic resection in the elderly. World J Surg 1998; 22: 406-12. 
59. Suttner SW, Surder C, Lang K, Piper SN, Kumle B, Boldt J. Does age affect liver function and the hepatic acute phase response after major abdominal surgery? Intensive Care Med 2001; 27: 17629.

60. lakova P, Awad SS, Timchenko NA. Aging reduces proliferative capacities of liver by switching pathways of C/EBPalpha growth arrest. Cell 2003; 113: 495-506.

61. Kooby DA, Fong Y, Suriawinata A, Gonen M, Allen PJ, Klimstra DS, et al. Impact of steatosis on perioperative outcome following hepatic resection. J Gastrointest Surg 2003; 7: 1034-44.

62. Mathur AK, Ghaferi AA, Osborne NH, PawlikTM, Campbell DA, Englesbe MJ, et al. Body mass index and adverse perioperative outcomes following hepatic resection. J Gastrointest Surg 2010; 14: 1285-91.

63. Reddy SK, Marsh JW, Varley PR, Mock BK, Chopra KB, Geller DA, et al. Underlying steatohepatitis, but not simple hepatic steatosis, increases morbidity after liver resection: A case-control study. Hepatology 2012; 56: 2221-30.

64. Seifalian AM, Piasecki C, Agarwal A, Davidson BR. The effect of graded steatosis on flow in the hepatic parenchymal microcirculation. Transplantation 1999; 68: 780-4.

65. Serafin A, Rosello-Catafau J, Prats N, Xaus C, Gelpi E, Peralta C. Ischemic preconditioning increases the tolerance of Fatty liver to hepatic ischemia-reperfusion injury in the rat. Am J Pathol 2002; 161: 587-601.

66. Selzner M, Clavien PA. Failure of regeneration of the steatotic rat liver: disruption at two different levels in the regeneration pathway. Hepatology 2000; 31: 35-42.

67. Marcos A, Fisher RA, Ham JM, Shiffman ML, Sanyal AJ, Luketic VA, et al. Liver regeneration and function in donor and recipient after right lobe adult to adult living donor liver transplantation. Transplantation 2000; 69: 1375-9.

68. Bruix J, Castells A, Bosch J, Feu F, Fuster J, Garcia-Pagan JC, et al. Surgical resection of hepatocellular carcinoma in cirrhotic patients: prognostic value of preoperative portal pressure. Gastroenterology 1996; 111: 1018-22.

69. Lau W, Leung K, Leung TW, Liew CT, Chan MS, Yu SC, et al. A logical approach to hepatocellular carcinoma presenting with jaundice. Ann Surg 1997; 225: 281-5.

70. Hemming AW, Scudamore CH, Shackleton CR, Pudek M, Erb SR. Indocyanine green clearance as a predictor of successful hepatic resection in cirrhotic patients. Am J Surg 1992; 163: 515-8.

71. Nordlinger B, Sorbye H, Glimelius B, Poston GJ, Schlag PM, Rougier P, et al. Perioperative chemotherapy with FOLFOX4 and surgery versus surgery alone for resectable liver metastases from colorectal cancer (EORTC Intergroup trial 40983): a randomised controlled trial. Lancet 2008; 371: 1007-16.

72. Nakano H, Oussoultzoglou E, Rosso E, Casnedi S, Chenard-Neu MP, Dufour P, et al. Sinusoidal Injury Increases Morbidity After Major Hepatectomy in Patients With Colorectal Liver Metastases Receiving Preoperative Chemotherapy. Ann Surg 2008; 247: 118-124.

73. Soubrane O, Brouquet A, Zalinski S, Terris B, Brezault C, Mallet V, et al. Predicting high grade lesions of sinusoidal obstruction syndrome related to oxaliplatin-based chemotherapy for colorectal liver metastases: correlation with post-hepatectomy outcome. Ann Surg 2010; 251: 454-60.

74. Rubbia-Brandt L, Audard V, Sartoretti P, Roth AD, Brezault C, Le Charpentier M, et al. Severe hepatic sinusoidal obstruction associated with oxaliplatin-based chemotherapy in patients with metastatic colorectal cancer. Ann Oncol 2004; 15: 460-6.

75. Rubbia-Brandt L, Lauwers GY, Wang H, Majno PE, Tanabe K, Zhu AX, et al. Sinusoidal obstruction syndrome and nodular regenerative hyperplasia are frequent oxaliplatin-associated liver lesions 
and partially prevented by bevacizumab in patients with hepatic colorectal metastasis. Histopathology 2010; 56: 430-9.

76. Schiffer E, Frossard JL, Rubbia-Brandt L, Mentha G, Pastor CM. Hepatic regeneration is decreased in a rat model of sinusoidal obstruction syndrome. J Surg Oncol 2009; 99: 439-46.

77. Narita M, Oussoultzoglou E, Chenard MP, Rosso E, Casnedi S, Pessaux P, et al. Sinusoidal obstruction syndrome compromises liver regeneration in patients undergoing two-stage hepatectomy with portal vein embolization. Surg Today 2011; 41: 7-17.

78. Fong Y, Bentrem DJ. CASH (Chemotherapy-Associated Steatohepatitis) costs. Ann Surg 2006; 243: 8-9.

79. Mullin EJ, Metcalfe MS, Maddern GJ. How much liver resection is too much? Am J Surg 2005; 190: 87-97.

80. Zimmermann H, Reichen J. Hepatectomy: preoperative analysis of hepatic function and postoperative liver failure. Dig Surg 1998; 15: 1-11.

81. Lamade W, Glombitza G, Fischer L, Chiu P, Cardenas CE, Sr., Thorn M, et al. The impact of 3-dimensional reconstructions on operation planning in liver surgery. Arch Surg 2000; 135: 1256-61.

82. Lang H, Radtke A, Liu C, Fruhauf NR, Peitgen HO, Broelsch CE. Extended left hepatectomy--modified operation planning based on three-dimensional visualization of liver anatomy. Langenbecks Arch Surg 2004; 389: 306-10.

83. Wigmore SJ, Redhead DN, Yan XJ, Casey J, Madhavan K, Dejong CH, et al. Virtual hepatic resection using three-dimensional reconstruction of helical computed tomography angioportograms. Ann Surg 2001; 233: 221-6.

84. Schwenzer NF, Springer F, Schraml C, Stefan N, Machann J, Schick F. Non-invasive assessment and quantification of liver steatosis by ultrasound, computed tomography and magnetic resonance. $J$ Hepatol 2009; 51: 433-45.

85. Overman MJ, Maru DM, Charnsangavej C, Loyer EM, Wang H, Pathak P, et al. Oxaliplatin-mediated increase in spleen size as a biomarker for the development of hepatic sinusoidal injury. $J$ Clin Oncol 2010; 28: 2549-55.

86. Dello SA, van Dam RM, Slangen JJ, van de Poll MC, Bemelmans MH, Greve JW, et al. Liver volumetry plug and play: do it yourself with ImageJ. World J Surg 2007; 31: 2215-21.

87. van der Vorst JR, van Dam RM, van Stiphout RS, van den Broek MA, Hollander IH, Kessels AG, et al. Virtual liver resection and volumetric analysis of the future liver remnant using open source image processing software. World J Surg 2010; 34: 2426-33.

88. Vauthey JN, Abdalla EK, Doherty DA, Gertsch P, Fenstermacher MJ, Loyer EM, et al. Body surface area and body weight predict total liver volume in Western adults. Liver Transpl 2002; 8: 233-40.

89. Lau H, Man K, Fan ST, Yu WC, Lo CM, Wong J. Evaluation of preoperative hepatic function in patients with hepatocellular carcinoma undergoing hepatectomy. Br J Surg 1997; 84: 1255-9.

90. Redaelli CA, Dufour JF, Wagner M, Schilling M, Husler J, Krahenbuhl L, et al. Preoperative galactose elimination capacity predicts complications and survival after hepatic resection. Ann Surg 2002; 235: 77-85.

91. Ercolani G, Grazi GL, Calliva R, Pierangeli F, Cescon M, Cavallari A, et al. The lidocaine (MEGX) test as an index of hepatic function: its clinical usefulness in liver surgery. Surgery 2000; 127: 464-71.

92. Hepner GW, Vesell ES. Quantitative assessment of hepatic function by breath analysis after oral administration of (14C)aminopyrine. Ann Intern Med 1975; 83: 632-8.

93. Gill RA, Goodman MW, Golfus GR, Onstad GR, Bubrick MP. Aminopyrine breath test predicts surgical risk for patients with liver disease. Ann Surg 1983; 198: 701-4.

94. Child CG, Turcotte JG. Surgery and portal hypertension. Major Prob/ Clin Surg 1964; 1: 1-85. 
95. Malinchoc M, Kamath PS, Gordon FD, Peine CJ, Rank J, ter Borg PC. A model to predict poor survival in patients undergoing transjugular intrahepatic portosystemic shunts. Hepatology 2000; 31: 864-71.

96. Llovet JM, Bru C, Bruix J. Prognosis of hepatocellular carcinoma: the BCLC staging classification. Semin Liver Dis 1999; 19: 329-38.

97. Cucchetti A, Ercolani G, Vivarelli M, Cescon M, Ravaioli M, La Barba G, et al. Impact of model for end-stage liver disease (MELD) score on prognosis after hepatectomy for hepatocellular carcinoma on cirrhosis. Liver Transpl 2006; 12: 966-71.

98. Madoff DC, Abdalla EK, Vauthey JN. Portal vein embolization in preparation for major hepatic resection: evolution of a new standard of care. J Vasc Interv Radiol 2005; 16: 779-90.

99. Azoulay D, Castaing D, Smail A, Adam R, Cailliez V, Laurent A, et al. Resection of nonresectable liver metastases from colorectal cancer after percutaneous portal vein embolization. Ann Surg 2000; 231: 480-6.

100. Imamura H, Shimada R, Kubota M, Matsuyama Y, Nakayama A, Miyagawa S, et al. Preoperative portal vein embolization: an audit of 84 patients. Hepatology 1999; 29: 1099-105.

101. Kokudo N, Tada K, Seki M, Ohta H, Azekura K, Ueno M, et al. Proliferative activity of intrahepatic colorectal metastases after preoperative hemihepatic portal vein embolization. Hepatology 2001; 34: 267-72.

102. de Santibanes E, Alvarez FA, Ardiles V. How to avoid postoperative liver failure: a novel method. World J Surg 2012; 36: 125-8.

103. Stutchfield BM, Rashid S, Forbes SJ, Wigmore SJ. Practical barriers to delivering autologous bone marrow stem cell therapy as an adjunct to liver resection. Stem Cells Dev 2010; 19: 155-62.

104. Adam R, Laurent A, Azoulay D, Castaing D, Bismuth H. Two-stage hepatectomy: A planned strategy to treat irresectable liver tumors. Ann Surg 2000; 232: 777-85.

105. Jaeck D, Oussoultzoglou E, Rosso E, Greget M, Weber JC, Bachellier P. A two-stage hepatectomy procedure combined with portal vein embolization to achieve curative resection for initially unresectable multiple and bilobar colorectal liver metastases. Ann Surg 2004; 240: 1037-49.

106. Clavien PA, Petrowsky H, DeOliveira ML, Graf R. Strategies for safer liver surgery and partial liver transplantation. N Engl J Med 2007; 356: 1545-59.

107. Melendez JA, Arslan V, Fischer ME, Wuest D, Jarnagin WR, Fong Y, et al. Perioperative outcomes of major hepatic resections under low central venous pressure anesthesia: blood loss, blood transfusion, and the risk of postoperative renal dysfunction. J Am Coll Surg 1998; 187: 620-5.

108. Jones RM, Moulton CE, Hardy KJ. Central venous pressure and its effect on blood loss during liver resection. Br J Surg 1998; 85: 1058-60.

109. Dixon E, Vollmer CM, Jr., Bathe OF, Sutherland F. Vascular occlusion to decrease blood loss during hepatic resection. Am J Surg 2005; 190: 75-86.

110. Belghiti J, Noun R, Zante E, Ballet T, Sauvanet A. Portal triad clamping or hepatic vascular exclusion for major liver resection. A controlled study. Ann Surg 1996; 224: 155-61.

111. Belghiti J, Noun R, Malafosse R, Jagot P, Sauvanet A, Pierangeli F, et al. Continuous versus intermittent portal triad clamping for liver resection: a controlled study. Ann Surg 1999; 229: 369-75.

112. Fernandez L, Carrasco-Chaumel E, Serafin A, Xaus C, Grande L, Rimola A, et al. Is ischemic preconditioning a useful strategy in steatotic liver transplantation? Am J Transplant 2004; 4: 888-99.

113. Clavien PA, Selzner M, Rudiger HA, Graf R, Kadry Z, Rousson V, et al. A prospective randomized study in 100 consecutive patients undergoing major liver resection with versus without ischemic preconditioning. Ann Surg 2003; 238: 843-50. 
114. Kanoria S, Jalan R, Davies NA, Seifalian AM, Williams R, Davidson BR. Remote ischaemic preconditioning of the hind limb reduces experimental liver warm ischaemia-reperfusion injury. Br J Surg 2006; 93: 762-8.

115. Koti RS, Yang W, Dashwood MR, Davidson BR, Seifalian AM. Effect of ischemic preconditioning on hepatic microcirculation and function in a rat model of ischemia reperfusion injury. Liver Transpl 2002; 8: 1182-91.

116. Petrowsky H, McCormack L, Trujillo M, Selzner M, Jochum W, Clavien PA. A prospective, randomized, controlled trial comparing intermittent portal triad clamping versus ischemic preconditioning with continuous clamping for major liver resection. Ann Surg 2006; 244: 921-8.

117. Hwang S, Lee SG, Jang SJ, Cho SH, Kim KH, Ahn CS, et al. The effect of donor weight reduction on hepatic steatosis for living donor liver transplantation. Liver Transp/ 2004; 10: 721-5.

118. Nakamuta M, Morizono S, Soejima Y, Yoshizumi T, Aishima S, Takasugi S, et al. Short-term intensive treatment for donors with hepatic steatosis in living-donor liver transplantation. Transplantation 2005; 80: 608-12.

119. Zein CO, Yerian LM, Gogate P, Lopez R, Kirwan JP, Feldstein AE, et al. Pentoxifylline improves nonalcoholic steatohepatitis: a randomized placebo-controlled trial. Hepatology 2011; 54: 1610-9.

120. Lautz HU, Selberg O, Korber J, Burger M, Muller MJ. Protein-calorie malnutrition in liver cirrhosis. Clin Investig 1992; 70: 478-86.

121. Fan ST, Lo CM, Lai EC, Chu KM, Liu CL, Wong J. Perioperative nutritional support in patients undergoing hepatectomy for hepatocellular carcinoma. N Engl J Med 1994; 331: 1547-52.

122. Robinson SM, Wilson CH, Burt AD, Manas DM, White SA. Chemotherapy-Associated Liver Injury in Patients with Colorectal Liver Metastases: A Systematic Review and Meta-analysis. Ann Surg Oncol 2012; 19: 4287-99.

123. Brouquet A, Benoist S, Julie C, Penna C, Beauchet A, Rougier P, et al. Risk factors for chemotherapy-associated liver injuries: A multivariate analysis of a group of 146 patients with colorectal metastases. Surgery 2009; 145: 362-71.

124. Mahfud M, Breitenstein S, El-Badry AM, Puhan M, Rickenbacher A, Samaras $P$, et al. Impact of preoperative bevacizumab on complications after resection of colorectal liver metastases: casematched control study. World J Surg 2010; 34: 92-100.

125. Agostini J, Benoist S, Seman M, Julie C, Imbeaud S, Letourneur F, et al. Identification of molecular pathways involved in oxaliplatin-associated sinusoidal dilatation. J Hepatol 2012; 56: 869-76.

126. Kimura F, Shimizu H, Yoshidome H, Ohtsuka M, Kato A, Yoshitomi H, et al. Circulating cytokines, chemokines, and stress hormones are increased in patients with organ dysfunction following liver resection. J Surg Res 2006; 133: 102-12.

127. Thasler WE, Bein T, Jauch KW. Perioperative effects of hepatic resection surgery on hemodynamics, pulmonary fluid balance, and indocyanine green clearance. Langenbecks Arch Surg 2002; 387: 271-5.

128. Slankamenac K, Breitenstein S, Held U, Beck-Schimmer B, Puhan MA, Clavien PA. Development and validation of a prediction score for postoperative acute renal failure following liver resection. Ann Surg 2009; 250: 720-8.

129. Matsumata T, Taketomi A, Fujiwara Y, Shimada M, Takenaka K, Sugimachi K. Renal function after elective hepatic resection. Hepatogastroenterology 1996; 43: 602-7.

130. Olde Damink SW, Jalan R, Deutz NE, Redhead DN, Dejong CH, Hynd P, et al. The kidney plays a major role in the hyperammonemia seen after simulated or actual Gl bleeding in patients with cirrhosis. Hepatology 2003; 37: 1277-85. 
131. Ferenci P, Lockwood A, Mullen K, Tarter R, Weissenborn K, Blei AT. Hepatic encephalopathy-definition, nomenclature, diagnosis, and quantification: final report of the working party at the 11 th World Congresses of Gastroenterology, Vienna, 1998. Hepatology 2002; 35: 716-21.

132. Shawcross D, Jalan R. The pathophysiologic basis of hepatic encephalopathy: central role for ammonia and inflammation. Cell Mol Life Sci 2005; 62: 2295-304.

133. Jalan R, Olde Damink SW, Hayes PC, Deutz NE, Lee A. Pathogenesis of intracranial hypertension in acute liver failure: inflammation, ammonia and cerebral blood flow. J Hepatol 2004; 41: 613-20.

134. Rivers E, Nguyen B, Havstad S, Ressler J, Muzzin A, Knoblich B, et al. Early goal-directed therapy in the treatment of severe sepsis and septic shock. N Engl J Med 2001; 345: 1368-77.

135. Jalan R. Pathophysiological basis of therapy of raised intracranial pressure in acute liver failure. Neurochem Int 2005; 47: 78-83.

136. Wu CC, Yeh DC, Lin MC, Liu TJ, P'Eng F K. Prospective randomized trial of systemic antibiotics in patients undergoing liver resection. Br J Surg 1998; 85: 489-93.

137. Togo S, Tanaka K, Matsuo K, Nagano Y, Ueda M, Morioka D, et al. Duration of antimicrobial prophylaxis in patients undergoing hepatectomy: a prospective randomized controlled trial using flomoxef. J Antimicrob Chemother 2007; 59: 964-70.

138. Rolando N, Gimson A, Wade J, Philpott-Howard J, Casewell M, Williams R. Prospective controlled trial of selective parenteral and enteral antimicrobial regimen in fulminant liver failure. Hepatology 1993; 17: 196-201.

139. Ezzat TM, Dhar DK, Newsome PN, Malago M, Olde Damink SW. Use of hepatocyte and stem cells for treatment of post-resectional liver failure: are we there yet? Liver Int 2011; 31: 773-84.

140. Stutchfield BM, Simpson K, Wigmore SJ. Systematic review and meta-analysis of survival following extracorporeal liver support. Br J Surg 2011; 98: 623-31.

141. Onodera K, Sakata H, Yonekawa M, Kawamura A. Artificial liver support at present and in the future. J Artif Organs 2006; 9: 17-28.

142. Liu J, Kjaergard LL, Als-Nielsen B, Gluud C. Artificial and bioartificial support systems for liver failure: a Cochrane Hepato-Biliary Group Protocol. Liver 2002; 22: 433-8.

143. Sen S, Williams R, Jalan R. Emerging indications for albumin dialysis. Am J Gastroenterol 2005; 100: 468-75.

144. Krisper P, Stauber RE. Technology insight: artificial extracorporeal liver support--how does Prometheus compare with MARS? Nat Clin Pract Nephrol 2007; 3: 267-76.

145. Heemann U, Treichel U, Loock J, Philipp T, Gerken G, Malago M, et al. Albumin dialysis in cirrhosis with superimposed acute liver injury: a prospective, controlled study. Hepatology 2002; 36: 94958.

146. Rittler P, Ketscher C, Inthorn D, Jauch KW, Hartl WH. Use of the molecular adsorbent recycling system in the treatment of postoperative hepatic failure and septic multiple organ dysfunction-preliminary results. Liver Int 2004; 24: 136-41.

147. van de Kerkhove MP, de Jong KP, Rijken AM, de Pont AC, van Gulik TM. MARS treatment in posthepatectomy liver failure. Liver Int 2003; 23 Suppl 3: 44-51.

148. Kellersmann R, Gassel HJ, Buhler C, Thiede A, Timmermann W. Application of Molecular Adsorbent Recirculating System in patients with severe liver failure after hepatic resection or transplantation: initial single-centre experiences. Liver 2002; 22 Suppl 2: 56-8.

149. Krisper P, Haditsch B, Stauber R, Jung A, Stadlbauer V, Trauner M, et al. In vivo quantification of liver dialysis: comparison of albumin dialysis and fractionated plasma separation. $J$ Hepatol 2005; 43: 451-7. 
150. Demetriou AA, Brown RS, Jr., Busuttil RW, Fair J, McGuire BM, Rosenthal P, et al. Prospective, randomized, multicenter, controlled trial of a bioartificial liver in treating acute liver failure. Ann Surg 2004; 239: 660-7.

151. Ringe B, Lubbe N, Kuse E, Frei U, Pichlmayr R. Total hepatectomy and liver transplantation as two-stage procedure. Ann Surg 1993; 218: 3-9.

152. Cucchetti A, Ercolani G, Cescon M, Ravaioli M, Zanello M, Del Gaudio M, et al. Recovery from liver failure after hepatectomy for hepatocellular carcinoma in cirrhosis: meaning of the model for end-stage liver disease. J Am Coll Surg 2006; 203: 670-6.

153. Otsuka Y, Duffy JP, Saab S, Farmer DG, Ghobrial RM, Hiatt JR, et al. Postresection hepatic failure: Successful treatment with liver transplantation. Liver Transp/ 2007; 13: 672-9.

154. Lock JF, Reinhold T, Malinowski M, Pratschke J, Neuhaus P, Stockmann M. The costs of postoperative liver failure and the economic impact of liver function capacity after extended liver resectiona single-center experience. Langenbecks Arch Surg 2009; 394: 1047-56. 



\section{Part I}

Assessing outcomes of liver surgery:

critical appraisal of clinical endpoints 



\section{Chapter 2}

\section{Feasibility of randomized controlled trials in liver surgery using surgery-related mortality or morbidity as endpoint}

Published as:

van den Broek MA, van Dam RM, Malagó M, Dejong CH, van Breukelen GJ, Olde Damink SW. Feasibility of randomized controlled trials in liver surgery using surgeryrelated mortality or morbidity as endpoint. Br J Surg 2009; 96: 1005-1014. 


\section{ABSTRACT}

\section{Background}

There is a shortage of randomized controlled trials (RCTs) on which to base guidelines in liver surgery. The feasibility of conducting an adequately powered RCT in liver surgery using the dichotomous endpoints surgery-related mortality or morbidity was studied.

\section{Methods}

Articles published between January 2002 and November 2007 with mortality or morbidity after liver surgery as primary endpoint were retrieved, based on which mean incidence rates were calculated. Sample size calculations for an RCT aiming to show a relative reduction of these endpoints by 33,50 or 66 per cent were performed.

\section{Results}

The mean operative mortality rate was 1.0 per cent and the total morbidity rate 28.9 per cent; mean rates of bile leakage and post-resectional liver failure were 4.4 and 2.6 per cent, respectively. The smallest numbers of patients needed in each arm of an RCT aiming to show a one-third relative reduction were 15614 for operative mortality, 412 for total morbidity, 3446 for bile leakage and 5924 for post-resectional liver failure.

\section{Conclusion}

The feasibility of conducting an adequately powered RCT in liver surgery using dichotomous outcomes such as mortality or specific complications seems low. Conclusions of underpowered RCTs should be interpreted with caution. Implementation of a liver surgery specific composite endpoint may be a useful and clinically relevant solution to pursue. 


\section{INTRODUCTION}

The application of evidence-based medicine aims to improve rational and consistent decision making. It combines the best available external clinical evidence with individual clinical expertise ${ }^{1}$. Evidence-based guidelines should preferably be based on the results of randomized controlled trials (RCTs) that test the effectiveness of a new treatment against the standard treatment with respect to a predetermined, clinically relevant endpoint. However, RCTs are scarce in liver surgery ${ }^{2}$.

The safety of partial liver resection has improved considerably in the past decade $\mathrm{e}^{3-5}$. As curative oncological resections are being performed for even larger tumours, the minimum remnant liver volume is constantly being re-evaluated. As a result, complications such as bile leakage, intra-abdominal abscess or post-resectional liver failure remain a major threat to the patient. RCTs testing the efficacy of innovative techniques in lowering these complication rates are desirable.

RCTs that use dichotomous endpoints such as surgery-related mortality, total morbidity or specific complications as primary endpoints generate the strongest evidence and are necessary to obtain approval of the US Food and Drug Administration. However, these endpoints might increase the complexity of an RCT because they often necessitate large sample sizes. A state-of-the-art calculation of the smallest number of patients needed to be enrolled is an essential first step in the design of a randomized trial. If too few patients are enrolled, a trial testing a treatment with a clinically important treatment effect may fail to show a statistically significant effect owing to lack of statistical power, whereas inclusion of too many patients may be regarded as unethical, costly and inefficient. Unfortunately, the performance and reporting of sample size calculations is suboptimal in both the surgical and hepatobiliary literature ${ }^{2,6,7}$.

The aim of the present study was to determine the feasibility of conducting an adequately powered RCT in liver surgery using the dichotomous endpoints surgery-related mortality or morbidity. A systematic review was undertaken to estimate the current rates of mortality, total morbidity and specific complications of liver surgery. Based on these rates, the minimum sample size needed to conduct an adequately powered RCT that would be able to show a relative reduction in surgery-related mortality or morbidity by at least one-third was calculated.

\section{METHODS}

\section{Search strategy}

A systematic, computerized literature search (PubMed, the Cochrane Library and Embase) was performed using the key words "hepatectomy OR liver resection" in order 
to identify all studies that described clinical outcomes of liver surgery. The search was conducted to determine the rates of liver surgery-related mortality, total morbidity and specific complications in current HPB practice. The search term was limited to articles published in English between January 2002 and November 2007 that included patients older than 19 years of age. Article reference lists were cross-checked for additional useful papers to increase the sensitivity of the search. The outcome measures were 30-day, operative and/or in-hospital mortality and total morbidity, procedure specific morbidity (bile leakage and post-resectional liver failure) and other types of morbidity, depending on the outcome measures reported.

\section{Study eligibility}

Studies were eligible if they were consecutive case series, came from high-volume centres (carrying out more than 15 resections per year ${ }^{8}$ ), included a minimum of 50 patients, and reported on a case mix of patients and resections. Patients undergoing repeat resections were also included. Each article had to describe the study interval, study characteristics (patient number and presence of exclusion criteria), operative data (total number of resections), one mortality index (30-day, operative or in-hospital mortality), total morbidity and at least one procedure specific complication. Procedure specific complications were defined as bile leakage and post-resectional liver failure. When the same institute reported two studies using data from overlapping periods, the one of better quality was selected based on a combination of the criteria reported by Martin and colleagues and Moher and co-workers ${ }^{9,10}$. The methodological quality of these papers was assessed by a sum score. An RCT scored 5 points, a prospective trial 3 points, and all other positive quality items on the checklist scored 1 point, leading to a maximum quality score of 19 points (Appendix 1).

Studies were excluded if they (1) reported on other outcomes or were irrelevant to the subject under study (for example animal studies or studies on liver transplantation), (2) used aggregated data from nation-wide data sets, (3) only described a specific patient group, (4) were reviews, (5) lacked detailed data on either mortality or total or procedure specific morbidity.

\section{Selection and data extraction}

Two authors independently performed the computerized search and selected abstracts that met the predetermined eligibility terms for full-text examination. Thereafter, two authors judged the suitability of these studies for inclusion in the final analysis by using a standardized checklist (Appendix 1, items marked with an asterisk). Data of the selected studies were extracted by one author and independently reviewed by two other authors. Disagreements were resolved by discussion. 
The following data were recorded systematically: first author, year of publication, institute name and location, continent of origin, study design, study interval, number of patients, sex, age, number, type and indication for liver resection, presence of data on and definition of procedure specific complications (bile leakage and post-resectional liver failure), and duration of follow-up. The incidence of the following post-resectional complications was recorded, if explicitly mentioned: 30-day, operative or in-hospital mortality, total morbidity, post-resectional liver failure, bile leakage, ascites, sepsis, intra-abdominal abscess, ileus, pleural effusion, pneumonia, pneumothorax, urinary tract infection, renal insufficiency, cardiac arrhythmia, intra-abdominal hemorrhage, wound infection and dehiscence.

\section{Definition of outcome measures}

Resections were regarded as major when three or more segments were involved and minor when one or two segments were involved or a non-anatomical resection was performed ${ }^{11}$.

Mortality was classified into 30-day, operative and in-hospital mortality, dependent on the mortality index used in the article. Total morbidity included all complications that prolonged the patients' hospital stay or had a life-threatening impact, dependent on the post-resectional complications accounting for total morbidity per article. Self-limiting post-operative events were regarded as minor complications, and were grouped as grade 1 or 2 according to the classification index of Dindo and colleagues ${ }^{12}$. Major complications comprised those requiring surgical, endoscopic or radiological intervention, or resulting in multi-organ failure or death (Dindo grades 3 to 5).

\section{Mortality and morbidity rates after liver resection}

The rates of surgery-related mortality, total morbidity and specific complications in current HPB practice were calculated by means of a mixed effects logistic regression meta-analysis. Mean rates and between-study variance (heterogeneity) of the rates were determined for each complication. Only complications that were documented by at least half of the studies selected for final analysis were included in the mixed effects logistic regression meta-analysis. For surgery-related mortality and total morbidity, these meta-analyses were repeated with design (prospective versus retrospective) as predictor of the mean incidence rate.

\section{Sample size calculation}

Sample size was calculated based on these mean rates with the assumption that an (hypothetical) RCT should be able to detect a clinically meaningful, relative reduction in surgery-related mortality, total morbidity or specific complications by one-third, onehalf or two-thirds. The null-hypothesis was that there was no difference between the 
intervention and standard treatment in the incidence rate of the primary endpoint. All sample size calculations assumed two-sided testing.

The sample size of each arm was calculated using the equation designed for two proportions (as described by Kirkwood and colleagues ${ }^{13}$ and illustrated in Appendix 2); a was set at 0.05 to control for type I error (false-positive result) and $\beta$ at 0.10 to control for type II error (false-negative result). Only one primary endpoint was chosen for each hypothetical RCT, as testing for multiple outcome parameters would require correction for multiple comparisons and thereby increase the sample size.

\section{Effect of change in study design on sample size}

The calculated sample sizes were based on a single-centre trial with no dropouts and a level of significance $\alpha=0.05$ and power $1-\beta=0.90$. Changes in these assumptions result in changes in the sample size.

\section{Random dropout}

The expected loss to follow-up owing to random dropout from the intervention and control groups was taken into account by increasing the sample size with the correction factor $=100 /(100-\mathrm{K})$ where $\mathrm{K}$ represents the percentage dropout. This is a remedy against loss of power owing to random dropout only, and is not a solution to bias because of selective dropout, which requires advanced statistics ${ }^{14}$.

\section{Multicentre trial}

A larger patient population can be obtained by running a multicentre trial. However, centre and treatment-by-centre effects on the outcome increase the sample size needed for a given effect size and power. Without treatment-by-centre interaction, an increase of the sample size of each arm of an RCT with $C$, where $C$ denotes the number of centres, is sufficient. With interaction, however, a larger sample size is needed and its calculation depends on whether centres are treated as fixed or random, and on whether the feasible sample size varies strongly between centres ${ }^{15,16}$.

\section{Change in $A$ and $B$ terms}

$A$ and $B$ terms ( $\alpha$ and $\beta$ respectively) are set at the beginning of a study, but their values are open to discussion. An a of 0.01 instead of 0.05 may be considered in case of multiple outcomes (Bonferroni correction for multiple comparisons). $A \beta$ of 0.20 instead of 0.10 implies a power of 80 per cent, which is often used in planning studies.

\section{Statistical analysis}

Statistical analysis of study characteristics was performed using SPSS version 12.0.1 for Windows (SPSS Inc., Chicago, IL, USA). The mixed effects logistic regression meta-analysis 
was performed using second order penalized quasi-likelihood estimation with MLwiN 1.100007 (Centre for Multilevel Modelling, Bristol, UK) ${ }^{17}$. This resulted in a mean incidence rate with 95 per cent interval for individual studies for each complication. Essentially, the $\mu$ and $\sigma^{2}$ as estimated by mixed effects logistic regression are the between-study mean and variance of the incidence on the logodds scale. Mean on the percentage scale was obtained by taking $\mu$ and then applying the inverse logodds transformation. The 95 per cent interval was obtained by taking $\mu \pm 2 \sigma$ and then applying the inverse logodds transformation to the interval boundaries multiplying by 100 per cent. Heterogeneity (between-study variance) was tested using a chi-square test of $\sigma^{2}$ (ratio of $\left[\sigma^{2} / \mathrm{SE}\right]^{2}$ ) with a cutoff value of more than 4 (two-sided testing and $a=0.05$ ). Sample sizes are presented as absolute numbers and represent the minimum number of patients needed in each arm of an RCT.

\section{RESULTS}

\section{Search strategy}

A total of 2666 abstracts that met the search terms "hepatectomy OR liver resection" were retrieved. Of these, 2549 were excluded after abstract review (Figure 2.1). The main reasons for exclusion were irrelevance to the subject under study $(n=926)$, small patient numbers ( $n=519$ case reports and $n=351$ studies with fewer than 50 patients) and reporting on other outcomes $(n=314)$. Some 117 potentially eligible articles underwent full text examination. Nineteen of these contained overlapping data, and the ten articles of inferior quality were excluded (Appendix 3). Additional reasons for exclusion are listed in Figure 2.1. Hand searching of the reference lists identified two additional eligible articles. Finally, 32 studies fulfilled all inclusion criteria and were used in the analysis (Appendix 4) $)^{3,18-48}$.

\section{Study and patient characteristics}

The 32 studies contained a total of 8680 patients who underwent 8824 resections (Table 2.1). The median number of patients per study was 128 (range 53 - 1803). Twenty studies were conducted prospectively, nine of which were RCTs; ten were retrospective and two lacked this information. The methodological quality of these 32 articles is summarized in Appendix 4. Fifteen articles reported data originating from European centres, fourteen from Asian centres and three from other continents (one each from North-America, South-America and Australasia). 
Potentially relevant abstracts identified and screened for retrieval, $n=2666$

- PubMed, $n=2461$

- Embase, $n=171$

- Cochrane, $n=34$

\begin{tabular}{|ll}
\hline Abstracts excluded, $n=2549$ \\
$\cdot$ not relevant* \\
$\cdot$ case report & 926 \\
$\cdot<50$ hepatectomies & 519 \\
$\cdot<15$ hepatectomies/year & 351 \\
$\cdot$ subgroups only & 107 \\
$\cdot$ other outcome & 263 \\
$\cdot$ review & 314 \\
$\cdot$ overlap between databases & 44 \\
$\cdot$ nationwide data & 15 \\
$\cdot$ not in English & 8 \\
\hline
\end{tabular}

Articles retrieved for more detailed evaluation, $n=117$

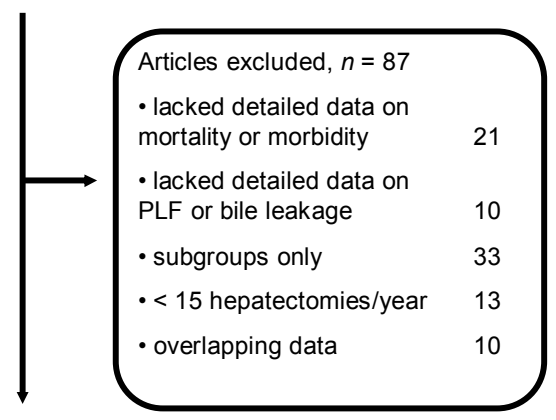

Potentially appropriate articles to be

included in the analysis, $n=30$

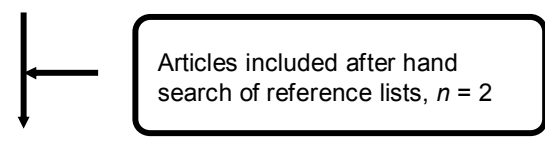

Articles included in final analysis,

$n=32$

Figure 2.1 Selection of studies eligible for final analysis.

Legend: PLF, post-resectional liver failure; $\mathrm{Hx}$, hepatectomy; * e.g. animal studies, genetic studies, studies focussing on liver transplantation. 
Table 2.1 Characteristics of patients described in the articles eligible for final analysis.

\begin{tabular}{lc}
\hline Characteristics & $\mathbf{N = 8 6 8 0}$ \\
\hline Sex ratio (female : male) & $2: 3$ \\
Age (years) (range)* & $0.2-89.0$ \\
Indication for resection ${ }^{\dagger}$ & \\
$\quad$ Primary hepatic malignancy & $3948(46.6)$ \\
$\quad$ Secondary hepatic malignancy & $3484(41.1)$ \\
$\quad$ Benign hepatic disease & $739(8.7)$ \\
$\quad$ Other & $307(3.6)$ \\
Number of resections & 8824 \\
Type of resection & \\
Major hepatectomy & $3577(47.5)$ \\
$\quad$ Minor hepatectomy & $3960(52.5)$ \\
\hline
\end{tabular}

Numbers in parentheses are percentages; ${ }^{*}$ four studies included liver resections in children $29,37,43,45 ;{ }^{\dagger}$ data missing from three studies ${ }^{20,38,43} ;{ }^{*}$ data from three studies not compatible $20,25,39$.

\section{Mortality and morbidity rates after liver resection}

The distribution of rates of surgery-related mortality and morbidity is shown in Figure 2.2 (and Appendix 4). Twenty-nine articles reported the incidence of bile leakage and 25 articles the rate of post-resectional liver failure; 22 reported both. Nine articles provided

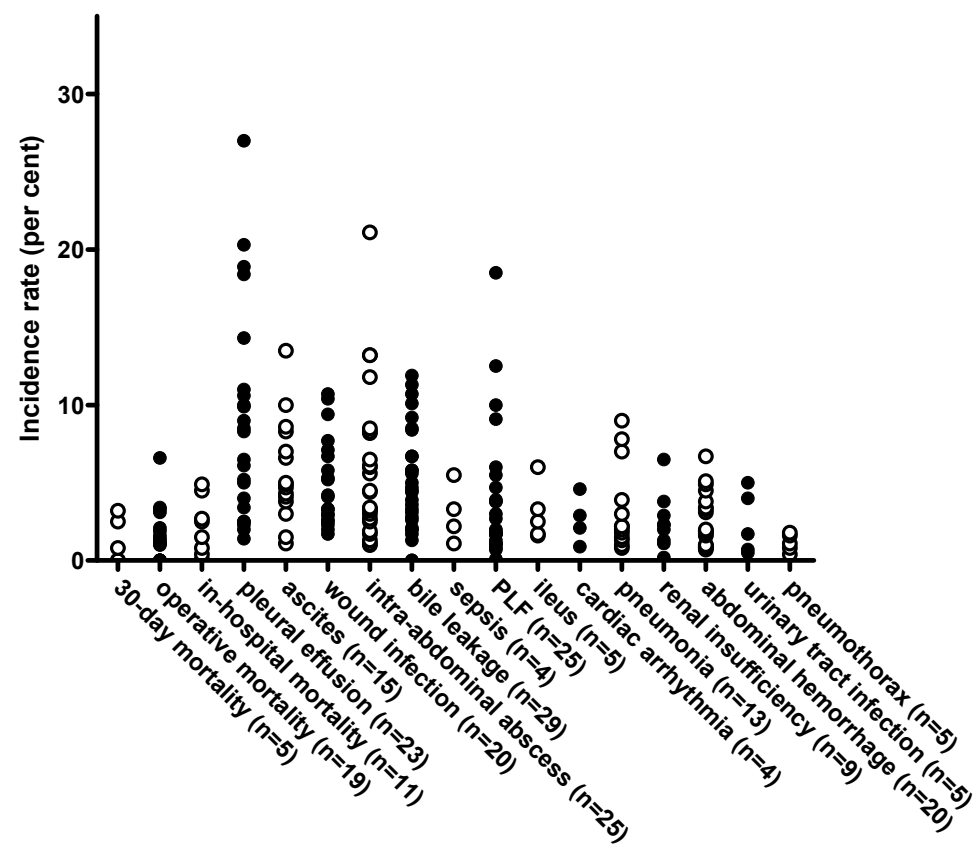

Figure 2.2 Distribution of rates of liver surgery-related mortality and specific complications. Legend: $N$, number of articles reporting on the complication; PLF, post-resectional liver failure. 
a definition of bile leakage and 10 of post-resectional liver failure. Definitions used for these procedure specific complications varied widely. Complications were reviewed during the in-hospital stay in four studies ${ }^{21,34,40,45}$, and during the first 30, 180 and 360 days after operation in three ${ }^{23,24,41}$, one ${ }^{22}$ and one ${ }^{27}$, respectively. This information was lacking in the other 23 articles. Complications were described according to the ClavienDindo classification in four articles only ${ }^{19,23,24,47}$.

The mean rates of surgery-related mortality and specific complications reported by at least 16 articles were low (Table 2.2). Mean operative mortality rate was 1.0 per cent and total morbidity rate $\mathbf{2 8 . 9}$ per cent. Rates were 7.2 per cent for pleural effusion, 4.6 per cent for intra-abdominal abscess and wound infection, 4.4 per cent for bile leakage, 2.6 per cent for post-resectional liver failure and 1.9 per cent for intra-abdominal hemorrhage. The between-study variance (heterogeneity), depicted as $\sigma^{2}$ and 95 per cent intervals in Table 2.2, was significant for all complications except operative mortality and intra-abdominal hemorrhage (which may be due to the small number of studies reporting on these complications).

Mean incidence rates of operative mortality and total morbidity differed between studies with a prospective or retrospective design ( 1.1 versus 0.7 per cent for operative mortality and 27.1 versus 33.9 per cent for total morbidity). However, these differences were non-significant ( $p=0.562$ and $p=0.153$, respectively).

Table 2.2 Mean rates of surgery-related mortality, total morbidity and specific complications after liver resection.

\begin{tabular}{|c|c|c|c|c|c|}
\hline Complication & $N$ & $\mu$ logodds* & $\sigma^{2}$ logodds* & mean rate ${ }^{\dagger}$ & $\begin{array}{c}95 \text { per cent } \\
\text { interval }^{\ddagger}\end{array}$ \\
\hline Operative mortality & 19 & $-4.558(0.316)$ & $1.050(0.576)$ & 1.0 & $0.1-7.5$ \\
\hline Total morbidity & $30^{*}$ & $-0.902(0.105)$ & $0.288(0.085)$ & 28.9 & $12.2-51.9$ \\
\hline \multicolumn{6}{|l|}{ Specific types of morbidity } \\
\hline 1. Pleural effusion & 23 & $-2.551(0.184)$ & $0.632(0.225)$ & 7.2 & $1.6-27.7$ \\
\hline 2. Wound infection and dehiscence & 20 & $-3.032(0.141)$ & $0.242(0.121)$ & 4.6 & $1.8-11.4$ \\
\hline 3. Intra-abdominal abscess & 25 & $-3.028(0.173)$ & $0.569(0.207)$ & 4.6 & $1.1-18.0$ \\
\hline 4. Bile leakage & 29 & $-3.089(0.139)$ & $0.386(0.142)$ & 4.4 & $1.3-13.6$ \\
\hline 5. Post-resectional liver failure & 25 & $-3.635(0.247)$ & $1.152(0.419)$ & 2.6 & $0.3-18.4$ \\
\hline 6. Intra-abdominal hemorrhage & 20 & $-3.951(0.181)$ & $0.328(0.193)$ & 1.9 & $0.6-5.7$ \\
\hline
\end{tabular}

$\mathrm{N}$, number of articles reporting on the complication; ${ }^{*}$ numbers in parentheses are standard error; ${ }^{\dagger} \mathrm{Com}$ puted by inverse logodds transform of $\mu$, giving a proportion, and multiplying by 100 per cent; ${ }^{\ddagger}$ computed by inverse logodds transform of the interval boundaries $\mu \pm 2 \sigma$ and multiplying by 100 per cent; ${ }^{*}$ data on total morbidity, reported in two studies ${ }^{24,47}$, were divided into major and minor morbidity and were therefore not compatible. 


\section{Sample size calculation}

The low rates of surgery-related mortality and morbidity would necessitate a large number of patients in each arm of an adequately powered RCT that aimed to show a relative reduction of the incidence of a complication by one-third, one-half or two-thirds (Table 2.3). For example, if the intention was to show a one-third reduction in the incidence of bile leakage, that is a reduction from the reported 4.4 to 2.9 per cent, 3446 patients would be needed in each arm of a trial to have sufficient power. For an RCT aiming to show a relative reduction in bile leakage by one-half or two-thirds, 1383 or 693 patients, respectively, would be required in each arm.

Because of its much higher rate, use of total morbidity as endpoint would considerably decrease the number of patients needed in each arm of an adequately powered RCT intending to show a reduction by one-third, one-half or two-thirds (Table 2.3). However, total morbidity was ill-defined in most articles.

Table 2.3 Sample size required for each arm of a randomized controlled trial intending to show a relative reduction of surgery-related mortality, total morbidity or specific complications by one-third, one-half or two-thirds.

\begin{tabular}{lccc}
\hline & \multicolumn{3}{c}{ Relative reduction } \\
\cline { 2 - 3 } Complication & $\mathbf{3 3 \%}$ & $\mathbf{5 0 \%}$ & $\mathbf{6 6 \%}$ \\
\hline Operative mortality & 15614 & 6250 & 3127 \\
Total morbidity & 412 & 169 & 86 \\
1. Pleural effusion & 2054 & 826 & 415 \\
2. Wound infection and dehiscence & 3290 & 1320 & 662 \\
3. Intra-abdominal abscess & 3290 & 1320 & 662 \\
4. Bile leakage & 3446 & 1383 & 693 \\
5. Post-resectional liver failure & 5924 & 2374 & 1189 \\
6. Intra-abdominal hemorrhage & 8155 & 3266 & 1635 \\
\hline
\end{tabular}

Assumptions $\alpha=0.05$ and $\beta=0.1$ and two-sided testing.

\section{Effect of change in study design on sample size}

To take into account a random dropout of 10 per cent, the sample size of each arm of an RCT should be increased by 11 per cent. The effects of conducting a multicentre study were less dramatic, at least when assuming an absence of treatment-by-centre interaction. A study with 20 centres would need to include only an extra 20 patients in each arm.

The effects of changes in $A$ and $B$ terms on the sample size of an RCT aiming to show a relative reduction of bile leakage by 33,50 or 66 per cent are illustrated in Figure 2.3. A change in power from 90 per cent $(\beta=0.1)$ to 80 per cent $(\beta=0.2)$ resulted in a decrease of the sample size by approximately 25 per cent (dotted line in Figure 2.3). Changing a from 0.05 to the more stringent 0.01 increased the sample size by 42 per cent (striped line in Figure 2.3). 


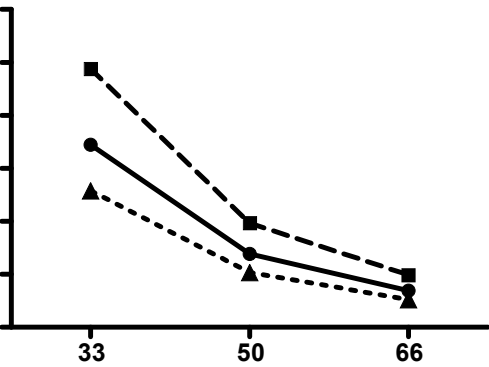

- - alpha 0.01 and beta 0.10

- - alpha 0.05 and beta 0.20

$\rightarrow$ alpha 0.05 and beta 0.10

Relative reduction in rate of bile leakage (\%)

Figure 2.3 The effect of change of a or $\beta$ on the sample size of each arm of a randomized controlled trial that intends to show a relative reduction of bile leakage by one-third, one-half or two-thirds.

\section{DISCUSSION}

The current rates of surgery-related mortality and specific types of morbidity after liver resection were low. Operative mortality rate was only 1.0 per cent and the rates of specific types of morbidity were all below 8 per cent. Consequently, the sample size of an adequately powered RCT using surgery-related mortality or specific types of morbidity as primary endpoint turned out to be extremely large. The smallest numbers of patients to be included in each arm of an RCT that aims to show a relative reduction by one-third were 15614 for operative mortality, 412 for total morbidity, 3446 for bile leakage and 5924 for post-resectional liver failure. Studies using fewer patients can be regarded as underpowered and their results should be interpreted with caution.

This study is limited to short-term surgery-related outcomes. A similar estimation for long-term outcomes after liver surgery requires a different search strategy and was beyond the scope of the present study. Rates of surgery-related mortality, total morbidity and specific types of morbidity after liver resection are gradually decreasing due to better preoperative work-up, operative strategies and perioperative patient care ${ }^{24,32,49}$. The present analysis shows that, because of these declining event rates, it will be increasingly difficult to conduct an adequately powered RCT using a clinical endpoint as primary endpoint. Our Liver Unit is a high-volume centre for liver surgery in the Netherlands and performs approximately 70 liver resections annually. Conduction of a single-centre RCT in our Unit with the intention to show a relative reduction of bile leakage by one-third would require an inclusion period of 99 years. Even in the largest Liver Units in the world (performing about 200 hepatectomies annually ${ }^{3,40,45}$ ), this RCT would take 35 years. Inclusion periods of these lengths are unworkable; moreover, by the end of the trial, any conclusions would no longer be relevant. In addition, the feasibility of a multicentre trial is questionable because only 500 liver resections are performed annually in the Neth- 
erlands and only 7000 in the USA (Netherlands Health Care Inspectorate 2005, www. prismant.nl, and HCUP Nationwide Inpatient Sample 2000, www.hcup-us.ahrq.gov).

Underpowered RCTs are ineffective because the accuracy of their results is uncertain. They might lead to the generation of potentially harmful conclusions that are incorporated in treatment guidelines. The CONSORT statement was developed to overcome this problem ${ }^{10}$. It contains 22 criteria on study design and sample size calculations for RCTs. Although the quality of reported trials improved after the introduction of the CONSORT statement ${ }^{50}$, overall reporting on RCTs in surgical journals is still suboptimal ${ }^{7,9}$.

The minimum number of patients to be included in an RCT might have been overestimated in the present study. Possible confounders are the inclusion of articles of suboptimal quality (for example those lacking definitions of procedure specific complications, those with short follow-up periods, or retrospective case series ${ }^{9,51}$ ), publication bias resulting from selective publication of results from centres of excellence, or the inclusion of studies that assessed a case mix of patients and resections. Although the latter leads to an increased external validity, it may not reflect the actual complication rate in a subgroup of patients.

The overall quality of the selected articles was suboptimal with reporting of both procedure specific complications in only two-thirds and these complications defined in less than half. Furthermore, data collection was retrospective in one-third of studies, and such studies have been shown to be of inferior quality compared with prospective case series ${ }^{9}$. However, no significant differences in rates of operative mortality and total morbidity were found in the present meta-analysis between prospective and retrospective studies.

The feasibility of an RCT could be enhanced by including selective patient groups (such as patients undergoing major liver resection or patients with cirrhosis) or by choosing composite or surrogate endpoints. Some studies have identified major liver resection as an independent predictor of post-resectional morbidity ${ }^{27,45}$. Patients who have undergone major liver resection have higher complication rates and, consequently, smaller sample sizes will be needed. On the other hand, the number of eligible patients will also decrease.

Total morbidity, probably the only outcome resulting in a feasible sample size, was ill-defined and therefore not ideal as a primary endpoint of an RCT. An alternative would be to use a well-defined liver surgery specific composite endpoint, that is a parameter composed of a combination of two or more procedure specific complications that are considered as a single, dichotomous outcome. This increases the event rate and prevents multiplicity, both of which increase the statistical power of an RCT. The authors propose a composite endpoint comprising operative mortality, bile leakage, intra-abdominal abscess, post-resectional liver failure, and intra-abdominal hemorrhage. Such an endpoint is dichotomous and reflects the occurrence of at least one of these components, 
which are all specific to liver surgery and have substantial clinical relevance, reflecting a Clavien-Dindo severity grade of 3 or more ${ }^{12}$. Based on the rates per component (Table 2.2), the incidence of the liver surgery specific composite endpoint can be estimated to lie between 4.6 and 14.5 per cent, depending on the presence of simultaneous complications (the occurrence of two or more of these endpoints in one patient), which is undesirable. A prospective study is necessary to determine the true incidence and validity of this composite endpoint as the individual components might have variable clinical relevance or rates, dependent on the patient cohort under study ${ }^{52}$.

Surrogate endpoints are increasingly being used as they reduce the numbers of patients needed in RCTs by transforming the primary endpoint from a dichotomous into a continuous variable. An essential characteristic of a surrogate endpoint is the ability to reflect the clinical benefit of an intervention accurately. Surrogate endpoints that have been used recently to describe post-resectional outcomes are rather weak, including the biomarkers serum bilirubin and prothrombin index (combined in the so-called 50-50 criteria) for post-resectional liver failure ${ }^{53}$, rise in serum aminotransferase level for ischemia-reperfusion injury ${ }^{49}$ and plasma arterial lactate for mortality ${ }^{21}$. Functional parameters used as surrogate endpoints consist of time to passage of flatus for ileus ${ }^{24}$ or length of hospital stay for total morbidity. However, there is currently no consensus about the validity of these surrogate endpoints, which is a prerequisite for approval of new drugs by the US Food and Drug Administration. Future research should focus on the validation of convincing surrogate endpoints in large prospectively followed cohorts.

The mandatory institution of prospective registries to monitor the safety and therapeutic effectiveness of innovative techniques in HPB surgery that have not been tested by adequately powered RCTs, as suggested by Strasberg and Ludbrook ${ }^{54}$, seems also interesting. These registries could overcome the problem of lack of statistical power of RCTs by compiling high-volume data on mortality or complications after new procedures. The occurrence of unexpected adverse events following the introduction of a procedure could be identified by such a registry despite their low incidence; an example is the increased rate of biliary injury after the introduction of laparoscopic cholecystectomy ${ }^{54}$.

In summary, to achieve the required sample size to conduct an adequately powered $\mathrm{RCT}$ in liver surgery using the dichotomous endpoints mortality, bile leakage or postresectional liver failure, multicentre collaborations or protracted inclusion periods would be required. However, multicentre trials of this size do not seem realistic. Implementation and validation of a liver surgery specific composite endpoint might increase the feasibility of conducting RCTs in liver surgery. 


\section{REFERENCES}

1. Sackett DL, Straus SE, Richardson WS, Rosenberg W, Haynes RB. Evidence-based medicine. How to practice and teach EBM. London: Harcourt Publishers Limited; 2000.

2. Kaido T. Analysis of randomized controlled trials on hepatopancreatic surgery. Dig Dis Sci 2006; 51: 1761-6.

3. Poon RT, Fan ST, Lo CM, Liu CL, Lam CM, Yuen WK, et al. Improving perioperative outcome expands the role of hepatectomy in management of benign and malignant hepatobiliary diseases: analysis of 1222 consecutive patients from a prospective database. Ann Surg 2004; 240: 698-708.

4. Akashi K, Mizuno S, Isaji S. Comparative study of perioperative management of hepatic resection. Dig Dis Sci 2000; 45: 1988-95.

5. Dimick JB, Wainess RM, Cowan JA, Upchurch GR, Jr., Knol JA, Colletti LM. National trends in the use and outcomes of hepatic resection. J Am Coll Surg 2004; 199: 31-8.

6. Maggard MA, O'Connell JB, Liu JH, Etzioni DA, Ko CY. Sample size calculations in surgery: are they done correctly? Surgery 2003; 134: 275-9.

7. Balasubramanian SP, Wiener M, Alshameeri Z, Tiruvoipati R, Elbourne D, Reed MW. Standards of reporting of randomized controlled trials in general surgery: can we do better? Ann Surg 2006; 244: 663-7.

8. Choti MA, Bowman HM, Pitt HA, Sosa JA, Sitzmann JV, Cameron JL, et al. Should hepatic resections be performed at high-volume referral centers? J Gastrointest Surg 1998; 2: 11-20.

9. Martin RC, 2nd, Brennan MF, Jaques DP. Quality of complication reporting in the surgical literature. Ann Surg 2002; 235: 803-13.

10. Moher D, Schulz KF, Altman DG. The CONSORT statement: revised recommendations for improving the quality of reports of parallel-group randomised trials. Lancet 2001; 357: 1191-4.

11. Terminology Committee of the IHPBA. Terminology of liver anatomy and resections. HPB Surg 2000; 2: 333-9.

12. Dindo D, Demartines N, Clavien PA. Classification of surgical complications: a new proposal with evaluation in a cohort of 6336 patients and results of a survey. Ann Surg 2004; 240: 205-13.

13. Kirkwood BR. Essentials of medical statistics. Oxford: Blackwell Scientific Publications; 1988.

14. Schafer JL, Graham JW. Missing data: our view of the state of the art. Psychol Methods 2002; 7: 147-77.

15. Senn S. Some controversies in planning and analysing multi-centre trials. Stat Med 1998; 17: 175365.

16. van Breukelen GJ, Candel MJ, Berger MP. Relative efficiency of unequal versus equal cluster sizes in cluster randomized and multicentre trials. Stat Med 2007; 26: 2589-603.

17. Rasbash J, Brown W, Goldstein H, Yang M, Plewis I, Healy M, et al. A user's guide to MLwiN. London: Multilevel Models Project, Institute of Education, University of London; 2000.

18. Hutchins R, Bertucci M. Experience with TissueLink--radiofrequency-assisted parenchymal division. Dig Surg 2007; 24: 318-21.

19. Ayav A, Jiao LR, Habib NA. Bloodless liver resection using radiofrequency energy. Dig Surg 2007; 24: 314-7.

20. Milicevic M, Bulajic P, Zuvela M, Dervenis C, Basaric D, Galun D. A radiofrequency-assisted minimal blood loss liver parenchyma dissection technique. Dig Surg 2007; 24: 306-13.

21. Watanabe I, Mayumi T, Arishima T, Takahashi H, Shikano T, Nakao A, et al. Hyperlactemia can predict the prognosis of liver resection. Shock 2007; 28: 35-8. 
22. Figueras J, Llado L, Miro M, Ramos E, Torras J, Fabregat J, et al. Application of fibrin glue sealant after hepatectomy does not seem justified: results of a randomized study in 300 patients. Ann Surg 2007; 245: 536-42.

23. Arru M, Pulitano C, Aldrighetti L, Catena M, Finazzi R, Ferla G. A prospective evaluation of ultrasonic dissector plus harmonic scalpel in liver resection. Am Surg 2007; 73: 256-60.

24. Pessaux P, Regimbeau JM, Dondero F, Plasse M, Mantz J, Belghiti J. Randomized clinical trial evaluating the need for routine nasogastric decompression after elective hepatic resection. $\mathrm{Br} J$ Surg 2007; 94: 297-303.

25. Herman P, Machado MA, Machado MC. Silkclasy: a simple way for liver transection during anatomic hepatectomies. J Surg Oncol 2007; 95: 86-9.

26. Evrard S, Becouarn Y, Brunet R, Fonck M, Larrue C, Mathoulin-Pelissier S. Could bipolar vessel sealers prevent bile leaks after hepatectomy? Langenbecks Arch Surg 2007; 392: 41-4.

27. Benzoni E, Cojutti A, Lorenzin D, Adani GL, Baccarani U, Favero A, et al. Liver resective surgery: a multivariate analysis of postoperative outcome and complication. Langenbecks Arch Surg 2007; 392: 45-54.

28. Kwon AH, Matsui Y, Kaibori M, Ha-Kawa SK. Preoperative regional maximal removal rate of technetium-99m-galactosyl human serum albumin (GSA-Rmax) is useful for judging the safety of hepatic resection. Surgery 2006; 140: 379-86.

29. Capussotti L, Ferrero A, Vigano L, Sgotto E, Muratore A, Polastri R. Bile leakage and liver resection: Where is the risk? Arch Surg 2006; 141:690-4.

30. Saiura A, Yamamoto J, Koga R, Sakamoto Y, Kokudo N, Seki M, et al. Usefulness of LigaSure for liver resection: analysis by randomized clinical trial. Am J Surg 2006; 192: 41-5.

31. Sawada T, Kita J, Rokkaku K, Kato M, Shimoda M, Kubota K. Hepatectomy in patients with nonuremic minimal renal failure. J Gastrointest Surg 2006; 10: 740-5.

32. Sun HC, Qin LX, Lu L, Wang L, Ye QH, Ren N, et al. Randomized clinical trial of the effects of abdominal drainage after elective hepatectomy using the crushing clamp method. Br J Surg 2006; 93: 422-6.

33. Lu L, Sun HC, Qin LX, Wang L, Ye QH, Ren N, et al. Abdominal drainage was unnecessary after hepatectomy using the conventional clamp crushing technique. J Gastrointest Surg 2006; 10: 302-8.

34. Wu CC, Ho WM, Cheng SB, Yeh DC, Wen MC, Liu TJ, et al. Perioperative parenteral tranexamic acid in liver tumor resection: a prospective randomized trial toward a "blood transfusion"-free hepatectomy. Ann Surg 2006; 243: 173-80.

35. Kim Yl, Chung HJ, Song KE, Hwang YJ, Lee JW, Lee YJ, et al. Evaluation of a protease inhibitor in the prevention of ischemia and reperfusion injury in hepatectomy under intermittent Pringle maneuver. Am J Surg 2006; 191: 72-6.

36. Torzilli G, Olivari N, Del Fabbro D, Gambetti A, Leoni P, Gendarini A, et al. Bilirubin level fluctuation in drain discharge after hepatectomies justifies long-term drain maintenance. Hepatogastroenterology 2005; 52: 1206-10.

37. Aldameh A, McCall JL, Koea JB. Is routine placement of surgical drains necessary after elective hepatectomy? Results from a single institution. J Gastrointest Surg 2005; 9: 667-71.

38. SchindI MJ, Redhead DN, Fearon KC, Garden OJ, Wigmore SJ. The value of residual liver volume as a predictor of hepatic dysfunction and infection after major liver resection. Gut 2005; 54: 289-96.

39. Gruttadauria S, Doria C, Vitale CH, Cintorino D, Foglieni CS, Fung JJ, et al. Preliminary report on surgical technique in hepatic parenchymal transection for liver tumors in the elderly: a lesson learned from living-related liver transplantation. J Surg Oncol 2004; 88: 229-33. 
40. Imamura H, Seyama Y, Kokudo N, Maema A, Sugawara Y, Sano K, et al. One thousand fifty-six hepatectomies without mortality in 8 years. Arch Surg 2003; 138: 1198-206.

41. Aldrighetti L, Arru M, Caterini R, Finazzi R, Comotti L, Torri G, et al. Impact of advanced age on the outcome of liver resection. World J Surg 2003; 27: 1149-54.

42. Yanaga K, Wakasugi K, Matsusaka T, Kume H. Hepatic resection without mortality at a community hospital. Int Surg 2003; 88: 87-91.

43. Bober J, Samek P. Surgery of the tumours of the liver. Bratis/ Lek Listy 2002; 103: 403-7.

44. Muratore A, Ribero D, Ferrero A, Bergero R, Capussotti L. Prospective randomized study of steroids in the prevention of ischaemic injury during hepatic resection with pedicle clamping. Br J Surg 2003; 90: 17-22.

45. Jarnagin WR, Gonen M, Fong Y, DeMatteo RP, Ben-Porat L, Little S, et al. Improvement in perioperative outcome after hepatic resection: analysis of 1,803 consecutive cases over the past decade. Ann Surg 2002; 236: 397-406.

46. Kimura F, Shimizu H, Yoshidome H, Ohtsuka M, Kato A, Yoshitomi H, et al. Circulating cytokines, chemokines, and stress hormones are increased in patients with organ dysfunction following liver resection. J Surg Res 2006; 133: 102-12.

47. Arita J, Hasegawa K, Kokudo N, Sano K, Sugawara Y, Makuuchi M. Randomized clinical trial of the effect of a saline-linked radiofrequency coagulator on blood loss during hepatic resection. $\mathrm{Br} J$ Surg 2005; 92: 954-9.

48. Kim Yl, Hwang YJ, Song KE, Yun YK, Lee JW, Chun BY. Hepatocyte protection by a protease inhibitor against ischemia/reperfusion injury of human liver. J Am Coll Surg 2002; 195: 41-50.

49. Petrowsky H, McCormack L, Trujillo M, Selzner M, Jochum W, Clavien PA. A prospective, randomized, controlled trial comparing intermittent portal triad clamping versus ischemic preconditioning with continuous clamping for major liver resection. Ann Surg 2006; 244: 921-8.

50. Kane RL, Wang J, Garrard J. Reporting in randomized clinical trials improved after adoption of the CONSORT statement. J Clin Epidemiol 2007; 60: 241-9.

51. Guller U. Surgical outcomes research based on administrative data: inferior or complementary to prospective randomized clinical trials? World J Surg 2006; 30: 255-66.

52. Ferreira-Gonzalez I, Busse JW, Heels-Ansdell D, Montori VM, Akl EA, Bryant DM, et al. Problems with use of composite end points in cardiovascular trials: systematic review of randomised controlled trials. Bmj 2007; 334: 786-92.

53. Balzan S, Belghiti J, Farges O, Ogata S, Sauvanet A, Delefosse D, et al. The "50-50 criteria" on postoperative day 5: an accurate predictor of liver failure and death after hepatectomy. Ann Surg 2005; 242: 824-8.

54. Strasberg SM, Ludbrook PA. Who oversees innovative practice? Is there a structure that meets the monitoring needs of new techniques? J Am Coll Surg 2003; 196: 938-48. 



\section{Chapter 3}

\section{Proposal for uniform definitions of clinical}

outcomes of liver surgery: results of a web-based survey

Adapted from:

van den Broek MA, van Dam RM, van Breukelen GJ, Bemelmans MH, Oussoultzoglou

E, Pessaux P, Dejong CH, Freemantle N, Olde Damink SW. Development of a composite endpoint for randomized controlled trials in liver surgery. Br J Surg 2011; 


\section{ABSTRACT}

\section{Background}

In liver surgery, high level of evidence research, such as randomized controlled trials (RCTs) using clinical outcomes as primary endpoint, is desirable but difficult to perform. Complication rates are so low that evaluation of clinical outcomes results in large sample sizes. The use of composite endpoints and conduct of meta-analyses may enable the performance of high level of evidence research in liver surgery. Their implementation is hampered by the lack of uniform definitions of complications of liver surgery. The present study aimed to develop standard definitions of liver surgery-related clinical outcomes.

\section{Methods}

A systematic literature search was performed to identify clinical endpoints of contemporary RCTs in liver surgery. A web-based survey was composed and sent to 54 international experts in HPB surgery in order to reach consensus on definitions of the most frequently used clinical outcomes. During 2 consensus meetings, the proposed definitions were adapted by the study group according to suggestions of the respondents.

\section{Results}

The literature search yielded 47 RCTs that used clinical outcomes as primary or secondary endpoint. Clear definitions of these clinical outcomes were provided in less than one-third of trial reports and varied widely. The 12 most commonly used clinical endpoints included (in descending order): operative mortality, bile leakage, blood transfusion, intra-abdominal abscess, wound infection, intra-abdominal hemorrhage, post-resectional liver failure, pneumonia, ascites, pleural effusion, sepsis, and acute renal failure. These endpoints formed the basis of the web-based survey. Thirty-one responses were collected (response rate of 57 per cent). Based on the comments of the respondents, uniform definitions of aforementioned clinical outcomes were proposed.

\section{Conclusion}

Consensus-based, standard definitions of frequently used, clinical outcomes of liver surgery are proposed. These definitions can be used to uniformly describe clinical endpoints of RCTs in liver surgery, which will facilitate the conduct of meta-analyses in the future. Furthermore, they can be used to define components of a liver surgery specific composite endpoint. 


\section{INTRODUCTION}

The decision to adopt new surgical techniques is ideally based on results of adequately powered, randomized controlled trials (RCTs) using meaningful endpoints. These endpoints may refer to clinical, efficacy or patient-reported outcomes. This evidence-based approach of surgical innovation calls for proper trial design ${ }^{1,2}$.

A critical step in the design of a trial is the selection of an appropriate primary endpoint. The optimal primary endpoint allows for unambiguous, efficient and objective evaluation of the study intervention ${ }^{3}$. In liver surgery, single component, clinical outcomes such as surgery-related mortality or specific complications are relevant primary endpoints. However, they are of limited use as their event rate is so low that evaluation results in unfeasibly large sample sizes and long inclusion periods ${ }^{4,5}$. In response, trialists can choose for the performance of meta-analyses or use of composite endpoints (CEPs) as primary endpoints.

The success of performing meta-analyses and implementing CEPs in the field of HPB surgery is, among other factors, dependent on the presence of pre-specified definitions of clinical outcomes of liver surgery ${ }^{6-8}$. Up until now, uniform definitions of many complications of liver surgery are lacking. As an example, definitions of the liver surgery specific complications bile leakage and post-resectional liver failure varied widely, which resulted in highly variable reported incidence rates ${ }^{5,8,9}$. In other medical disciplines, joint efforts already led to consensus-based proposals for standard definitions (for example ${ }^{10,11}$ ). The use of standard definitions reduces inconsistencies in trial reporting, allows for unequivocal interpretation of trial data and facilitates comparison of trial results ${ }^{8,10,12}$.

The present study was designed to develop uniform definitions of clinical outcomes of liver surgery.

\section{METHODS}

A multi-step approach was applied to obtain consensus on definitions of clinical outcomes of liver surgery. A structured literature search was conducted to identify clinical endpoints used in contemporary RCTs in liver surgery. Definitions of these clinical endpoints were extracted from the trial reports. Based on these results, a web-based survey was designed, which consisted of two parts. The first part included proposals for definitions of clinical outcomes of liver surgery. The second part focussed on the selection of components of a liver surgery specific CEP and is described in chapter 4 of the present thesis. 


\section{A. Systematic literature search to identify clinical endpoints of RCTs in liver surgery}

\section{Search strategy and study eligibility}

An electronic search was performed to identify all single component, clinical outcomes used as primary or secondary endpoints of RCTs in liver surgery. PubMed, the Cochrane Library and Embase were searched using the terms "liver resection OR hepatic resection OR hepatectomy" combined with "randomized controlled trial". Related terms were also included. The search was limited to articles that included patients older than 19 years of age and were published in English between January 2004 and December 2008. Reference lists were cross-checked for additionally eligible papers.

Studies were eligible if they (1) were RCTs, (2) reported on outcomes of liver surgery, (3) reported on human subjects, and (4) used dichotomous, clinical outcomes as either primary or secondary endpoints. Studies were excluded if they (1) reported on non liver surgery-related outcomes (were not relevant), (2) used surrogate outcomes only as primary and secondary endpoints, (3) described a study protocol of an RCT, (4) were published in abstract form only or (5) could not be retrieved.

\section{Selection and data extraction}

Abstracts of RCTs were screened by one author. Full-texts of potentially eligible RCTs were retrieved via the internet or from the corresponding author. A standard checklist was used for full-text examination, performed by two authors who decided on inclusion for final analysis. Disagreements were resolved by discussion. Data on study origin, design, sample size, study interval, type of intervention, primary and secondary endpoints, and study outcomes as well as definitions of clinical endpoints were recorded systematically. If the primary endpoint was not mentioned explicitly, the endpoint used for sample size calculations was considered the primary endpoint.

\section{B. Web-based survey on definitions of complications of liver surgery}

The clinical endpoints most frequently used in the selected RCTs formed the basis of a web-based survey. The survey was designed using commercially available survey software (www.surveymonkey.com) and consisted of two parts. The intention of this survey was (1) to reach consensus on definitions of complications of liver surgery (part I) and (2) to select components of a future liver surgery specific CEP (part II, described in chapter 4 of the present thesis). Directors of HPB units worldwide were invited to participate in the survey. The survey opened in February 2009 and closed in July 2009. Reminder e-mails were sent after 1 and 3 months and, if necessary, directors were personally contacted.

This paper deals with the results of part I of the survey. Part I consisted of questions on definitions of complications of liver surgery and their severity grade. The presented definitions were derived from the selected RCTs and other key papers in the field. Attributes 
of the proposed definitions were that they were (1) consistent, (2) self-explanatory, (3) linguistically correct, (4) precise, (5) concise, (6) time-dependent, and (7) objective (adapted from ${ }^{13}$ ). The cutoff for approval of the definition was set at more than twothirds of respondents indicating that they agreed with the proposed definition. Each proposed definition was supplemented with additional questions on complicationrelated topics and a comment box was available for replies.

Initially, severity grading was performed using a grading system designed by Ferreiro-Gonzales and colleagues ${ }^{14}$. After analysis of the first ten replies, this severity grading system was abandoned as it was considered too subjective. The more objective Clavien-Dindo severity grading system, which is based on the intervention required for a complication, was adopted thereafter ${ }^{15}$. In short, any deviation from the postoperative course without the need for pharmacological, radiological or surgical intervention was classified as Clavien-Dindo grade 1; complications requiring pharmacological treatment were graded as grade 2; complications requiring surgical or radiological intervention not under general anaesthesia were classified as grade $3 a$ and under general anaesthesia as grade $3 \mathrm{~b}$; grade 4 complications were life-threatening complications requiring intensive care because of single organ dysfunction (grade 4a) or multi-organ dysfunction (grade 4b); death was classified as grade 5 .

\section{Consensus on definitions of complications of liver surgery}

After collection of all replies, the proposed definitions were adjusted according to comments of the respondents during two meetings of the project group. The project group consisted of the authors of the present paper.

\section{RESULTS}

\section{A. Systematic literature search}

The electronic search identified 326 abstracts that fulfilled the search terms. Main reasons for exclusion are shown in Figure 3.1. No additional RCTs were identified by hand searching the reference lists. Finally, 47 RCTs were included in the analysis ${ }^{16-62}$. Their summarized characteristics are depicted in Table 3.1 and Appendix 5. Most trials were published by European $(n=26)$ or Asian $(n=20)$ centres and focussed on interventions with medication ( $n=17$ ) or new surgical techniques ( $n=15)$. Eighteen RCTs had a single component, dichotomous primary endpoint and twenty-nine a surrogate, continuous primary endpoint (Appendix 5). Mean sample size of the included RCTs was 100 patients (range 20 - 364 patients).

Table 3.2 depicts the single component, clinical endpoints that were used as either primary or secondary endpoints and the number of times they were used. A minority of 
Potentially relevant RCTs identified and screened for retrieval, $n=326$

- PubMed, $n=113$

- Cochrane, $n=190$

- Embase, $n=23$

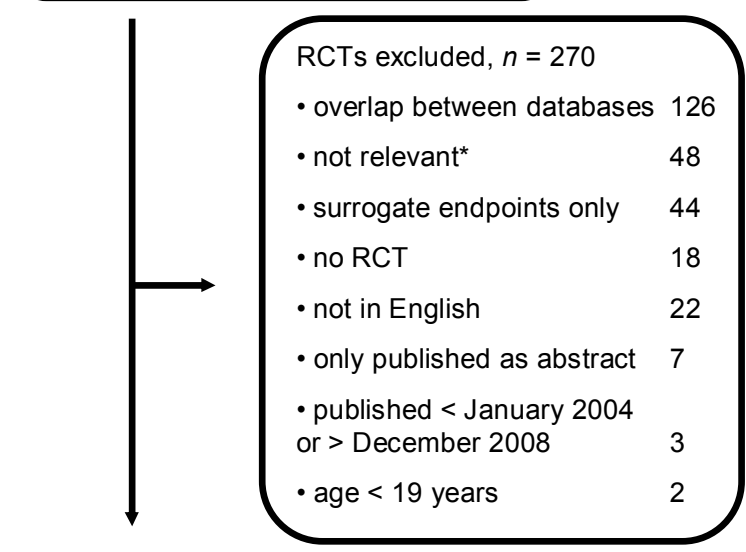

RCTs retrieved for more detailed evaluation, $n=56$

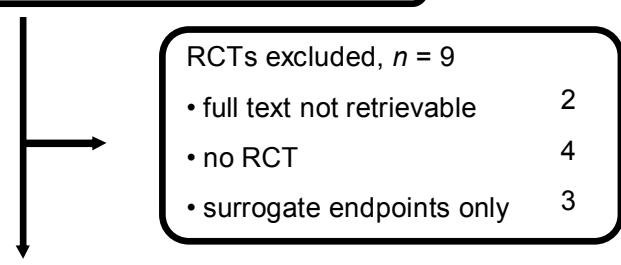

Potentially appropriate RCTs to be included in the analysis, $n=47$

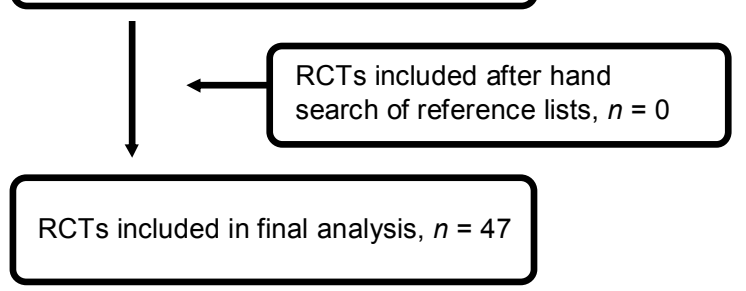

Figure 3.1 Flowchart of study selection.

Legend: RCT, randomized controlled trial; $N$, number; ${ }^{*}$ e.g. RCTs on liver transplantation or pancreatic surgery.

trial reports explicitly stated definitions of these clinical endpoints (Table 3.2), except for the endpoint mortality. A detailed overview of the definitions provided by the selected RCTs can be found in Appendix 6 . 
Table 3.1 Characteristics of the selected randomized controlled trials.

\begin{tabular}{lc}
\hline Characteristics & $\boldsymbol{N}=\mathbf{4 7}$ \\
\hline Continent of origin & $20(43)$ \\
Asia & $26(55)$ \\
Europe & $1(2)$ \\
$\quad$ North America & \\
Type of intervention & $17(36)$ \\
$\quad$ Medication & $15(32)$ \\
$\quad$ New surgical technique & $7(15)$ \\
$\quad$ Technical device & $8(17)$ \\
Other* & $5(11)$ \\
Multicentre trial & $18(38)$ \\
Clinical, dichotomous endpoint as primary endpoint & 10 \\
$\quad$ Single component endpoint & 8 \\
Composite endpoint & $29(62)$ \\
Surrogate, continuous endpoint as primary endpoint & $100(20-364)$ \\
Mean sample size (patients) (range) &
\end{tabular}

Numbers between parentheses are percentages unless otherwise indicated; $N$, number; ${ }^{*}$ e.g. type of blood transfusion.

Table 3.2 Single component, clinical outcomes used as primary or secondary endpoints in the selected randomized controlled trials $(n=47)$.

\begin{tabular}{lcc}
\hline Endpoint & $\begin{array}{c}\text { N times used as primary } \\
\text { or secondary endpoint } \\
\text { in the selected RCTs* }\end{array}$ & $\begin{array}{c}\text { N times explicitly } \\
\text { defined in the selected } \\
\text { RCTs** }\end{array}$ \\
\hline Operative, 30-day, in-hospital mortality & 38 & 34 \\
Biliary leakage, biliary fistula, bilioma & 35 & 10 \\
Blood transfusion & 34 & 0 \\
Intra-abdominal, perihepatic or subphrenic abscess & 28 & 6 \\
Wound infection, wound dehiscence & 27 & 4 \\
Intra-abdominal hemorrhage, bleeding & 25 & 1 \\
Post-resectional liver failure, hepatic dysfunction & 24 & 11 \\
Pneumonia & 23 & 4 \\
Ascites & 21 & 6 \\
Pleural effusion & 20 & 0 \\
Sepsis, septicemia & 9 & 1 \\
Acute renal failure & 7 & 1 \\
Ileus & 6 & 0 \\
Pulmonary embolus & 6 & 0 \\
Urinary tract infection & 6 & 0 \\
Atelectasis & 5 & 1 \\
\hline
\end{tabular}


Table 3.2 Continued

\begin{tabular}{lcc}
\hline Endpoint & $\begin{array}{c}\boldsymbol{N} \text { times used as primary } \\
\text { or secon-dary endpoint } \\
\text { in the selected RCTs* }\end{array}$ & $\begin{array}{c}\boldsymbol{N} \text { times explicitly } \\
\text { defined in the selected } \\
\text { RCTs** }\end{array}$ \\
\hline Myocardial infarction & 5 & 1 \\
Portal vein thrombosis & 5 & 0 \\
Gastro-intestinal perforation & 3 & 0 \\
Gastro-intestinal bleeding & 2 & 0 \\
\hline
\end{tabular}

$N$, number; RCT, randomized controlled trial; * clinical endpoints used in studies reported by the same institute using data from overlapping time periods were taken into account once (Appendix 5); ${ }^{* *}$ a complete list of definitions is provided in Appendix 6.

\section{B. Results of the web-based survey}

The 12 single component, clinical outcomes most frequently used as primary or secondary endpoints in the selected RCTs (Table 3.2) formed the basis of the webbased survey. An example of the survey can be found in Appendix 7. Fifty-four international HPB surgeons received an invitation to participate in the survey, of whom 31 (57 per cent) completed part I of the survey. Responses came from Europe $(n=27$ respondents), Asia ( $n=2)$, and North America $(n=2)$ (a complete list of respondents is provided in Appendix 8). The number of respondents agreeing with the proposed definitions is depicted in Table 3.3. Of important notice, less than two-thirds of the respondents agreed with the proposed definitions of operative mortality and intraabdominal bleeding.

\section{Consensus-based definitions of complications of liver surgery}

After 2 consensus meetings with the project group, proposed definitions of complications of liver surgery were composed. The definitions of operative mortality and intra-abdominal bleeding were considerably changed after critical review. In line with suggestions of the respondents, intra-abdominal bleeding was divided into intraabdominal hemorrhage and intra-abdominal hematoma. The final, proposed definitions are as follows:

- Operative mortality: death of a patient during or after the initial surgical procedure that is related to the surgical procedure.

- Bile leakage: any quantity of bile leaking via the abdominal wound or drains at least $48 \mathrm{hr}$ postoperatively; intra-abdominal collection of bile at the time of radiological imaging, re-operation or percutaneous drainage; cholangiographic evidence of contrast leakage. Fluid in drain or intra-abdominal collection should have a bilirubin content at least three-times the upper normal serum level in patients with postoperatively normal serum bilirubin levels, or a 50 per cent higher bilirubin level than 
Table 3.3 Number of respondents agreeing with the proposed definition of clinical outcomes of liver surgery.

\begin{tabular}{ll}
\hline Clinical outcome & $\boldsymbol{N}=\mathbf{3 1}$ \\
\hline Operative mortality & $19(61)$ \\
Bile leakage & $22(71)$ \\
Blood transfusion & $27(87)$ \\
Intra-abdominal abscess & $26(84)$ \\
Wound infection & $28(90)$ \\
Intra-abdominal bleeding & $14(45)$ \\
Post-resectional liver failure & $21(68)$ \\
Pneumonia & $29(94)$ \\
Ascites & $23(74)$ \\
Pleural effusion & $25(81)$ \\
Sepsis & $30(97)$ \\
Acute renal failure & $28(90)$ \\
\hline
\end{tabular}

The cutoff level for approval was set at more than two-thirds of the respondents indicating to agree with the proposed definition; Numbers between parentheses are percentages; $N$, number.

the serum bilirubin level in patients with postoperatively elevated serum bilirubin levels.

$\rightarrow$ Additional information: 16 respondents (52 per cent) believed that biochemical evidence of bilirubin in the drain fluid was not a prerequisite for the diagnosis bile leakage. In accordance, 17 respondents (55 per cent) did not routinely check bilirubin levels in drain fluid.

- Blood transfusion: administration of any quantity of red blood cells during the operation or within $48 \mathrm{hr}$ after surgery.

$\rightarrow$ Additional information: 16 respondents (52 per cent) indicated that not only intraoperative blood transfusions, but also administration of red blood cells after surgery (within the first 24 to $48 \mathrm{hr}$ ) should be recorded.

- Intra-abdominal abscess: any quantity of purulent fluid leaking via the abdominal drain; walled-off collection of pus in the abdominal cavity at the time of radiological imaging, re-operation or percutaneous drainage. Fluid in drain or intra-abdominal collection should be culture-positive.

$\rightarrow$ Additional information: 74 per cent of respondents ( $n=23$ respondents) indicated that a microbiological culture of the fluid should be positive, unless antibiotics had been given empirically in advance of a microbiological culture.

- Intra-abdominal hemorrhage: any quantity of blood leaking via the abdominal drain either or not accompanied by a drop in hemoglobin level; intra-abdominal active bleeding during radiological imaging or re-operation.

- Intra-abdominal hematoma: collection of blood in the proximity of the liver that can be diagnosed either radiologically or during re-operation. 
- Post-resectional liver failure: postoperative acquired failure of one or more of the hepatic excretory, detoxifying and synthetic functions that include serum bilirubin of more than $50 \mu \mathrm{mol} / \mathrm{L}$ [ $>2.9 \mathrm{mg} / \mathrm{dL}$ ], a prothrombin index below 50 per cent [international standardized ratio > 1.7] and/or hepatic encephalopathy grade 3 or 4 from postoperative day 3 onwards. Other obvious causes for the observed deterioration should be excluded (e.g. biliary obstruction).

$\rightarrow$ Additional information: according to the respondents, additional parameters suggestive of hepatic failure were changes in serum ammonia, lactate or albumin levels.

- Ascites: pathological accumulation of clear fluid in the abdominal cavity evidenced by leakage of fluid in an amount of more than $500 \mathrm{~mL} /$ day via the abdominal wound or, if present, the abdominal drain from postoperative day 3 onwards.

$\rightarrow$ Additional information: the majority of respondents (74 per cent) indicated not to check the nature of the fluid in order to differentiate between transudate and exudate.

- Pleural effusion: any fluid in the pleural cavity proven by radiological imaging

$\rightarrow$ Additional information: 25 out of 31 (82 per cent) respondents considered asymptomatic pleural effusion a normal response to liver surgery.

The majority of respondents agreed with the definitions of wound infection, pneumonia and sepsis, described by the Centres for Diseases Control and Prevention ${ }^{63}$, and acute renal failure, described by Singri and colleagues ${ }^{64}$, which are as follows:

- Wound infection ${ }^{63}$ : divided into superficial and deep incisional surgical site infection (SSI):

- Superficial incisional SSI

Infection occurs within 30 days after the operation and infection involves only skin or subcutaneous tissue of the incision and at least one of the following:

1. Purulent drainage, with or without laboratory confirmation, from the superficial incision.

2. Organisms isolated from an aseptically obtained culture of fluid or tissue from the superficial incision.

3. At least one of the following signs or symptoms of infection: pain or tenderness, localized swelling, redness, or heat, and superficial incision is deliberately opened by surgeon, unless incision is culture-negative.

4. Diagnosis of superficial incisional SSI by the surgeon or attending physician.

- Deep incisional SSI

Infection occurs within 30 days after the operation and the infection appears to be related to the operation and infection involves deep soft tissues of the incision and at least one of the following: 
1. Purulent drainage from the deep incision but not from the organ / space component of the surgical site.

2. A deep incision spontaneously dehisces or is deliberately opened by a surgeon when the patient has at least one of the following signs or symptoms: fever (temperature of more than $38^{\circ} \mathrm{C}$ ), localized pain, or tenderness, unless site is culture-negative.

3. An abscess or other evidence of infection involving the deep incision is found on direct examination, during re-operation, or by histopathological or radiologic examination.

4. Diagnosis of a deep incisional SSI by a surgeon or attending physician.

- Pneumonia63: clinical entity meeting one of the following criteria:

1. Rales or dullness to percussion on physical examination of the chest and any of the following:

a. New onset of purulent sputum or change in character of sputum.

b. Organisms isolated from blood culture.

c. Isolation of pathogens from specimen obtained by transtracheal aspirate, broncheal brushing, or biopsy.

2. Chest radiographic examination shows new or progressive infiltrate, consolidation, cavitation, or pleural effusion and any of the following:

a. New onset of purulent sputum or change in character of sputum.

b. Organisms isolated from blood culture.

c. Isolation of pathogens from specimen obtained by transtracheal aspirate, broncheal brushing, or biopsy.

d. Isolation of virus or detection of viral antigen in respiratory secretions.

e. Diagnostic single antibody titer (IgM) or fourfold increase in paired serum samples (lgG) for pathogen.

f. Histopathological evidence of pneumonia.

- Sepsis ${ }^{63}$ : the clinical syndrome defined by the presence of both infection and a systemic inflammatory response.

- Infection: pathological process caused by invasion of normally sterile tissue or fluid or body cavity by pathogenic or potentially pathogenic micro-organisms.

- Systemic inflammatory response syndrome (at least 2 criteria must be met):

1. Body temperature higher than $38^{\circ} \mathrm{C}$ or lower than $36^{\circ} \mathrm{C}$

2. Heart rate higher than $90 / \mathrm{min}$

3. Hyperventilation evidenced by respiratory rate higher than $20 /$ min or arterial carbon dioxide partial pressure lower than $32 \mathrm{mmHg}$

4. White blood cell count higher than 12,000 cells/ $\mu \mathrm{L}$ or lower than 4,000 cells $/ \mu \mathrm{L}$

- Acute renal failure ${ }^{64}$ : increase in serum creatinine of $0.5 \mathrm{mg} / \mathrm{dL}[44.2 \mu \mathrm{mol} / \mathrm{L}]$ if baseline is below $2.5 \mathrm{mg} / \mathrm{dL}$ [ $221 \mu \mathrm{mol} / \mathrm{L}]$ or an increase in serum creatinine by more than 20 per cent if baseline level is above $2.5 \mathrm{mg} / \mathrm{dL}$ [221 $\mu \mathrm{mol} / \mathrm{L}]$. 


\section{DISCUSSION}

Clear definitions of clinical outcomes, used as primary or secondary endpoints of RCTs in liver surgery, are not commonly provided. If present, definitions differ substantially among trial reports, which hampers the interpretation of trial results and compromises the validity of systematic reviews and meta-analyses. The present study was designed to develop standard definitions of clinical outcomes of liver surgery. Based on a systematic literature search and consensus among international experts in HPB surgery, definitions of clinical outcomes of liver surgery are proposed.

Consistency in study design and adequacy in study reporting are essential to allow accurate interpretation of study data and comparison of study results. Examples of initiatives leading to more uniform reporting in HPB surgery are, for example, the Brisbane 2000 system of the nomenclature of hepatic anatomy and resection ${ }^{13}$, the recommendation of endpoints for clinical trials in hepatocellular carcinoma ${ }^{12}$ and the Clavien-Dindo severity grading system ${ }^{65}$. The present systematic literature search demonstrated that consistency was lacking with regard to definitions of clinical endpoints of RCTs in liver surgery. Inconsistent definitions are undesirable as they are not reproducible. The conduct of systematic reviews and meta-analyses is hindered by the lack of consistency, while combining results from different RCTs is becoming increasingly important in the field of HPB surgery. This may be due to the low feasibility of conducting adequately powered RCTs using single component, clinical endpoints as primary endpoints as a consequence of declining event rates after liver surgery ${ }^{5,66}$.

The present comprehensive literature search showed that the mean sample size of RCTs in liver surgery was 100 patients. A previous study demonstrated that, due to the low rates of complications of liver surgery, sample sizes ranging between 826 and 6250 patients per study arm would be needed for an adequately powered RCT aiming to show a 50 per cent relative reduction of a single component, primary endpoint ${ }^{5}$. As a consequence, many of the RCTs included in the present systematic review are underpowered with respect to single component, clinical outcomes and their conclusions should be interpreted with caution. Problems with the quality and sample size of RCTs in liver surgery have been reported before ${ }^{8,67,68}$. Larger sample sizes can be obtained by the conduct of multicentre trials, institution of national databases and performance of meta-analyses ${ }^{1,69}$.

The implementation of a liver surgery specific CEP might enhance the feasibility of liver surgery-related RCTs using clinical outcomes as primary endpoint. A CEP increases the statistical power of an RCT by capturing the number of patients experiencing any one of several clinical outcomes within a specific timeframe ${ }^{70,71}$. To the best of our knowledge, a well-defined CEP to be used as primary endpoint of RCTs in liver surgery is lacking. A first step in the design of a CEP is achieving consensus on definitions of its 
components. The definitions proposed in the present paper can be used to accurately describe the components of a future liver surgery specific CEP.

Simultaneously to the efforts of our group, the International Study Group of Liver Surgery (ISGLS) developed consensus definitions of the complications post-hepatectomy liver failure, bile leakage and post-hepatectomy hemorrhage $\mathrm{e}^{72-74}$. The definitions proposed by the ISGLS resemble those presented in the present paper. An advantage of the ISGLS definitions is their retrospective validation in large patient cohorts. On the other hand, a remarkable difference exists between their work and the present study concerning the aspect of severity grading. Stratification of the severity of complications is important as it allows for judicious interpretation of data. Based on the results of our survey and preference of the respondents, the objective Clavien-Dindo classification for severity grading of surgical complications was selected ${ }^{15,75,76}$, whereas the ISGLS proposes to use a newly designed classification system. The Clavien-Dindo severity grading system is widely used in surgical literature, allows for objective grading of complications, and reflects the severity of a complication from the doctors' and patients' perspective ${ }^{65}$. The grading system proposed by the ISGLS may introduce confusion as it does not adhere to the Clavien-Dindo grades, which are familiar to most HPB surgeons. Moreover, it introduces subjectivity by, for example, stating that Grade A bile leakage implies "no or little change in patients' clinical management"72. In order to use and interpret CEPs accurately, objective severity grading is important as it has been proven that CEPs can be misleading if components vary widely in clinical relevance ${ }^{7,14,77}$. In fact, components with low clinical relevance tend to have a higher event rate and treatment effect ${ }^{14}$.

To conclude, the proposed definitions of clinical outcomes of liver surgery can be used to uniformly describe clinical endpoints of RCTs. The presence of standard definitions of complications of liver surgery enables the conduct of systematic reviews and meta-analyses in HPB surgery and forms the first step in the process of developing a well-defined liver surgery specific CEP. Selection of components of a future liver surgery specific CEP is the next challenge to meet. 


\section{REFERENCES}

1. McCulloch P, Altman DG, Campbell WB, Flum DR, Glasziou P, Marshall JC, et al. No surgical innovation without evaluation: the IDEAL recommendations. Lancet 2009; 374: 1105-12.

2. Schulz KF, Altman DG, Moher D. CONSORT 2010 statement: updated guidelines for reporting parallel group randomised trials. Bmj 2010; 340: 698-702.

3. Buzney EA, Kimball AB. A critical assessment of composite and coprimary endpoints: a complex problem. J Am Acad Dermatol 2008; 59: 890-6.

4. Washburn K. Endpoints in trials for clinical liver transplantation. Curr Opin Organ Transplant 2008; 13: 252-6.

5. van den Broek MA, van Dam RM, Malago M, Dejong CH, van Breukelen GJ, Olde Damink SW. Feasibility of randomized controlled trials in liver surgery using surgery-related mortality or morbidity as endpoint. Br J Surg 2009; 96: 1005-1014.

6. Ferreira-Gonzalez I, Permanyer-Miralda G, Busse JW, Bryant DM, Montori VM, Alonso-Coello P, et al. Methodologic discussions for using and interpreting composite endpoints are limited, but still identify major concerns. J Clin Epidemiol 2007; 60: 651-7.

7. Neaton JD, Gray G, Zuckerman BD, Konstam MA. Key issues in end point selection for heart failure trials: composite end points. J Card Fail 2005; 11: 567-75.

8. Martin RC, 2nd, Brennan MF, Jaques DP. Quality of complication reporting in the surgical literature. Ann Surg 2002; 235: 803-13.

9. van den Broek MA, Olde Damink SW, Dejong $\mathrm{CH}$, Lang $\mathrm{H}$, Malago M, Jalan R, et al. Liver failure after partial hepatic resection: definition, pathophysiology, risk factors and treatment. Liver Int 2008; 28: 767-80.

10. Hudis CA, Barlow WE, Costantino JP, Gray RJ, Pritchard KI, Chapman JA, et al. Proposal for standardized definitions for efficacy end points in adjuvant breast cancer trials: the STEEP system. $J$ Clin Oncol 2007; 25: 2127-32.

11. Bassi C, Dervenis C, Butturini G, Fingerhut A, Yeo C, Izbicki J, et al. Postoperative pancreatic fistula: an international study group (ISGPF) definition. Surgery 2005; 138: 8-13.

12. Llovet JM, Di Bisceglie AM, Bruix J, Kramer BS, Lencioni R, Zhu AX, et al. Design and endpoints of clinical trials in hepatocellular carcinoma. J Natl Cancer Inst 2008; 100: 698-711.

13. Strasberg SM. Nomenclature of hepatic anatomy and resections: a review of the Brisbane 2000 system. J Hepatobiliary Pancreat Surg 2005; 12: 351-5.

14. Ferreira-Gonzalez I, Busse JW, Heels-Ansdell D, Montori VM, AkI EA, Bryant DM, et al. Problems with use of composite end points in cardiovascular trials: systematic review of randomised controlled trials. Bmj 2007; 334: 786-92.

15. Dindo D, Demartines N, Clavien PA. Classification of surgical complications: a new proposal with evaluation in a cohort of 6336 patients and results of a survey. Ann Surg 2004; 240: 205-13.

16. Beck-Schimmer B, Breitenstein S, Urech S, De Conno E, Wittlinger M, Puhan M, et al. A randomized controlled trial on pharmacological preconditioning in liver surgery using a volatile anesthetic. Ann Surg 2008; 248: 909-18.

17. Jarnagin WR, Gonen M, Maithel SK, Fong Y, D'Angelica MI, Dematteo RP, et al. A prospective randomized trial of acute normovolemic hemodilution compared to standard intraoperative management in patients undergoing major hepatic resection. Ann Surg 2008; 248: 360-9.

18. Torzilli G, Donadon M, Marconi M, Procopio F, Palmisano A, Del Fabbro D, et al. Monopolar floating ball versus bipolar forceps for hepatic resection: a prospective randomized clinical trial. $J$ Gastrointest Surg 2008; 12: 1961-6. 
19. Ko JS, Gwak MS, Choi SJ, Kim GS, Kim JA, Yang M, et al. The effects of desflurane and propofolremifentanil on postoperative hepatic and renal functions after right hepatectomy in liver donors. Liver Transp/ 2008; 14: 1150-8.

20. Heizmann O, Loehe F, Volk A, Schauer RJ. Ischemic preconditioning improves postoperative outcome after liver resections: a randomized controlled study. Eur J Med Res 2008; 13: 79-86.

21. Nordlinger B, Sorbye H, Glimelius B, Poston GJ, Schlag PM, Rougier P, et al. Perioperative chemotherapy with FOLFOX4 and surgery versus surgery alone for resectable liver metastases from colorectal cancer (EORTC Intergroup trial 40983): a randomised controlled trial. Lancet 2008; 371 : 1007-16.

22. Yuksel O, Akyurek N, Sahin T, Salman B, Azili C, Bostanci H. Efficacy of radical surgery in preventing early local recurrence and cavity-related complications in hydatic liver disease. $J$ Gastrointest Surg 2008; 12: 483-9.

23. Kim YI, Fujita S, Hwang YJ, Chun JM, Song KE, Chun BY. Successful intermittent application of the Pringle maneuver for 30 minutes during human hepatectomy: a clinical randomized study with use of a protease inhibitor. Hepatogastroenterology 2007; 54: 2055-60.

24. Pulitano C, Aldrighetti L, Arru M, Finazzi R, Soldini L, Catena M, et al. Prospective randomized study of the benefits of preoperative corticosteroid administration on hepatic ischemia-reperfusion injury and cytokine response in patients undergoing hepatic resection. HPB (Oxford) 2007; 9: 183-9.

25. Schmidt SC, Hamann S, Langrehr JM, Hoflich C, Mittler J, Jacob D, et al. Preoperative high-dose steroid administration attenuates the surgical stress response following liver resection: results of a prospective randomized study. J Hepatobiliary Pancreat Surg 2007; 14: 484-92.

26. Pulitano C, Aldrighetti L, Arru M, Finazzi R, Catena M, Guzzetti E, et al. Preoperative methylprednisolone administration maintains coagulation homeostasis in patients undergoing liver resection: importance of inflammatory cytokine modulation. Shock 2007; 28: 401-5.

27. Campagnacci R, De Sanctis A, Baldarelli M, Di Emiddio M, Organetti L, Nisi M, et al. Hepatic resections by means of electrothermal bipolar vessel device (EBVS) LigaSure V: early experience. Surg Endosc 2007; 21: 2280-4.

28. Hashimoto T, Kokudo N, Orii R, Seyama Y, Sano K, Imamura H, et al. Intraoperative blood salvage during liver resection: a randomized controlled trial. Ann Surg 2007; 245: 686-91.

29. Kostopanagiotou G, Pandazi A, Matsota P, Arkadopoulos N, Dalamanga N, Politou M, et al. Effect of packed red blood cells transfusion on plasma fibronectin during liver resection. Transfus Med 2007; 17: 115-8.

30. Figueras J, Llado L, Miro M, Ramos E, Torras J, Fabregat J, et al. Application of fibrin glue sealant after hepatectomy does not seem justified: results of a randomized study in 300 patients. Ann Surg 2007; 245: 536-42.

31. Togo S, Tanaka K, Matsuo K, Nagano Y, Ueda M, Morioka D, et al. Duration of antimicrobial prophylaxis in patients undergoing hepatectomy: a prospective randomized controlled trial using flomoxef. J Antimicrob Chemother 2007; 59: 964-70.

32. Lupo L, Gallerani A, Panzera P, Tandoi F, Di Palma G, Memeo V. Randomized clinical trial of radiofrequency-assisted versus clamp-crushing liver resection. Br J Surg 2007; 94: 287-91.

33. Pessaux P, Regimbeau JM, Dondero F, Plasse M, Mantz J, Belghiti J. Randomized clinical trial evaluating the need for routine nasogastric decompression after elective hepatic resection. $\mathrm{Br} J$ Surg 2007; 94: 297-303. 
34. Shi M, Guo RP, Lin XJ, Zhang YQ, Chen MS, Zhang CQ, et al. Partial hepatectomy with wide versus narrow resection margin for solitary hepatocellular carcinoma: a prospective randomized trial. Ann Surg 2007; 245: 36-43.

35. Smyrniotis V, Theodoraki K, Arkadopoulos N, Fragulidis G, Condi-Pafiti A, Plemenou-Fragou M, et al. Ischemic preconditioning versus intermittent vascular occlusion in liver resections performed under selective vascular exclusion: a prospective randomized study. Am J Surg 2006; 192: 669-74.

36. Petrowsky H, McCormack L, Trujillo M, Selzner M, Jochum W, Clavien PA. A prospective, randomized, controlled trial comparing intermittent portal triad clamping versus ischemic preconditioning with continuous clamping for major liver resection. Ann Surg 2006; 244: 921-8.

37. Sugawara G, Nagino M, Nishio H, Ebata T, Takagi K, Asahara T, et al. Perioperative synbiotic treatment to prevent postoperative infectious complications in biliary cancer surgery: a randomized controlled trial. Ann Surg 2006; 244: 706-14.

38. Liu CL, Fan ST, Cheung ST, Lo CM, Ng IO, Wong J. Anterior approach versus conventional approach right hepatic resection for large hepatocellular carcinoma: a prospective randomized controlled study. Ann Surg 2006; 244: 194-203.

39. Esaki M, Sano T, Shimada K, Sakamoto Y, Takahashi Y, Wakai K, et al. Randomized clinical trial of hepatectomy using intermittent pedicle occlusion with ischaemic intervals of 15 versus 30 minutes. Br J Surg 2006; 93: 944-51.

40. Saiura A, Yamamoto J, Koga R, Sakamoto Y, Kokudo N, Seki M, et al. Usefulness of LigaSure for liver resection: analysis by randomized clinical trial. Am J Surg 2006; 192: 41-5.

41. Aldrighetti L, Pulitano C, Arru M, Finazzi R, Catena M, Soldini L, et al. Impact of preoperative steroids administration on ischemia-reperfusion injury and systemic responses in liver surgery: a prospective randomized study. Liver Transpl 2006; 12: 941-9.

42. Capussotti L, Muratore A, Ferrero A, Massucco P, Ribero D, Polastri R. Randomized clinical trial of liver resection with and without hepatic pedicle clamping. Br J Surg 2006; 93: 685-9.

43. Chen XP, Zhang ZW, Zhang BX, Chen YF, Huang ZY, Zhang WG, et al. Modified technique of hepatic vascular exclusion: effect on blood loss during complex mesohepatectomy in hepatocellular carcinoma patients with cirrhosis. Langenbecks Arch Surg 2006; 391: 209-15.

44. Wang WD, Liang LJ, Huang XQ, Yin XY. Low central venous pressure reduces blood loss in hepatectomy. World J Gastroenterol 2006; 12: 935-9.

45. Sun HC, Qin LX, Lu L, Wang L, Ye QH, Ren N, et al. Randomized clinical trial of the effects of abdominal drainage after elective hepatectomy using the crushing clamp method. Br J Surg 2006; 93: 422-6.

46. Shao YF, Yang JM, Chau GY, Sirivatanauksorn Y, Zhong SX, Erhardtsen E, et al. Safety and hemostatic effect of recombinant activated factor VII in cirrhotic patients undergoing partial hepatectomy: a multicenter, randomized, double-blind, placebo-controlled trial. Am J Surg 2006; 191: 245-9.

47. Wu CC, Ho WM, Cheng SB, Yeh DC, Wen MC, Liu TJ, et al. Perioperative parenteral tranexamic acid in liver tumor resection: a prospective randomized trial toward a "blood transfusion"-free hepatectomy. Ann Surg 2006; 243: 173-80.

48. Azoulay $\mathrm{D}$, Lucidi $\mathrm{V}$, Andreani $\mathrm{P}$, Maggi $\mathrm{U}$, Sebagh $\mathrm{M}$, Ichai $\mathrm{P}$, et al. Ischemic preconditioning for major liver resection under vascular exclusion of the liver preserving the caval flow: a randomized prospective study. J Am Coll Surg 2006; 202: 203-11.

49. Kim Yl, Chung HJ, Song KE, Hwang YJ, Lee JW, Lee YJ, et al. Evaluation of a protease inhibitor in the prevention of ischemia and reperfusion injury in hepatectomy under intermittent Pringle maneuver. Am J Surg 2006; 191: 72-6. 
50. Yoshida H, Mamada Y, Taniai N, Mizuguchi Y, Shimizu T, Takahashi T, et al. Fixation of the greater omentum for prevention of delayed gastric emptying after left-sided hepatectomy: a randomized controlled trial. Hepatogastroenterology 2005; 52: 1334-7.

51. Arita J, Hasegawa K, Kokudo N, Sano K, Sugawara Y, Makuuchi M. Randomized clinical trial of the effect of a saline-linked radiofrequency coagulator on blood loss during hepatic resection. $\mathrm{Br} J$ Surg 2005; 92: 954-9.

52. Figueras J, Llado L, Ruiz D, Ramos E, Busquets J, Rafecas A, et al. Complete versus selective portal triad clamping for minor liver resections: a prospective randomized trial. Ann Surg 2005; 241 : 582-90.

53. Smyrniotis V, Arkadopoulos N, Kostopanagiotou G, Farantos C, Vassiliou J, Contis J, et al. Sharp liver transection versus clamp crushing technique in liver resections: a prospective study. Surgery 2005; 137: 306-11.

54. Kanazawa H, Nagino M, Kamiya S, Komatsu S, Mayumi T, Takagi K, et al. Synbiotics reduce postoperative infectious complications: a randomized controlled trial in biliary cancer patients undergoing hepatectomy. Langenbecks Arch Surg 2005; 390: 104-13.

55. Lodge JP, Jonas S, Oussoultzoglou E, Malago M, Jayr C, Cherqui D, et al. Recombinant coagulation factor VIla in major liver resection: a randomized, placebo-controlled, double-blind clinical trial. Anesthesiology 2005; 102: 269-75.

56. Frilling A, Stavrou GA, Mischinger HJ, de Hemptinne B, Rokkjaer M, Klempnauer J, et al. Effectiveness of a new carrier-bound fibrin sealant versus argon beamer as haemostatic agent during liver resection: a randomised prospective trial. Langenbecks Arch Surg 2005; 390: 114-20.

57. Lesurtel M, Selzner M, Petrowsky H, McCormack L, Clavien PA. How should transection of the liver be performed?: a prospective randomized study in 100 consecutive patients: comparing four different transection strategies. Ann Surg 2005; 242: 814-22, discussion 822-3.

58. Schwartz M, Madariaga J, Hirose R, Shaver TR, Sher L, Chari R, et al. Comparison of a new fibrin sealant with standard topical hemostatic agents. Arch Surg 2004; 139: 1148-54.

59. Li SQ, Liang LJ, Huang JF, Li Z. Ischemic preconditioning protects liver from hepatectomy under hepatic inflow occlusion for hepatocellular carcinoma patients with cirrhosis. World J Gastroenterol 2004; 10: 2580-4.

60. Fuster J, Llovet JM, Garcia-Valdecasas JC, Grande L, Fondevila C, Vilana R, et al. Abdominal drainage after liver resection for hepatocellular carcinoma in cirrhotic patients: a randomized controlled study. Hepatogastroenterology 2004; 51: 536-40.

61. Nuzzo G, Giuliante F, Vellone M, De Cosmo G, Ardito F, Murazio M, et al. Pedicle clamping with ischemic preconditioning in liver resection. Liver Transp/ 2004; 10: S53-7.

62. Liu CL, Fan ST, Lo CM, Wong Y, Ng IO, Lam CM, et al. Abdominal drainage after hepatic resection is contraindicated in patients with chronic liver diseases. Ann Surg 2004; 239: 194-201.

63. Garner JS, Jarvis WR, Emori TG, Horan TC, Hughes JM. CDC definitions for nosocomial infections, 1988. Am J Infect Control 1988; 16: 128-40.

64. Singri N, Ahya SN, Levin ML. Acute renal failure. Jama 2003; 289: 747-51.

65. Clavien PA, Barkun J, de Oliveira ML, Vauthey JN, Dindo D, Schulick RD, et al. The Clavien-Dindo classification of surgical complications: five-year experience. Ann Surg 2009; 250: 187-96.

66. Strasberg SM, Ludbrook PA. Who oversees innovative practice? Is there a structure that meets the monitoring needs of new techniques? J Am Coll Surg 2003; 196: 938-48.

67. Kaido T. Analysis of randomized controlled trials on hepatopancreatic surgery. Dig Dis Sci 2006; 51: 1761-6.

68. Gluud C. The culture of designing hepato-biliary randomised trials. J Hepatol 2006; 44: 607-15. 
69. Pitt HA, Kilbane M, Strasberg SM, PawlikTM, Dixon E, Zyromski NJ, et al. ACS-NSQIP has the potential to create an HPB-NSQIP option. HPB (Oxford) 2009; 11: 405-13.

70. Ross S. Composite outcomes in randomized clinical trials: arguments for and against. Am J Obstet Gynecol 2007; 196: 119 e1-6.

71. Freemantle N, Calvert M, Wood J, Eastaugh J, Griffin C. Composite outcomes in randomized trials: greater precision but with greater uncertainty? Jama 2003; 289: 2554-9.

72. Koch M, Garden OJ, Padbury R, Rahbari NN, Adam R, Capussotti L, et al. Bile leakage after hepatobiliary and pancreatic surgery: a definition and grading of severity by the International Study Group of Liver Surgery. Surgery 2011; 149: 680-8.

73. Rahbari NN, Garden OJ, Padbury R, Brooke-Smith M, Crawford M, Adam R, et al. Posthepatectomy liver failure: a definition and grading by the International Study Group of Liver Surgery (ISGLS). Surgery $2011 ; 149:$ 713-24.

74. Rahbari NN, Garden OJ, Padbury R, Maddern G, Koch M, Hugh TJ, et al. Post-hepatectomy haemorrhage: a definition and grading by the International Study Group of Liver Surgery (ISGLS). HPB (Oxford) 2011; 13: 528-35.

75. Clavien PA, Camargo CA, Jr., Croxford R, Langer B, Levy GA, Greig PD. Definition and classification of negative outcomes in solid organ transplantation. Application in liver transplantation. Ann Surg 1994; 220: 109-20.

76. Clavien PA, Sanabria JR, Strasberg SM. Proposed classification of complications of surgery with examples of utility in cholecystectomy. Surgery 1992; 111: 518-26.

77. Bethel MA, Holman R, Haffner SM, Califf RM, Huntsman-Labed A, Hua TA, et al. Determining the most appropriate components for a composite clinical trial outcome. Am Heart J 2008; 156: 63340. 


\section{Chapter 4}

\section{Development of a composite endpoint for randomized controlled trials in liver surgery}

Adapted from:

van den Broek MA, van Dam RM, van Breukelen GJ, Bemelmans MH, Oussoultzoglou

E, Pessaux P, Dejong CH, Freemantle N, Olde Damink SW. Development of a composite endpoint for randomized controlled trials in liver surgery. Br J Surg 2011; 


\section{ABSTRACT}

\section{Background}

The feasibility of randomized controlled trials (RCTs) in liver surgery using a single component, clinical endpoint is low as such endpoints require large sample sizes owing to their low incidence. A liver surgery specific composite endpoint (CEP) could solve this problem. The aim of this study was to develop a liver surgery specific CEP.

\section{Methods}

Components of a liver surgery specific CEP were selected based on a systematic literature search and consensus among international HPB surgeons. As an example, two prospective cohorts of patients who had undergone liver surgery in high-volume HPB centres were used to assess the event rate of the proposed liver surgery specific CEP and effect of its implementation on the theoretical sample size of a future RCT.

\section{Results}

Components selected for the liver surgery specific CEP were ascites, post-resectional liver failure, bile leakage, intra-abdominal hemorrhage, intra-abdominal abscess and operative mortality, all with a Clavien-Dindo grade of 3 or more and occurring within 90 days after initial liver surgery. The incidence of this liver surgery specific CEP was 19 per cent in one cohort and 11 per cent in the other. These rates led to an approximate twofold reduction in the sample size required for an adequately powered RCT in liver surgery using the liver surgery specific CEP instead of a single component, clinical endpoint as primary endpoint.

\section{Conclusion}

The proposed liver surgery specific CEP consists of ascites, post-resectional liver failure, bile leakage, intra-abdominal hemorrhage, intra-abdominal abscess and operative mortality. It has a considerably higher event rate than any of its individual components. Its use as a primary endpoint will increase the feasibility and comparability of RCTs in liver surgery. 


\section{INTRODUCTION}

In the current era of evidence-based practice, randomized controlled trials (RCTs) deliver the highest level of evidence. Therefore, their conclusions are frequently incorporated into treatment guidelines. Few RCTs have been performed in liver surgery'. This low number may be explained by the large sample size needed for an adequately powered trial using clinically relevant primary endpoints such as liver surgery-related mortality or specific types of morbidity ${ }^{2}$. These large sample sizes present a dilemma to clinical investigators who want to assess the efficacy of interventions in liver surgery on single component, clinical outcomes.

The primary endpoint of an RCT is dependent on its objective and must be chosen carefully a priori ${ }^{3}$. This is particularly important as it is the primary endpoint alone that should be used to judge whether a treatment has been effective in a trial ${ }^{4}$. Clinical outcomes such as surgery-related mortality or specific types of morbidity are important for the evaluation of the efficacy of surgical interventions. However, they are of limited use in future RCTs in liver surgery as their event rate is so low that large sample sizes are needed for adequately powered trials ${ }^{2,5}$. A possible solution would be the implementation of a well-defined composite endpoint (CEP) to be used as the primary endpoint of RCTs in liver surgery.

A CEP is a combination of two or more procedure specific complications that are considered as a single, dichotomous outcome ${ }^{6}$. CEPs lead to an increased statistical power and thus efficiency because their event rate is higher than that of a single component, clinical endpoint. Moreover, CEPs enhance the comparability of trial results, which facilitates meta-analyses, and take into account multiple outcomes or even multiple types of outcomes (for example patient-reported and clinical endpoints), thereby evaluating the overall benefit of a single intervention ${ }^{7}$. In order to do so, the individual components of a CEP need to be well-defined and clinically relevant outcome parameters.

Hitherto, CEPs have hardly been used as the primary endpoint of RCTs in liver surgery, and uniformity in components is lacking. The aim of the present study was to develop a standardized liver surgery specific CEP that can be used as primary endpoint of RCTs in liver surgery.

\section{METHODS}

A three-step approach was applied for the development of a liver surgery specific CEP. First, a systematic review was undertaken to identify frequently used dichotomous, clinical endpoints of RCTs in liver surgery (development step 1). These endpoints formed the basis of a web-based survey that aimed at reaching consensus on the definition of 
complications of liver surgery (development step 2) and at selecting components of the liver surgery specific CEP (development step 3). Development steps 1 and 2 have been described in chapter 3 of the present thesis.

The present paper deals with the selection of components of the liver surgery specific CEP (development step 3). As an example, the event rate of the proposed liver surgery specific CEP was assessed in two prospective cohorts of patients who had undergone liver surgery in high-volume European HPB centres, and the theoretical sample size of an RCT using the liver surgery specific CEP as the primary endpoint was calculated.

\section{Development step 1. Identification of single component, clinical endpoints of randomized controlled trials in liver surgery}

An electronic search was performed to identify all single component, clinical outcomes used as primary or secondary endpoints of RCTs in liver surgery published between 1 January 2004 and 31 December 2008. The results if this search have been presented in chapter 3 of the present thesis.

\section{Development step 2. Consensus on definitions of complications of liver surgery}

The endpoints most frequently used in the identified RCTs formed the basis of a webbased survey. An example of the survey can be found in Appendix 7. The survey consisted of two parts. Definitions of complications of liver surgery were proposed in part I of the survey (described in chapter 3 of the present thesis).

\section{Development step 3. Selection of components of a liver surgery specific CEP}

Part II of the survey consisted of questions on possible components of the liver surgery specific CEP. Complications preferred for inclusion in the liver surgery specific CEP by at least two-thirds of respondents were considered as components. For the final selection, additional important attributes of CEP components were taken into account ${ }^{7,8}$. In short, each component of the CEP should preferably (1) be easily ascertainable (i.e. presence of a standardized definition), (2) be of substantial clinical relevance (i.e. reflecting ClavienDindo severity grade 3 to $5^{9}$ ), and (3) have the same expected direction of treatment effect.

\section{Evaluation of the event rate of the liver surgery specific CEP}

The event rate of the individual components and the liver surgery specific CEP was determined using two prospective databases consisting of consecutive patients who had undergone partial liver resection between January 2000 and October 2009. These data sets were derived from the Department of Surgery of Maastricht University Medical Centre (Maastricht, the Netherlands) and the Division of HPB Surgery of Hautepierre University Hospital (Strasbourg, France), both high-volume European HPB centres. 
Patients who had concomitant gastrointestinal procedures or bilio-enteric anastomoses were excluded. The occurrence of an event within 90 days of initial liver surgery was determined and graded using the Clavien-Dindo classification ${ }^{9}$.

\section{Statistical analysis and sample size calculation}

Continuous data were expressed as mean (standard deviation) when normally distributed and median (range) when non-normally distributed. SPSS version 15.0 for Windows (SPSS Inc., Chicago, IL, USA) was used for statistical analysis.

The theoretical sample size of an adequately powered RCT was calculated as the minimum number of patients necessary in each arm of the trial, with $\alpha=0.05$ and $\beta$ $=0.20$, using two-sided testing. The formula used for sample size calculations can be found in Appendix 2. The sample size was calculated assuming a one-third, one-half or two-thirds relative reduction in the incidence of either a single component endpoint or the proposed liver surgery specific CEP.

\section{RESULTS}

\section{Development step 1. Identification of single component, clinical endpoints of RCTs in liver surgery}

The electronic search identified 326 abstracts that fulfilled the search terms. After selection, 47 RCTs were included in the final analysis (reference list and study details are provided in chapter 3 of the present thesis and Appendix 5). Eighteen RCTs (38 per cent) had a single component, dichotomous primary endpoint and twenty-nine (62 per cent) a surrogate, continuous primary endpoint. Eight RCTs (17 per cent) used a CEP as primary endpoint, which consisted of postoperative complications in 4 RCTs, and overall intraabdominal complications, ischemia-related complications, abdominal cavity-related complications and surgical site infections in 1 RCT each (Appendix 5). The twelve single component, clinical endpoints most frequently used in RCTs in liver surgery were: operative mortality $(n=38)$, bile leakage $(n=35)$, blood transfusion $(n=34)$, intra-abdominal abscess $(n=28)$, wound infection $(n=27)$, intra-abdominal hemorrhage $(n=25)$, postresectional liver failure $(n=24)$, pneumonia $(n=23)$, ascites $(n=21)$, pleural effusion $(n$ $=20)$, sepsis $(n=9)$, and acute renal failure $(n=7)$. These complications formed the basis of part I of the electronic survey.

\section{Development step 2. Consensus on definitions of complications of liver surgery}

Fifty-four HPB surgeons received an invitation to participate in the survey, of whom 31 (57 per cent) completed part I of the survey on definitions of complications of liver surgery. A complete list of respondents is provided in Appendix 8. The proposed defini- 
Table 4.1 Selection of components of the liver surgery specific composite endpoint.

\begin{tabular}{lcc}
\hline Complication & $\begin{array}{c}\text { N respondents considering } \\
\text { the complication liver surgery } \\
\text { specific }(\boldsymbol{n}=\mathbf{3 1})\end{array}$ & $\begin{array}{c}\text { N respondents indicating the } \\
\text { complication should be included } \\
\text { in the liver surgery specific CEP } \\
\text { ( } \mathbf{n}=\mathbf{2 8})\end{array}$ \\
\hline Pneumonia & $3(10)$ & $12(43)$ \\
Wound infection & $18(58)$ & $7(25)$ \\
Pleural effusion & $10(32)$ & $9(32)$ \\
Blood transfusion & $13(42)$ & $20(71)$ \\
Intra-abdominal abscess & $13(42)$ & $20(71)$ \\
Intra-abdominal hemorrhage & $31(100)$ & $23(82)$ \\
Bile leakage & $23(74)$ & $28(100)$ \\
Ascites & $3(10)$ & $25(89)$ \\
Sepsis & $29(94)$ & $17(61)$ \\
Post-resectional liver failure & $3(10)$ & $28(100)$ \\
Acute renal failure & n.a. & $14(50)$ \\
Operative mortality & $24(86)$
\end{tabular}

Numbers in parentheses are percentages; $N$, number; CEP, composite endpoint; n.a., not applicable.

tions of the most frequently used complications have been described in chapter 3 of the present thesis. Bile leakage, post-resectional liver failure and ascites were regarded as liver surgery specific complications by 31,29 and 23 respondents, respectively (Table 4.1).

\section{Development step 3. Selection of components of the liver surgery specific CEP}

Twenty-eight (52 per cent) HPB surgeons completed part II of the survey. Their preferences regarding the components of the liver surgery specific CEP are shown in Table 4.1. As a result, six complications with Clavien-Dindo grade 3 or more and preferred by at least two-thirds of the respondents were selected as components: ascites, postresectional liver failure, bile leakage, intra-abdominal abscess, intra-abdominal hemorrhage, and operative mortality. Although blood transfusion had a preference rate of 71 per cent, it was not included as it is regarded as a complication with a Clavien-Dindo grade of less than 3 . All respondents indicated that they would be interested in using a liver surgery specific CEP as primary endpoint of an RCT.

\section{Evaluation of the event rate of the liver surgery specific CEP}

Between January 2000 and October 2009, 340 and 583 adult patients underwent partial liver resection at Maastricht University Medical Centre and University Hospital of Strasbourg, respectively. Of these, 49 and 69 patients were excluded because of concomitant gastrointestinal procedures or bilio-enteric anastomoses. Consequently, 291 and 514 
Table 4.2 Patient characteristics and event rates of complications of liver surgery at Maastricht University Medical Centre and University Hospital of Strasbourg.

\begin{tabular}{|c|c|c|}
\hline & $\begin{array}{c}\text { Maastricht University Medical } \\
\text { Centre }(n=291)\end{array}$ & $\begin{array}{l}\text { University Hospital of Strasbourg } \\
\qquad(n=514)\end{array}$ \\
\hline Sex (female : male) & $133: 158$ & $229: 285$ \\
\hline Age (years)* & $60.0 \pm 12.8$ & $59.5 \pm 12.6$ \\
\hline Major hepatectomy ${ }^{\dagger}$ & $114(39.2)$ & $253(49.2)$ \\
\hline Complication grade $1-2^{\ddagger}$ & Incidence (\%) & Incidence (\%) \\
\hline Ascites & $2(0.7)$ & $22(4.3)$ \\
\hline Post-resectional liver failure & $8(2.7)$ & $11(2.1)$ \\
\hline Bile leakage & $1(0.3)$ & $7(1.4)$ \\
\hline Intra-abdominal abscess & $2(0.7)$ & $6(1.2)$ \\
\hline Intra-abdominal hemorrhage & $1(0.3)$ & $0(0)$ \\
\hline Complication grade $3-5^{\neq}$ & Incidence (\%) & Incidence (\%) \\
\hline Ascites & $9(3.1)$ & $1(0.2)$ \\
\hline Post-resectional liver failure & $10(3.4)$ & $18(3.5)$ \\
\hline Bile leakage & $35(12.0)$ & $23(4.5)$ \\
\hline Intra-abdominal abscess & $30(10.3)$ & $23(4.5)$ \\
\hline Intra-abdominal hemorrhage & $3(1.0)$ & $5(1.0)$ \\
\hline Operative mortality & $10(3.4)$ & $7(1.4)$ \\
\hline Liver surgery specific CEP & $56(19.2)$ & $55(10.7)$ \\
\hline
\end{tabular}

Numbers in parentheses are percentages; ${ }^{*}$ values are mean \pm standard deviation; ${ }^{\dagger}$ resection of three or more liver segments; ${ }^{\ddagger}$ according to the Clavien-Dindo classification ${ }^{9} ; N$, number; CEP, composite endpoint.

patients were included in the present analysis. Their characteristics and postoperative complication rates are depicted in Table 4.2.

At Maastricht University Medical Centre, the rates of the individual components of the proposed liver surgery specific CEP with Clavien-Dindo grade 3 or more within 90 days of initial surgery ranged from 1.0 per cent for intra-abdominal hemorrhage to 12.0 per cent for bile leakage; the mortality rate was 3.4 per cent. The incidence of the liver surgery specific CEP was 19.2 per cent $(n=56)$. The rates of the individual components of the liver surgery specific CEP at the University Hospital of Strasbourg ranged from 0.2 to 4.5 per cent. Fifty-five patients (10.7 per cent) experienced at least one of the six complications of the liver surgery specific CEP (Table 4.2).

Based on the rates of complications in the two data sets, the sample size of a singlecentre RCT intending to show a relative reduction in either the individual complication or the liver surgery specific CEP by one-third, one-half or two-thirds was calculated (Table 4.3). 
Table 4.3 Sample size of each arm of an adequately powered randomized controlled trial aiming to show a one-third, one-half or two-thirds relative reduction of the primary endpoint.

\begin{tabular}{lcccccc}
\hline & \multicolumn{3}{c}{ Maastricht University Medical Centre } & \multicolumn{3}{c}{ University Hospital of Strasbourg } \\
\cline { 2 - 7 } Complication & \multicolumn{3}{c}{ Relative reduction } & \multicolumn{3}{c}{ Relative reduction } \\
\hline Ascites & $\mathbf{3 3 \%}$ & $\mathbf{5 0 \%}$ & $\mathbf{6 6 \%}$ & $\mathbf{3 3 \%}$ & $\mathbf{5 0 \%}$ & $\mathbf{6 6 \%}$ \\
Post-resectional liver failure & 3695 & 1481 & 742 & 58701 & 23484 & 11744 \\
Bile leakage & 3360 & 1348 & 675 & 3261 & 1308 & 656 \\
Intra-abdominal abscess & 1043 & 421 & 212 & 2515 & 1009 & 506 \\
Intra-abdominal hemorrhage & 11661 & 4668 & 2336 & 11661 & 4668 & 2336 \\
Operative mortality & 3360 & 1348 & 675 & 8301 & 3324 & 1664 \\
Liver surgery specific CEP & 514 & 209 & 106 & 1000 & 404 & 203 \\
\hline
\end{tabular}

Assumptions $\alpha=0.05$ and $\beta=0.20$ and two-sided testing; CEP, composite endpoint.

\section{DISCUSSION}

The use of a CEP as primary endpoint of RCTs in liver surgery is not very common at present and uniformity of CEP components is lacking. In this study, a consensus-based and standard liver surgery specific CEP was developed after a systematic literature search and survey among international experts in liver surgery. The proposed liver surgery specific CEP includes ascites, post-resectional liver failure, bile leakage, intra-abdominal hemorrhage, intra-abdominal abscess, and operative mortality, all occurring within 90 days of initial surgery and with Clavien-Dindo severity grade of at least 3 . The event rate of the liver surgery specific CEP was substantially higher than that of its individual components. As a consequence, the theoretical sample size required for an adequately powered RCT using the liver surgery specific CEP as primary endpoint decreased considerably when tested in two patient cohorts from high-volume HPB centres in Europe. Implementation of the liver surgery specific CEP will increase the feasibility of RCTs that use clinical outcomes of liver surgery as primary endpoints.

Investigators in medical disciplines other than liver surgery frequently choose CEPs as the primary endpoint of their RCTs ${ }^{10-12}$. Joint efforts in these fields have already led to the formulation of preferred characteristics of CEP components ${ }^{7,8}$. It has been shown that the use of CEPs can be misleading if components are ill-defined or vary widely in clinical relevance or expected direction of treatment effect ${ }^{6,10,13,14}$. As a consequence, careful selection of CEP components is warranted.

In order to overcome these demerits of CEPs, the aforementioned pitfalls were avoided systematically. First, international consensus on the definitions of complications of liver surgery was reached by a web-based survey. Second, all complications included in the liver surgery specific CEP had to be classified as Clavien-Dindo grade 3 or more because 
this ensures genuine clinical relevance. The Clavien-Dindo classification has been designed to categorize complications of surgery objectively, based on a therapy-oriented severity grade 9 . Although this system basically focuses on a medical perspective, it has recently been proven also to reflect severity from the patients' perspective ${ }^{15}$. Finally, interpretation of the effect size of an intervention and its direction on the individual components is facilitated by adequate reporting of the incidence of all components during the total observation period ${ }^{6,7,10}$. Statistical analysis of these secondary outcomes should be performed using adequate consideration of a spending and the question of multiple testing ${ }^{3,16}$. It is important to appreciate that secondary outcomes, even if nominally significant, are of secondary importance and not decisive in favour of one treatment if the primary endpoint fails to show a significant effect ${ }^{4}$.

Only eight of 47 RCTs used a CEP as primary endpoint, of which four defined the CEP as "postoperative complications". The use of postoperative complications as CEP is confusing as it is unclear which individual complications account for its incidence. It has been proven that clinically irrelevant complications can contribute greatly to the event rate of such CEPs ${ }^{10}$. Therefore, explicit statement of CEP components and assurance of their clinical relevance are essential.

The effect of introducing a liver surgery specific CEP was assessed using two patient cohorts. The complication rates in both cohorts were within the 95 per cent prediction intervals that were calculated in a recent meta-analysis on complications of liver surgery ${ }^{2}$. Therefore, these two cohorts were considered representative of current HPB practice. The incidence of the liver surgery specific CEP was 10.7 per cent in the Strasbourg and 19.2 per cent in the Maastricht cohort. The difference between the two data sets might be explained by differences in surgical technique and perioperative care or, alternatively, by differences in data registration and definitions of complications. Adoption of standard registration methods and uniform definitions of complications of liver surgery can decrease the latter type of heterogeneity. The American College of Surgeons National Surgical Quality Improvement Programme could serve as an example of standardized reporting of outcomes of HPB surgery (www. acsnsqip.org) ${ }^{17}$.

A drawback of this study is the inevitable selection bias introduced by the application of a survey on invitation. HPB surgeons from all continents were invited to complete the survey. The majority of responses came from Europe, and therefore, the results of the survey represent the current view on trial design in the European HPB community. An open survey is currently under development in order to collect a broader opinion of the HPB community on definitions and components of the liver surgery specific CEP. As it might prove difficult to use the liver surgery specific CEP as primary endpoint for all RCTs in liver surgery, it may be necessary to design CEPs customized for trials focussing on interventions with medication or surgical technique. Furthermore, assigning weights to components based on their clinical impact or expression of the liver surgery specific CEP 
on an ordinal six-point scale may be considered in the future. However, as the majority of patients in both institutes had an uneventful clinical course, only marginal gain of power should be expected from the latter.

Prospective validation of the proposed liver surgery specific CEP, using data from institutes originating from all continents collected by well-trained research assistants using uniform definitions, is warranted. 


\section{REFERENCES}

1. Kaido T. Analysis of randomized controlled trials on hepatopancreatic surgery. Dig Dis Sci 2006; 51: 1761-6.

2. van den Broek MA, van Dam RM, Malago M, Dejong CH, van Breukelen GJ, Olde Damink SW. Feasibility of randomized controlled trials in liver surgery using surgery-related mortality or morbidity as endpoint. Br J Surg 2009; 96: 1005-1014.

3. Freemantle N. Interpreting the results of secondary end points and subgroup analyses in clinical trials: should we lock the crazy aunt in the attic? Bmj 2001; 322: 989-91.

4. Freemantle N. How well does the evidence on pioglitazone back up researchers' claims for a reduction in macrovascular events? Bmj 2005; 331: 836-8.

5. Washburn K. Endpoints in trials for clinical liver transplantation. Curr Opin Organ Transplant 2008; 13: 252-6.

6. Freemantle N, Calvert M, Wood J, Eastaugh J, Griffin C. Composite outcomes in randomized trials: greater precision but with greater uncertainty? Jama 2003; 289: 2554-9.

7. Ferreira-Gonzalez I, Permanyer-Miralda G, Busse JW, Bryant DM, Montori VM, Alonso-Coello P, et al. Methodologic discussions for using and interpreting composite endpoints are limited, but still identify major concerns. J Clin Epidemiol 2007; 60: 651-7.

8. Neaton JD, Gray G, Zuckerman BD, Konstam MA. Key issues in end point selection for heart failure trials: composite end points. J Card Fail 2005; 11: 567-75.

9. Dindo D, Demartines N, Clavien PA. Classification of surgical complications: a new proposal with evaluation in a cohort of 6336 patients and results of a survey. Ann Surg 2004; 240: 205-13.

10. Ferreira-Gonzalez I, Busse JW, Heels-Ansdell D, Montori VM, AkI EA, Bryant DM, et al. Problems with use of composite end points in cardiovascular trials: systematic review of randomised controlled trials. Bmj 2007; 334: 786-92.

11. Califf RM, Harrelson-Woodlief $L$, Topol EJ. Left ventricular ejection fraction may not be useful as an end point of thrombolytic therapy comparative trials. Circulation 1990; 82: 1847-53.

12. Lim E, Brown A, Helmy A, Mussa S, Altman DG. Composite outcomes in cardiovascular research: a survey of randomized trials. Ann Intern Med 2008; 149: 612-7.

13. Bethel MA, Holman R, Haffner SM, Califf RM, Huntsman-Labed A, Hua TA, et al. Determining the most appropriate components for a composite clinical trial outcome. Am Heart J 2008; 156: 63340.

14. Montori VM, Permanyer-Miralda G, Ferreira-Gonzalez I, Busse JW, Pacheco-Huergo V, Bryant D, et al. Validity of composite end points in clinical trials. Bmj 2005; 330: 594-6.

15. Clavien PA, Barkun J, de Oliveira ML, Vauthey JN, Dindo D, Schulick RD, et al. The Clavien-Dindo classification of surgical complications: five-year experience. Ann Surg 2009; 250: 187-96.

16. Neuhauser M. How to deal with multiple endpoints in clinical trials. Fundam Clin Pharmacol 2006; 20: 515-23.

17. Pitt HA, Kilbane M, Strasberg SM, Pawlik TM, Dixon E, Zyromski NJ, et al. ACS-NSQIP has the potential to create an HPB-NSQIP option. HPB (Oxford) 2009; 11: 405-13. 



\section{Part II}

Assessing outcomes of liver surgery:

hepatic damage as a result of surgical technique 



\section{Chapter 5}

\section{Liver manipulation during liver surgery in humans is associated with hepatocellular damage and hepatic inflammation}

Published as:

van den Broek MA, Shiri-Sverdlov R, Schreurs JW, Bloemen JG, Bieghs V, Rensen SS, Dejong $\mathrm{CH}$, Olde Damink SW. Liver manipulation during liver surgery in humans is associated with hepatocellular damage and hepatic inflammation. Liver Int 2013; 


\section{ABSTRACT}

\section{Background}

Manipulation of the liver during liver surgery results in profound hepatocellular damage. Experimental data show that mobilization-induced hepatocellular damage is related to hepatic inflammation. To date, information on this link in humans is lacking. As it is possible to modulate inflammation, it is clinically relevant to unravel this relation. This observational study aimed to establish the association between liver mobilization and hepatic inflammation in humans.

\section{Methods}

Consecutive patients requiring mobilization of the right hemi-liver during liver surgery were studied. Plasma samples and liver biopsies were collected prior to and directly after mobilization and after transection of the liver. Hepatocellular damage was assayed by liver fatty acid-binding protein (L-FABP) and aminotransferase levels. Hepatic inflammation was determined by (a) immunohistochemical identification of myeloperoxidase (MPO) and CD68- positive cells and (b) hepatic gene expression of inflammatory and cell adhesion molecules (IL-1 $\beta$, IL-6, IL-8, VCAM-1 and ICAM-1).

\section{Results}

A total of 25 patients were included. L-FABP levels increased significantly during mobilization ( $301 \pm 94 \mathrm{ng} / \mathrm{mL}$ to $1599 \pm 362 \mathrm{ng} / \mathrm{mL}, \mathrm{p}=0.008$ ), as did ALAT levels ( $36 \pm 5 \mathrm{IU} / \mathrm{L}$ to $167 \pm 21 \mathrm{IU} / \mathrm{L}, \mathrm{p}<0.001)$. A significant increase in MPO $(\mathrm{p}=0.001)$ and CD68 $(p=0.002)$ positive cells was noticed in the liver after mobilization. The number of MPO-positive cells correlated with the duration of mobilization (Pearson correlation $=0.505, p=0.033$ ). Hepatic gene expression of pro-inflammatory cytokines IL-1 $\beta$ and IL-6, chemo-attractant IL-8 and adhesion molecule ICAM-1 increased significantly during liver manipulation.

\section{Conclusion}

Liver mobilization is associated with hepatocellular damage and liver inflammation, as shown by infiltration of inflammatory cells and upregulation of genes involved in acute inflammation. 


\section{INTRODUCTION}

Surgical resection is the ultimate treatment for a variety of benign and malignant liver tumours. During liver surgery, there is a delicate balance between the attempt to achieve surgery with curative intent and the necessity to leave adequate remnant liver volume in order to avoid post-resectional liver failure (PLF). Risk factors for the development of PLF may either be surgery- or patient-related'.

With respect to surgery-related risk factors, excessive intra-operative blood loss and the need for blood transfusion are associated with adverse post-resectional outcomes ${ }^{2}$. In order to limit blood loss during liver surgery, different surgical techniques have been introduced. One of these techniques is mobilization of the liver prior to transection. During mobilization, the liver is forcefully manipulated in order to dissect its ligaments and control direct venous branches to the inferior caval vein. Recent data deliver convincing evidence that mobilization of the liver in itself causes substantial hepatocellular injury ${ }^{3-7}$. The highly sensitive liver cell damage markers liver fatty acid-binding protein (L-FABP), arginase-1, glutathione-s-transferase- $a$ and cell-free circulating albumin-mRNA increased significantly during mobilization of the liver and did not increase thereafter during either inflow occlusion or transection ${ }^{3-7}$. Of important notice, manipulation-induced liver cell damage negatively affected postoperative outcomes in patients undergoing liver surgery for hepatocellular carcinoma ${ }^{7}$.

The pathogenesis of mobilization-induced liver damage has been studied in detail in a murine model of liver transplantation ${ }^{8-10}$. In short, mobilization of the liver induced neuronal mediated disturbances in the hepatic microcirculation leading to both liver cell damage and hepatic inflammation. Activated Kupffer cells seemed to play a central role as modulation of their function largely prevented hepatocellular damage and improved experimental outcome ${ }^{11}$. In man, it has been shown that systemic inflammation, reflected by plasma interleukin-6 (IL-6) levels, followed liver manipulation during surgery ${ }^{3}$. However, the source of this systemic inflammatory response is yet unidentified in humans. Based on aforementioned experimental observations, hepatic inflammation might well be involved in the cascade of manipulation-induced liver cell damage and systemic inflammation.

Given the possibility to modulate the inflammatory response, identification of the link between manipulation-induced liver cell injury and inflammation in man could identify novel therapeutic strategies for its prevention. This study aimed to establish the association between liver mobilization, hepatocellular damage and hepatic inflammation in patients undergoing liver surgery. 


\section{METHODS}

\section{Patients}

Consecutive patients scheduled to undergo liver surgery requiring full mobilization of the right hemi-liver at Maastricht University Medical Centre between October 2007 and June 2009 were included in this observational study. Exclusion criteria were (a) the presence of cirrhosis of the liver confirmed by preoperative liver biopsy, (b) repeat liver surgery, (c) laparoscopic liver surgery, (d) use of anti-inflammatory drugs, (e) presence of renal failure (defined as serum creatinine above $137 \mu \mathrm{mol} / \mathrm{L}$ in males and above 104 $\mu \mathrm{mol} / \mathrm{L}$ in females ${ }^{12}$ ), (f) performance of an extra-hepatic procedure, and (g) participation in another trial.

Resections were divided into major (3 or more Couinaud segments) or minor (less than 3 Couinaud segments or non-anatomical wedge resections) $)^{13}$. All data were prospectively entered in a database and the clinical course of the participants was studied up until 90 days after discharge. The incidence rate of the liver surgery specific composite endpoint and its individual components (ascites, bile leakage, intra-abdominal hemorrhage, intra-abdominal abscess, PLF and operative mortality) was calculated ${ }^{14}$. The study was approved by the Medical Ethics Committee of Maastricht University Medical Centre and all participating individuals gave written informed consent.

\section{Surgical procedure}

Patients routinely had two peripheral venous catheters and indwelling catheters in a jugular vein and radial artery. Immediately preoperatively, all patients received a single intravenous dose of 2200 mg amoxicillin/clavulanic acid as antibiotic prophylaxis. Propofol and isoflurane were used as anaesthetics. Surgical procedures were commenced using a subcostal bilateral incision as described earlier ${ }^{5}$. An Omni-Flex General Retractor System (Integra LifeSciences Corporation, Plainsboro, NJ, USA) was used to improve exposure. After dissection of the teres hepatis ligament, the procedure was continued with dissection of the falciform ligament and further mobilization of the right hemi-liver from the posterior abdominal wall. Thereafter, the liver was rotated anteriorly and to the left in order to dissect direct venous branches to the inferior caval vein. Full mobilization was reached when the caval vein was dissected free of all its attachments at the 12 o'clock anterior surface. Subsequently, an intra-operative ultrasound was performed which directed the surgical strategy. A Cavitron Ultrasonic Surgical Aspirator (CUSA system 200 macrodissector, Cavitron Surgical Systems, Stamford, CT, USA) and Argon beam coagulation (Force GSU System, Valleylab, Boulder, CO, USA) were used for liver transection. Inflow occlusion was not routinely applied. If necessary, a complete or selective Pringle maneuver (with $15 \mathrm{~min}$ or $30 \mathrm{~min}$ ischemic cycles) or ligation of the appropriate portal pedicle vessels was applied ${ }^{5}$. During transection, central venous pressure was 
maintained below $5 \mathrm{mmHg}$. Postoperative care was provided according to an enhanced recovery after liver surgery programme ${ }^{15}$.

\section{Blood and tissue sampling}

Before, during and after the operative procedure, arterial blood was drawn from the radial artery catheter according to a predetermined protocol at different time points (Figure 5.1). Blood samples were transferred to prechilled EDTA tubes and subsequently centrifuged at $4^{\circ} \mathrm{C}$ at $3500 \mathrm{xg}$ for $15 \mathrm{~min}$. Plasma was stored at $-80^{\circ} \mathrm{C}$ until batch analysis.

Liver wedge biopsies were taken using scissors at fixed time points during the procedure from segment 5 of the liver. The first liver wedge biopsy was obtained immediately after opening of the abdomen and before touching or manipulating the intestines or liver, the second biopsy was collected after full mobilization of the right hemi-liver and before application of inflow occlusion or liver transection, and the third after liver transection. Defined $0.5 \times 0.5 \mathrm{~cm}$ fragments of liver tissue were cut, snap-frozen in liquid nitrogen and stored at $-80^{\circ} \mathrm{C}$. Fragments of the same size were immersed in Tissue-Tek optimal cutting temperature compound (Sakura Finetek Europe, Zoeterwoude, The Netherlands) and mounted on a piece of cork before they were frozen in prechilled isopentane on dry ice and stored at $-80^{\circ} \mathrm{C}$.

\section{Hepatocellular damage}

The extent of hepatocellular damage was assessed by plasma concentrations of L-FABP and aminotransferases. L-FABP is a sensitive marker for the detection of liver cell damage $^{16,17}$. L-FABP levels were determined using a commercially available ELISA (Hycult Biotechnology, Uden, the Netherlands). According to the manufacturer's manual, L-FABP plasma levels in healthy individuals were approximately $12 \mathrm{ng} / \mathrm{mL}$. Alanine aminotransferase (ALAT) levels were assayed by the clinical chemistry laboratory of Maastricht University Medical Centre. The upper limit of normal was $35 \mathrm{IU} / \mathrm{L}$.

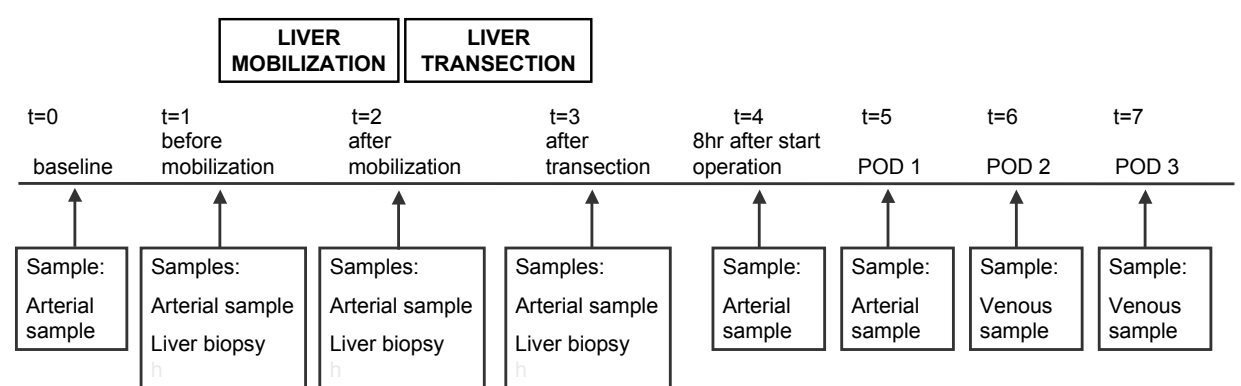

Figure 5.1 Flowchart of blood and tissue collection. Legend: POD, postoperative day. 


\section{Immunohistochemistry of the liver}

Tissue-Tek embedded frozen liver biopsies were cut in $7 \mu \mathrm{m}$ sections, fixed in acetone for $10 \mathrm{~min}$ and subsequently blocked for endogenous peroxidase activity by incubation in 0.3 per cent $\mathrm{H}_{2} \mathrm{O}_{2}$ in PBS. Primary antibodies were applied for $1 \mathrm{hr}$ after blocking with 10 per cent serum. The following primary antibodies were used: (i) rabbit anti-human myeloperoxidase (MPO) antiserum (dilution 1:1000; Dako, Glostrup, Denmark) as marker for neutrophils and macrophages; (ii) anti-CD68 (clone Kp1, dilution 1:400; Dako, Glostrup, Denmark) specific for monocytes/macrophages; and (iii) anti-caspase-3-mediated cleavage generated neo-epitope of cytokeratin 18 (M30, dilution 1:50; Roche, Mannheim, Germany) specific for hepatocyte apoptosis, as described previously ${ }^{18}$. Secondary antibodies consisted of horseradish peroxidase-labeled goat-anti-rabbit lgG (1:500; Jackson immunoResearch, Suffolk, UK) for MPO-staining. For CD68 and M30-staining, biotinylated rabbit-anti-mouse IgG was applied as secondary antibody (1:300 and 1:500 dilution, respectively), and the StrepAB/HRP complex (Dako, Glostrup, Denmark) was used for signal enhancement. Staining was visualized by DAB followed by haematoxylin for nuclear counterstaining. The stained slides were photographed at 200x magnification using a Nikon digital camera DXM1200 and ACT-1 v2.63 software from Nikon Corporation. Cells were counted in 6 randomly selected microscopical views, and cell numbers were noted as cells $/ \mathrm{mm}^{2}$ for MPO and M30-staining and as 0-3 ordinal scale for CD68staining. The number of CD68-positive cells was categorized as follows: 0 (none), 1 (few), 2 (moderate numbers), and 3 (many). In addition, the morphology of CD68-positive cells was graded on a 1-3 scale as follows: 1 (normal appearance), 2 (moderate enlargement), and 3 (substantial enlargement).

\section{Gene expression of inflammatory mediators in the liver}

Expression of genes encoding for inflammatory mediators and cell adhesion molecules was determined in liver biopsies taken at three time points during surgery (Figure 5.1). Genes of interest included interleukin 1 beta (IL-1 $\beta$ ) and interleukin 6 (IL-6), both pro-inflammatory cytokines involved in macrophage activation, interleukin 8 (IL-8), a chemokine involved in recruitment of inflammatory cells, and vascular cell adhesion molecule (VCAM-1) and intercellular adhesion molecule (ICAM-1), important for adhesion and migration of inflammatory cells.

Hepatic gene expression was assessed by real-time quantitative polymerase chain reaction (PCR). Total RNA was isolated from snap-frozen liver samples by using Tri-reagent (Sigma-Aldrich, St. Louis, MO, USA). Reverse transcription was performed using the iScript cDNA synthesis kit (Bio-Rad, Hercules, CA, USA). Real-time PCR was performed on a Bio-Rad MyIQ using IQ SYBR Green Supermix (Bio-rad, Hercules, CA, USA). Primers for target genes were developed using Primer Express version 2.0 (Applied Biosystems, Foster City, CA, USA). Sequences of the applied PCR primers are listed in Appendix 9. To 
standardize for cDNA concentration in the samples, the housekeeping gene cyclophylin A (peptidylprolyl isomerase A) was used. For calculations of the initial amount of mRNA present in the sample, the relative standard curve method was used.

\section{Statistical analysis}

Data are given as mean and standard error of the mean or median with range, depending on the nature of the data. Differences in hepatocellular damage markers, number of MPO and M30-positive cells and hepatic gene expression between the three time points during liver surgery (before mobilization, after mobilization and after transection) were calculated using the paired sample t-test. For CD68 number and morphology, median values were compared using Wilcoxon's signed rank test. In addition, correlations between duration of mobilization, influx of inflammatory cells and hepatic gene expression were calculated. A p-value below 0.050 was considered statistically significant. Statistical analysis was performed using SPSS version 20 for Windows (SPSS Inc., Chicago, IL, USA).

\section{RESULTS}

\section{Patient flow}

Between October 2007 and July 2009, one-hundred and two patients were scheduled to undergo a partial hepatic resection. Of these, 25 patients fulfilled the inclusion criteria. Reasons for exclusion were: presence of underlying liver disease confirmed by preoperative liver biopsy $(n=4)$, repeat liver surgery $(n=6)$, laparoscopic liver surgery $(n=7)$, no informed consent $(n=9)$, participation in another trial $(n=6)$, no liver resection during surgery $(n=15)$, no formal mobilization of right hemi-liver $(n=24)$, and performance of an extra-hepatic procedure $(n=6)$.

\section{Patient characteristics}

Characteristics of the included patients are shown in Table 5.1. Liver surgery was performed because of benign hepatic disease in 1 and secondary hepatic malignancies in 24 patients, consisting of colorectal liver metastases in 23 and carcinoid metastases in 1 patient, respectively. Major liver resections were performed in 17 and minor in 8 patients, with a median number of 4 resected segments (range 1-4). Mean operative time was $213 \pm 11 \mathrm{~min}$, of which $65 \pm 5 \mathrm{~min}$ were used for mobilization of the right hemiliver and $94 \pm 8 \mathrm{~min}$ for transection of liver parenchyma. The extent of resection did not influence the duration of liver mobilization $(70 \pm 6 \mathrm{~min}$ for major resections versus $53 \pm 8$ min for minor resections, $\mathrm{p}=0.112$ ). During transection, a Pringle maneuver was applied in 15 patients (60 per cent). Mean cumulative ischemia time in patients undergoing tran- 
Table 5.1 Patient characteristics.

\begin{tabular}{|c|c|}
\hline & $N=\mathbf{2 5}$ \\
\hline \multicolumn{2}{|l|}{ Baseline characteristics } \\
\hline Age (years) & $61 \pm 2$ \\
\hline Sex (male) & $17(68)$ \\
\hline BMI $\left(\mathrm{kg} / \mathrm{m}^{2}\right)$ & $26 \pm 1$ \\
\hline \multicolumn{2}{|l|}{ Primary disease } \\
\hline Benign & $1(4)$ \\
\hline Malignant & $24(96)$ \\
\hline \multicolumn{2}{|l|}{ Preoperative laboratory tests } \\
\hline ALAT (IU/L) & $30 \pm 4$ \\
\hline Bilirubin (total) ( $\mu \mathrm{mol} / \mathrm{L})$ & $13 \pm 1$ \\
\hline Prothrombin time (sec) & $11 \pm 1$ \\
\hline Creatinine $(\mu \mathrm{mol} / \mathrm{L})$ & $87 \pm 3$ \\
\hline \multicolumn{2}{|l|}{ Operative variables } \\
\hline \multicolumn{2}{|l|}{ Type of resection } \\
\hline Right hepatectomy & $12(48)$ \\
\hline Trisectionectomy & $4(16)$ \\
\hline Segmentectomy & $9(36)$ \\
\hline Median no. resected segments (range) & $4(1-4)$ \\
\hline Duration of surgery (min) & $213 \pm 11$ \\
\hline Mobilization time (min) & $65 \pm 5$ \\
\hline Transection time (min) & $94 \pm 8$ \\
\hline Pringle maneuver & $15(60)$ \\
\hline Selective Pringle & $7(28)$ \\
\hline Complete Pringle & $8(32)$ \\
\hline Total blood loss (mL) & $1002 \pm 167$ \\
\hline \multicolumn{2}{|l|}{ Postoperative outcome } \\
\hline Liver surgery specific composite endpoint & $4(16)$ \\
\hline
\end{tabular}

Numbers indicate mean \pm standard error or absolute number (percentage) unless otherwise indicated; $N$, number; BMI, body mass index; ALAT, alanine aminotransferase.

section with a complete Pringle maneuver was $55 \pm 7 \mathrm{~min}$. For patients with a selective Pringle maneuver of the right hemi-liver, mean cumulative ischemia time was $30 \pm 4 \mathrm{~min}$.

The incidence of the liver surgery specific composite endpoint was 16 per cent (4 out of 25 patients). The component accounting for this incidence was bile leakage in all 4 patients. The rates of PLF and operative mortality were zero.

\section{Liver cell damage markers increase significantly after liver mobilization}

To characterize liver cell damage secondary to liver manipulation, L-FABP and ALAT levels were measured. Mean arterial L-FABP levels increased significantly during mobilization 
of the right hemi-liver (from $301 \pm 94 \mathrm{ng} / \mathrm{mL}$ to $1599 \pm 362 \mathrm{ng} / \mathrm{mL}, \mathrm{p}=0.008$ ) and did not increase significantly thereafter $(2791 \pm 872 \mathrm{ng} / \mathrm{mL}, \mathrm{p}=0.696$ versus after mobilization), as depicted in Figure 5.2A. ALAT concentration also increased significantly during mobilization of the right hemi-liver (from $36 \pm 5 \mathrm{IU} / \mathrm{L}$ to $167 \pm 21 \mathrm{IU} / \mathrm{L}, \mathrm{p}<0.001$ ) and further increased during transection $(408 \pm 61 \mathrm{IU} / \mathrm{L}, \mathrm{p}<0.001$ versus after mobilization, Figure $5.2 \mathrm{~B})$. The increase in hepatocellular damage markers after mobilization did not relate to the extent of hepatic resection or the duration of mobilization (data not shown).

\section{Liver mobilization results in hepatocyte apoptosis}

Staining for M30 indicated that hepatocyte apoptosis tended to increase after mobilization ( $p=0.090$ ) and returned to baseline after transection (Figure 5.3). There was a significant correlation between the duration of mobilization and the absolute increase in M30-positive cells (Pearson correlation $=0.507, \mathrm{p}=0.027$ ).

\section{Liver mobilization increases the number of hepatic immune cells}

To study mobilization-mediated inflammation, inflammatory cells were identified in liver biopsies at 3 time points in 22 of the 25 included patients ( 88 per cent) by detection of MPO and CD68. Staining for MPO, a marker for neutrophils and macrophages, revealed a significant increase in absolute number of MPO-stained cells in liver tissue after mobi-

A

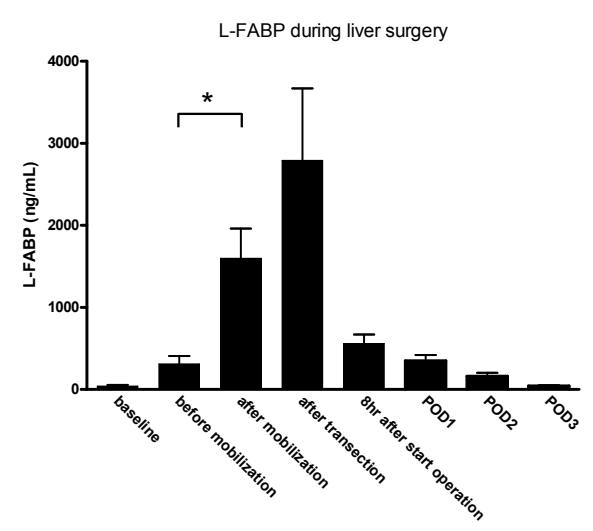

B

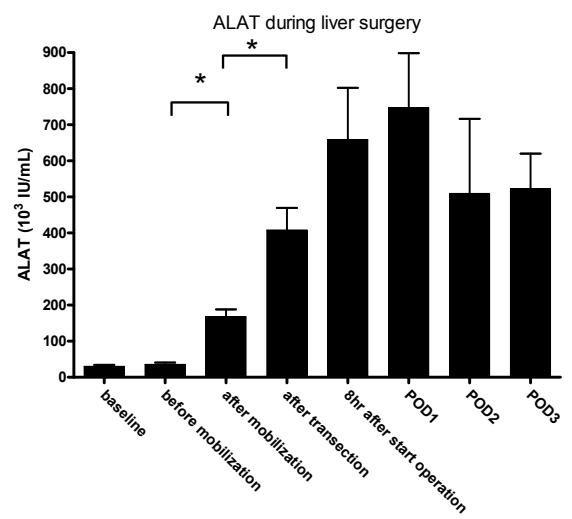

Figure 5.2A and 5.2B Course of hepatocellular damage markers L-FABP and ALAT during and after liver surgery.

(A) L-FABP levels increased significantly during mobilization of the liver. L-FABP levels peaked at the end of surgery and decreased thereafter (mean and standard error). (B) ALAT levels increased during mobilization of the liver and continued to increase significantly thereafter, until reaching their peak at the first postoperative day (mean and standard error).

Legend: L-FABP, liver fatty acid-binding protein; ALAT, alanine aminotransferase; POD, postoperative day; * indicates $p<0.050$. 


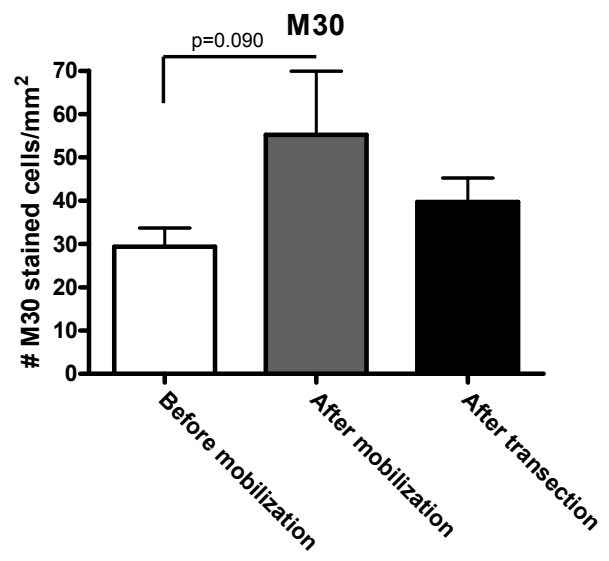

Figure 5.3 Apoptosis of hepatocytes during liver surgery as evidenced by M30-staining. Liver mobilization resulted in a non-significant twofold increase in M30-stained cells (mean and standard error).

lization ( $p=0.001)$, which did not rise significantly during transection $(p=0.080)$ (Figure 5.4A). There was a significant correlation between the absolute increase of MPO-positive cells after mobilization and the duration of mobilization (Pearson correlation $=0.505$, $\mathrm{p}=0.033)$.

Staining for CD68, a specific marker for macrophages, showed a small but significant increase in CD68-positive cells after mobilization (median score 2 [1-2.5] before versus 2.5 [2-3] after mobilization, $\mathrm{p}=0.002$ ) (Figure 5.4B). These CD68-positive cells had a different morphology, characterized by enlargement and rounding after mobilization (median morphology score 1.5 [1-3] before versus 2 [1-3] after mobilization, $p=0.003$ ) (Figure 5.4C), suggesting that these cells represented monocytes that infiltrated the liver.

\section{Liver mobilization induces hepatic expression of inflammatory genes}

Enhanced expression of genes of pro-inflammatory cytokines, chemokines and adhesion molecules plays a significant role in promoting immune cell infiltration. In agreement with the histological findings, hepatic mRNA levels of IL-1 $\beta$, IL- 6 and IL-8 (Figure $5.5 \mathrm{~A}-\mathrm{C}$ ) significantly increased after mobilization compared with baseline levels. Rise in expression ranged from 23 -fold for IL-1 $\beta, 65$-fold for IL-8 and 137 -fold for IL-6. The expression of the chemokine IL-8 significantly correlated with the absolute increase in MPO-positive cells (Pearson correlation $=0.516, p=0.049$ ). After transection, the mRNA levels of IL-1 $\beta$, IL- 6 and IL-8 further increased.

The expression of the cell adhesion molecule ICAM-1, but not VCAM-1, increased significantly after mobilization (Figure 5.5D-E). The increase in ICAM-1 gene expression 

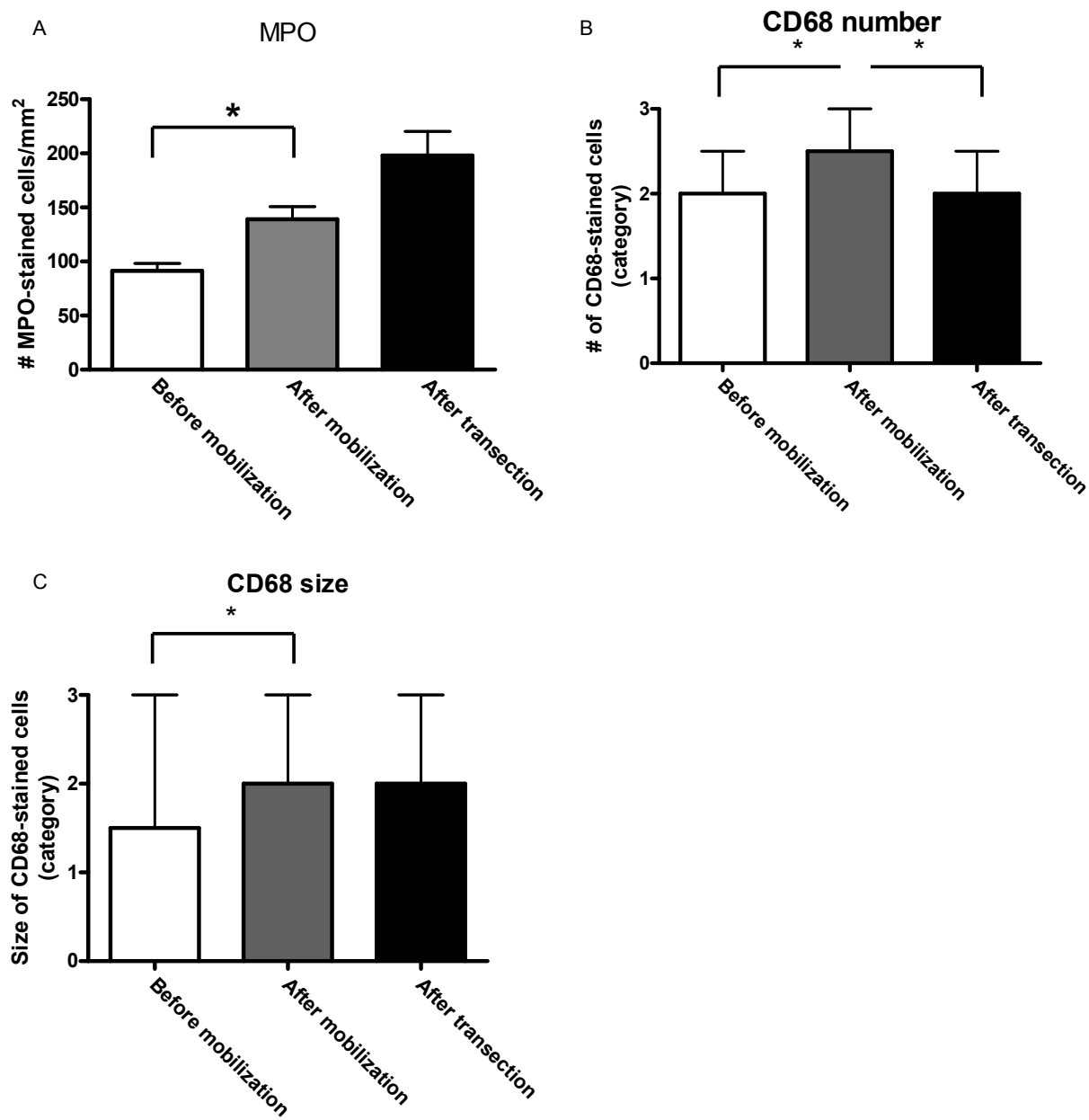

Figure 5.4A-C MPO and CD68-positive immune cells during liver surgery.

(A) Number of MPO-stained cells increased significantly during manipulation (mean and standard error). (B) CD68-staining revealed an increased number of CD68-positive cells in the liver after mobilization (median and range). (C) CD68-positive cells increased in size after mobilization of the liver (median and range). Legend: MPO, myeloperoxidase; CD68, cluster of differentiation 68; * indicates $p<0.050$.

tended to correlate with the absolute increase in MPO-positive cells (Pearson correlation $=0.455, p=0.089$ ).

\section{DISCUSSION}

The present study was designed to establish the association between liver mobilization, hepatocellular damage and hepatic inflammation during liver surgery in humans. Our data corroborate earlier observations that liver mobilization induces profound liver cell 
A
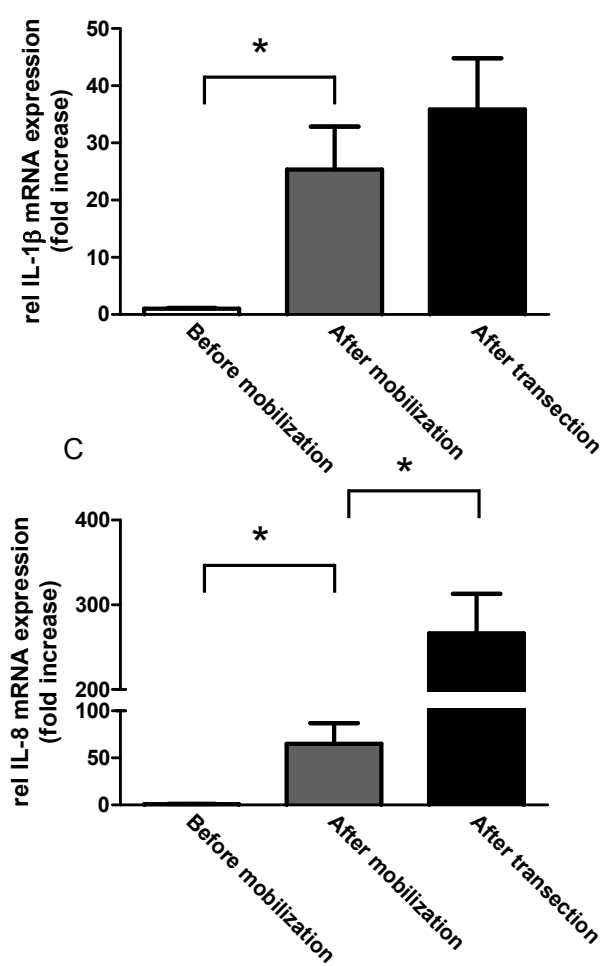

$\mathrm{E}$

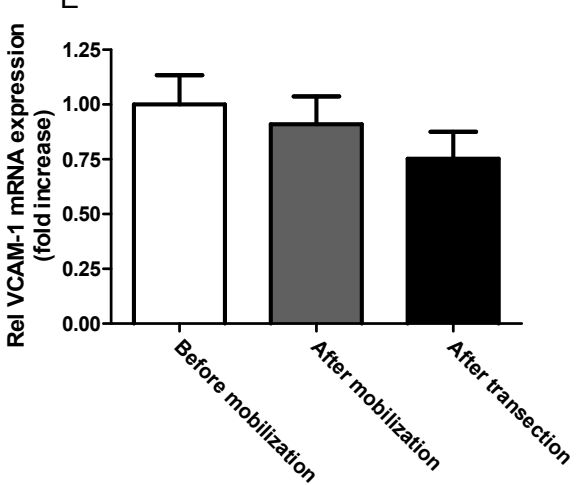

B
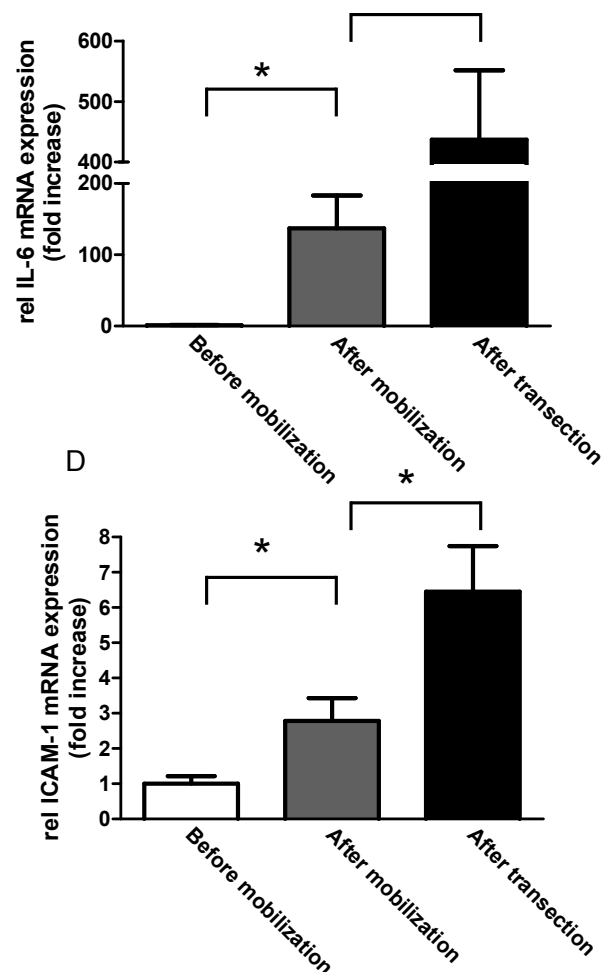

Figure 5.5A-E Relative hepatic gene expression of inflammatory cytokines and cell adhesion molecules during liver surgery.

(A-C) Relative expression of IL-1 $\beta$, IL-6 and IL-8 significantly increased during liver surgery (mean and standard error). (D-E) Relative expression of ICAM-1 increased, whereas relative expression of VCAM-1 remained fairly constant (mean and standard error).

Legend: IL, interleukin; VCAM, vascular cell adhesion molecule; ICAM, intercellular adhesion molecule; * indicates $p<0.050$. 
damage, as evidenced by an early and significant rise in the hepatocellular damage markers L-FABP and ALAT. In addition, we present novel, human data indicating that liver mobilization is associated with liver cell apoptosis and hepatic inflammation, as shown by an increase in MPO and CD68-positive inflammatory cells and upregulation of mRNA of pro-inflammatory cytokines in the liver. The extent of apoptosis and increase in inflammatory cells was significantly related to the duration of mobilization.

Manipulation of the liver immediately led to hepatocellular damage. Levels of the damage markers L-FABP and ALAT were significantly increased after mobilization and there was a trend towards increased hepatocyte apoptosis, evidenced by M30-positive hepatocytes. We previously showed that the increase in systemic plasma levels of L-FABP and ALAT after liver mobilization solely resulted from hepatic release and not from the hepatotoxic effects of anesthesia or surgical trauma of performing a laparotomy ${ }^{3,5}$. Interestingly, the course of ALAT levels showed a different pattern compared to L-FABP levels. ALAT levels peaked on the first postoperative day, at the same time and in the same range as reported by other groups ${ }^{19}, 20$, whereas L-FABP levels reached their maximum values at the end of surgery and rapidly decreased thereafter. This might be a reflection of the fact that L-FABP is a more direct and sensitive marker for the detection of liver cell damage as compared to ALAT, due to the small molecular mass and short half-life of L-FABP $16,17,21$.

Inflammation has previously been recognized as an important element in the multifaceted process leading to manipulation-induced tissue injury in rodents ${ }^{8,22}$. In man, however, the relation between liver manipulation, hepatocellular damage and hepatic inflammation was unknown. Here, we provide the first data in man showing that liver mobilization is accompanied by hepatocyte apoptosis and influx of inflammatory cells in hepatic tissue. Additional CD68-staining identified involvement of monocytes and macrophages. It has previously been shown that macrophages such as Kupffer cells are able to express MPO, which might reflect their pro-inflammatory status ${ }^{18,23}$. The present findings of enhanced hepatic expression of the typical pro-inflammatory macrophage markers IL-1 $\beta$ and IL- 6 are in line with this hypothesis.

It might be clinically relevant to prevent mobilization-induced liver cell damage, hepatocyte apoptosis and inflammation by either employment of alternative surgical techniques or modulation of inflammation. With respect to the latter, it remains unclear whether the presence and activation of inflammatory cells secondary to liver manipulation is beneficial or not. In general, unrestrained activation of inflammatory cells following trauma is believed to exacerbate damage, although initially intended to maintain homeostasis ${ }^{24,25}$. As human evidence on the clinical consequences of mobilization-induced liver inflammation is lacking to date, assumptions are solely based on results of animal studies. In animal models of liver manipulation-induced hepatocellular damage, the administration of gadolinium chloride, a Kupffer cell toxicant, or glycine, which prevents 
Kupffer cell activation, led to decreased hepatocellular damage and improved survival after liver transplantation ${ }^{8,26}$. In other areas of research, modulation of inflammation secondary to manipulation has proven to be beneficial in terms of clinical outcomes in animals as well as humans ${ }^{27-29}$. Intervention studies with anti-inflammatory drugs, aiming at a modulation of monocyte influx or macrophage activity, may be performed to elucidate whether a dampened inflammatory response would lead to less tissue injury and, more importantly, improved clinical outcome in patients undergoing liver surgery.

Alternative surgical techniques that require less manipulation of the liver, are already available, such as laparoscopic liver resection or liver resection using the anterior approach $^{30,31}$. Laparoscopy might be advantageous, although several reports show an unfavorable effect of the pneumoperitoneum on hepatic microcirculation ${ }^{32,}{ }^{33}$. This is undesirable as animal study data suggest that microcirculatory failure mediates manipulation-induced liver cell damage ${ }^{8}$. Liver resection using the anterior approach involves initial completion of parenchymal transection without mobilization of the right hemi-liver ${ }^{30,34}$. Advantages include minimal interruption of hepatic circulation during surgery, improved liver function and reduced risk of spilling viable cancer cells into the circulation, at the cost of an enhanced risk of bleeding ${ }^{30}$. Indeed, Liu and coworkers showed reduced cell-free circulating albumin-mRNA levels, as a marker of circulating liver cells, and a lower incidence of PLF in patients undergoing liver resection using the anterior approach compared with the conventional approach ${ }^{7}$. Comparison of hepatocellular damage, hepatic inflammation and clinical outcomes between patients undergoing liver surgery using the conventional approach versus the anterior approach is warranted.

The trigger for immune activation in mobilization-induced liver damage in man remains to be identified. Oxidative stress-related danger signals, resulting from microcirculatory failure, might well be involved as triggers of local inflammation ${ }^{11}$. Livers with reduced anti-oxidant capacity and pre-existent microvascular damage, such as livers suffering from chemotherapy-associated hepatotoxicity, may therefore be at additional risk $^{35,36}$. Moreover, the relation between mobilization-induced hepatocellular damage, hepatic inflammation and clinical outcome remains to be established in a trial using larger patient groups ${ }^{37}$.

Taken together, the results of the present study provide evidence of an association between liver mobilization, hepatocellular damage and hepatic inflammation in man, in line with previous results from animal studies. They form the basis for the development of novel therapies to prevent mobilization-induced damage early during liver surgery, such as the administration of immune-modulating drugs or adoption of alternative surgical techniques. 


\section{REFERENCES}

1. van den Broek MA, Olde Damink SW, Dejong CH, Lang H, Malago M, Jalan R, et al. Liver failure after partial hepatic resection: definition, pathophysiology, risk factors and treatment. Liver Int 2008; 28: 767-80.

2. Kooby DA, Stockman J, Ben-Porat L, Gonen M, Jarnagin WR, Dematteo RP, et al. Influence of transfusions on perioperative and long-term outcome in patients following hepatic resection for colorectal metastases. Ann Surg 2003; 237: 860-9.

3. van de Poll MC, Derikx JP, Buurman WA, Peters WH, Roelofs HM, Wigmore SJ, et al. Liver manipulation causes hepatocyte injury and precedes systemic inflammation in patients undergoing liver resection. World J Surg 2007; 31: 2033-8.

4. Chouker A, Martignoni A, Schauer RJ, Dugas M, Schachtner T, Kaufmann I, et al. Alpha-gluthathione S-transferase as an early marker of hepatic ischemia/reperfusion injury after liver resection. World J Surg 2005; 29: 528-34.

5. van den Broek MA, Bloemen JG, Dello SA, van de Poll MC, Olde Damink SW, Dejong CH. Randomized controlled trial analyzing the effect of 15 or 30 min intermittent Pringle maneuver on hepatocellular damage during liver surgery. J Hepatol 2011; 55: 337-45.

6. van de Poll MC, Hanssen SJ, Berbee M, Deutz NE, Monbaliu D, Buurman WA, et al. Elevated plasma arginase-1 does not affect plasma arginine in patients undergoing liver resection. Clin Sci (Lond) 2008; 114: 231-41.

7. Liu CL, Fan ST, Cheung ST, Lo CM, Ng IO, Wong J. Anterior approach versus conventional approach right hepatic resection for large hepatocellular carcinoma: a prospective randomized controlled study. Ann Surg 2006; 244: 194-203.

8. Schemmer P, Schoonhoven R, Swenberg JA, Bunzendahl H, Thurman RG. Gentle in situ liver manipulation during organ harvest decreases survival after rat liver transplantation: role of Kupffer cells. Transplantation 1998; 65: 1015-20.

9. Schemmer P, Bunzendahl H, Raleigh JA, Thurman RG. Graft survival is improved by hepatic denervation before organ harvesting. Transplantation 1999; 67: 1301-7.

10. Schemmer P, Enomoto N, Bradford BU, Bunzendahl H, Raleigh JA, Thurman RG. Autonomic nervous system and gut-derived endotoxin: involvement in activation of Kupffer cells after in situ organ manipulation. World J Surg 2001; 25: 399-406.

11. Schemmer P, Connor HD, Arteel GE, Raleigh JA, Bunzendahl H, Mason RP, et al. Reperfusion injury in livers due to gentle in situ organ manipulation during harvest involves hypoxia and free radicals. J Pharmacol Exp Ther 1999; 290: 235-40.

12. Couchoud C, Pozet N, Labeeuw M, Pouteil-Noble C. Screening early renal failure: cut-off values for serum creatinine as an indicator of renal impairment. Kidney Int 1999; 55: 1878-84.

13. Strasberg SM. Nomenclature of hepatic anatomy and resections: a review of the Brisbane 2000 system. J Hepatobiliary Pancreat Surg 2005; 12: 351-5.

14. van den Broek MA, van Dam RM, van Breukelen GJ, Bemelmans MH, Oussoultzoglou E, Pessaux P, et al. Development of a composite endpoint for randomized controlled trials in liver surgery. $\mathrm{Br} J$ Surg 2011; 98: 1138-45.

15. van Dam RM, Hendry PO, Coolsen MM, Bemelmans MH, Lassen K, Revhaug A, et al. Initial experience with a multimodal enhanced recovery programme in patients undergoing liver resection. $\mathrm{Br}$ J Surg 2008; 95: 969-75. 
16. Pelsers MM, Morovat A, Alexander GJ, Hermens WT, Trull AK, Glatz JF. Liver fatty acid-binding protein as a sensitive serum marker of acute hepatocellular damage in liver transplant recipients. Clin Chem 2002; 48: 2055-7.

17. Glatz JF, van der Vusse GJ. Cellular fatty acid-binding proteins: their function and physiological significance. Prog Lipid Res 1996; 35: 243-82.

18. Rensen SS, Slaats Y, Nijhuis J, Jans A, Bieghs V, Driessen A, et al. Increased hepatic myeloperoxidase activity in obese subjects with nonalcoholic steatohepatitis. Am J Pathol 2009; 175: 1473-82.

19. Scatton O, Zalinski S, Jegou D, Compagnon P, Lesurtel M, Belghiti J, et al. Randomized clinical trial of ischaemic preconditioning in major liver resection with intermittent Pringle manoeuvre. $\mathrm{Br} J$ Surg 2011; 98: 1236-43.

20. Petrowsky H, McCormack L, Trujillo M, Selzner M, Jochum W, Clavien PA. A prospective, randomized, controlled trial comparing intermittent portal triad clamping versus ischemic preconditioning with continuous clamping for major liver resection. Ann Surg 2006; 244: 921-8.

21. Sugiyama $Y$, Ishizaki $Y$, Imamura H, Sugo H, Yoshimoto J, Kawasaki S. Effects of intermittent Pringle's manoeuvre on cirrhotic compared with normal liver. Br J Surg 2010; 97: 1062-9.

22. de Jonge WJ, The FO, van der Coelen D, Bennink RJ, Reitsma PH, van Deventer SJ, et al. Mast cell degranulation during abdominal surgery initiates postoperative ileus in mice. Gastroenterology 2004; 127: 535-45.

23. Mantovani A, Sica A, Locati M. New vistas on macrophage differentiation and activation. Eur J Immunol 2007; 37: 14-6.

24. Ramaiah SK, Jaeschke H. Role of neutrophils in the pathogenesis of acute inflammatory liver injury. Toxicol Pathol 2007; 35: 757-766.

25. Nathan C. Points of control in inflammation. Nature 2002; 420: 846-52.

26. Schemmer P, Schoonhoven R, Swenberg JA, Bunzendahl H, Raleigh JA, Lemasters JJ, et al. Gentle organ manipulation during harvest as a key determinant of survival of fatty livers after transplantation in the rat. Transpl Int 1999; 12: 351-9.

27. The FO, Bennink RJ, Ankum WM, Buist MR, Busch OR, Gouma DJ, et al. Intestinal handling-induced mast cell activation and inflammation in human postoperative ileus. Gut 2008; 57: 33-40.

28. The FO, Buist MR, Lei A, Bennink RJ, Hofland J, van den Wijngaard RM, et al. The role of mast cell stabilization in treatment of postoperative ileus: a pilot study. Am J Gastroenterol 2009; 104: 2257-66.

29. The FO, de Jonge WJ, Bennink RJ, van den Wijngaard RM, Boeckxstaens GE. The ICAM-1 antisense oligonucleotide ISIS-3082 prevents the development of postoperative ileus in mice. Br J Pharmacol 2005; 146: 252-8.

30. Lai EC, Fan ST, Lo CM, Chu KM, Liu C. Anterior approach for difficult major right hepatectomy. World J Surg 1996; 20: 314-18.

31. Fan ST. Protection of the liver during partial hepatectomy. Hepatobiliary Pancreat Dis Int 2004; 3: 490-4.

32. Schemmer P, Barro-Bejarano M, Mehrabi A, Gebhard MM, Kraus T, Buchler MW, et al. Laparoscopic organ retrieval for living donor liver transplantation does not prevent graft injury. Transplant Proc 2005; 37: 1625-7.

33. Nsadi B, Gilson N, Pire E, Cheramy J, Pincemail J, Scagnol I, et al. Consequences of pneumoperitoneum on liver ischemia during laparoscopic portal triad clamping in a swine model. J Surg Res 2011; 166: e35-e43.

34. Belghiti J, Guevara OA, Noun R, Saldinger PF, Kianmanesh R. Liver hanging maneuver: a safe approach to right hepatectomy without liver mobilization. J Am Coll Surg 2001; 193: 109-11. 
35. DeLeve LD, Wang X, Kanel GC, Ito Y, Bethea NW, McCuskey MK, et al. Decreased hepatic nitric oxide production contributes to the development of rat sinusoidal obstruction syndrome. Hepatology 2003; 38: 900-8.

36. Mehrabi A, Golling M, Busch C, Hashemi B, Ahmadi R, Volk A, et al. Experimental monitoring of hepatic glucose, lactate, and glutamate metabolism by microdialysis during surgical preparation of the liver hilus. J Surg Res 2002; 105: 128-35.

37. van den Broek MA, van Dam RM, Malago M, Dejong CH, van Breukelen GJ, Olde Damink SW. Feasibility of randomized controlled trials in liver surgery using surgery-related mortality or morbidity as endpoint. Br J Surg 2009; 96: 1005-1014. 



\section{Chapter 6}

\section{Randomized controlled trial analyzing the effect of 15 or 30 min intermittent Pringle maneuver on hepatocellular damage during liver surgery}

Published as:

van den Broek MA, Bloemen JG, Dello SA, van de Poll MC, Olde Damink SW, Dejong $\mathrm{CH}$. Randomized controlled trial analyzing the effect of 15 or $30 \mathrm{~min}$ intermittent Pringle maneuver on hepatocellular damage during liver surgery. 


\section{ABSTRACT}

\section{Background}

Aminotransferases are commonly used to determine the optimal duration of ischemic intervals during an intermittent Pringle maneuver (IPM). However, they might not be responsive enough to detect small differences in hepatocellular damage. Liver fatty acid-binding protein (L-FABP) has been suggested as a more sensitive marker. This randomized trial aimed to compare hepatocellular injury reflected by L-FABP in patients undergoing liver resection with IPM using 15 or 30 min ischemic intervals.

\section{Methods}

Twenty patients undergoing liver surgery were randomly assigned to IPM with 15 (15IPM) or 30 (30IPM) min ischemic intervals. Ten patients not requiring IPM (nolPM) served as controls. Primary endpoint was hepatocellular injury during liver surgery reflected by systemic L-FABP plasma levels. Between group comparisons were performed using area under the curve analysis and repeated measures two-way ANOVA.

\section{Results}

The IPM groups had similar characteristics. Aminotransferases did not differ significantly between 15IPM and 30IPM at any time point. L-FABP levels rose up to $1853 \pm 708 \mathrm{ng} /$ $\mathrm{mL}$ in the $15 \mathrm{IPM}$ and $3662 \pm 1355 \mathrm{ng} / \mathrm{mL}$ in the 30IPM group after finishing liver transection and decreased rapidly thereafter. There were no significant differences between 15IPM and 30IPM in cumulative L-FABP level $(p=0.378)$ or L-FABP level at any time point $(p=0.149)$. Blood loss, remnant liver function and morbidity were comparable.

\section{Conclusion}

IPM with 15 or 30 min ischemic intervals induced similar hepatocellular injury measured by the sensitive marker L-FABP. The present study confirms the results of earlier trials, suggesting that IPM with 30 min ischemic intervals may be used. 


\section{INTRODUCTION}

Hepatic inflow occlusion (Pringle maneuver) is used to minimize blood loss during liver surgery, as excessive intra-operative blood loss and red blood cell transfusions adversely affect short- and long-term outcomes ${ }^{1-3}$. Different clamping techniques can be applied, such as partial or complete hepatic inflow occlusion using either continuous or intermittent pedicle clamping ${ }^{4}$. Generally, intermittent clamping is regarded superior to continuous clamping as it results in a better preserved remnant liver function ${ }^{5}$. The optimal duration of the ischemic intervals during an intermittent Pringle maneuver (IPM) is unknown and depends on the balance between ischemia-induced hepatocellular damage and blood loss. As each period of reperfusion is associated with increased blood $\operatorname{loss}^{5-7}$, prolonged ischemic intervals might be preferable. Indeed, two randomized trials showed that complete IPM using $30 \mathrm{~min}$ ischemic intervals resulted in similar remnant liver function and hepatocellular damage compared to IPM using $15 \mathrm{~min}$ ischemic intervals, while intra-operative blood loss was lower in the $30 \mathrm{~min}$ ischemic interval groups $\mathrm{s}^{6,8}$.

Hepatocellular damage after pedicle clamping is commonly evaluated using alanine and aspartate aminotransferase (ALAT and ASAT) levels on consecutive postoperative days ${ }^{7-9}$. However, it remains uncertain if the assay of aminotransferases is sufficiently sensitive to detect small differences in hepatocellular injury 9 . Due to the relatively large molecular mass ( $96 \mathrm{kDa}$ ) and long half-life of aminotransferases, their plasma levels react slowly to acute tissue damage. In addition, aminotransferase levels on the first postoperative days may not only reflect ischemia-induced hepatocellular damage, but also effects of postoperative care such as drug-induced hepatotoxicity or blood transfusions. Furthermore, aminotransferases, and especially ASAT, have modest organ specificity ${ }^{10}$. More accurate markers for the detection and monitoring of hepatocellular injury in man are available. One of these markers is liver fatty acid-binding protein (L-FABP) ${ }^{11,12}$. L-FABP is a cytosolic protein that is abundantly present in liver tissue. Its biological function involves facilitation of intracellular fatty acid transport and participation in lipid metabolism ${ }^{12}$. After hepatocyte damage, it diffuses quickly into the circulation because of its small mass $(\approx 13-14 \mathrm{kDa}$ ). Circulating L-FABP, released from damaged cells, is cleared by the kidneys with a half-life of $11 \mathrm{~min}$ and as a result, plasma levels rapidly return to normal ${ }^{13}$.

The present randomized controlled trial aimed to assess the effect of complete IPM using either 15 or 30 min ischemic intervals on hepatocellular injury reflected by L-FABP as opposed to the less sensitive damage markers ALAT and ASAT. 


\section{METHODS}

\section{Experimental design}

Consecutive patients scheduled to undergo liver surgery at Maastricht University Medical Centre and willing to participate were enrolled in this prospective randomized controlled trial. Exclusion criteria were (1) presence of liver cirrhosis confirmed by biopsy, (2) concomitant extra-hepatic procedures or bilio-enteric anastomosis, (3) steroid hormone medication, (4) renal dysfunction defined as serum creatinine above $137 \mu \mathrm{mol} / \mathrm{L}$ in men and above $104 \mu \mathrm{mol} / \mathrm{L}$ in women ${ }^{14}$, and (5) laparoscopic liver resection.

Immediately after the surgeon decided complete IPM would be required during liver transection, patients were randomized in a 1:1 ratio to receive either IPM with $15 \mathrm{~min}$ ischemic intervals (15IPM) or $30 \mathrm{~min}$ ischemic intervals (30IPM), both followed by $5 \mathrm{~min}$ reperfusion. Randomization was performed in the operating theatre by a researcher using numbered, sealed, opaque envelopes. An independent researcher generated the randomization sequence. Patients were blinded to the allocated intervention. Patients who did not require IPM (noIPM) served as controls.

The study protocol was approved by the Medical Ethics Committee of Maastricht University Medical Centre and registered at ClinicalTrials.gov NCT01099475. The manuscript complies with the updated CONSORT guidelines ${ }^{15}$. Informed consent was obtained prior to surgery.

\section{Surgical procedure}

Patients were anaesthetized using propofol and isoflurane. They routinely had an epidural catheter, urinary catheter, two peripheral venous catheters and indwelling catheters in a jugular vein and radial artery. Body temperature was maintained using a Bair Hugger system (Arizant Healthcare Inc., Eden Prairie, MN, USA). The surgical procedure was performed using a subcostal bilateral incision and Olivier retractors to improve exposure $^{16}$. After dissection of the teres hepatis ligament, the liver was mobilized. Thereafter, an intra-operative ultrasound was performed to define the position of the tumour in relation to vascular and biliary structures. As IPM was not routinely applied, a patient was randomized for 15IPM or 30IPM only after the surgeon had decided a complete Pringle maneuver would be required. During 15IPM or 30IPM, the complete portal triad was clamped using a rubber sling. The time of inflow occlusion was adapted to the need according to the randomization protocol. Occasionally, the left or right pedicle was ligated after protocolled IPM. Five min reperfusion intervals were applied during which transection was stopped and cut surfaces were gently compressed to ensure hemostasis. A Cavitron Ultrasonic Surgical Aspirator (CUSA system 200 macrodissector, Cavitron Surgical Systems, Stamford, CT, USA) and Argon beam coagulation (Force GSU System, Valleylab, Boulder, CO, USA) were used for liver transection. A stapler device or clamps 
were used for transection of the hepatic veins. Central venous pressure was maintained below $5 \mathrm{mmHg}$ during transection to reduce venous back-bleeding. After surgery, the weight of the resection specimen was recorded. Perioperative care was protocolled, as described earlier ${ }^{17}$.

\section{Blood sampling}

Arterial blood samples were drawn from the radial artery catheter according to a fixed protocol (Figure 6.1). Before and after parenchymal transection, blood was sampled from the portal vein and one of the hepatic veins from the non-tumorous side of the liver by direct puncture. Blood samples were transferred to prechilled EDTA tubes (Vacutainer, Becton Dickinson, Franklin Lakes, NJ, USA). The tubes were centrifuged at $4^{\circ} \mathrm{C}$ at $3500 \times \mathrm{g}$ for $15 \mathrm{~min}$ to separate plasma and stored in aliquots at $-80^{\circ} \mathrm{C}$ till batch analysis.

\section{Outcome measures}

The primary endpoint was hepatocellular damage reflected by systemic L-FABP plasma levels during and after liver surgery. Secondary endpoints were hepatocellular damage reflected by ALAT and ASAT, hepatic function reflected by total bilirubin level and prothrombin time, cumulative ischemia and reperfusion time, duration of operation, amount of intra-operative blood loss, blood loss per gram resected liver weight, need for red blood cell transfusion during surgery or within $48 \mathrm{hr}$ after surgery, and morbidity and 30-day mortality. Resections were divided into major (3 or more segments) or minor (less than 3 segments or non-anatomical resections) ${ }^{18}$. The clinical course of the participants was studied prospectively. Postoperative complications were graded according to the Clavien-Dindo score ${ }^{19}$. Grades 1 and 2 were regarded as minor and grades 3, 4 and 5 as major morbidity. Post-resectional liver failure was defined according to the $50-50$

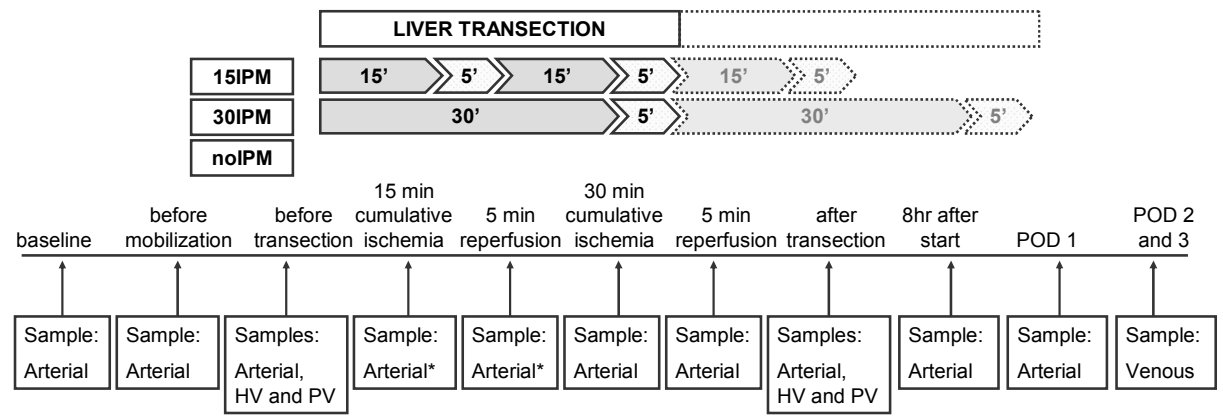

Figure 6.1 Study and sampling protocol.

Legend: 15IPM, 15 min intermittent Pringle maneuver; 30IPM, 30 min intermittent Pringle maneuver; nolPM, no intermittent Pringle maneuver; HV, hepatic vein; PV, portal vein; POD, postoperative day; * only in $15 I P M$ and nolPM group. 
criteria on postoperative day $5^{20}$. Bile leakage was considered in case of (1) leakage of any quantity of bile via the abdominal wound or drain at least $48 \mathrm{hr}$ postoperatively; (2) intra-abdominal collection of bile at the time of re-operation or percutaneous drainage; or (3) cholangiographic evidence of contrast leakage. Intra-abdominal abscess was present if there was (1) leakage of any quantity of purulent fluid via the abdominal drain or (2) intra-abdominal collection of pus at the time of re-operation or percutaneous drainage, both combined with a positive microbiological culture.

\section{Determination of hepatocellular damage}

L-FABP was used as a marker of liver tissue damage. L-FABP plasma levels were determined using a commercially available ELISA (Hycult Biotechnology, Uden, the Netherlands). According to the manufacturer's manual, L-FABP plasma levels in healthy individuals were approximately $12 \mathrm{ng} / \mathrm{mL}$. ALAT and ASAT levels were determined by the clinical chemistry laboratory of Maastricht University Medical Centre. The upper limit of normal was $35 \mathrm{IU} / \mathrm{L}$ for ALAT and $30 \mathrm{IU} / \mathrm{L}$ for ASAT.

\section{Source of L-FABP before and after IPM}

L-FABP is highly expressed in the liver, intestine and kidneys ${ }^{12}$. As a result, systemic L-FABP plasma levels during liver surgery can reflect either hepatic, intestinal or renal damage ${ }^{21}$. The source of systemic L-FABP plasma levels was therefore determined by sampling blood from the portal and hepatic vein simultaneously with an arterial sample and then by calculation of arteriovenous differences $(\triangle A V)$ and net organ fluxes ( $F$; flow $x \triangle A V$ ) across the splanchnic area, portal drained viscera (PDV) and liver. Plasma flow was measured in a previous similar series of patients and amounted to $320 \pm 42 \mathrm{~mL} /$ $\mathrm{min}$ in the portal vein and $110 \pm 23 \mathrm{~mL} / \mathrm{min}$ in the hepatic artery ${ }^{16}$. Splanchnic flow was calculated as portal vein plus hepatic artery plasma flow. Fluxes were calculated as $\mathrm{F}_{\mathrm{PDV}}=$ portal plasma flow * ([portal vein] - [artery]), $\mathrm{F}_{\text {splanchnic }}=$ splanchnic plasma flow * ([hepatic vein] - [artery]) and $F_{\text {liver }}=F_{\text {splanchnic }}-F_{P D V}$ and corrected for body weight. Positive fluxes indicate release, whilst negative fluxes indicate uptake.

\section{Liver histology}

The presence of underlying disease in the non-tumorous liver was assessed by an experienced HPB pathologist in the resection specimen using haematoxylin and eosin staining. The presence of more than 30 per cent hepatic steatosis, grades 1 to 3 fibrosis and nodular regenerative hyperplasia was evaluated in liver tissue distant from the tumour.

\section{Statistical analysis}

Based on previous observations, mean L-FABP plasma level after transection using IPM with $15 \mathrm{~min}$ ischemic and 5 min reperfusion intervals was $775 \pm 210 \mathrm{ng} / \mathrm{mL}^{13}$. The 
present study was powered to detect a 100 per cent relative difference in L-FABP levels between the groups with 15 or 30 min ischemic intervals, favouring the 15 min group. The 100 per cent difference was chosen because it was regarded clinically relevant and precluded large influences of analytical variation. Assuming $a=0.05$ and $\beta=0.2$ with two-sided testing, 10 patients would be required in each study arm.

Continuous data are expressed as mean and standard error if normally distributed or median and range if not normally distributed. Dichotomous data are presented as absolute numbers.

Differences in baseline, operative characteristics and postoperative outcomes between the 15IPM and 30IPM group were tested using a Mann-Whitney $U$ test. Dichotomous data were compared using Pearson's chi-square test. Continuous variables with repeated measurements were summarized as area under the curve (AUC) from baseline to postoperative day $3^{22}$. AUCs of the 15IPM and 30IPM group were compared using the Student's t-test. Subsequently, the two IPM groups were compared with the nolPM group. As a third step, differences in hepatocellular damage markers at specific time points within and between the 15IPM and 30IPM group were analyzed using the repeated measures two-way ANOVA with Bonferroni correction if the $p$-value was below 0.050. L-FABP fluxes before and after liver transection were tested using a one-sample t-test with a theoretical mean of zero. A p-value below 0.050 was considered statistically significant.

\section{RESULTS}

\section{Participant flow}

One-hundred and two patients were assessed between October 2007 and July 2009, of whom 60 were potentially eligible for inclusion. A number of patients who did meet the inclusion criteria were not eligible because of a simultaneous randomized trial on enhanced recovery after surgery. Out of these 60 patients, 30 patients were included in the present study consisting of 10 patients in the 15IPM group, 10 patients in the 30 IPM group and 10 patients in the control group (Figure 6.2). All patients received the allocated intervention. Of the 20 IPM patients, three needed more than two-times 15 min and five more than one-time 30 min intermittent inflow occlusion. In the nolPM group, five patients underwent a liver resection without any form of inflow occlusion and five with pedicle ligation to either the left or right side. No unplanned complete hepatic inflow occlusion was required in this group. No adverse events related to the intervention were encountered. 


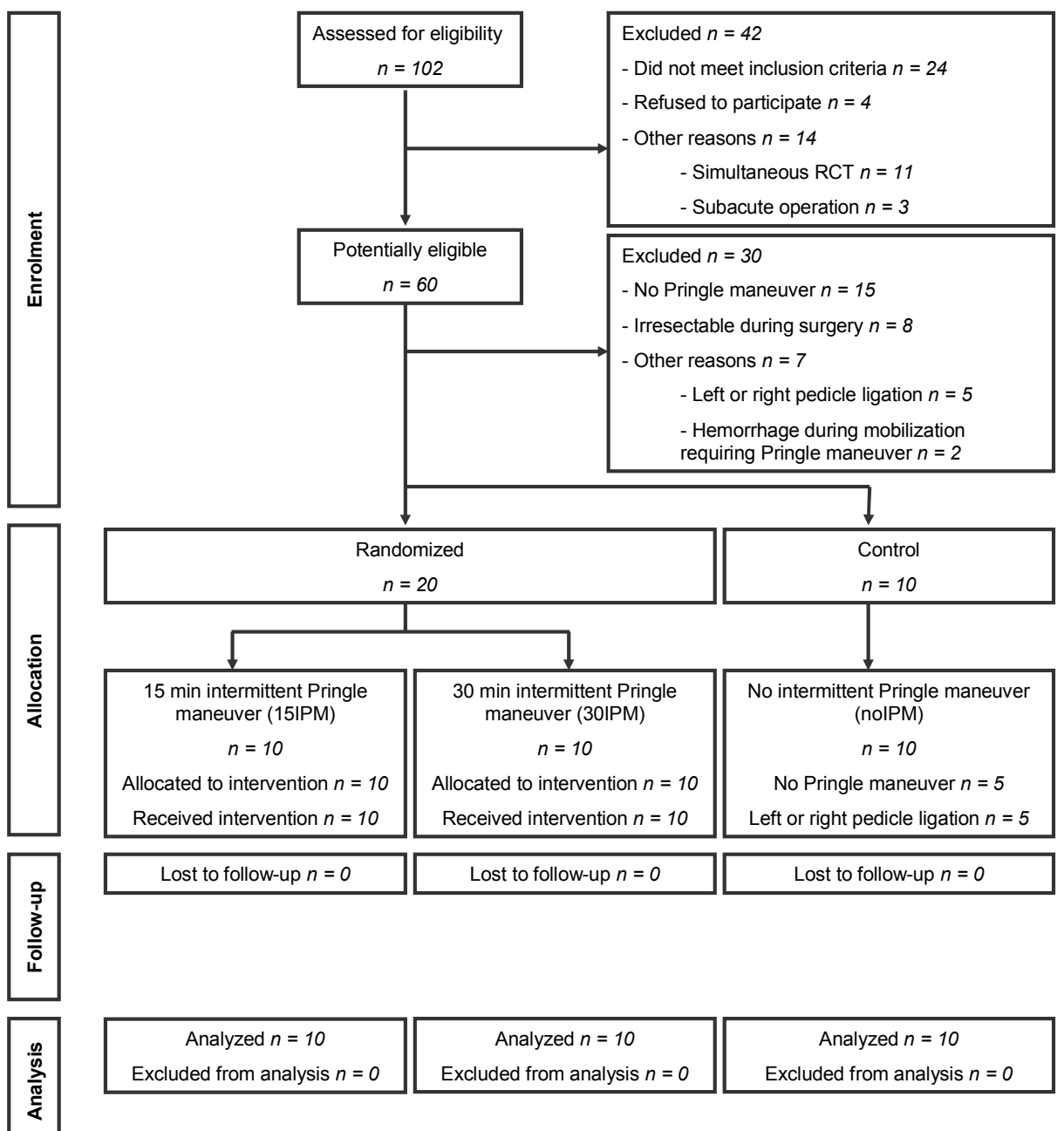

Figure 6.2 CONSORT diagram of patient selection.

Legend: $N$, number; RCT, randomized controlled trial; 15IPM, 15 min intermittent Pringle maneuver; 30IPM, 30 min intermittent Pringle maneuver; nolPM, no intermittent Pringle maneuver.

\section{Patient characteristics}

Table 6.1 shows the patients' demographics and operative characteristics. The indications for liver resection were colorectal liver metastases in 26 patients, primary liver cancer in non-cirrhotic liver in two patients and hepatic adenoma and liver metastases from a carcinoid tumour in one patient each. There were no differences in baseline or operative characteristics between the 15IPM and 30IPM group, except for baseline total bilirubin level, which was significantly higher in the 15IPM group (Table 6.1). The cumulative ischemia time was $34 \mathrm{~min}$ (28-105 $\mathrm{min}$ ) in the 15IPM and $51 \mathrm{~min}(30-65 \mathrm{~min})$ in the 
Table 6.1 Characteristics of patients included in the present randomized controlled trial.

\begin{tabular}{|c|c|c|c|c|}
\hline & \multirow{2}{*}{$\begin{array}{l}\text { Control group } \\
\operatorname{nolPM}(n=10)\end{array}$} & \multicolumn{3}{|c|}{ Intervention groups } \\
\hline & & $15 \mathrm{IPM}(n=10)$ & $301 P M(n=10)$ & p-value* \\
\hline \multicolumn{5}{|l|}{ Baseline characteristics } \\
\hline Age (years) & $60(41-70)$ & $64(48-80)$ & $62(30-77)$ & 0.631 \\
\hline Sex (male) & 7 & 6 & 6 & 1.000 \\
\hline BMI $\left(\mathrm{kg} / \mathrm{m}^{2}\right)$ & $24(22-31)$ & $24(20-31)$ & $26(20-33)$ & 0.684 \\
\hline ASA classification & & & & 0.443 \\
\hline ASA I & 2 & 0 & 2 & \\
\hline ASA II & 8 & 8 & 5 & \\
\hline ASA III & 0 & 2 & 3 & \\
\hline \multicolumn{5}{|l|}{ Preoperative laboratory tests } \\
\hline Bilirubin (total) $(\mu \mathrm{mol} / \mathrm{L})$ & $15(7-37)$ & $14(11-17)$ & $10(8-16)$ & 0.046 \\
\hline Prothrombin time (sec) & $11(10-11)$ & $11(10-14)$ & $10(10-11)$ & 0.348 \\
\hline Creatinine $(\mu \mathrm{mol} / \mathrm{L})$ & $90(54-103)$ & $89(59-128)$ & $83(68-136)$ & 0.617 \\
\hline Indication for resection & & & & 1.000 \\
\hline Benign lesions & 0 & 0 & 1 & \\
\hline Malignant lesions & 10 & 10 & 9 & \\
\hline \multicolumn{5}{|l|}{ Operative characteristics } \\
\hline Type of resection & & & & 0.650 \\
\hline Major hepatectomy & 3 & 5 & 3 & \\
\hline Minor hepatectomy & 7 & 5 & 7 & \\
\hline Number of resected segments & $2(1-5)$ & $2(1-4)$ & $2(1-5)$ & 0.964 \\
\hline Resected liver weight (grams) & $224(51-1065)$ & $317(95-934)$ & $266(41-1140)$ & 0.764 \\
\hline Duration of surgery (min) & $195(150-345)$ & $218(120-315)$ & $235(165-330)$ & 0.323 \\
\hline Mobilization time (min) & $48(20-85)$ & $55(40-130)$ & $55(25-105)$ & 0.762 \\
\hline Transection time (min) & $78(55-160)$ & 75 (40-155) & $110(31-146)$ & 0.315 \\
\hline Cumulative ischemia time (min) & 0 & $34(28-105)$ & $51(30-65)$ & 0.380 \\
\hline Cumulative release time (min) & 0 & $6(4-41)$ & $5(0-14)$ & 0.113 \\
\hline Histology of non-tumorous liver & & & & 1.000 \\
\hline Normal & 7 & 6 & 6 & \\
\hline Steatosis $>30$ per cent & 1 & 1 & 2 & \\
\hline $\mathrm{NRH}$ & 2 & 1 & 1 & \\
\hline Fibrosis grade 1-3 & 0 & 2 & 1 & \\
\hline Intra-operative blood loss (mL) & $500(200-1200)$ & $575(100-2300)$ & $450(250-1000)$ & 0.915 \\
\hline
\end{tabular}

Numbers indicate median (range) or absolute number; N, number; nolPM, no intermittent Pringle maneuver; 15IPM, 15 min intermittent Pringle maneuver; 30IPM, 30 min intermittent Pringle maneuver; BMI, body mass index; ASA, American Society of Anaesthesiologists; $\mathrm{NRH}$, nodular regenerative hyperplasia; * $\mathrm{p}$-value represents comparison between 15IPM and 30IPM group. 
30IPM group ( $p=0.380)$. In the 15IPM group, median blood loss was $575 \mathrm{~mL}(100-2300$ $\mathrm{mL}$ ) compared with $450 \mathrm{~mL}(250-1000 \mathrm{~mL})$ in the 30IPM group $(p=0.915)$. Blood loss per gram resected liver weight was $1.3 \mathrm{~mL} / \mathrm{gram}(0.3-19.0 \mathrm{~mL} / \mathrm{gram})$ in the 15IPM and 1.4 $\mathrm{mL} /$ gram $(0.3-9.8 \mathrm{~mL} / \mathrm{gram})$ in the 30IPM group $(\mathrm{p}=0.887)$. Also transection times were comparable.

\section{Hepatocellular damage}

The degree of hepatocellular damage reflected by L-FABP, ALAT and ASAT in the 15IPM, 30IPM and nolPM group is depicted in Figures 6.3A, 6.3B and 6.3C. Exact concentrations at different time points are depicted in Appendix 10. Already before parenchymal transection, systemic L-FABP levels were significantly increased compared with baseline levels in all groups. L-FABP levels reached their peak just after transection and rapidly returned to baseline levels after surgery.

Systemic ALAT and ASAT levels showed a different pattern. Aminotransferase levels increased significantly during mobilization of the liver and rose further after transection. ALAT and ASAT peaked $8 \mathrm{hr}$ after the start of surgery and remained elevated during the first postoperative days. The mean AUC of L-FABP from baseline up until 3 days after surgery was $9097 \pm 1781$ in the 15IPM and $11688 \pm 2247$ in the 30IPM group $(p=0.378)$. In the nolPM group, the mean AUC was $6374 \pm 760$, which was significantly less than the mean AUC of the IPM groups ( $p=0.019$ ). For ALAT and ASAT, the mean AUCs were 3196 \pm 797 and $3147 \pm 713$ in the 15 IPM and $3609 \pm 812$ and $3800 \pm 799$ in the 30IPM group, respectively ( $p=0.721$ and $p=0.550)$. The nolPM group showed an AUC for ALAT of 2075 \pm 332 and for ASAT of $2129 \pm 276$ ( $p=0.049$ and $p=0.094$ versus the IPM groups).

Furthermore, there was no significant difference between the 15IPM and 30IPM group in systemic L-FABP levels at any time point (overall p-value two-way repeated measures ANOVA $p=0.149$ ). The same holds for ALAT and ASAT levels (overall $p$-value two-way repeated measures ANOVA $p=0.149$ and $p=0.116$, respectively).

\section{Source of L-FABP before and after IPM}

Before liver transection, there was a significant release of L-FABP from the liver in the 15IPM and nolPM group (Figure 6.4A), indicating that mobilization of the liver induced liver cell damage.

After transection, there was a remarkable difference between L-FABP flux in the groups with 15IPM and 30IPM versus the nolPM group, suggesting the induction of ischemic hepatocellular damage by the Pringle maneuver. There was no evidence for intestinal injury secondary to splanchnic vascular congestion during IPM as the PDV did not significantly release L-FABP after transection with IPM using either 15 or 30 min ischemic intervals (Figure 6.4B). 
A

Hepatocellular damage after 15IPM

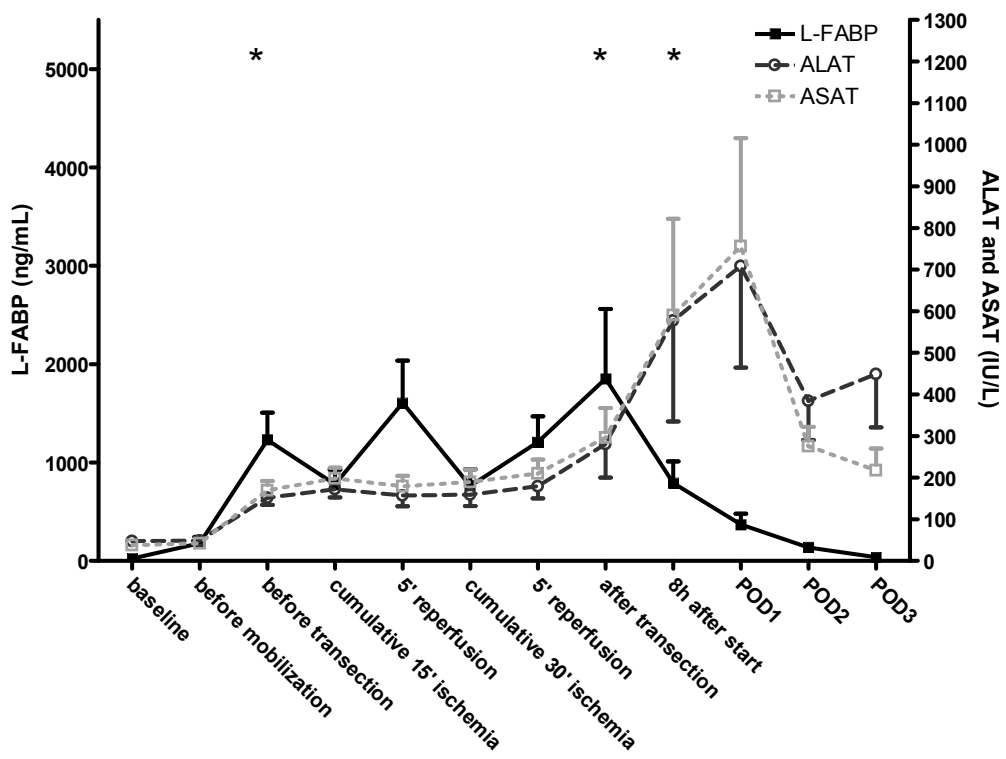

B

Hepatocellular damage after 30IPM

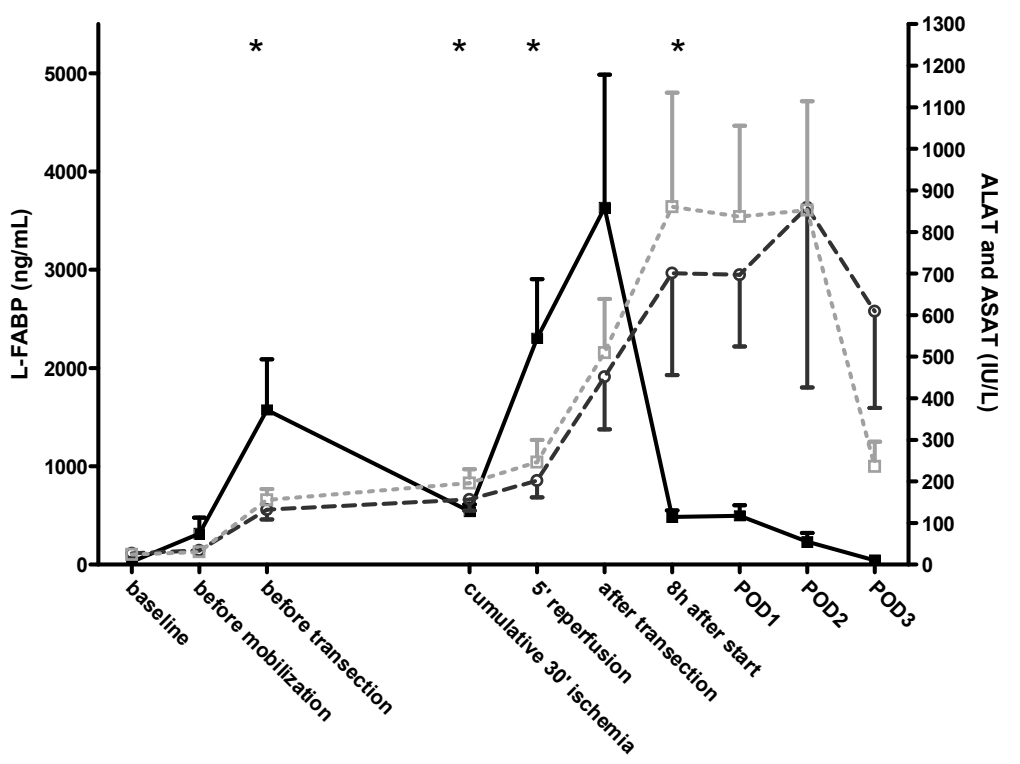


C Hepatocellular damage after noIPM

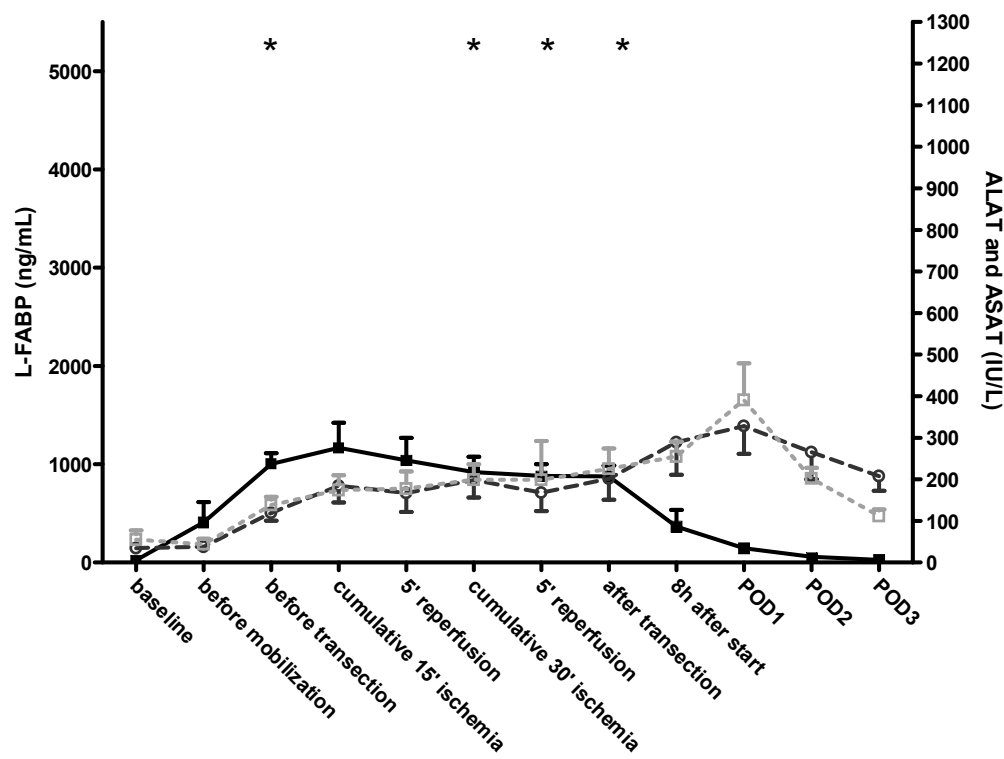

Figure 6.3A-C Course of L-FABP (continuous black line), ALAT (interrupted black line), and ASAT (dotted grey line) in the 15IPM (A), 30IPM (B), and nolPM group (C).

Legend: Data are presented as mean and standard error. Data after cumulative $15^{\prime}$ ischemia and 5 ' reperfusion lack in the 30IPM group; L-FABP, liver fatty acid-binding protein; ALAT, alanine aminotransferase; ASAT, aspartate aminotransferase; 15IPM, 15 min intermittent Pringle maneuver; 30IPM, 30 min intermittent Pringle maneuver; noIPM, no intermittent Pringle maneuver; ${ }^{*} \mathrm{p}<0.050$ for L-FABP versus baseline calculated with repeated measures two-way ANOVA with post-hoc paired sample t-test.

\section{Postoperative course}

The postoperative course is depicted in Table 6.2. Seventeen patients experienced postoperative complications ( 57 per cent), of whom 8 had only minor and nine a combination of minor and major complications. Two patients died within 30 days after liver surgery, accounting for a 30-day mortality rate of 7 per cent. The reasons of death were sepsis with multi-organ failure and post-resectional liver failure in 1 patient each. The 30-day mortality in the total cohort of patients $(n=102)$ who had undergone liver resection during the inclusion period of the present study was 4 per cent. The complications in the two IPM groups were comparable. Four patients in the 15IPM group and two in the 30IPM group required a red blood cell transfusion $(p=0.628)$. 
A

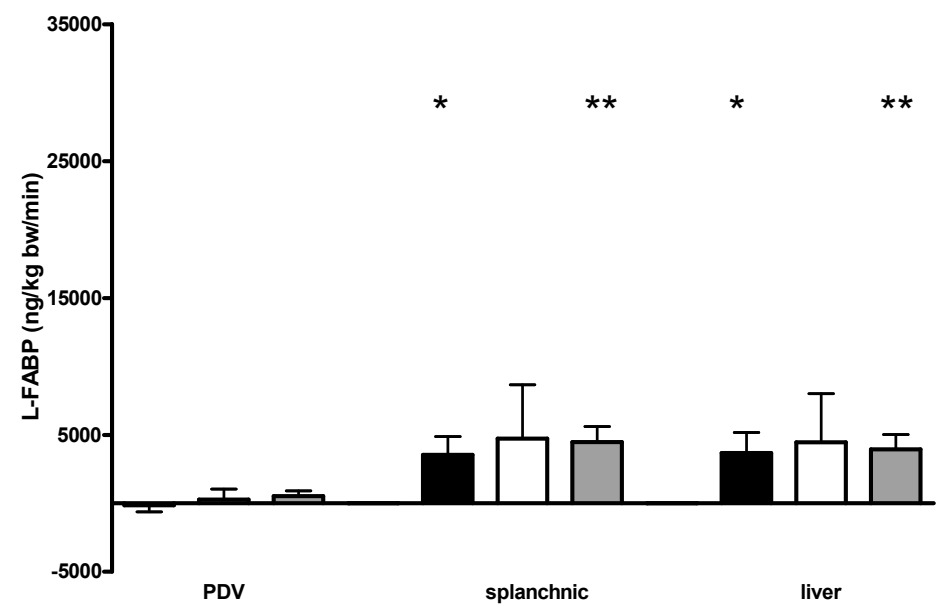

B

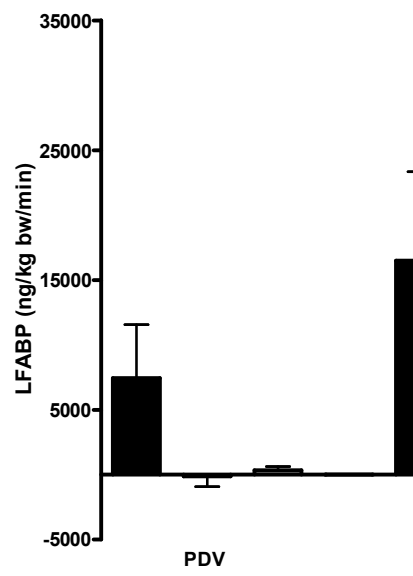

Flux L-FABP after IPM

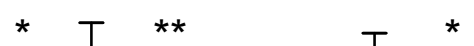

-15IPM

口nolPM
口30IPM

$*$
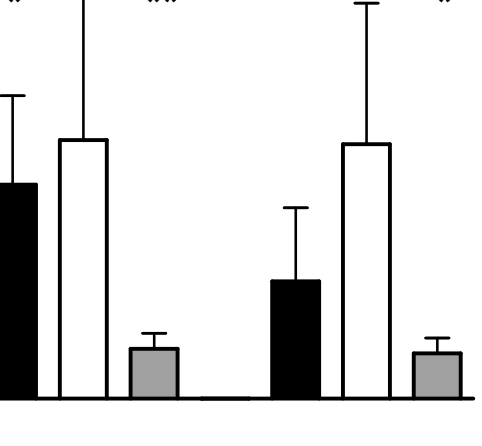

splanchnic

liver

Figure 6.4A and 6.4B Fluxes of L-FABP before and after liver transection with and without intermittent Pringle maneuver.

Legend: Data are presented as mean and standard error; PDV, portal drained viscera; L-FABP, liver fatty acidbinding protein; IPM, intermittent Pringle maneuver; ${ }^{*} p<0.050$ and ${ }^{* *} p<0.010$.

\section{DISCUSSION}

The present randomized controlled trial was designed to compare the effect of intermittent hepatic inflow occlusion using either 15 or $30 \mathrm{~min}$ ischemic intervals on hepatocellular damage during liver surgery in man. In addition to the commonly used aminotransferases, the more sensitive marker L-FABP was studied as a read out for liver injury. 
Table 6.2 Postoperative course of patients undergoing liver surgery with or without intermittent Pringle maneuver.

\begin{tabular}{|c|c|c|c|c|}
\hline & \multirow{2}{*}{$\begin{array}{l}\text { Control group } \\
\text { nolPM }(n=10)\end{array}$} & \multicolumn{3}{|c|}{ Intervention groups } \\
\hline & & $15 \mathrm{IPM}(n=10)$ & $30 \mathrm{IPM}(n=10)$ & $p$-value* \\
\hline \multicolumn{5}{|l|}{ Postoperative liver function } \\
\hline Peak bilirubin (total) $(\mu \mathrm{mol} / \mathrm{L})$ & $23(16-101)$ & $31(22-194)$ & $37(14-84)$ & 0.670 \\
\hline Peak prothrombin time (sec) & $13(11-21)$ & $14(11-62)$ & $15(11-21)$ & 0.324 \\
\hline \multicolumn{5}{|l|}{ Minor complications" } \\
\hline Pneumonia & 1 & 3 & 1 & 0.582 \\
\hline Pleural effusion & 1 & 2 & 1 & 1.000 \\
\hline Wound infection & 1 & 1 & 0 & 1.000 \\
\hline lleus & 1 & 1 & 1 & 1.000 \\
\hline Red blood cell transfusion & 1 & 4 & 2 & 0.628 \\
\hline Units & 1 (n.a.) & $4(2-7)$ & $2(1-2)$ & 0.267 \\
\hline \multicolumn{5}{|l|}{ Major complications ${ }^{\#}$} \\
\hline Intra-abdominal abscess & 0 & 3 & 2 & 1.000 \\
\hline Intra-abdominal hemorrhage & 0 & 0 & 1 & 1.000 \\
\hline Bile leakage & 0 & 4 & 1 & 0.303 \\
\hline Ascites & 0 & 1 & 0 & 1.000 \\
\hline Sepsis & 0 & 1 & 2 & 1.000 \\
\hline Post-resectional liver failure & 1 & 2 & 1 & 1.000 \\
\hline 30-day mortality & 0 & 1 & 1 & 1.000 \\
\hline \multicolumn{5}{|l|}{ Postoperative stay } \\
\hline Length of hospital stay (days) & $8(5-10)$ & $11(5-53)$ & $8(5-119)$ & 0.955 \\
\hline
\end{tabular}

Numbers indicate median (range) or absolute number; N, number; nolPM, no intermittent Pringle maneuver; 15IPM, 15 min intermittent Pringle maneuver; 30IPM, 30 min intermittent Pringle maneuver; n.a., not applicable; ${ }^{*}$ graded according to the Clavien-Dindo classification ${ }^{19} ;{ }^{*}$ p-value represents comparison between 15IPM and 30IPM group.

We provide novel data that show no difference in hepatocellular damage reflected by L-FABP between patients undergoing liver resection with complete IPM using either 15 or $30 \mathrm{~min}$ ischemic intervals. These results corroborate results of earlier trials which used less sensitive markers for liver tissue damage. Intra-operative blood loss, red cell blood transfusions and remnant liver function were comparable. Moreover, complete IPM did not induce intestinal injury secondary to portovenous stasis.

Hepatic inflow occlusion is frequently applied during liver transection to prevent excessive blood loss ${ }^{4,23}$. A drawback of the Pringle maneuver is the induction of ischemic hepatocellular injury resulting in postoperative morbidity ${ }^{24,25}$. In order to limit this injury, techniques such as ischemic preconditioning and intermittent pedicle clamping are effective ${ }^{5,7}$. However, several trials suggested that IPM using $15 \mathrm{~min}$ ischemic intervals was associated with significantly higher blood loss during transection compared with 
continuous clamping, resulting from extensive bleeding during the 5 min reperfusion periods $\mathrm{s}^{5,7}$. Theoretically, ischemic intervals of $30 \mathrm{~min}$ are preferable as this time period allows the surgeon to control vessels at the cut surface of the liver meticulously whereas the liver can tolerate cumulative ischemia up to $120 \mathrm{~min}^{26}$. Moreover, short ischemic intervals lead to repetitive reperfusion episodes, potentially resulting in more extensive hepatocellular damage. Two recent randomized trials did show that remnant liver function and hepatocellular damage after IPM using 15 or 30 min ischemic intervals were comparable, whereas blood loss was lower after $30 \mathrm{IPM}^{6,8}$. In these studies, however, bilirubin or aminotransferase levels on different postoperative days were used as a read out for liver function and damage while it is questionable whether these levels are sufficiently sensitive to pick up slight differences between the two ischemic intervals ${ }^{10}$. Our trial confirms that hepatocellular damage, measured by the highly sensitive damage marker L-FABP, was similar between complete IPM with 15 or 30 min ischemic intervals. Also remnant liver function was comparable. However, no IPM resulted in significantly less hepatocellular damage in the control group of patients with similar characteristics. As a recent meta-analysis showed no definite advantage of any form of portal triad clamping in terms of postoperative outcomes, IPM should be preserved for individual patients undergoing complex resections with expected high blood loss ${ }^{27}$. If a Pringle maneuver is necessary, $30 \mathrm{~min}$ ischemic intervals should be used as this led to decreased blood loss and a higher transection speed in previous studies with larger patient numbers $^{6,8}$.

Another concern of the Pringle maneuver is the induction of intestinal damage secondary to portovenous stasis ${ }^{28}$. Theoretically, intestinal injury after hepatic inflow occlusion might result in intestinal barrier dysfunction and bacterial translocation. The clinical relevance of clamping-induced intestinal injury in man is under debate ${ }^{28,29}$. Kim and colleagues described an increase in portovenous interleukin- 6 levels compared with arterial levels at the end of continuous inflow occlusion, suggesting interleukin- 6 production in the mesenteric area ${ }^{28}$. A small study by Huguet's group showed that, even though bacterial translocation to mesenteric lymph nodes occurred during liver resection under continuous inflow occlusion, no correlation between positive lymph nodes and postoperative infectious complications was present ${ }^{29}$. Our data provide clear evidence that complete IPM using either 15 or 30 min inflow occlusion induced no significant intestinal damage, evidenced by minimal L-FABP fluxes over the portal drained viscera. L-FABP is abundantly present in intestinal villi and intestinal damage typically results in a rapid release of L-FABP ${ }^{21}$. Therefore, L-FABP plasma concentration is regarded a reliable marker for the detection of intestinal injury. The exact relation between L-FABP plasma level and gut barrier function has, to the best of our knowledge, not yet been elucidated. As a result, the effect of IPM with either 15 or 30 min inflow occlusion on intestinal barrier function remains to be established. By measuring fluxes, 
an exclusive hepatic origin of systemic L-FABP plasma levels after liver transection could be confirmed.

Recently, doubts have arisen concerning the contribution of ischemia- reperfusion to total hepatocellular damage caused by liver surgery. Several experimental studies confirm our observation that mobilization of the liver results in profound liver tissue damage, even before inflow occlusion is applied ${ }^{13,30}$. It is hypothesized that liver manipulation results in a disturbed hepatic microcirculation leading to hypoxia and Kupffer cell activation which causes additional hepatic injury ${ }^{31}$. Therefore, interventions aiming at minimization of hepatocellular damage should be started early during liver surgery.

Our study has some limitations. First, the relation between hepatocellular damage reflected by L-FABP or aminotransferases and postoperative outcome is indistinct. The current trial was powered to detect a 100 per cent difference in L-FABP level between the 15IPM and 30IPM group. In order to study differences in postoperative morbidity, a considerably larger number of patients should have been included ${ }^{27,32}$. Furthermore, our results may not apply to patients with underlying liver disease. Insights in the effect of IPM on hepatocellular damage in these patients were reported recently ${ }^{9}$. After liver surgery with inflow occlusion, patients with liver cirrhosis had a lower difference between preoperative and maximum postoperative ASAT and ALAT levels compared with patients with normal liver, while their postoperative remnant liver function was worse. This further underlines the idea that aminotransferases, measured during hospital stay, may not accurately reflect the course of events secondary to hepatic inflow occlusion. Finally, a relation between prolonged pedicle clamping and accelerated outgrowth of residual liver disease was demonstrated in a murine model ${ }^{33}$. Clinical data on this topic in man are contradictory ${ }^{34,35}$. Long-term results of a randomized controlled study that compared liver surgery with or without IPM showed no difference in disease-free or overall survival between the two patient groups ${ }^{36}$.

To conclude, complete IPM using 30 min ischemic intervals resulted in similar hepatocellular damage, reflected by the highly sensitive damage marker L-FABP, compared with complete IPM using 15 min ischemic intervals, without induction of intestinal damage or loss of remnant liver function. If inflow occlusion during liver transection is necessary, intermittent pedicle clamping using $30 \mathrm{~min}$ ischemic intervals may be used. 


\section{REFERENCES}

1. Imamura H, Seyama Y, Kokudo N, Maema A, Sugawara Y, Sano K, et al. One thousand fifty-six hepatectomies without mortality in 8 years. Arch Surg 2003; 138: 1198-206.

2. Kooby DA, Stockman J, Ben-Porat L, Gonen M, Jarnagin WR, Dematteo RP, et al. Influence of transfusions on perioperative and long-term outcome in patients following hepatic resection for colorectal metastases. Ann Surg 2003; 237: 860-9.

3. Gomez D, Morris-Stiff G, Wyatt J, Toogood GJ, Lodge JP, Prasad KR. Surgical technique and systemic inflammation influences long-term disease-free survival following hepatic resection for colorectal metastasis. J Surg Oncol 2008; 98: 371-6.

4. van der Bilt JD, Livestro DP, Borren A, van Hillegersberg R, Borel Rinkes IH. European survey on the application of vascular clamping in liver surgery. Dig Surg 2007; 24: 423-35.

5. Belghiti J, Noun R, Malafosse R, Jagot P, Sauvanet A, Pierangeli F, et al. Continuous versus intermittent portal triad clamping for liver resection: a controlled study. Ann Surg 1999; 229: 369-75.

6. Esaki M, Sano T, Shimada K, Sakamoto Y, Takahashi Y, Wakai K, et al. Randomized clinical trial of hepatectomy using intermittent pedicle occlusion with ischaemic intervals of 15 versus 30 minutes. Br J Surg 2006; 93: 944-51.

7. Petrowsky H, McCormack L, Trujillo M, Selzner M, Jochum W, Clavien PA. A prospective, randomized, controlled trial comparing intermittent portal triad clamping versus ischemic preconditioning with continuous clamping for major liver resection. Ann Surg 2006; 244: 921-8.

8. Kim YI, Fujita S, Hwang YJ, Chun JM, Song KE, Chun BY. Successful intermittent application of the Pringle maneuver for 30 minutes during human hepatectomy: a clinical randomized study with use of a protease inhibitor. Hepatogastroenterology 2007; 54: 2055-60.

9. Sugiyama $Y$, Ishizaki $Y$, Imamura H, Sugo H, Yoshimoto J, Kawasaki S. Effects of intermittent Pringle's manoeuvre on cirrhotic compared with normal liver. Br J Surg 2010; 97: 1062-9.

10. Rej R. Aminotransferases in disease. Clin Lab Med 1989; 9: 667-87.

11. Pelsers MM, Morovat A, Alexander GJ, Hermens WT, Trull AK, Glatz JF. Liver fatty acid-binding protein as a sensitive serum marker of acute hepatocellular damage in liver transplant recipients. Clin Chem 2002; 48: 2055-7.

12. Glatz JF, van der Vusse GJ. Cellular fatty acid-binding proteins: their function and physiological significance. Prog Lipid Res 1996; 35: 243-82.

13. van de Poll MC, Derikx JP, Buurman WA, Peters WH, Roelofs HM, Wigmore SJ, et al. Liver manipulation causes hepatocyte injury and precedes systemic inflammation in patients undergoing liver resection. World J Surg 2007; 31: 2033-8.

14. Couchoud C, Pozet N, Labeeuw M, Pouteil-Noble C. Screening early renal failure: cut-off values for serum creatinine as an indicator of renal impairment. Kidney Int 1999; 55: 1878-84.

15. Schulz KF, Altman DG, Moher D. CONSORT 2010 statement: updated guidelines for reporting parallel group randomised trials. Bmj 2010; 340: 698-702.

16. van de Poll MC, Siroen MP, van Leeuwen PA, Soeters PB, Melis GC, Boelens PG, et al. Interorgan amino acid exchange in humans: consequences for arginine and citrulline metabolism. Am J Clin Nutr 2007; 85: 167-72.

17. van Dam RM, Hendry PO, Coolsen MM, Bemelmans MH, Lassen K, Revhaug A, et al. Initial experience with a multimodal enhanced recovery programme in patients undergoing liver resection. $\mathrm{Br}$ J Surg 2008; 95: 969-75.

18. Strasberg SM. Nomenclature of hepatic anatomy and resections: a review of the Brisbane 2000 system. J Hepatobiliary Pancreat Surg 2005; 12: 351-5. 
19. Dindo D, Demartines N, Clavien PA. Classification of surgical complications: a new proposal with evaluation in a cohort of 6336 patients and results of a survey. Ann Surg 2004; 240: 205-13.

20. Balzan S, Belghiti J, Farges O, Ogata S, Sauvanet A, Delefosse D, et al. The "50-50 criteria" on postoperative day 5: an accurate predictor of liver failure and death after hepatectomy. Ann Surg 2005; 242: 824-8.

21. Pelsers MM, Namiot z, Kisielewski W, Namiot A, Januszkiewicz M, Hermens WT, et al. Intestinaltype and liver-type fatty acid-binding protein in the intestine. Tissue distribution and clinical utility. Clin Biochem 2003; 36: 529-35.

22. Matthews JN, Altman DG, Campbell MJ, Royston P. Analysis of serial measurements in medical research. Bmj 1990; 300: 230-5.

23. Man K, Lo CM, Liu CL, Zhang ZW, Lee TK, Ng IO, et al. Effects of the intermittent Pringle manoeuvre on hepatic gene expression and ultrastructure in a randomized clinical study. Br J Surg 2003; 90: 183-9.

24. Benzoni E, Lorenzin D, Baccarani U, Adani GL, Favero A, Cojutti A, et al. Resective surgery for liver tumor: a multivariate analysis of causes and risk factors linked to postoperative complications. Hepatobiliary Pancreat Dis Int 2006; 5: 526-33.

25. van den Broek MA, Olde Damink SW, Dejong CH, Lang H, Malago M, Jalan R, et al. Liver failure after partial hepatic resection: definition, pathophysiology, risk factors and treatment. Liver Int 2008; 28: 767-80.

26. Man K, Fan ST, Ng IO, Lo CM, Liu CL, Yu WC, et al. Tolerance of the liver to intermittent pringle maneuver in hepatectomy for liver tumors. Arch Surg 1999; 134: 533-9.

27. Rahbari NN, Wente MN, Schemmer P, Diener MK, Hoffmann K, Motschall E, et al. Systematic review and meta-analysis of the effect of portal triad clamping on outcome after hepatic resection. $\mathrm{Br} J$ Surg 2008; 95: 424-32.

28. Kim YI, Song KE, Ryeon HK, Hwang YJ, Yun YK, Lee JW, et al. Enhanced inflammatory cytokine production at ischemia/reperfusion in human liver resection. Hepatogastroenterology 2002; 49: 1077-82.

29. Ferri M, Gabriel S, Gavelli A, Franconeri P, Huguet C. Bacterial translocation during portal clamping for liver resection. A clinical study. Arch Surg 1997; 132: 162-5.

30. Chouker A, Martignoni A, Schauer RJ, Dugas M, Schachtner T, Kaufmann I, et al. Alpha-gluthathione S-transferase as an early marker of hepatic ischemia/reperfusion injury after liver resection. World J Surg 2005; 29: 528-34.

31. Schemmer P, Bunzendahl H, Klar E, Thurman RG. Reperfusion injury is dramatically increased by gentle liver manipulation during harvest. Transpl Int 2000; 13 Suppl 1: S525-7.

32. van den Broek MA, van Dam RM, Malago M, Dejong CH, van Breukelen GJ, Olde Damink SW. Feasibility of randomized controlled trials in liver surgery using surgery-related mortality or morbidity as endpoint. Br J Surg 2009; 96: 1005-1014.

33. van der Bilt JD, Kranenburg O, Nijkamp MW, Smakman N, Veenendaal LM, Te Velde EA, et al. Ischemia/reperfusion accelerates the outgrowth of hepatic micrometastases in a highly standardized murine model. Hepatology 2005; 42: 165-75.

34. Nijkamp MW, van der Bilt JD, Snoeren N, Hoogwater FJ, van Houdt WJ, Molenaar IQ, et al. Prolonged portal triad clamping during liver surgery for colorectal liver metastases is associated with decreased time to hepatic tumour recurrence. Eur J Surg Oncol 2010; 36: 182-8.

35. Wong KH, Hamady ZZ, Malik HZ, Prasad R, Lodge JP, Toogood GJ. Intermittent Pringle manoeuvre is not associated with adverse long-term prognosis after resection for colorectal liver metastases. Br J Surg 2008; 95: 985-9. 
36. Ferrero A, Russolillo N, Vigano L, Lo Tesoriere R, Muratore A, Capussotti L. Does Pringle Manoeuvre Affect Survival in Patients with Colorectal Liver Metastases? World J Surg 2010; 34: 2418-2425. 



\section{Part III}

Assessing outcomes of liver surgery:

hepatic damage as a result of

oxaliplatin-based chemotherapy 



\section{Chapter 7}

\section{Nodular regenerative hyperplasia secondary to neoadjuvant chemotherapy for colorectal liver metastases}

Published as:

van den Broek MA, Olde Damink SW, Driessen A, Dejong $\mathrm{CH}$, Bemelmans $\mathrm{MH}$. Nodular regenerative hyperplasia secondary to neoadjuvant chemotherapy for colorectal liver metastases. Case Reports Med 2009; 2009: 457975. 


\section{ABSTRACT}

Liver resection is the only curative treatment for patients with colorectal liver metastases (CLMs). Chemotherapy can improve resectability rates, but has a potential harmful effect on the non-tumour-bearing liver. Patients with chemotherapy-induced hepatic injury undergoing major liver surgery have higher risks of post-resectional morbidity. We present two cases of patients without pre-existent liver disease treated with oxaliplatinbased chemotherapy followed by surgical resection of their CLMs. Their intra-operative liver specimen showed morphologic abnormalities characteristic of nodular regenerative hyperplasia (NRH). NRH led to portal hypertension in both patients that resulted in deleterious post-resectional complications and death of one patient. Interestingly, the other patient underwent two repeat non-anatomic liver resections because of recurrent CLMs. The intra-operative liver specimen still showed signs of $\mathrm{NRH}$ and sinusoidal congestion, but the post-resectional courses were uneventful. Nevertheless, caution is recommended in patients with suspected NRH. Careful volumetric analysis should guide the operative strategy. When future remnant liver volume or function are regarded insufficient, portal vein embolization or restrictive surgery should be considered. 


\section{INTRODUCTION}

Colorectal liver metastases (CLMs) develop in 50 to 60 per cent of patients with colorectal carcinoma ${ }^{1}$. Resection remains the only curative treatment, but just a fifth of patients are initially eligible for resection. In these patients, perioperative chemotherapy has proven to elongate progression-free survival ${ }^{2}$. In addition, administration of chemotherapy can improve resectability in 15 to 30 per cent of patients with initially unresectable disease ${ }^{1,3}$. However, recent reports show a potential harmful effect of oxaliplatin and irinotecan-based neoadjuvant chemotherapy on the non-tumour-bearing liver ${ }^{4-6}$. Patients with histologically proven chemotherapy-related hepatic injury undergoing major liver surgery have higher risks of post-resectional morbidity and mortality ${ }^{6,7}$.

Here, we present two cases of patients without pre-existent liver disease preoperatively treated with oxaliplatin and capecitabine because of CLMs, who developed nodular regenerative hyperplasia (NRH) of the liver causing deleterious complications after major hemihepatectomy. Interestingly, one of these patients underwent limited repeat resections after 12 and 18 months because of recurrent CLMs, after which the clinical courses were uneventful.

\section{PATIENT CASES}

\section{Case 1}

A 68-year-old man was referred to our hospital because of synchronous CLMs. His medical history revealed a colorectal carcinoma (pT3NOM1) treated by right hemicolectomy. Neoadjuvant chemotherapy consisting of 6 cycles of oxaliplatin $\left(130 \mathrm{mg} / \mathrm{m}^{2}\right)$ and capecitabine ( $1000 \mathrm{mg} / \mathrm{m}^{2}$ two-times daily) was initiated after resection of the colorectal primary. The patient had no history of liver disease and computed tomography (CT) scan did not reveal any signs of cirrhosis, portal hypertension, parenchymal abnormalities or other liver pathology. Liver function tests prior to and after administration of chemotherapy are depicted in Table 7.1. CT images showed a partial response of the CLMs with metastases remaining in segments IVb, VI and VIII for which an extended right hemihepatectomy was the only realistic curative treatment. As a remnant liver volume of only 18 per cent was calculated by CT volumetry, preoperative right portal vein embolization was performed. Five weeks later, remnant liver volume increased up to 28 per cent which was regarded just sufficient for safe hemihepatectomy. An extended right hemihepatectomy with Roux-Y reconstruction was performed as detailed previously ${ }^{8}$. A bluish appearance of the liver was noticed intra-operatively without signs of portal hypertension. Histological examination of the liver specimen showed sinusoidal congestion and nodularity characteristic of $\mathrm{NRH}$. The postoperative course was uncomplicated 
Table 7.1 Laboratory values of the patients described in case 1 and 2 before and after administration of oxaliplatin-based neoadjuvant chemotherapy.

\begin{tabular}{|c|c|c|c|c|}
\hline & \multicolumn{2}{|c|}{ Case 1} & \multicolumn{2}{|c|}{ Case 2} \\
\hline & Before & After & Before & After \\
\hline Alkaline phosphatase (IU/L) & 205 & 237 & 328 & 205 \\
\hline Y-glutamyltransferase (IU/L) & 50 & 42 & 233 & 90 \\
\hline ASAT (IU/L) & n.a. & 59 & 61 & 79 \\
\hline ALAT (IU/L) & n.a. & 23 & 91 & 57 \\
\hline Bilirubin (total) ( $\mu \mathrm{mol} / \mathrm{L})$ & n.a. & 39 & 12 & 21 \\
\hline INR & n.a. & n.a. & n.a. & n.a. \\
\hline
\end{tabular}

ASAT, aspartate aminotransferase; ALAT, alanine aminotransferase; INR, international standardized ratio; n.a., not available.

till day four. From then on, bilirubin level and prothrombin time increased reflecting post-resectional liver failure. Acute upper gastro-intestinal hemorrhage occurred on day eight for which early management consisted of emergent endoscopy that showed bleeding from grade IV gastro-oesophageal varices. A CT scan performed on the same day showed impressive portacaval collateral shunting in the splanchnic area (Figure 7.1). Unfortunately, hemorrhage recurred several hours later and a second endoscopy failed to control it. Therefore, a Sengstaken-Blakemore tube was inserted and a laparotomy was performed during which the stomach was packed. During relaparotomy, no evidence of liver surgery-related complications was found. Because of ongoing deterioration, a distal splenorenal shunt was created one day later to relieve portal pressure.

Despite these efforts, the patient died several hours after relaparotomy because of multi-organ failure secondary to hemorrhagic shock. Post-mortem evaluation revealed $\mathrm{NRH}$ of the liver combined with hepatic congestion and infarction as well as extensive collateral vessels in the splanchnic region. Again, there was no evidence of surgeryrelated technical failure such as portal or hepatic vein obstruction.

\section{Case 2}

A 51-year-old man consulted our hospital because of synchronous CLMs after sigmoid resection for colorectal carcinoma (pT3N0M1) elsewhere. The patient received six cycles of oxaliplatin $\left(130 \mathrm{mg} / \mathrm{m}^{2}\right)$ and capecitabine $\left(1000 \mathrm{mg} / \mathrm{m}^{2}\right.$ two-times daily) subsequent to resection of his primary tumour. There was no history of liver disease. The evolution of the liver function tests of the patient described in this case is shown in Table 7.1. CT imaging showed tumour regression after chemotherapy with metastases remaining in segments III, IVa and the right hemi-liver. A right hemihepatectomy with metastasectomy of tumour from segments III and IVa was performed without biliary reconstruction. Remnant liver volume was estimated intra-operatively to be 35 per cent with a bluish appearance of the liver remnant. Histological examination of the liver specimen showed 


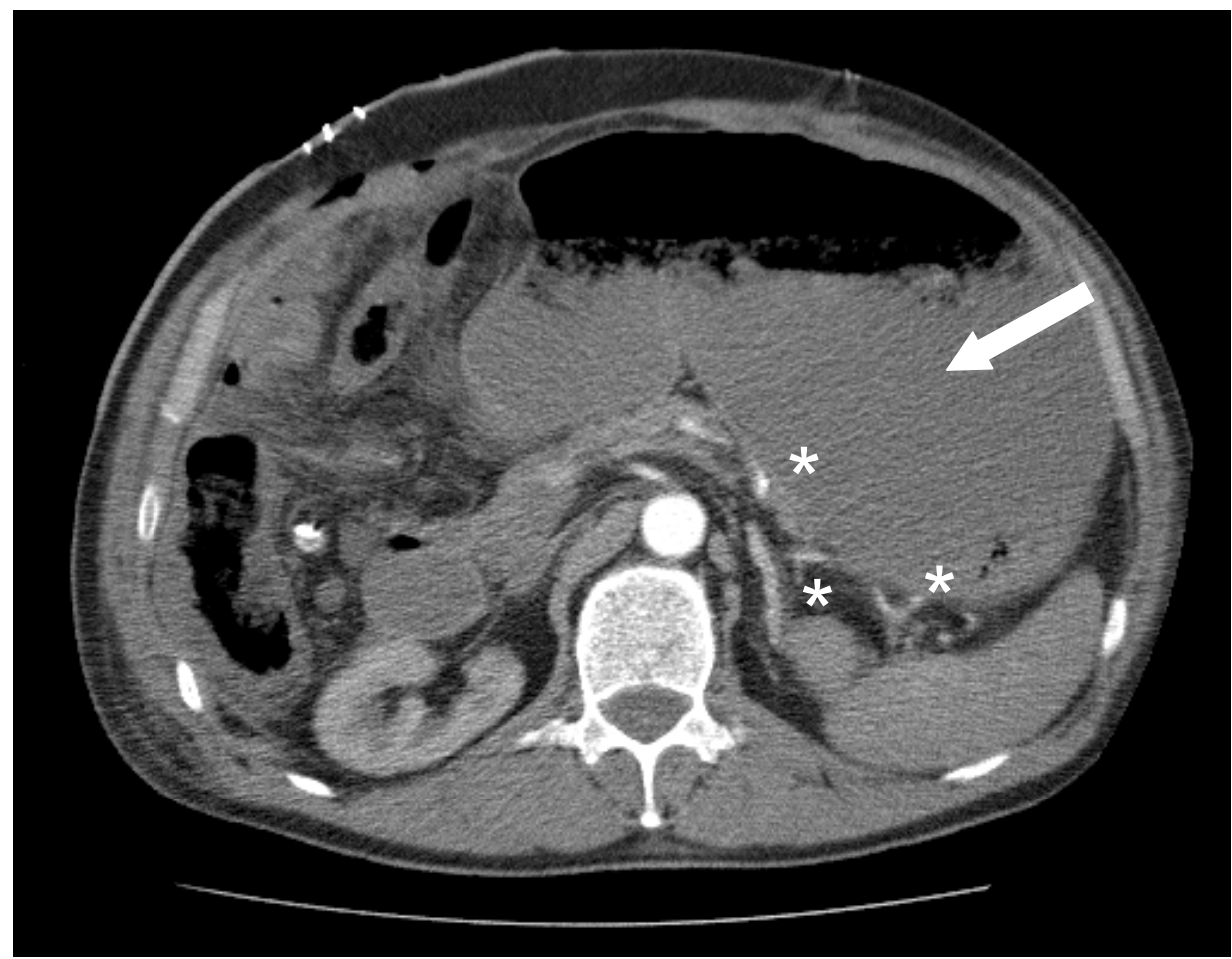

Figure 7.1 Contrast-enhanced CT scan performed 8 days postoperatively (case 1). The stomach (white arrow) is filled with blood after upper gastro-intestinal bleeding from gastro-oesophageal varices secondary to portal hypertension. Multiple, large collateral veins run along the stomach and spleen (white asterisk).

a nodular appearance with hepatic congestion characteristic of NRH (Figure 7.2 and 7.3). The postoperative course was complicated by bile leakage treated by CT guided percutaneous drainage and ERCP guided common bile duct stenting. Bilirubin level and prothrombin time decreased during admission and the patient was discharged 14 days postoperatively. Two days after discharge, the patient was readmitted in his hometown hospital because of portal hypertension leading to oesophageal variceal bleeding with hepatic encephalopathy treated by endoscopic band ligation and conservative measures, respectively. Laboratory values normalized gradually consistent with an improvement of the patient's clinical and mental status. Full recovery was accomplished after two months.

One year afterwards, the patient presented himself with multiple, recurrent CLMs in segments II and III for which he received 3 cycles of irinotecan $\left(300 \mathrm{mg} / \mathrm{m}^{2}\right)$ prior to liver surgery. Preoperative diagnostic liver biopsy showed minimal nodularity and steatosis without indications for $\mathrm{NRH}$ or steatohepatitis. The patient underwent treatment of the CLMs by non-anatomical wedge resections followed by an uneventful clinical course. Histopathological examination of intra-operative liver specimen showed signs consistent with NRH again. 


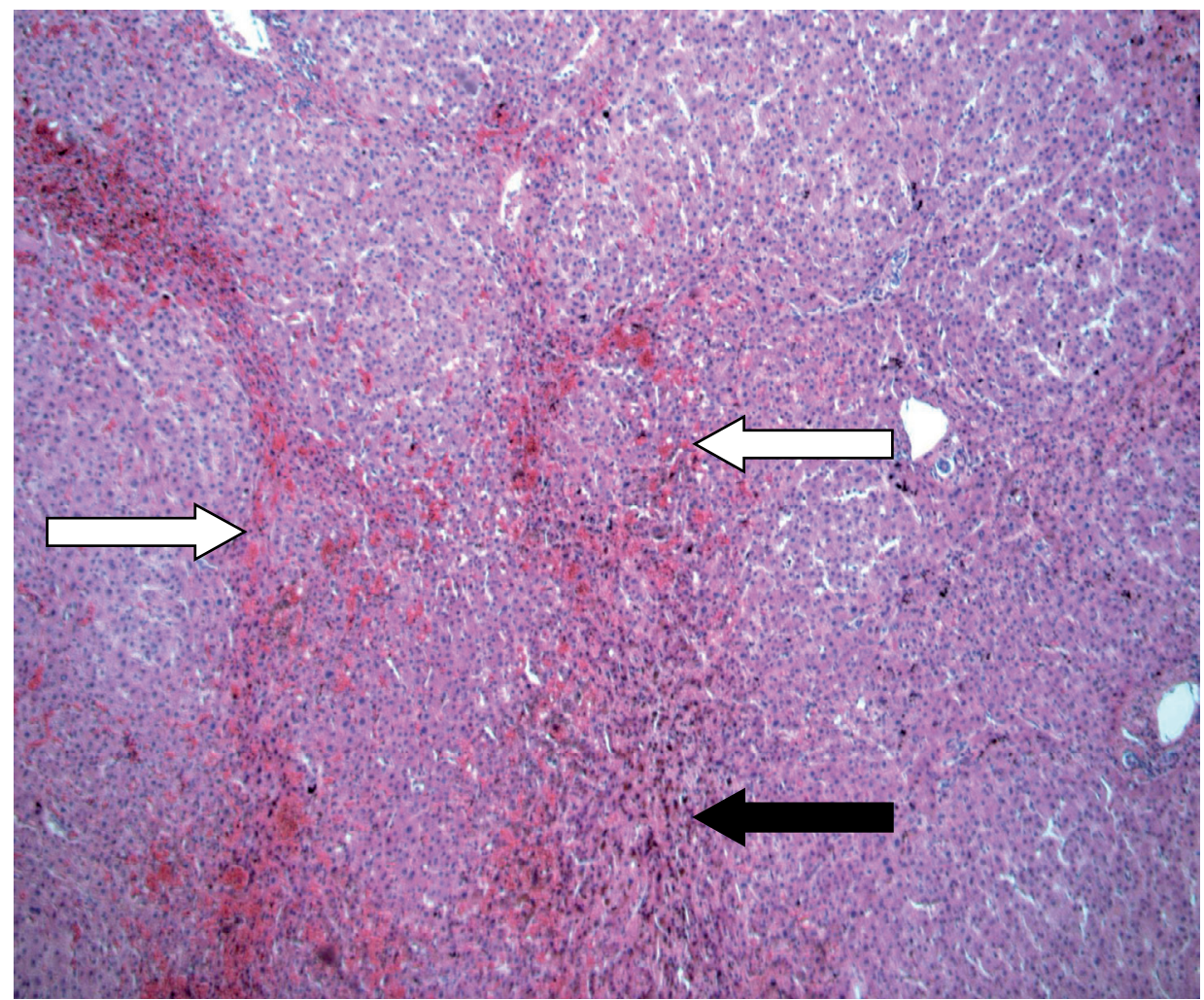

Figure 7.2 Overview of the intra-operatively obtained liver specimen (case 2) in which the liver has a disturbed architecture with nodular appearance of liver parenchyma (white arrows) characteristic of nodular regenerative hyperplasia. Areas with sinusoidal congestion are also present (black arrow). Haematoxylin and eosin, original magnification x 50 .

Eighteen months later, another recurrent CLM was resected from segment IVb after which the clinical course was uneventful. No neoadjuvant chemotherapy was administered prior to surgery this time as the CLM was deemed resectable without the need for downsizing by means of chemotherapy. Histopathological examination of the nontumour-bearing liver did not reveal signs for NRH anymore. However, some areas of the non-tumour-bearing liver did still show minimal sinusoidal congestion.

\section{DISCUSSION}

Chemotherapy is an essential element in the multimodal approach to CLMs. However, chemotherapy consisting of either irinotecan or oxaliplatin has been associated with the development of histological lesions in the non-tumour-bearing liver that are related to post-resectional complications ${ }^{2,6,7}$. Irinotecan has been shown to induce hepatic inflam- 


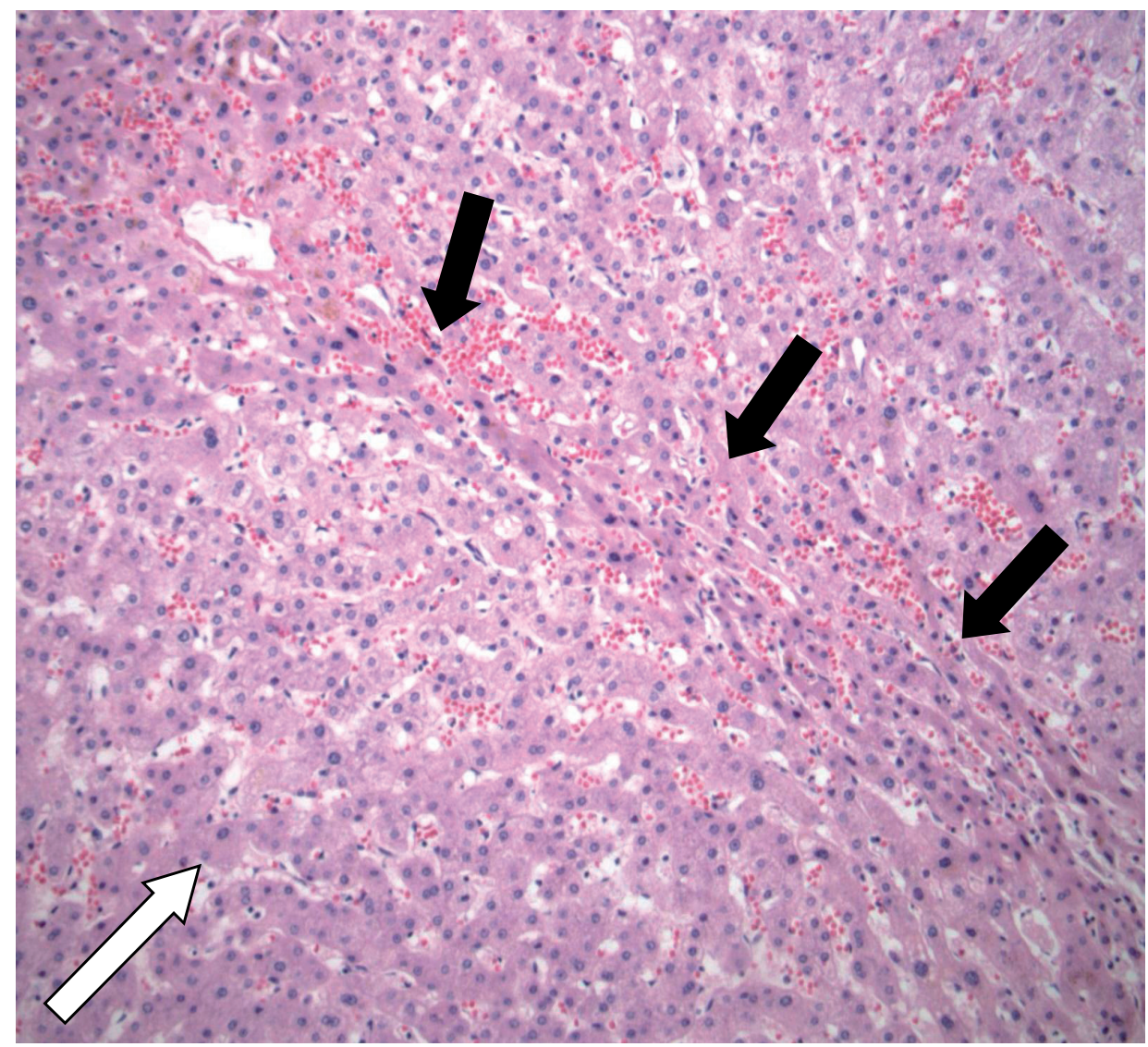

Figure 7.3 Detail of the intra-operative liver specimen (case 2) showing nodular regenerative hyperplasia, in which a regenerative nodule (white arrow) is bordered by irregular aligned, small-sized hepatic trabeculae (black arrows). Haematoxylin and eosin, original magnification $\times 100$.

mation classified as chemotherapy-associated steatohepatitis which is associated with an increased 90 -day mortality after liver surgery ${ }^{6,9}$. Oxaliplatin is related to vascular lesions classified as sinusoidal obstruction syndrome (SOS) and, sporadically, also to $\mathrm{NRH}^{4,5,7,10,11}$. Recently, Nakano and colleagues showed a significant association between the presence of vascular lesions in the non-tumour-bearing liver secondary to oxaliplatin and increased morbidity after major hemihepatectomy?.

$\mathrm{NRH}$ of the liver is characterized by the diffuse presence of regenerative nodules made up of hyperplastic hepatocytes less than $3 \mathrm{~mm}$ in diameter without fibrous septa ${ }^{12}$. The liver parenchyma between the nodules contains atrophic hepatocytes and shows signs of sinusoidal dilatation and congestion (Figure 7.2 and 7.3). Usually, these lesions are clinically asymptomatic but they can be associated with portal hypertension, splenomegaly and bleeding from oesophageal varices ${ }^{13}$. Liver function is usually preserved 
in patients suffering from $\mathrm{NRH}$, although slight increases in alkaline phosphatase or aspatate aminotransferase might be noticed.

The parenchymal injury characteristic of $\mathrm{NRH}$ originates from a heterogeneous perfusion of the liver secondary to obliterative lesions in either the central vein or hepatic sinusoids ${ }^{12}{ }^{14}$. Oxaliplatin-based chemotherapy has a toxic effect on sinusoidal endothelial cells resulting in sinusoidal dilatation, congestion and obstruction. Depletion of sinusoidal glutathione and activation of matrix metalloproteinases by oxaliplatin have been postulated as pathogenic factors in the development of these sinusoidal lesions ${ }^{14}$. Furthermore, it has been hypothesized that the sinusoidal lesions aggravate portal pressure and impair hepatic regeneration ${ }^{5,15}$. The extent of reversibility of these lesions is still uncertain; patients undergoing repeat resection because of recurrent CLMs still showed sinusoidal dilatation or (progressive) fibrosis ${ }^{5}$. On the other hand, a longer interval between neoadjuvant chemotherapy and surgical resection seemed to decrease the incidence of sinusoidal injury ${ }^{7}$.

It is imperative to recognize the presence of SOS or NRH prior to hemihepatectomy. The diagnostic value of a preoperative liver biopsy seems to be minimal because of sampling error resulting in a high false-negative result rate and therefore, suspicion is merely based on the patient's history, preoperative liver function and intra-operative macroscopic aspect. In this respect, several factors independently associated with sinusoidal injury have been identified ${ }^{7}$. These include female gender, administration of 6 or more cycles of oxaliplatin-based chemotherapy, abnormal value of preoperative aspartate aminotransferase (above $36 \mathrm{IU} / \mathrm{L}$ ) and indocyanine green retention rate at 15 minutes of more than 10 per cent. Vauthey and colleagues suggested to perform a preoperative, diagnostic laparoscopy to identify a bluish appearance of the liver ${ }^{6}$.

It could be postulated that, considering the hepatotoxicity of neoadjuvant chemotherapy, initial liver surgery followed by adjuvant chemotherapy might be the treatment of choice in patients with clearly resectable CLMs ${ }^{16}$. However, the effects on diseasefree and long-term survival should be evaluated in adequately powered, randomized controlled trials. In case of unresectable or recurrent CLMs preoperatively treated with oxaliplatin-based chemotherapy, caution is recommended when an extensive hepatic resection is scheduled and SOS or NRH are suspected. Even if the interval between chemotherapeutic treatment and surgical resection is long, SOS or NRH might still be present. Careful analysis of future remnant liver volume and function should guide the operative strategy. Either preoperative portal vein embolization and/or restrictive surgery should be considered when future remnant liver volume or function are regarded insufficient. The safety limit of future remnant liver volume after neoadjuvant chemotherapy treatment would be between 30 and 40 per cent; however, this number needs careful prospective validation. 
Future research should focus on assessment of the optimal duration of chemotherapeutic treatment and timing of liver surgery as well as the development of reliable screening methods for chemotherapy-associated hepatotoxicity and strategies to protect the non-tumour-bearing liver from the deleterious effects of neoadjuvant chemotherapy. 


\section{REFERENCES}

1. Abdalla EK, Adam R, Bilchik AJ, Jaeck D, Vauthey JN, Mahvi D. Improving resectability of hepatic colorectal metastases: expert consensus statement. Ann Surg Oncol 2006; 13: 1271-80.

2. Nordlinger B, Sorbye H, Glimelius B, Poston GJ, Schlag PM, Rougier P, et al. Perioperative chemotherapy with FOLFOX4 and surgery versus surgery alone for resectable liver metastases from colorectal cancer (EORTC Intergroup trial 40983): a randomised controlled trial. Lancet 2008; 371: 1007-16.

3. Adam R, Huguet E, Azoulay D, Castaing D, Kunstlinger F, Levi F, et al. Hepatic resection after downstaging of unresectable hepatic colorectal metastases. Surg Oncol Clin N Am 2003; 12: 211-20, xii.

4. Aloia T, Sebagh M, Plasse M, Karam V, Levi F, Giacchetti S, et al. Liver histology and surgical outcomes after preoperative chemotherapy with fluorouracil plus oxaliplatin in colorectal cancer liver metastases. J Clin Oncol 2006; 24: 4983-90.

5. Rubbia-Brandt L, Audard V, Sartoretti P, Roth AD, Brezault C, Le Charpentier M, et al. Severe hepatic sinusoidal obstruction associated with oxaliplatin-based chemotherapy in patients with metastatic colorectal cancer. Ann Oncol 2004; 15: 460-6.

6. Vauthey JN, Pawlik TM, Ribero D, Wu TT, Zorzi D, Hoff PM, et al. Chemotherapy regimen predicts steatohepatitis and an increase in 90-day mortality after surgery for hepatic colorectal metastases. J Clin Oncol 2006; 24: 2065-72.

7. Nakano H, Oussoultzoglou E, Rosso E, Casnedi S, Chenard-Neu MP, Dufour P, et al. Sinusoidal Injury Increases Morbidity After Major Hepatectomy in Patients With Colorectal Liver Metastases Receiving Preoperative Chemotherapy. Ann Surg 2008; 247: 118-124.

8. Dejong CH, Garden OJ. Neoplasms of the liver. In Majid AA, Kingsnorth A, eds. Advances in surgical practice. London: Greenwich Medical Media, 2003. pp. 146-156.

9. Fong Y, Bentrem DJ. CASH (Chemotherapy-Associated Steatohepatitis) costs. Ann Surg 2006; 243: 8-9.

10. DeLeve LD, Shulman HM, McDonald GB. Toxic injury to hepatic sinusoids: sinusoidal obstruction syndrome (veno-occlusive disease). Semin Liver Dis 2002; 22: 27-42.

11. Washington K, Lane KL, Meyers WC. Nodular regenerative hyperplasia in partial hepatectomy specimens. Am J Surg Pathol 1993; 17: 1151-8.

12. Wanless IR. Micronodular transformation (nodular regenerative hyperplasia) of the liver: a report of 64 cases among 2,500 autopsies and a new classification of benign hepatocellular nodules. Hepatology 1990; 11: 787-97.

13. Reshamwala PA, Kleiner DE, Heller T. Nodular regenerative hyperplasia: not all nodules are created equal. Hepatology 2006; 44: 7-14.

14. DeLeve LD. Hepatic microvasculature in liver injury. Semin Liver Dis 2007; 27: 390-400.

15. Schiffer E, Frossard JL, Rubbia-Brandt L, Mentha G, Pastor CM. Hepatic regeneration is decreased in a rat model of sinusoidal obstruction syndrome. J Surg Oncol 2009; 99: 439-46.

16. Bilchik AJ, Poston G, Curley SA, Strasberg S, Saltz L, Adam R, et al. Neoadjuvant chemotherapy for metastatic colon cancer: a cautionary note. J Clin Oncol 2005; 23: 9073-8. 


\section{Chapter 8}

\section{Hyaluronic acid as a marker of hepatic}

sinusoidal obstruction syndrome secondary to oxaliplatin-based chemotherapy in patients with colorectal liver metastases

Published as:

van den Broek MA, Vreuls CP, Winstanley A, Jansen RL, van Bijnen AA, Dello SA, Bemelmans $\mathrm{MH}$, Dejong $\mathrm{CH}$, Driessen A, Olde Damink SW. Hyaluronic acid as a marker of hepatic sinusoidal obstruction syndrome secondary to oxaliplatin-based chemotherapy in patients with colorectal liver metastases. Ann Surg Oncol 2013: 


\section{ABSTRACT}

\section{Background}

A considerable number of patients develop sinusoidal obstruction syndrome (SOS) after oxaliplatin-based chemotherapy for colorectal liver metastases (CLMs). SOS is associated with adverse outcomes after major hepatectomy. Hyaluronic acid (HA) is a marker of hepatic sinusoidal endothelial cell function and may serve as an accurate marker of SOS. This study aimed to assess the value of systemic HA levels and fractional extraction (FE) of HA by the splanchnic area and liver as markers of SOS after oxaliplatin-based chemotherapy for CLMs.

\section{Methods}

Forty patients were studied. The presence of SOS was assessed histopathologically. Blood samples from the radial artery, portal and hepatic vein were collected. HA levels were determined by ELISA and the FE of HA was estimated.

\section{Results}

SOS was present in 23 patients, of whom 11 showed moderate or severe SOS. Preoperative HA levels were significantly higher in patients with moderate or severe SOS (Group $\mathrm{B}, n=11)$ compared to patients with no or mild SOS (Group $\mathrm{A}, n=29)(51.6 \pm 10.2 \mathrm{ng} / \mathrm{mL}$ versus $32.1 \pm 3.5 \mathrm{ng} / \mathrm{mL}, \mathrm{p}=0.030$ ). A cutoff $\mathrm{HA}$ level of $44.1 \mathrm{ng} / \mathrm{mL}$ yielded a sensitivity of 67 per cent and specificity of 83 per cent for detection of SOS. The positive predictive value was 50 per cent and negative predictive value 91 per cent. Both groups showed a similar FE of HA by the splanchnic area $(-7.9 \pm 8.5$ per cent in Group A versus 7.3 \pm 3.6 per cent in Group $B, p=0.422)$ and liver $(-10.7 \pm 6.2$ per cent in Group A versus $4.6 \pm 2.3$ per cent in Group B, $p=0.265$ ).

\section{Conclusion}

Systemic HA levels can be used to detect patients at risk of SOS after oxaliplatin-based chemotherapy for CLMs. Additional investigations into the presence of SOS are indicated in patients with elevated HA levels. 


\section{INTRODUCTION}

A multidisciplinary approach consisting of chemotherapy followed by surgery is being used increasingly to treat patients with colorectal liver metastases (CLMs). This strategy has proven to enhance resectability rates and increase progression-free survival in resected patients ${ }^{1-3}$. However, treatment with oxaliplatin-based chemotherapy is held responsible for the development of injury to hepatic sinusoidal endothelial cells (SECs), which may manifest as sinusoidal obstruction syndrome (SOS) ${ }^{4,5}$. The presence of SOS has been reported in approximately half of patients treated with oxaliplatin and adversely affects post-resectional outcomes, especially after major liver resection ${ }^{1,5-11}$. In patients scheduled to undergo major liver surgery, information on the presence of SOS prior to surgery is important as it may alter the surgical strategy.

The gold standard for the diagnosis of SOS is measurement of the wedged hepatic venous pressure gradient and assessment of liver pathology ${ }^{12}$. The limitations of obtaining a preoperative liver biopsy are well established, including the risk of needle-track deposits, sampling error, and morbidity associated with the invasive procedure ${ }^{13,14}$. Furthermore, inter- and intraobserver variability in the histopathological assessment of SOS is a problem. Hence, there is a need for a non-invasive, reliable and reproducible screening tool for SOS.

To date, non-invasive detection methods of SOS include elevated ASAT to platelet ratio index (APRI-score), increased spleen size determined by CT volumetry, or increased indocyanine green retention rate. These are all indirect markers of sinusoidal injury and have modest sensitivity and specificity for the diagnosis of $S_{O S}^{6,10,15,16}$. As damage to hepatic SECs is a primary event in the development of SOS, markers for endothelial cell injury may have a better diagnostic accuracy ${ }^{17}$. One of these markers is hyaluronic acid $(\mathrm{HA})^{18-20}$. HA is synthesized by cells of mesodermal lineage. Part of HA turnover occurs by local breakdown. The remainder is transported to the circulation, where approximately 90 per cent is cleared by a receptor-facilitated mechanism almost solely present in hepatic SECs ${ }^{18,21,22}$. Systemic HA levels are thus regarded as a direct marker of hepatic SEC function and have been used for the non-invasive detection of hepatic SEC damage in a variety of clinical conditions ${ }^{18,20,23}$.

When SOS is diagnosed prior to liver surgery, timely adjustments to the operative plan can be made. Strategies such as portal vein occlusion and/or two-stage hepatectomy can be applied to enhance or preserve future remnant liver volume and function ${ }^{15,24}$. This study aimed to assess the value of systemic HA levels and fractional extraction (FE) of HA by the splanchnic area and liver as markers of SOS after oxaliplatin-based chemotherapy in patients with CLMs. 


\section{METHODS}

\section{Patient selection}

Consecutive patients undergoing partial hepatectomy for CLMs at Maastricht University Medical Centre between January 2008 and December 2009 were prospectively included. Exclusion criteria were (a) laparoscopic liver resection, (b) no oxaliplatin-based chemotherapy or interval between chemotherapy and liver surgery more than 12 months, (c) repeat liver surgery, (d) liver tissue or plasma not suitable for analysis, and (e) presence of inflammatory joint disease. The study was performed in accordance with the ethical standards of the Declaration of Helsinki.

\section{Protocols of chemotherapy}

Patients presenting with CLMs were discussed in our multidisciplinary oncology team. On indication, patients received bevacizumab as $7.5 \mathrm{mg} / \mathrm{kg}$ and oxaliplatin as $130 \mathrm{mg} / \mathrm{m}^{2}$ intravenous infusion on day 1 combined with oral capecitabine two-times daily dosed $1000 \mathrm{mg} / \mathrm{m}^{2}$ on day 1-14, followed by a treatment-free week (day 15-21). After 3 cycles, eligibility for and optimal timing of surgery was determined based on the radiological tumour response ${ }^{25}$. The waiting time between end of chemotherapy and surgery was kept at a minimum of 4 weeks.

\section{Preoperative management}

Standard laboratory tests were obtained preoperatively from the clinical chemistry laboratory. The APRI-score was calculated using the following formula: APRI-score = [(ASAT / upper limit of normal of ASAT) / platelet count $]^{*} 100$ per cent ${ }^{26}$. The upper limit of normal of ASAT was 30 IU/L. Data on patient demographics, extent of liver disease, chemotherapy regimens, and interval between chemotherapy and surgery were collected.

\section{Surgical procedure}

Surgical procedures were performed as described before ${ }^{27}$. In short, patients routinely had indwelling catheters in a jugular vein and radial artery. After liver mobilization, an ultrasound was performed which guided the operative strategy. A Cavitron Ultrasonic Surgical Aspirator (CUSA system 200 macrodissector, Cavitron Surgical Systems, Stamford, CT, USA) and Argon beam coagulation (Force GSU System, Valleylab, Boulder, CO, USA) were used for liver transection.

\section{Postoperative care}

The postoperative care was according to an enhanced recovery after liver surgery programme ${ }^{27}$. Liver surgery-related complications were recorded prospectively. These 
included bile leakage, ascites, post-resectional liver failure, intra-abdominal hemorrhage, intra-abdominal abscess and operative mortality with a Clavien-Dindo grade of 3 or more and occurring within 90 days after surgery ${ }^{28,29}$.

\section{Blood sampling}

Blood was sampled from the radial artery before the start of surgery. In a subgroup, additional samples were collected intra-operatively from the radial artery, portal vein and hepatic vein as described in ${ }^{30}$. Blood samples were transferred to prechilled heparincoated blood collection tubes and put on ice. Plasma was prepared by centrifugation at $4^{\circ} \mathrm{C}$ at $3500 \times \mathrm{g}$ for $15 \mathrm{~min}$ after which supernatant was stored in aliquots at $-80^{\circ} \mathrm{C}$ till analysis.

\section{Tissue sampling and histopathological analysis}

After resection, the specimen was sliced and fixed in 4 per cent buffered formaldehyde. Tissue samples were taken from the non-tumour-bearing liver at a minimum distance of $2 \mathrm{~cm}$ from the tumour while avoiding the subcapsular region. They were routinely embedded in paraffin and $4 \mu \mathrm{m}$ sections were cut. Sections were examined by two experienced HPB pathologists unaware of the clinical data. In case of discrepancy, the section was discussed and consensus was reached. Morphological analysis was based on haematoxylin and eosin, reticulin and Trichrome Masson stains. Histopathological features containing vascular and parenchymal changes were scored according to the classification of Rubbia-Brandt and colleagues ${ }^{5}$. Sinusoidal dilatation was graded as follows: grade 0. no dilatation, grade 1. centrilobular dilatation in zone 3 [mild SOS], grade 2. centrilobular dilatation in zones 2 and 3 [moderate SOS], and grade 3. panlobular sinusoidal dilatation and bridging congestion [severe SOS]. For analysis, two groups were created: group A consisted of no lesions and grade 1 lesions and group B of grade 2 and 3 lesions, as described earlier in ${ }^{6,11}$. Peliosis and nodular regenerative hyperplasia (NRH) were scored as present or absent.

\section{Systemic hyaluronic acid level as a marker of SOS}

HA levels were determined using a commercially available ELISA kit (Corgenix Inc., Broomfield, CO, USA), which had a lower limit of detection of $10 \mathrm{ng} / \mathrm{mL}$. According to the manufacturer, HA levels in a healthy population ranged between $0-75 \mathrm{ng} / \mathrm{mL}$. Samples below the detection limit were excluded.

\section{Fractional extraction of hyaluronic acid}

$\mathrm{FE}$ of $\mathrm{HA}$ by the splanchnic area $\left(\mathrm{FE}_{\text {splanchnic }}\right)$ and liver $\left(\mathrm{FE}_{\text {liver }}\right)$ was calculated using the following formulas: $\mathrm{FE}_{\text {splanchnic }}=[$ (hepatic vein level - radial artery level) / radial artery level $]^{*} 100$ per cent and $\mathrm{FE}_{\text {liver }}=\left[\right.$ (hepatic vein level $-\left(0.3^{*}\right.$ radial artery level $+0.7^{*}$ portal 
vein level $)) /\left(0.3^{*}\right.$ radial artery level $+0.7^{*}$ portal vein level $){ }^{*} 100$ per cent. The $\mathrm{FE}_{\text {liver }}$ represents the percentage of $\mathrm{HA}$ that is actually taken up by the liver in relation to its inflow. Since we were unable to measure portal vein and hepatic artery flow, we assumed that approximately 30 per cent of hepatic inflow was derived from the hepatic artery and 70 per cent from the portal vein $\left(\right.$ see $\left.^{31}\right)$. Positive values represent net release and negative values net uptake.

\section{Statistical analysis}

Data are given as mean with standard error or median with range. For continuous data, statistical analyses were performed using a student $t$ test or Mann-Whitney $U$ test, depending on the nature of the data. Differences between dichotomous variables were calculated with Pearson's chi-square test. The FE of HA was tested using a one-sample t-test with a theoretical mean of zero. A receiver operating characteristic curve was created to determine the clinical performance and optimal diagnostic cutoff value of systemic HA levels. Analyses were performed using SPSS version 15.0 for Windows (SPSS Inc. Chicago, IL, USA). A p-value below 0.050 was considered significant.

\section{RESULTS}

\section{Patient characteristics}

During the study period, 108 patients underwent a partial hepatectomy, of whom 40 were eligible for inclusion (Figure 8.1). Their characteristics are described in Table 8.1. The median number of oxaliplatin cycles was 5 (2-10) with a median interval between oxaliplatin and surgery of 61 days (26-338 days). In 35 patients (88 per cent), bevacizumab was added to oxaliplatin.

\section{Surgical procedure}

The operative strategy was a liver-first approach in 9, a conventional approach in 30, and a two-stage approach in 1 patient, respectively ${ }^{32}$. A median of 3 liver segments (range 1-5) was resected. Additional operative characteristics are depicted in Table 8.2.

\section{Incidence of hepatic SOS}

SOS was present in 23 patients (58 per cent). Mild SOS was seen in 12 (30 per cent), moderate in 9 ( 23 per cent) and severe in 2 patients ( 5 per cent), respectively. Furthermore, 2 patients ( 5 per cent) showed peliosis, both with the presence of moderate or severe SOS. Patients with different SOS grade were divided into group A, consisting of 29 patients with no or mild sinusoidal dilatation, and group B, consisting of 11 patients with moderate or severe sinusoidal dilatation. There was no difference between group $A$ and 


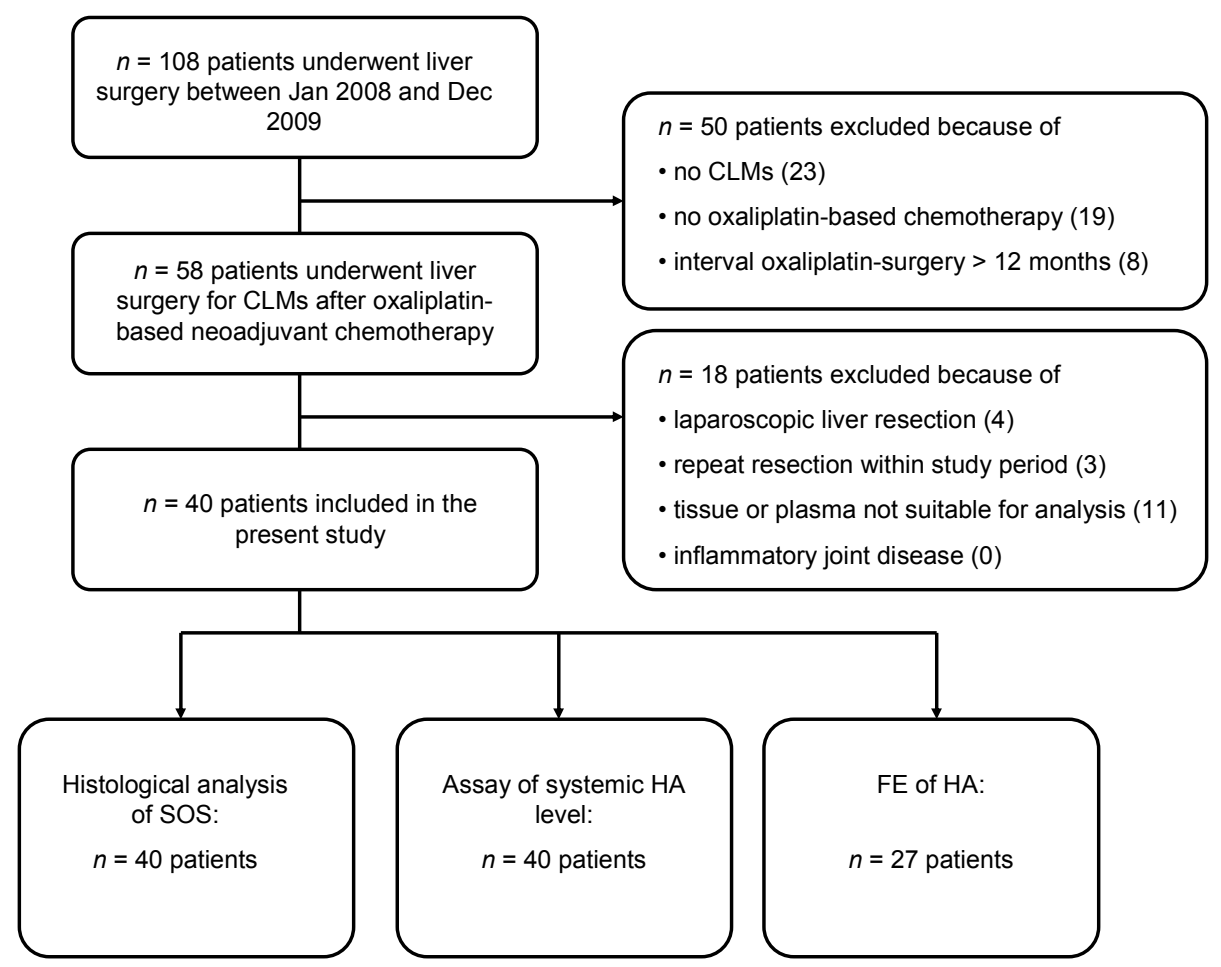

Figure 8.1 Flowchart of patients.

Legend: CLM, colorectal liver metastasis; HA, hyaluronic acid; SOS, sinusoidal obstruction syndrome; FE, fractional extraction.

Table 8.1 Patient characteristics.

\section{$N=\mathbf{4 0}$}

\section{Baseline characteristics}

Age (years)

$61 \pm 2$

Sex (male)

$20(50)$

BMI $\left(\mathrm{kg} / \mathrm{m}^{2}\right)$

$26 \pm 1$

ASA classification

$$
\text { ASA I }
$$

ASA II

ASA III

Primary disease

Colon

Rectum

Colorectal liver metastases

Synchronous

Metachronous 
Table 8.1 Continued

\begin{tabular}{|c|c|}
\hline Recurrent & $3(8)$ \\
\hline Median no. of metastases (range) & $2(1-10)$ \\
\hline Size of largest hepatic metastases (mm) & $23 \pm 3$ \\
\hline Presence of extra-hepatic disease & $5(13)$ \\
\hline \multicolumn{2}{|l|}{ Preoperative liver function } \\
\hline ASAT (IU/L) & $27 \pm 2$ \\
\hline ALAT (IU/L) & $32 \pm 2$ \\
\hline Total bilirubin $(\mu \mathrm{mol} / \mathrm{L})$ & $14 \pm 1$ \\
\hline INR & $1 \pm 0$ \\
\hline APRI-score & $0.46 \pm 0.05$ \\
\hline \multicolumn{2}{|l|}{ Chemotherapy regimens } \\
\hline CAPOX & $5(13)$ \\
\hline CAPOX + bevacizumab & $32(80)$ \\
\hline CAPOX + CAPIRI + bevacizumab & $3(8)$ \\
\hline \multicolumn{2}{|l|}{ Amount of chemotherapy } \\
\hline \multicolumn{2}{|l|}{ Oxaliplatin } \\
\hline - Median no. of cycles (range) & $5(2-10)$ \\
\hline - Median cumulative dose $\left(\mathrm{mg} / \mathrm{m}^{2}\right)$ (range) & $650(240-1300)$ \\
\hline - Median interval chemotherapy - surgery (days) & $61(26-338)$ \\
\hline \multicolumn{2}{|l|}{ Bevacizumab } \\
\hline - Median no. of cycles (range) & $3(1-7)$ \\
\hline - Median cumulative dose (mg/kg) (range) & $22.5(7.5-52.5)$ \\
\hline - Median interval chemotherapy - surgery (days) & $79(26-359)$ \\
\hline \multicolumn{2}{|l|}{ Irinotecan } \\
\hline - Median no. of cycles (range) & $3(1-6)$ \\
\hline - Median cumulative dose $\left(\mathrm{mg} / \mathrm{m}^{2}\right)$ (range) & $1050(350-2800)$ \\
\hline - Median interval chemotherapy - surgery (days) & $129(67-364)$ \\
\hline
\end{tabular}

Numbers indicate mean \pm standard error or absolute number (percentage) unless otherwise indicated; $N$ and no., number; ASA, American Society of Anaesthesiologists; BMI, body mass index; ASAT, aspartate aminotransferase; ALAT, alanine aminotransferase; INR, international standardized ratio; APRI-score, ASAT to platelet ratio index; CAPOX, capecitabine + oxaliplatin; CAPIRI, capecitabine + irinotecan.

group B with respect to number of oxaliplatin cycles, interval between oxaliplatin and surgery or addition of bevacizumab (Table 8.3). Also APRI-scores, which were available in 16 patients, were comparable between the groups. In addition, there was no relation between the presence of SOS and adverse post-resectional outcome.

\section{Systemic hyaluronic acid level as a marker of SOS}

Mean systemic HA levels before the start of surgery were significantly higher in patients with moderate or severe SOS compared to patients with no or mild SOS $(51.6 \pm 10.2 \mathrm{ng} /$ 
Table 8.2 Operative characteristics and pathological findings.

\begin{tabular}{|c|c|}
\hline & $N=40$ \\
\hline \multicolumn{2}{|l|}{ Surgery } \\
\hline \multicolumn{2}{|l|}{ Type of resection ${ }^{\#}$} \\
\hline Hemihepatectomy & $15(38)$ \\
\hline Central bisectionectomy & $5(13)$ \\
\hline Bisegmentectomy & $4(10)$ \\
\hline Unisegmentectomy & $4(10)$ \\
\hline Non-anatomical resection & $12(30)$ \\
\hline Median no. of resected segments (range) & $3(1-5)$ \\
\hline \multicolumn{2}{|l|}{ Adjuvant techniques } \\
\hline Portal vein ligation & $3(8)$ \\
\hline Two-stage hepatectomy & $1(3)$ \\
\hline Median blood loss (mL) (range) & $750(200-11000)$ \\
\hline Red blood cell transfusion & $12(30)$ \\
\hline Duration of surgery (min) & $252 \pm 14$ \\
\hline \multicolumn{2}{|l|}{ Pathology* } \\
\hline \multicolumn{2}{|l|}{ Sinusoidal dilatation } \\
\hline No & $17(43)$ \\
\hline Mild (grade 1) & $12(30)$ \\
\hline Moderate (grade 2) & $9(23)$ \\
\hline Severe (grade 3) & $2(5)$ \\
\hline Peliosis & $2(5)$ \\
\hline Nodular regenerative hyperplasia & $11(28)$ \\
\hline
\end{tabular}

Numbers indicate mean \pm standard error or absolute number (percentage) unless otherwise indicated; \# According to the IHPBA nomenclature ${ }^{44}$; ${ }^{*}$ graded according to Rubbia-Brandt and colleagues ${ }^{5}$; N, number.

Table 8.3 Comparative analysis of baseline, operative and postoperative characteristics between patients with no or mild SOS (Group A) and moderate or severe SOS (Group B).

\begin{tabular}{lccc}
\hline & Group A & Group B & p-value \\
& $n=29$ & $n=11$ & 0.463 \\
\hline Baseline characteristics & & & 0.288 \\
Age (years) & $62 \pm 2$ & $59 \pm 3$ & 0.270 \\
Sex (male) & $13(45)$ & $7(64)$ & 0.751 \\
BMI (kg/m ${ }^{2}$ ) & $25 \pm 1$ & $27 \pm 1$ & 0.974 \\
Median no. of CLMs & $2(1-10)$ & $2(1-5)$ & 0.538 \\
Size of largest CLM (mm) & $23 \pm 4$ & $22 \pm 5$ & $0(0)$ \\
Presence of extra-hepatic disease & $5(17)$ & & 0.177 \\
Preoperative liver function & & $23 \pm 4$ & 0.092 \\
ASAT (IU/L) & $29 \pm 2$ & $25 \pm 5$ & \\
ALAT (IU/L) & $34 \pm 3$ & & \\
\hline
\end{tabular}


Table 8.3 Continued

\begin{tabular}{|c|c|c|c|}
\hline & Group A & Group B & p-value \\
\hline Bilirubin ( $\mu \mathrm{mol} / \mathrm{L})$ & $13 \pm 1$ & $15 \pm 1$ & 0.257 \\
\hline INR & $1 \pm 0$ & $1 \pm 0$ & 0.860 \\
\hline \multicolumn{4}{|l|}{ Markers of SOS } \\
\hline Hyaluronic acid (ng/mL) & $32.1 \pm 3.5$ & $51.6 \pm 10.2$ & 0.030 \\
\hline APRI-score* & $0.42 \pm 0.07$ & $0.61 \pm 0.18$ & 0.254 \\
\hline \multicolumn{4}{|l|}{ Fractional extraction of hyaluronic acid } \\
\hline Splanchnic area (\%) & $-7.9 \pm 8.5$ & $7.3 \pm 3.6$ & 0.422 \\
\hline Liver (\%) & $-10.7 \pm 6.2$ & $4.6 \pm 2.3$ & 0.265 \\
\hline \multicolumn{4}{|l|}{ Chemotherapy regimens } \\
\hline Bevacizumab-containing regimen & $26(90)$ & $9(82)$ & 0.503 \\
\hline Median no. cycles oxaliplatin & $5(3-9)$ & $5(2-10)$ & 0.580 \\
\hline Median interval oxaliplatin (days) & $61(26-338)$ & $61(39-253)$ & 0.940 \\
\hline \multicolumn{4}{|l|}{ Surgery } \\
\hline Median no. resected segments & $3(1-5)$ & $3(1-4)$ & 0.704 \\
\hline Median blood loss (mL) & $700(200-11000)$ & $900(200-2200)$ & 0.693 \\
\hline Duration of surgery (min) & $249 \pm 16$ & $258 \pm 27$ & 0.775 \\
\hline \multicolumn{4}{|l|}{ Postoperative outcome } \\
\hline Liver surgery-related complications & $8(28)$ & $3(27)$ & 0.984 \\
\hline Operative mortality & $2(7)$ & $0(0)$ & \\
\hline Bile leakage & $5(17)$ & $2(18)$ & \\
\hline Ascites & $2(7)$ & $0(0)$ & \\
\hline Post-resectional liver failure & $1(3)$ & $0(0)$ & \\
\hline Intra-abdominal abscess & $6(21)$ & $2(18)$ & \\
\hline Intra-abdominal hemorrhage & $0(0)$ & $0(0)$ & \\
\hline Median length of stay (days) & $8(5-33)$ & $8(5-38)$ & 0.329 \\
\hline
\end{tabular}

Numbers indicate mean \pm standard error or absolute number (percentage) unless otherwise indicated; $N$, number; BMI, body mass index; CLM, colorectal liver metastasis; SOS, sinusoidal obstruction syndrome; ASAT, aspartate aminotransferase; ALAT, alanine aminotransferase; INR, international standardized ratio; APRI-score, ASAT to platelet ratio index; * only available in 16 patients.

$\mathrm{mL}$ versus $32.1 \pm 3.5 \mathrm{ng} / \mathrm{mL}, \mathrm{p}=0.030$ ). The area under the curve of systemic HA levels for identification of SOS was 0.736 (95 per cent confidence interval 0.490-0.992, $\mathrm{p}=0.078$ ). An optimal cutoff value of $44.1 \mathrm{ng} / \mathrm{mL}$ yielded a sensitivity of 67 per cent and specificity of 83 per cent for detection of moderate or severe SOS. The positive predictive value of this level was 50 per cent and negative predictive value 91 per cent.

\section{Fractional extraction of hyaluronic acid}

In 27 patients, intra-operative samples for the calculation of the FE of HA were available. Mean HA levels in the radial artery, portal and hepatic vein did not differ significantly 
between Group A and B at this time point (level of significance not shown) (Figure 8.2). The $\mathrm{FE}_{\text {splanchnic }}$ of $\mathrm{HA}$ was comparable between Group $\mathrm{A}$ and $\mathrm{B}(-7.9 \pm 8.5$ per cent versus 7.3 \pm 3.6 per cent, $p=0.422$ ). The $\mathrm{FE}_{\text {liver }}$ of $\mathrm{HA}$ was $-10.7 \pm 6.2$ per cent in patients Group $A$, representing a trend towards net hepatic uptake ( $p=0.087$ from zero). In patients in Group $B, \mathrm{FE}_{\text {liver }}$ of $\mathrm{HA}$ was $4.6 \pm 2.3$ per cent, representing a non-significant net hepatic release ( $p=0.176$ from zero). The difference in $\mathrm{FE}_{\text {liver }}$ did also not reach statistical significance $(p=0.265)$.

\section{DISCUSSION}

In the present study, fifty-eight per cent of patients showed histological evidence of SOS. Systemic HA levels were significantly elevated in patients with moderate or severe SOS compared to patients with no or mild SOS. The diagnostic accuracy of systemic HA levels for the detection of SOS was fair. Although there seemed to be a trend towards net hepatic uptake in patients with no or mild SOS versus net hepatic release in patients with moderate or severe SOS, the FE of HA was not significantly different between these groups.

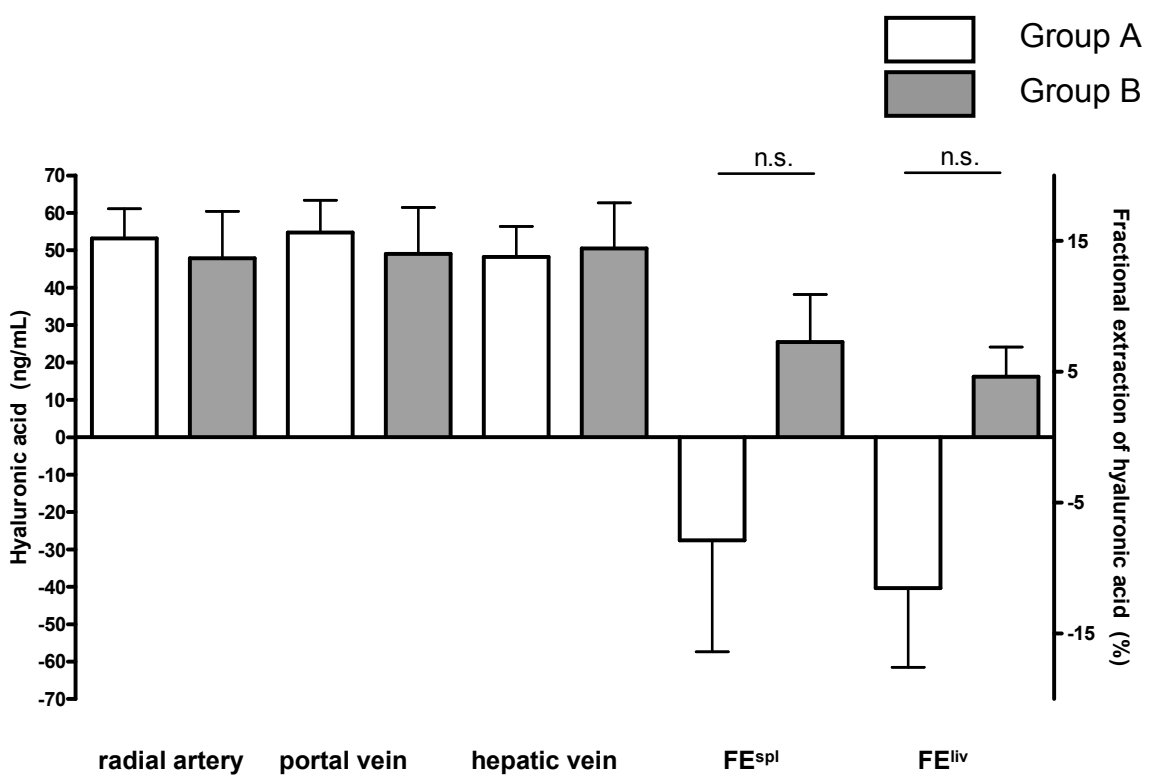

Figure 8.2 Intra-operative HA levels in the radial artery, portal vein and hepatic vein and fractional extraction of HA by the splanchnic area and liver in patients with no or mild SOS (Group A) and with moderate or severe SOS (Group B).

Legend: $\mathrm{HA}$, hyaluronic acid; $\mathrm{FE}_{\text {spl' }}$ fractional extraction by the splanchnic area; $\mathrm{FE}_{\text {liv' }}$ fractional extraction by the liver; SOS, sinusoidal obstruction syndrome; n.s., not significant. 
The prevalence of SOS in our cohort was in the same range as data reported by other groups $^{5-8,10,33}$. Surprisingly, we found no association between the presence of SOS and the number of oxaliplatin cycles, interval between oxaliplatin and surgery or addition of bevacizumab $5,6,10$. This may either be explained by an inter-individual variation in susceptibility to developing SOS or the small number of patients included in the present study.

To the best of our knowledge, this is the first study analyzing the value of systemic $\mathrm{HA}$ levels for the detection of SOS in patients with CLMs. Observed HA levels, although significantly elevated in patients with SOS, were within the normal range as was the optimal cutoff level for identification of patients with advanced SOS. These results may indicate that SEC injury induced by oxaliplatin was less severe than injury induced by other toxic substances, such as myeloablative conditioning therapy. In the latter condition, patients show HA levels which are on average tenfold higher than the upper limit of normal ${ }^{20}$.

Increased systemic HA levels can either be due to decreased extraction or to increased production. Although there seemed to be a trend towards net uptake in patients with no or mild SOS versus net release in patients with moderate or severe SOS by the splanchnic area and liver, our attempt to estimate the FE of HA was not successful in detecting a significant difference. The reason for this remains speculative, but this observation may be due to the large variation in the $\mathrm{FE}$, making our study underpowered to detect a significant difference. Otherwise, our assumption of the 30 per cent versus 70 per cent distribution of inflow may be incorrect, though FE by the splanchnic area, which does not rely on this assumption, showed the same trend.

The comparable FE of HA may as well reflect the actual fact that the net functional capacity of hepatic SECs for extraction of HA was not disturbed at the time of liver surgery, despite histological evidence of SOS. This could be related to (1) a recovery of SEC function during the interval between chemotherapy and surgery or (2) an altered intrahepatic blood flow secondary to sinusoidal injury, which is typically observed in patients suffering from $\mathrm{NRH}^{5,34,35}$. In patients with $\mathrm{NRH}$, regions with hyperplastic hepatocytes alternate regions with hypoplastic hepatocytes as a result of a heterogenous perfusion secondary to SEC injury. Furthermore, our data point out that an increased release of HA by damaged SECs or enhanced production by hepatic stellate cells, which become activated in advanced SOS, is unlikely ${ }^{36-38}$. Taken together, the increased systemic HA levels in patients with SOS do not result from decreased hepatic uptake or increased hepatic release and may be explained by either portosystemic shunting due to portal hypertension or enhanced production at other sites than the liver. From a mechanistic point of view, it would be relevant to obtain more detailed insight into HA metabolism and SEC function in patients with SOS after oxaliplatin-based chemotherapy by either performing HA loading tests or PET scans with $\left[{ }^{11} \mathrm{C}\right]$ hyaluronic acid ${ }^{39,40}$. 
Other methods to predict SOS use indirect markers of SEC injury and may therefore reflect other disease states as well ${ }^{6,10,11}$. The APRI-scores did not differ significantly in the present study, but, as preoperative platelet counts were not available in the majority of patients, the diagnostic accuracy of HA could not be compared to that of the APRI-score. It is likely that a combination of biomarkers comprising genetic, functional, radiological and damage parameters would be required to accurately stratify patients ${ }^{6,16}$. Circulating von Willebrand Factor seems a reasonable candidate marker based on recent pathophysiological insights derived from gene array studies in humans ${ }^{41-43}$.

Our study has some limitations. The accuracy of the HA assay was moderate due to its relatively high lower limit of detection. Optimization of the assay protocol and validation of our results in a larger patient cohort are warranted. In addition, systemic HA levels were measured at the start of surgery only. Consequently, information is lacking on the direct toxic effect of oxaliplatin on hepatic SECs, taken into account the 60 day interval between chemotherapy and surgery. Serial sampling just before and after chemotherapy might shed a light on temporary SEC dysfunction after oxaliplatin. Finally, the assay of systemic HA levels yielded a high specificity and negative predictive value at the cutoff level of $44.1 \mathrm{ng} / \mathrm{mL}$ for the diagnosis of moderate or severe SOS. This implies that patients with low HA levels most probably do not suffer from sinusoidal injury. In patients with high HA levels, additional investigations are warranted.

To conclude, elevated systemic HA levels had a fair diagnostic performance for the detection of patients with moderate or severe SOS after oxaliplatin-based chemotherapy for CLMs. In patients with high HA levels scheduled to undergo major hepatectomy, preoperative assessment of liver pathology by a transjular liver biopsy and wedged hepatic venous pressure gradient measurement may be indicated in order to identify those who would benefit most from two-stage hepatectomy with portal vein occlusion. 


\section{REFERENCES}

1. Nordlinger B, Sorbye H, Glimelius B, Poston GJ, Schlag PM, Rougier P, et al. Perioperative chemotherapy with FOLFOX4 and surgery versus surgery alone for resectable liver metastases from colorectal cancer (EORTC Intergroup trial 40983): a randomised controlled trial. Lancet 2008; 371: 1007-16.

2. Abdalla EK, Adam R, Bilchik AJ, Jaeck D, Vauthey JN, Mahvi D. Improving resectability of hepatic colorectal metastases: expert consensus statement. Ann Surg Oncol 2006; 13: 1271-80.

3. Lam VW, Spiro C, Laurence JM, Johnston E, Hollands MJ, Pleass HC, et al. A Systematic Review of Clinical Response and Survival Outcomes of Downsizing Systemic Chemotherapy and Rescue Liver Surgery in Patients with Initially Unresectable Colorectal Liver Metastases. Ann Surg Oncol 2011; 19: 1292-301.

4. DeLeve LD. Hepatic microvasculature in liver injury. Semin Liver Dis 2007; 27: 390-400.

5. Rubbia-Brandt L, Lauwers GY, Wang H, Majno PE, Tanabe K, Zhu AX, et al. Sinusoidal obstruction syndrome and nodular regenerative hyperplasia are frequent oxaliplatin-associated liver lesions and partially prevented by bevacizumab in patients with hepatic colorectal metastasis. Histopathology 2010; 56: 430-9.

6. Soubrane $\mathrm{O}$, Brouquet A, Zalinski S, Terris B, Brezault C, Mallet V, et al. Predicting high grade lesions of sinusoidal obstruction syndrome related to oxaliplatin-based chemotherapy for colorectal liver metastases: correlation with post-hepatectomy outcome. Ann Surg 2010; 251: 454-60.

7. Aloia T, Sebagh M, Plasse M, Karam V, Levi F, Giacchetti S, et al. Liver histology and surgical outcomes after preoperative chemotherapy with fluorouracil plus oxaliplatin in colorectal cancer liver metastases. J Clin Oncol 2006; 24: 4983-90.

8. Karoui M, Penna C, Amin-Hashem M, Mitry E, Benoist S, Franc B, et al. Influence of preoperative chemotherapy on the risk of major hepatectomy for colorectal liver metastases. Ann Surg 2006; 243: 1-7.

9. Vauthey JN, Pawlik TM, Ribero D, Wu TT, Zorzi D, Hoff PM, et al. Chemotherapy regimen predicts steatohepatitis and an increase in 90-day mortality after surgery for hepatic colorectal metastases. J Clin Oncol 2006; 24: 2065-72.

10. Nakano H, Oussoultzoglou E, Rosso E, Casnedi S, Chenard-Neu MP, Dufour P, et al. Sinusoidal Injury Increases Morbidity After Major Hepatectomy in Patients With Colorectal Liver Metastases Receiving Preoperative Chemotherapy. Ann Surg 2008; 247: 118-124.

11. Tamandl D, Klinger M, Eipeldauer S, Herberger B, Kaczirek K, Gruenberger B, et al. Sinusoidal obstruction syndrome impairs long-term outcome of colorectal liver metastases treated with resection after neoadjuvant chemotherapy. Ann Surg Oncol 2010; 18: 421-30.

12. DeLeve LD, Valla DC, Garcia-Tsao G. Vascular disorders of the liver. Hepatology 2009; 49: 1729-64.

13. Bravo AA, Sheth SG, Chopra S. Liver biopsy. N Engl J Med 2001; 344: 495-500.

14. Jones OM, Rees M, John TG, Bygrave S, Plant G. Biopsy of resectable colorectal liver metastases causes tumour dissemination and adversely affects survival after liver resection. Br J Surg 2005; 92: 1165-8.

15. Narita M, Oussoultzoglou E, Fuchshuber P, Pessaux P, Chenard MP, Rosso E, et al. What Is a Safe Future Liver Remnant Size in Patients Undergoing Major Hepatectomy for Colorectal Liver Metastases and Treated by Intensive Preoperative Chemotherapy? Ann Surg Oncol 2012; 19: 2526-38.

16. Overman MJ, Maru DM, Charnsangavej C, Loyer EM, Wang H, Pathak P, et al. Oxaliplatin-mediated increase in spleen size as a biomarker for the development of hepatic sinusoidal injury. $J$ Clin Oncol 2010; 28: 2549-55. 
17. DeLeve LD, McCuskey RS, Wang X, Hu L, McCuskey MK, Epstein RB, et al. Characterization of a reproducible rat model of hepatic veno-occlusive disease. Hepatology 1999; 29: 1779-91.

18. Fraser JR, Laurent TC, Laurent UB. Hyaluronan: its nature, distribution, functions and turnover. $J$ Intern Med 1997; 242: 27-33.

19. Lindqvist $U$. Is serum hyaluronan a helpful tool in the management of patients with liver diseases? J Intern Med 1997; 242: 67-71.

20. Fried MW, Duncan A, Soroka S, Connaghan DG, Farrand A, Peter J, et al. Serum hyaluronic acid in patients with veno-occlusive disease following bone marrow transplantation. Bone Marrow Transplant 2001; 27: 635-9.

21. Eriksson S, Fraser JR, Laurent TC, Pertoft H, Smedsrod B. Endothelial cells are a site of uptake and degradation of hyaluronic acid in the liver. Exp Cell Res 1983; 144: 223-8.

22. Elvevold K, Smedsrod B, Martinez I. The liver sinusoidal endothelial cell: a cell type of controversial and confusing identity. Am J Physiol Gastrointest Liver Physiol 2008; 294: G391-400.

23. Bronsther OL, Rao PN, Pinna A, Snyder J, Cowan S, Kramer D, et al. Effluent levels of hyaluronic acid can predict ultimate graft outcome after clinical liver transplantation: a prospective series. Transplant Proc 1993; 25: 1538-40.

24. Chun YS, Laurent A, Maru D, Vauthey JN. Management of chemotherapy-associated hepatotoxicity in colorectal liver metastases. Lancet Oncol 2009; 10: 278-86.

25. Therasse P, Arbuck SG, Eisenhauer EA, Wanders J, Kaplan RS, Rubinstein L, et al. New guidelines to evaluate the response to treatment in solid tumors. European Organization for Research and Treatment of Cancer, National Cancer Institute of the United States, National Cancer Institute of Canada. J Natl Cancer Inst 2000; 92: 205-16.

26. Wai CT, Greenson JK, Fontana RJ, Kalbfleisch JD, Marrero JA, Conjeevaram HS, et al. A simple noninvasive index can predict both significant fibrosis and cirrhosis in patients with chronic hepatitis C. Hepatology 2003; 38: 518-26.

27. van Dam RM, Hendry PO, Coolsen MM, Bemelmans MH, Lassen K, Revhaug A, et al. Initial experience with a multimodal enhanced recovery programme in patients undergoing liver resection. $\mathrm{Br}$ J Surg 2008; 95: 969-75.

28. van den Broek MA, van Dam RM, van Breukelen GJ, Bemelmans MH, Oussoultzoglou E, Pessaux $P$, et al. Development of a composite endpoint for randomized controlled trials in liver surgery. $\mathrm{Br} J$ Surg 2011; 98: 1138-45.

29. Dindo D, Demartines N, Clavien PA. Classification of surgical complications: a new proposal with evaluation in a cohort of 6336 patients and results of a survey. Ann Surg 2004; 240: 205-13.

30. van den Broek MA, Bloemen JG, Dello SA, van de Poll MC, Olde Damink SW, Dejong CH. Randomized controlled trial analyzing the effect of 15 or 30 min intermittent Pringle maneuver on hepatocellular damage during liver surgery. $J$ Hepatol 2011; 55: 337-45.

31. van de Poll MC, Siroen MP, van Leeuwen PA, Soeters PB, Melis GC, Boelens PG, et al. Interorgan amino acid exchange in humans: consequences for arginine and citrulline metabolism. Am J Clin Nutr 2007; 85: 167-72.

32. de Jong MC, van Dam RM, Maas M, Bemelmans MH, Olde Damink SW, Beets GL, et al. The liverfirst approach for synchronous colorectal liver metastasis: a 5-year single-centre experience. HPB (Oxford) 2011; 13: 745-52.

33. Mehta NN, Ravikumar R, Coldham CA, Buckels JA, Hubscher SG, Bramhall SR, et al. Effect of preoperative chemotherapy on liver resection for colorectal liver metastases. Eur J Surg Oncol 2008; 34: 782-6. 
34. Gibson PR, Fraser JR, Colman JC, Jones PA, Jennings G, Dudley FJ. Change in serum hyaluronan: a simple index of short-term drug-induced changes in hepatic sinusoidal perfusion. Gastroenterology 1993; 105: 470-4.

35. Naber AH, Van Haelst U, Yap SH. Nodular regenerative hyperplasia of the liver: an important cause of portal hypertension in non-cirrhotic patients. J Hepatol 1991; 12: 94-9.

36. Alston-Smith J, Pertoft H, Laurent TC. Hyaluronan synthesis by rat liver stellate cells is enhanced under endotoxic conditions. Matrix 1993; 13: 313-22.

37. Deleve LD, Wang $X$, Guo Y. Sinusoidal endothelial cells prevent rat stellate cell activation and promote reversion to quiescence. Hepatology 2008; 48: 920-30.

38. Rubbia-Brandt L, Audard V, Sartoretti P, Roth AD, Brezault C, Le Charpentier M, et al. Severe hepatic sinusoidal obstruction associated with oxaliplatin-based chemotherapy in patients with metastatic colorectal cancer. Ann Oncol 2004; 15: 460-6.

39. Lindqvist U, Westerberg G, Bergstrom M, Torsteindottir I, Gustafson S, Sundin A, et al. [11C]Hyaluronan uptake with positron emission tomography in liver disease. Eur J Clin Invest 2000; 30: 600-7.

40. Lindqvist U, Groth T, Loof L, Hellsing K. A hyaluronan-loading test applied to patients with liver and joint diseases. Clin Chim Acta 1992; 210: 119-32.

41. Rubbia-Brandt L, Tauzin S, Brezault C, Delucinge-Vivier C, Descombes P, Dousset B, et al. Gene expression profiling provides insights into pathways of oxaliplatin-related sinusoidal obstruction syndrome in humans. Mol Cancer Ther 2011; 10: 687-96.

42. Agostini J, Benoist S, Seman M, Julie C, Imbeaud S, Letourneur F, et al. Identification of molecular pathways involved in oxaliplatin-associated sinusoidal dilatation. J Hepatol 2012; 56: 869-76.

43. Constans J, Conri C. Circulating markers of endothelial function in cardiovascular disease. Clin Chim Acta 2006; 368: 33-47.

44. Strasberg SM. Nomenclature of hepatic anatomy and resections: a review of the Brisbane 2000 system. J Hepatobiliary Pancreat Surg 2005; 12: 351-5. 


\section{Chapter 9}

\section{Hepatic sinusoidal obstruction syndrome reduces the effect of oxaliplatin in patients with colorectal liver metastases}

Adapted from:

Vreuls CP, van den Broek MA, Winstanley A, Koek GH, Wisse E, Dejong CH, Olde Damink SW, Bosman FT, Driessen A. Hepatic sinusoidal obstruction syndrome (SOS) reduces the effect of oxaliplatin in colorectal liver metastases. 


\section{ABSTRACT}

\section{Background}

Oxaliplatin is an important chemotherapeutic agent that is used to treat patients with colorectal liver metastases (CLMs). Its administration results in reduction of tumour load which makes curative resection possible in case of initially unresectable disease. Treatment with oxaliplatin-based chemotherapy has significant side effects, as oxaliplatin can induce sinusoidal obstruction syndrome (SOS) in the non-tumour-bearing liver. SOS is associated with increased post-resectional morbidity and decreased long-term survival. We hypothesized that SOS might impede hepatic perfusion, thereby interfering with the tumour environment and attenuating the tumour response to chemotherapy.

\section{Methods}

From the prospective liver database of Maastricht University Medical Centre, 50 consecutive patients with CLMs were selected. All patients received oxaliplatin-based neoadjuvant chemotherapy followed by partial hepatectomy. The tumour regression grade (TRG) of metastases and presence of SOS in the non-tumour-bearing liver were studied histopathologically according to standard scoring systems.

\section{Results}

Thirty-two patients (64 per cent) showed histopathological signs of SOS, classified as mild in 26 per cent and moderate-severe in 38 per cent. The response to treatment, as expressed by TRG, was grade 1 [no residual cancer] in 5 (10 per cent), grade 2 [rare residual cancer cells] in 7 (14 per cent), grade 3 [fibrosis outgrowing residual cancer cells] in 14 (28 per cent), grade 4 [residual cancer cells outgrowing fibrosis] in 16 (32 per cent) and grade 5 [absence of regressive changes] in 8 patients (16 per cent), respectively. Advanced SOS grade was associated with a higher TRG $(p=0.016)$.

\section{Conclusion}

The presence of SOS goes along with a lower tumour response to oxaliplatin-based neoadjuvant chemotherapy. Sinusoidal injury may induce hepatic hypoperfusion and hypoxia, which possibly reduce the response to chemotherapy. 


\section{INTRODUCTION}

In 2008, colorectal cancer was the second most commonly diagnosed cancer in females and the third in males, responsible for 1.2 million new cancer cases worldwide ${ }^{1}$. The prognosis of patients with colorectal cancer is mainly determined by the presence of metastatic disease. The liver is the most common site of metastases and at the time of diagnosis of the colorectal primary, 15 to 20 per cent of patients have already developed colorectal liver metastases $(\mathrm{CLMs})^{2,3}$. If all CLMs can be removed by surgery, a five-year overall survival rate of around 50 per cent can be achieved ${ }^{4-6}$. Unfortunately, most patients are initially not eligible for liver surgery with curative intent due to multiple factors, such as high number of metastases, large lesion size or bilateral involvement. Currently, patients with initially unresectable disease are treated with chemotherapy, aiming at downsizing CLMs to enable resection and achieve potential cure ${ }^{7}$. Adam and colleagues reported a success rate of this strategy in 12.5 per cent of patients with a 5 -year survival rate of 33 per cent ${ }^{8}$. These results have been achieved by administration of combined chemotherapeutic regimen, consisting of fluorouracil, oxaliplatin and irinotecan ${ }^{9}$. These regimens reduce hepatic tumour load as evidenced by histopathological assessment of the tumour regression grade (TRG) ${ }^{10-12}$.

Treatment with oxaliplatin-based chemotherapy is, however, accompanied by significant side effects. Oxaliplatin induces sinusoidal obstruction syndrome (SOS) in the non-tumour-bearing liver in the majority of patients ${ }^{13-16}$. SOS was formerly known as hepatic veno-occlusive disease (VOD), a condition that has been described in patients after myeloablative hematopoietic stem cell transplantation. Clinical features of patients with advanced VOD include hyperbilirubinemia (bilirubin level above $2 \mathrm{mg} / \mathrm{dL}$ ), ascites, hepatomegaly, portal hypertension or weight gain ${ }^{17}$. These symptoms have also been described in patients with CLMs who received prolonged oxaliplatin-based chemotherapy treatment, but in the majority, SOS presents as a subclinical condition ${ }^{18}$. The presence of SOS is associated with higher post-resectional morbidity rates, prolonged hospital stay and decreased long-term survival ${ }^{15,16,19}$.

SOS is caused by the toxic effect of oxaliplatin on hepatic sinusoidal endothelial cells $(\mathrm{SECS})^{20,21}$. The ensuing swelling of SECs and loss of sinusoidal wall integrity impairs sinusoidal blood flow, resulting in congestive obstruction. Eventually, peliosis, centrilobular hepatic vein fibrotic obstruction, perisinusoidal fibrosis and nodular regenerative hyperplasia (NRH) can occur ${ }^{14}$.

The clinical symptoms as well as the histopathological findings suggest that SOS interferes with hepatic perfusion. Hypoperfusion might influence the tumour microenvironment and drug delivery to tumour cells. In line with this hypothesis, one would expect that patients developing SOS respond less well to chemotherapy in terms of tumour regression. The aim of the present study was to correlate the presence of SOS with TRG in patients with CLMs treated with oxaliplatin-based chemotherapy. 


\section{METHODS}

\section{Patients}

Patients with CLMs who underwent a first partial hepatectomy at Maastricht University Medical Centre between January 2008 and December 2009 were included in the present study. Exclusion criteria were (a) no oxaliplatin-based neoadjuvant chemotherapy treatment, (b) hepatectomy specimen not containing a lesion or containing a non-neoplastic lesion (e.g. cavernous hemangioma), (c) inadequate histopathological material available (e.g. absence of non-tumour-bearing liver on a distance of more than $2 \mathrm{~cm}$ from the tumour), and (d) laparoscopic liver surgery. In general, the treatment schedule consisted of 3 to 6 cycles of oxaliplatin-based chemotherapy combined with bevacizumab prior to surgery. The actual number of treatment cycles differed per patient and was dependent on the radiographic response measured by the 2000 response criteria in solid tumours $(\text { RECIST })^{22}$, the patient's physical condition and sustained side effects.

\section{Histopathological examination}

The partial hepatectomy specimens were sliced before fixation. After fixation in 4 per cent buffered formaldehyde, several tissue samples were taken from each tumour nodule, from the non-tumour-bearing liver (at a minimum distance of $2 \mathrm{~cm}$ from the lesion and avoiding the subcapsular region) and from the resection margins. The tissue samples were routinely embedded in paraffin and $4 \mu \mathrm{m}$ sections were cut. Histological sections were examined by two experienced HPB pathologists. In case of discrepancy, the section was discussed at a dual head microscope and consensus was reached.

\section{Assessment of sinusoidal injury}

Morphological analysis was based on haematoxylin and eosin (H\&E), reticulin and Trichrome Masson stains. Histopathological features containing vascular and parenchymal changes were scored according to the classification of Rubbia-Brandt and colleagues ${ }^{14}$. Based on these criteria, sinusoidal dilatation (grade 0 to 3), centrilobular vein and perisinusoidal fibrosis and steatosis (absent or present) were graded categorically.

\section{Assessment of tumour regression grade}

Morphological analysis of TRG was based on H\&E stains. Each liver metastasis was scored for TRG, using the approach described by Mandard and colleagues for the assessment of tumour regression after preoperative chemoradiotherapy of esophageal carcinoma, which was modified for liver metastases ${ }^{11}$. Based on these criteria, TRG was graded as follows: grade 1 showed absence of histological identifiable residual tumour and extensive fibrosis; grade 2 was characterized by the presence of rare residual tumour cells scattered through the fibrosis; grade 3 involved a substantial amount of residual tumour 
cells but fibrosis dominated; grade 4 showed residual tumour cells outgrowing fibrosis; and grade 5 was characterized by the absence of any tumour regression.

\section{Statistical analysis}

Data are given as mean or median dependent on the nature of the data. Pearson's chisquare test was used for categorical data. A multivariable logistic regression analysis was performed to correlate patient characteristics (age, sex, number of cycles chemotherapy) and the presence of SOS with TRG. For this analysis, we combined moderate (grade 2) and severe (grade 3 ) SOS lesions, resulting in 3 groups (no lesions, mild lesions and moderate-severe lesions). The TRG grade was divided in 2 groups, resulting in no or rare residual tumour cells (grade 1-2) versus a substantial amount of residual tumour cells (grade $3-5$ ). For all tests, a p-value below 0.050 was considered to indicate statistical significance. Statistical analyses were performed using SPSS version 15.0 for Windows (SPSS Inc. Chicago, IL, USA).

\section{RESULTS}

\section{Patients}

Between January 2008 and December 2009, 82 patients with CLMs underwent a first partial hepatectomy at Maastricht University Medical Centre. After excluding patients without oxaliplatin-based chemotherapy treatment $(n=22)$, hepatectomy specimen without tumour $(n=5)$, inadequate histopathological material $(n=3)$, and patients undergoing laparoscopic partial hepatectomy $(n=2), 50$ patients were available for analysis. Males and females were equally distributed with a mean age of 61 years (range 40-79). Mean number of oxaliplatin cycles was 6 (range 1-12).

\section{Histopathological examination}

Thirty-two out of 50 patients (64 per cent) showed SOS lesions of any grade. In more detail, 13 patients ( 26 per cent) had mild SOS lesions and 19 patients ( 38 per cent) had moderate or severe SOS lesions. Centrilobular vein and perisinusoidal fibrosis was present in 29 patients (58 per cent) and steatosis in 27 patients (54 per cent).

The TRG varied from grade 1 in 5 (10 per cent), grade 2 in 7 (14 per cent), grade 3 in 14 (28 per cent), grade 4 in 16 (32 per cent) and grade 5 in 8 patients ( 16 per cent), respectively. In patients with more than one metastasis, the TRG was similar for the different metastases. 


\section{Relation between SOS and TRG}

A higher grade of SOS was associated with a higher TRG $(p=0.016)$ with a regression coefficient of 1.416 (95 per cent confidence interval 0.265 - 2.567) (Table 9.1). TRG did not correlate with age, sex or number of cycles of oxaliplatin (Table 9.1). The presence of SOS was significantly correlated with hepatic fibrosis $(p=0.003)$, but not with steatosis or number of treatment cycles (data not shown).

Table 9.1 Regression coefficients from multivariable logistic regression analysis of the effect of patient characteristics and SOS grade on tumour regression grade.

\begin{tabular}{lcc}
\hline & $\begin{array}{c}\text { Regression coefficient } \\
\text { (95 per cent confidence interval) }\end{array}$ & p-value \\
\hline Age & $0.066(-0.015-0.148)$ & 0.111 \\
Sex & $-1.326(-2.976-0.329)$ & 0.116 \\
$\quad$ Male $(n=25)$ & & \\
$\quad$ Female $(n=25)$ & $1.416(0.265-2.567)$ & \\
SOS grade & & \\
$\quad \begin{array}{l}\text { ( }(n=18) \\
1(n=13)\end{array}$ & \\
2 and $3(n=19)$ & & \\
$\mathbf{N}$ oxaliplatin cycles & & \\
$<6(n=22)$ & $-1.121(-2.813-0.565)$ & \\
$\geq 6(n=28)$ & & \\
\hline
\end{tabular}

SOS, sinusoidal obstruction syndrome; $N$, number.

\section{DISCUSSION}

Oxaliplatin-based chemotherapy may affect the non-tumour-bearing liver, which leads to SOS. SOS is caused by toxic injury mainly targeting hepatic SECs, leading to a spectrum of lesions including sinusoidal dilatation, perisinusoidal fibrosis, $\mathrm{NRH}$ and peliosis ${ }^{14}$. Studies report that 22 to 74 per cent of patients receiving oxaliplatin-based neoadjuvant chemotherapy develop SOS of any grade ${ }^{13-16,23}$. Our study is consistent with recent literature and shows a prevalence of sinusoidal lesions of 64 per cent, which is at the upper bound of reported rates. The relatively high incidence in our cohort might have three explanations, that is the fact that only oxaliplatin-based chemotherapy regimen were included, extensive sampling of the non-tumour-bearing liver was performed and specific attention to sinusoidal lesions was paid, as it was our main study endpoint.

We found that the presence of SOS was significantly associated with a higher TRG, which corresponds with a lower tumour response, to oxaliplatin-based chemotherapy. Several explanations for this finding could be considered, in which hepatic hypoperfusion and oxidative stress play important roles. First, clinical symptoms and pathological features suggest that SOS impedes optimal hepatic perfusion, which may induce a state 
of hepatic hypoxia. Two recent studies reported hepatic upregulation of, among others, the expression of hypoxic factor (HIF-1a), hepatic fibrosis/hepatic stellate cell activation (COL3a1, COL3a2, PDGF-A, TIMP-1, and MMP-2), oxidative stress and angiogenic factors (VEGF-C) in oxaliplatin-related SOS relative to controls, suggesting that livers suffering from SOS are in a hypoxic state ${ }^{24,25}$. Tumour hypoxia stimulates angiogenesis and cell signalling, in pathways such as c-met and lysyl oxidase-mediated signalling, which might promote tumour cell invasion ${ }^{26}$. Our observations indirectly support these findings by the negative correlation between SOS and tumour response. It would be clinically relevant to study polymorphisms in, for example, anti-oxidant genes that may identify subgroups of patients prone to develop SOS and, concomitantly, respond poorly to chemotherapy. A second explanation involves a reduced penetration of oxaliplatin at the tumour sites secondary to the obliterative sinusoidal lesions, which might lead to a reduced cytotoxic effect of oxaliplatin.

To the best of our knowledge, this is the first study that shows a significant association between the presence of SOS and higher TRG. Previous studies showed that TRG was important for the prediction of long-term outcome in patients with CLMs treated by chemotherapy and liver surgery ${ }^{10,12}$. Rubbia-Brandt and colleagues found no significant correlation between SOS and TRG, though this was not the main focus of their paper ${ }^{10}$. As the present study was solely histopathologically based and had a short follow-up, the long-term survival data described in aforementioned papers could not be confirmed. A recent study by Tamandl and colleagues demonstrated that the presence of SOS was associated with early CLM recurrence, evidencing even more that SOS plays an important role in changing the tumour environment which may affect survival rates ${ }^{19}$.

In summary, advanced stage SOS is associated with lower tumour response to oxaliplatin-based neoadjuvant chemotherapy in patients with CLMs. This may impede the likelihood of curative surgical resection and decrease long-term survival rates. We suggest that hypoperfusion and hypoxia are important factors, but further studies are needed to elucidate the exact underlying pathophysiological mechanisms. 


\section{REFERENCES}

1. Jemal A, Bray F, Center MM, Ferlay J, Ward E, Forman D. Global cancer statistics. CA Cancer J Clin 2011; 61:69-90.

2. Adam R. Colorectal cancer with synchronous liver metastases. Br J Surg 2007; 94: 129-31.

3. Abdalla EK, Adam R, Bilchik AJ, Jaeck D, Vauthey JN, Mahvi D. Improving resectability of hepatic colorectal metastases: expert consensus statement. Ann Surg Oncol 2006; 13: 1271-80.

4. Choti MA, Sitzmann JV, Tiburi MF, Sumetchotimetha W, Rangsin R, Schulick RD, et al. Trends in long-term survival following liver resection for hepatic colorectal metastases. Ann Surg 2002; 235: 759-66.

5. Fernandez FG, Drebin JA, Linehan DC, Dehdashti F, Siegel BA, Strasberg SM. Five-year survival after resection of hepatic metastases from colorectal cancer in patients screened by positron emission tomography with F-18 fluorodeoxyglucose (FDG-PET). Ann Surg 2004; 240: 438-47; discussion 447-50.

6. Nathan H, de Jong MC, Pulitano C, Ribero D, Strub J, Mentha G, et al. Conditional survival after surgical resection of colorectal liver metastasis: an international multi-institutional analysis of 949 patients. J Am Coll Surg 2010; 210: 755-64, 764-6.

7. Wicherts DA, de Haas RJ, Adam R. Bringing unresectable liver disease to resection with curative intent. Eur J Surg Oncol 2007; 33 Suppl 2: S42-51.

8. Adam R, Delvart V, Pascal G, Valeanu A, Castaing D, Azoulay D, et al. Rescue surgery for unresectable colorectal liver metastases downstaged by chemotherapy: a model to predict long-term survival. Ann Surg 2004; 240: 644-57.

9. Alberts SR, Horvath WL, Sternfeld WC, Goldberg RM, Mahoney MR, Dakhil SR, et al. Oxaliplatin, fluorouracil, and leucovorin for patients with unresectable liver-only metastases from colorectal cancer: a North Central Cancer Treatment Group phase II study. J Clin Oncol 2005; 23: 9243-9.

10. Rubbia-Brandt L, Giostra E, Brezault C, Roth AD, Andres A, Audard V, et al. Importance of histological tumor response assessment in predicting the outcome in patients with colorectal liver metastases treated with neo-adjuvant chemotherapy followed by liver surgery. Ann Oncol 2007; 18: 299-304.

11. Mandard AM, Dalibard F, Mandard JC, Marnay J, Henry-Amar M, Petiot JF, et al. Pathologic assessment of tumor regression after preoperative chemoradiotherapy of esophageal carcinoma. Clinicopathologic correlations. Cancer 1994; 73: 2680-6.

12. Blazer DG, Kishi Y, Maru DM, Kopetz S, Chun YS, Overman MJ, et al. Pathologic response to preoperative chemotherapy: a new outcome end point after resection of hepatic colorectal metastases. J Clin Oncol 2008; 26: 5344-51.

13. Rubbia-Brandt L, Audard V, Sartoretti P, Roth AD, Brezault C, Le Charpentier M, et al. Severe hepatic sinusoidal obstruction associated with oxaliplatin-based chemotherapy in patients with metastatic colorectal cancer. Ann Oncol 2004; 15: 460-6.

14. Rubbia-Brandt L, Lauwers GY, Wang H, Majno PE, Tanabe K, Zhu AX, et al. Sinusoidal obstruction syndrome and nodular regenerative hyperplasia are frequent oxaliplatin-associated liver lesions and partially prevented by bevacizumab in patients with hepatic colorectal metastasis. Histopathology 2010; 56: 430-9.

15. Soubrane O, Brouquet A, Zalinski S, Terris B, Brezault C, Mallet V, et al. Predicting high grade lesions of sinusoidal obstruction syndrome related to oxaliplatin-based chemotherapy for colorectal liver metastases: correlation with post-hepatectomy outcome. Ann Surg 2010; 251: 454-60. 
16. Nakano H, Oussoultzoglou E, Rosso E, Casnedi S, Chenard-Neu MP, Dufour P, et al. Sinusoidal Injury Increases Morbidity After Major Hepatectomy in Patients With Colorectal Liver Metastases Receiving Preoperative Chemotherapy. Ann Surg 2008; 247: 118-124.

17. Jones RJ, Lee KS, Beschorner WE, Vogel VG, Grochow LB, Braine HG, et al. Venoocclusive disease of the liver following bone marrow transplantation. Transplantation 1987; 44: 778-83.

18. Hubert C, Sempoux C, Horsmans Y, Rahier J, Humblet Y, Machiels JP, et al. Nodular regenerative hyperplasia: a deleterious consequence of chemotherapy for colorectal liver metastases? Liver Int 2007; 27: 938-43.

19. Tamandl D, Klinger M, Eipeldauer S, Herberger B, Kaczirek K, Gruenberger B, et al. Sinusoidal obstruction syndrome impairs long-term outcome of colorectal liver metastases treated with resection after neoadjuvant chemotherapy. Ann Surg Oncol 2010; 18: 421-30.

20. DeLeve LD, Shulman HM, McDonald GB. Toxic injury to hepatic sinusoids: sinusoidal obstruction syndrome (veno-occlusive disease). Semin Liver Dis 2002; 22: 27-42.

21. DeLeve LD, McCuskey RS, Wang X, Hu L, McCuskey MK, Epstein RB, et al. Characterization of a reproducible rat model of hepatic veno-occlusive disease. Hepatology 1999; 29: 1779-91.

22. Therasse P, Arbuck SG, Eisenhauer EA, Wanders J, Kaplan RS, Rubinstein L, et al. New guidelines to evaluate the response to treatment in solid tumors. European Organization for Research and Treatment of Cancer, National Cancer Institute of the United States, National Cancer Institute of Canada. J Natl Cancer Inst 2000; 92: 205-16.

23. Overman MJ, Maru DM, Charnsangavej C, Loyer EM, Wang H, Pathak P, et al. Oxaliplatin-mediated increase in spleen size as a biomarker for the development of hepatic sinusoidal injury. J Clin Oncol 2010; 28: 2549-55.

24. Rubbia-Brandt L, Tauzin S, Brezault C, Delucinge-Vivier C, Descombes P, Dousset B, et al. Gene expression profiling provides insights into pathways of oxaliplatin-related sinusoidal obstruction syndrome in humans. Mol Cancer Ther 2011; 10: 687-96.

25. Agostini J, Benoist S, Seman M, Julie C, Imbeaud S, Letourneur F, et al. Identification of molecular pathways involved in oxaliplatin-associated sinusoidal dilatation. J Hepatol 2012; 56: 869-76.

26. Allen M, Louise Jones J. Jekyll and Hyde: the role of the microenvironment on the progression of cancer. J Pathol 2010; 223: 162-76. 



\section{Chapter 10}

\section{The flavonoid monoHER prevents monocrotaline- induced hepatic sinusoidal injury in rats}

Adapted from:

Ezzat T, van den Broek MA, Davies N, Dejong CH, Bast A, Malagó M, Dhar DK, Olde Damink SW. The flavonoid monoHER prevents monocrotaline-induced hepatic sinusoidal injury in rats. J Surg Oncol 2012; 106: 72-8. 


\section{ABSTRACT}

\section{Background}

Sinusoidal obstruction syndrome (SOS) develops in 50 to 70 per cent of patients after oxaliplatin-based treatment for colorectal liver metastases. SOS is associated with portal hypertension and is caused by oxidative damage to endothelial cells and subsequent matrix metalloproteinase (MMP) induction. We studied the effect of the flavonoid monoHER on SOS prevention.

\section{Methods}

A monocrotaline (MCT) model was used to induce SOS in rats, with pre-treatment with monoHER. Portal pressure, hepatocellular damage, hepatic inflammation and MMP expression were studied in vivo. The potential inhibition of oxaliplatin-associated cytotoxicity by monoHER was tested in vitro in colorectal cancer cell lines.

\section{Results}

MonoHER ameliorated the increase in portal pressure after MCT ( $72 \mathrm{hr}: 7.3 \pm 2.7$ versus $11.4 \pm 3.0 \mathrm{mmHg}, \mathrm{p}=0.016, \mathrm{MCT}+$ monoHER versus MCT). MonoHER also prevented hepatocellular damage reflected by ALAT ( $72 \mathrm{hr}$ : $129.5 \pm 114.5$ versus $311.9 \pm 163.6 \mathrm{IU} / \mathrm{L}$, $\mathrm{p}=0.028, \mathrm{MCT}+$ monoHER versus $\mathrm{MCT}$ ). The histological liver damage score was lower in the monoHER group ( $72 \mathrm{hr}: 4.8 \pm 3.6$ versus $10.3 \pm 0.5, \mathrm{p}=0.002, \mathrm{MCT}+$ monoHER versus $\mathrm{MCT}$ ) concomitant with less inflammatory cell infiltration. Livers of MCT treated rats had higher expression of MMP-9 when compared to their MCT+monoHER counterparts at both $24 \mathrm{hr}(\mathrm{p}=0.016)$ and $72 \mathrm{hr}(\mathrm{p}<0.001)$. MonoHER had no effect on the in vitro proliferation of colorectal cancer cells when used either alone or in combination with oxaliplatin.

\section{Conclusion}

MonoHER prevented MCT-induced portal hypertension and hepatic injury in an experimental rat model. In vitro experiments showed no effect of monoHER on the cytotoxicity of oxaliplatin in colorectal cancer cells. 


\section{INTRODUCTION}

Partial liver resection remains the only single modality to achieve curative treatment for colorectal liver metastases (CLMs), but unfortunately only 15 to 25 per cent of patients are initially eligible for resection ${ }^{1}$. The introduction of systemic chemotherapy regimens including irinotecan and oxaliplatin improved resectability rates ${ }^{1,2}$. Oxaliplatin-based chemotherapy is related however, to the development of hepatic sinusoidal obstruction syndrome $(\mathrm{SOS})^{3}$. Different SOS grades resulting from oxaliplatin develop in about 50 to 70 per cent of patients. SOS is associated with increased post-resectional morbidity secondary to portal hypertension and intra-operative hemorrhage, which results in a prolonged hospital stay ${ }^{4-8}$. Rare, fatal cases of SOS have been reported following oxaliplatin-based neoadjuvant chemotherapy ${ }^{9,10}$.

The pathophysiological mechanism of hepatic SOS has been well studied in the monocrotaline (MCT) rat model ${ }^{11}$. In short, monocrotaline or other toxic agents, such as oxaliplatin, cause damage to sinusoidal endothelial cells (SECs) by depletion of glutathione, which leads to depolymerization of F-actin. This, in turn, causes swelling of SECs and increased synthesis and activity of matrix metalloproteinases (MMPs), which break down the extracellular matrix. As a result, there is loss of SEC fenestration and formation of gaps between SECs allowing erythrocytes to penetrate into the space of Disse. The blood flow then dissects and separates the SEC lining, which subsequently blocks the sinusoids. Secondary to these events, an inflammatory reaction, characterized by accumulation of neutrophils in the sinusoidal region, occurs ${ }^{12,13}$.

Interventions to prevent the development of SOS focus on agents that either deactivate MMP activity, restore glutathione levels or preserve sinusoidal perfusion ${ }^{14-16}$. These agents have solely been tested in experimental models with mixed, but promising results and validation in clinical trials is pending. 7-Monohydroxyethylrutoside (monoHER) is a potential protective agent against SOS because of its favorable effect on the microvascular endothelium and its antioxidant and anti-inflammatory capacities ${ }^{17}$. In the past decade, our group has shown that monoHER provides a protective effect against doxorubicin-induced cardiotoxicity in mice without interfering with its anti-tumour activity ${ }^{18,19}$ and has tested it in phase I and II clinical trials in humans without any serious side effects ${ }^{20,21}$.

The aim of this study was to assess the protective effect of the flavonoid monoHER on the development of MCT-induced sinusoidal injury in vivo in rats. Furthermore, the effect of monoHER on cell growth of human colorectal cancer cells exposed to increasing concentrations of oxaliplatin was determined in vitro. 


\section{METHODS}

\section{Animals}

This study was approved by the Animal Research Committee of University College London. All animal experiments were conducted according to Home Office guidelines under the UK Animals and Scientific Procedures Act 1986. Male Sprague-Dawley rats (Charles-Rivers, UK, $n=63$ ), weighing 230-280 grams, were given free access to standard rodent chow and water, serially weighed, with a light/dark cycle of $12 \mathrm{hr}$, at a temperature of $22-23^{\circ} \mathrm{C}$ and 50 per cent relative humidity.

\section{Experimental design}

The study consisted of 2 separate experiments, an in vivo study that investigated the effect of monoHER on portal pressure and hepatocellular damage in rats, and an in vitro experiment that studied the effect of monoHER + oxaliplatin on cell growth in human colorectal cancer cells. A rat model for SOS using a single gavage of MCT (MCT, Sigma AIdrich, St. Louis, MO, USA) was used for the in vivo study ${ }^{11}$. MonoHER was kindly provided by the Department of Pharmacology and Toxicology (Maastricht University Medical Centre, Maastricht, the Netherlands).

\section{Study 1}

Animals were divided into three groups; (Group 1) MCT+monoHER group ( $n=21)$ received a single gavage of $160 \mathrm{mg} / \mathrm{kg} \mathrm{MCT} \mathrm{in} 0.5 \mathrm{ml}$ dimethyl sulfoxide (DMSO) and 500 $\mathrm{mg} / \mathrm{kg}$ monoHER dissolved in $36 \mathrm{mM} \mathrm{NaOH}$ (pH 7.8-8) intraperitoneally (i.p.) starting 1 day before MCT treatment and continued once daily up until sacrifice; (Group 2) MCT group ( $n=21$ ) received a single gavage of $160 \mathrm{mg} / \mathrm{kg} \mathrm{MCT} \mathrm{in} 0.5 \mathrm{ml}$ DMSO and the vehicle of monoHER (0.5 $\mathrm{ml}$ of $36 \mathrm{mM} \mathrm{NaOH})$ i.p. on the same time points as Group 1; and (Group 3) Sham group $(n=21)$, received a single gavage of the vehicle of MCT ( 0.5 $\mathrm{ml} \mathrm{DMSO})$ on day 1 and the vehicle of monoHER $(0.5 \mathrm{ml}$ of $36 \mathrm{mM} \mathrm{NaOH})$ i.p. at the same time points as Group 1. Rats were studied at $24 \mathrm{hr}, 48 \mathrm{hr}$ and $72 \mathrm{hr}$ after MCT gavage $(n=$ 7 at each time point).

Study 2

In order to examine the effect of MCT and monoHER on the cytotoxic effect of oxaliplatin in human colorectal cancer cells (LoVo and LS174T), increasing concentrations of oxaliplatin were added to these cells in combination with MCT and monoHER in vitro. 


\section{Study endpoints}

\section{Study 1}

The primary endpoint was difference in portal pressure (PP) between the intervention and control group. Secondary endpoints were amount of liver damage assessed by alanine aminotransferase (ALAT) levels and histological examination, extent of sinusoidal injury assessed by electron microscopy, amount of hepatic inflammation, and MMP expression.

\section{Study 2}

The primary endpoint was cell viability measured by MTS-assay. The MTS-assay is a colorimetric assay that analyzes the activity of enzymes that reduce MTS to formazan, which allows assessing the viability and proliferation of cells in cell cultures.

\section{Measurement of portal pressure}

Rats were anaesthetized with inhalation of isoflurane. The body temperature was maintained at $37^{\circ} \mathrm{C}$. A midline laparotomy was performed and the portal vein was cannulated with PE-5 tubing (VWR International, Leicestershire, UK) without disturbing the surrounding structures. Portal pressure (PP) was continuously monitored and recorded using a pressure transducer, a pressure amplifier and a computer equipped with a data recording and analysis system (BIOPAC systems, Norfolk, UK). The average pressure over a $5 \mathrm{~min}$ period was considered as the final portal pressure. Hereafter, blood samples were taken from the abdominal aorta and liver tissue was cut from the median lobe at sacrifice. A part of the liver tissue was preserved immediately in liquid nitrogen and the remaining portion in 10 per cent neutral formalin for downstream applications. Blood samples were drawn in pre-chilled tubes for EDTA-plasma, heparin-plasma and serum collection. Samples were stored at $-80^{\circ} \mathrm{C}$ until determination.

\section{Measurement of liver damage}

Serum ALAT was measured using COBAS integra 400 biochemistry analyzer (Roche, Indianapolis, IN, USA). Paraffin sections were stained with haematoxylin and eosin (H\&E) using standard histological techniques. Histopathological examination was performed in a blinded fashion by an experienced HPB pathologist. Liver damage was quantified using a modification of the scoring system described by Rubbia-Brandt and colleagues ${ }^{4}$, including sinusoidal dilatation (graded on a 0 to 3 scale), perisinusoidal hemorrhage, peliosis, hepatocellular necrosis (graded on a 0 to 3 scale) and inflammatory cell infiltration (graded on a 0 to 3 scale). Mononuclear inflammatory cells were counted in three most densely populated areas at high power fields (HPF) (x400) and the average number of cells was considered as the number of cells/HPF. 


\section{Evaluation of MMP-2 and MMP-9 expression in tissue}

Formalin-fixed tissue sections ( $5 \mu \mathrm{m}$ thick) were used for immunohistochemistry. The immunohistochemical study was performed using the streptavidin ABC duet kit (Dako, Cambridgeshire, UK). Briefly, sections were dewaxed in xylene, rehydrated in graded alcohol and rinsed with PBS. Antigen retrieval was done by heating the sections in citrate buffer solution at $95^{\circ} \mathrm{C}$ for $10 \mathrm{~min}$. Endogenous peroxidase activity was blocked by 3 per cent hydrogen peroxide for $20 \mathrm{~min}$. The sections were incubated with the following primary antibodies: monoclonal rabbit anti-MMP-9 (1:200) (Abcam, Cambridge, UK) and monoclonal mouse anti-MMP-2 (1:200), (Abcam, Cambridge, UK). Streptavidin-peroxidase labelled secondary antibody was applied for $30 \mathrm{~min}$ at room temperature. Colour was developed with the chromagen 3,3'-diaminobenzidine (Dako, Cambridgeshire, UK) and counterstained with Mayer's haematoxylin solution. A scoring system based on both the intensity (graded on a 0 to 3 scale) and extent of staining ( $1=<33$ per cent, $2=$ 33-67 per cent, $3=>67$ per cent) was used as a semi-quantitative measurement of MMP expression ${ }^{22}$.

\section{Evaluation of ultrastructural damage by electron microscopy}

Perfusion fixation of the total liver in situ was performed according to the protocol that was detailed recently by our group ${ }^{23}$.

\section{In vitro effect of monoHER on human colorectal cancer cell growth}

The effect of treatment with monoHER, MCT and oxaliplatin on colorectal cancer cell proliferation was assessed using the MTS-assay (Promega, Southampton, UK). Briefly, 2000 cells/well in a 96 well plate were incubated in RPMI-1640 media (Invitrogen, Paisley, UK) with increasing concentrations of (1) oxaliplatin 50-100-200 $\mu \mathrm{M}$, (2) MCT 2-4-8 mM and (3) monoHER 25-50-100 mM or a combination of two of those. Following incubation for $48 \mathrm{hr}, 20 \mathrm{ul}$ of MTS reagent was added in each well and incubated for another $2 \mathrm{hr}$ at $37^{\circ} \mathrm{C}$ in a $\mathrm{CO}_{2}$ incubator. The absorbance was read at $490 \mathrm{~nm}$ using an LT-4000MS spectrophotometer (Labtech International, East Sussex, UK).

\section{Statistical analysis}

Data are given as mean with standard deviation. ANOVA with post-hoc test was used for multigroup analyses. This was performed using SPSS version 15.0 for Windows (SPSS Inc. Chicago, IL, USA). A p-value below 0.050 was considered statistically significant. 


\section{RESULTS}

\section{MonoHER reduced the portal pressure in SOS}

MCT administration increased the PP compared to sham animals (Figure 10.1A). Pretreatment with monoHER attenuated the increase in PP at all three time points (24hr: $7.4 \pm 0.9$ versus $9.4 \pm 1.0 \mathrm{mmHg}, p=0.005,48 \mathrm{hr}: 7.9 \pm 1.3$ versus $10.2 \pm 1.3 \mathrm{mmHg}, p=0.019$, and $72 \mathrm{hr}$ : $7.3 \pm 2.7$ versus $11.4 \pm 3.0 \mathrm{mmHg}, \mathrm{p}=0.016$, all $\mathrm{MCT}+$ monoHER versus MCT, respectively). There was no difference in PP between $\mathrm{MCT}+$ monoHER and sham animals (24hr: $7.4 \pm 0.9$ versus $6.5 \pm 0.7 \mathrm{mmHg}, \mathrm{p}=0.227,48 \mathrm{hr}$ : $7.9 \pm 1.3$ versus $7.5 \pm 1.1 \mathrm{mmHg}$, $\mathrm{p}=0.783$, and $72 \mathrm{hr}: 7.3 \pm 2.7$ versus $6.6 \pm 0.5 \mathrm{mmHg}, \mathrm{p}=0.860$, all $\mathrm{MCT}+\mathrm{monoHER}$ versus sham, respectively).

A

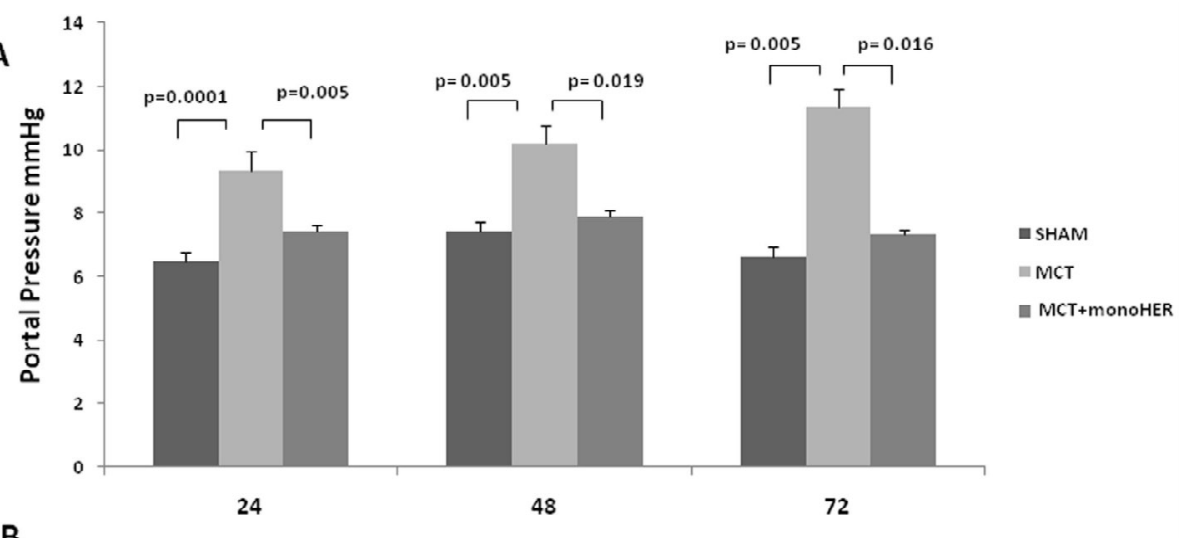

B

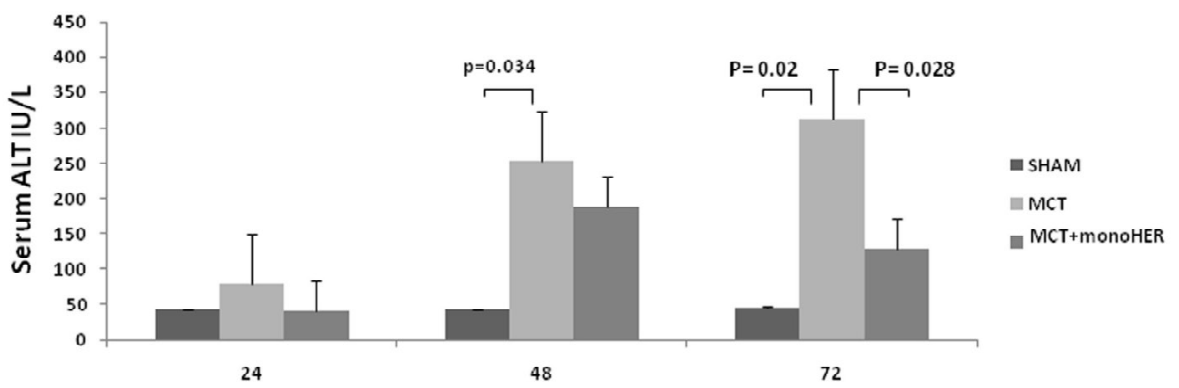

Figure 10.1A and 10.1B PP and serum ALAT measurements in the studied groups.

(A) MCT administration caused a significant rise in PP in the MCT group compared to the sham group. Pretreatment with monoHER significantly reduced PP at all time points compared to the MCT group. (B) Rats pre-treated with monoHER had significantly reduced serum ALAT at $72 \mathrm{hr}$ compared to the MCT group. There was no difference between both groups at $24 \mathrm{hr}$ or $48 \mathrm{hr}$. MCT rats had higher serum ALAT levels compared to sham rats at $48 \mathrm{hr}$ and $72 \mathrm{hr}$, but not at $24 \mathrm{hr}$.

Legend: MCT, monocrotaline; PP portal pressure; ALAT, alanine aminotransferase. 


\section{Liver damage was attenuated by monoHER}

All animals survived the experiment. At sacrifice, the livers in the MCT treated rats appeared macroscopically congested at $24 \mathrm{hr}$ and had patchy necrotic areas at $48 \mathrm{hr}$ and $72 \mathrm{hr}$. The macroscopic appearance of livers in the MCT+monoHER group was less congested at 48hr and $72 \mathrm{hr}$ (Figure 10.2).

Serum ALAT levels were higher in the MCT treated rats compared to sham rats at 48hr and $72 \mathrm{hr}$ (48hr: $253.4 \pm 171.7$ versus $42.2 \pm 3.1 \mathrm{IU} / \mathrm{L}, \mathrm{p}=0.034$, and $72 \mathrm{hr}$ : $311.9 \pm 163.6$ versus $46.2 \pm 4.3 \mathrm{IU} / \mathrm{L}, \mathrm{p}=0.002, \mathrm{MCT}$ versus sham, respectively). MCT+monoHER rats had lower ALAT concentrations when compared to MCT rats at $72 \mathrm{hr}(129.5 \pm 114.5$ versus 311.9 $\pm 163.6 \mathrm{IU} / \mathrm{L}, \mathrm{p}=0.028$ ). There was no difference in serum ALAT between MCT+monoHER and MCT rats at $24 \mathrm{hr}(p=0.092)$ and $48 \mathrm{hr}(p=1.000)$ (Figure 10.1B).

MCT administration resulted in extensive sinusoidal dilatation on histological examination, whereas a much lesser extent of dilatation was noticed in the MCT+monoHER group at $24 \mathrm{hr}$ (Figure 10.3A and 10.3B). At 48hr, marked congestion developed in the MCT as well as the MCT+monoHER groups with a much more extensive infiltration of inflammatory cells in the MCT compared to the MCT+monoHER group (Figure $10.3 \mathrm{C}$ and 10.3D). Bridging hepatic necrosis across the hepatic lobules along with

$24 \mathrm{H}$
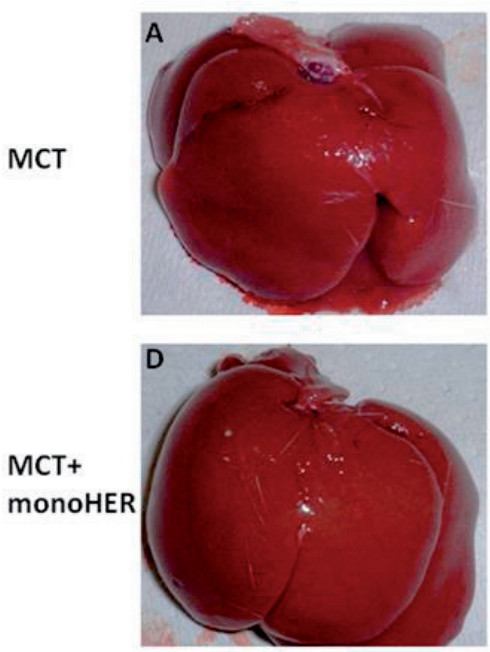

$48 \mathrm{H}$
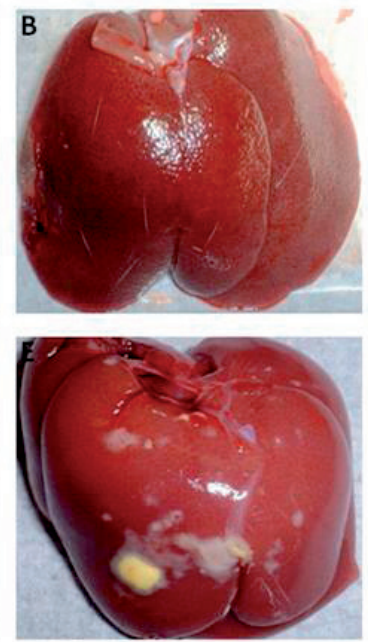

$72 \mathrm{H}$
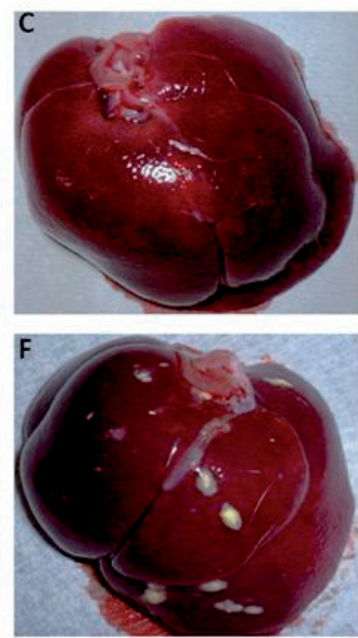

Figure 10.2A-F Representative samples showing the macroscopic appearance of the liver at different time points.

(A-C) The congestion is evident in the MCT group at $24 \mathrm{hr}$, with appearance of punctuate hemorrhagic spots at $48 \mathrm{hr}$ and areas of frank necrosis in a deeply congested liver at $72 \mathrm{hr}$, which resembles the blue liver seen in the clinical scenario. (D-F) Congestion also occurred in the MCT+monoHER group, however much less severe than in the MCT group and without development of punctuate hemorrhage or necrosis. Of important notice, the yellow color on the liver represents monoHER and not necrosis.

Legend: MCT, monocrotaline. 

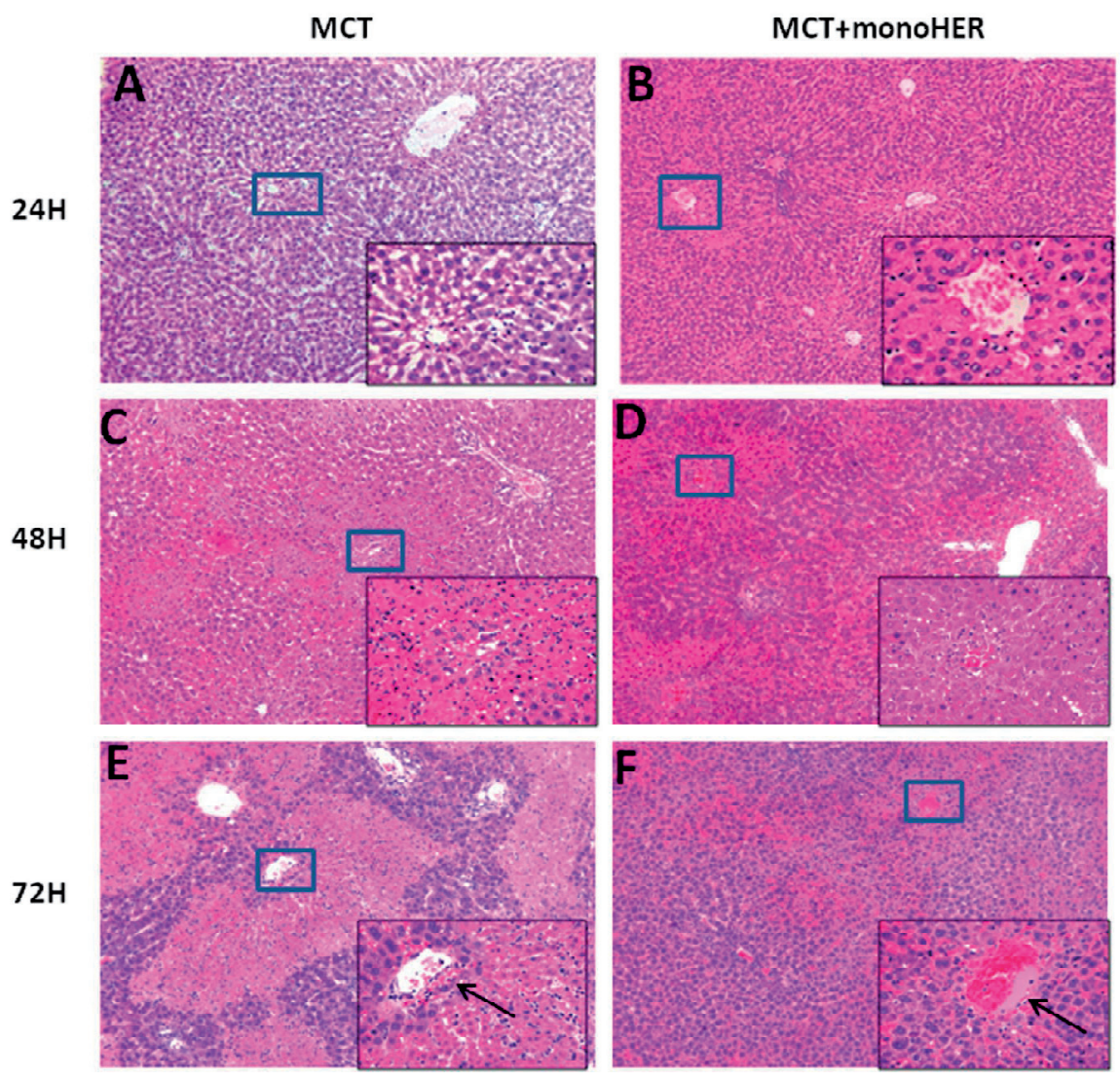

Figure 10.3A-F Representative photomicrographs of liver damage on histological level in the studied groups.

(A-B) There was more severe sinusoidal dilatation in the MCT group at $24 \mathrm{hr}$ compared to the MCT+monoHER group. (C-D) At 48hr, the sinusoidal dilatation was associated with hepatocellular damage mainly in the centrilobular zones in both groups, however with less inflammatory cells in the MCT+monoHER group. (EF) At $72 \mathrm{hr}$, there was massive necrosis all over the liver in the MCT group with marked inflammation with disruption of the SEC lining and extravasation of erythrocytes and inflammatory cells in direct contact with the hepatocytes. Necrosis did not develop in livers of MCT+monoHER rats and the integrity of sinusoidal cell lining was maintained in this group.

Legend: MCT, monocrotaline; SEC, sinusoidal endothelial cells; original magnification $\times 40$, inset $\times 200$.

dense inflammatory cell infiltration was observed in the MCT group at $72 \mathrm{hr}$. Also, SEC detachment from the underlying basement membrane with evidence of subendothelial hemorrhage was observed in the MCT group (Figure 10.3E). In contrast, in the $\mathrm{MCT}+$ monoHER group, congestion was still present; however, hepatocellular necrosis and endothelial cell detachment were minimal (Figure 10.3F). The overall liver damage severity score was lower in the MCT+monoHER group at $72 \mathrm{hr}(4.8 \pm 3.6$ versus $10.3 \pm 0.5$, $\mathrm{MCT}+$ monoHER versus MCT, $\mathrm{p}=0.002$ ) (Figure 10.4A). Inflammatory cell infiltration/HPF 

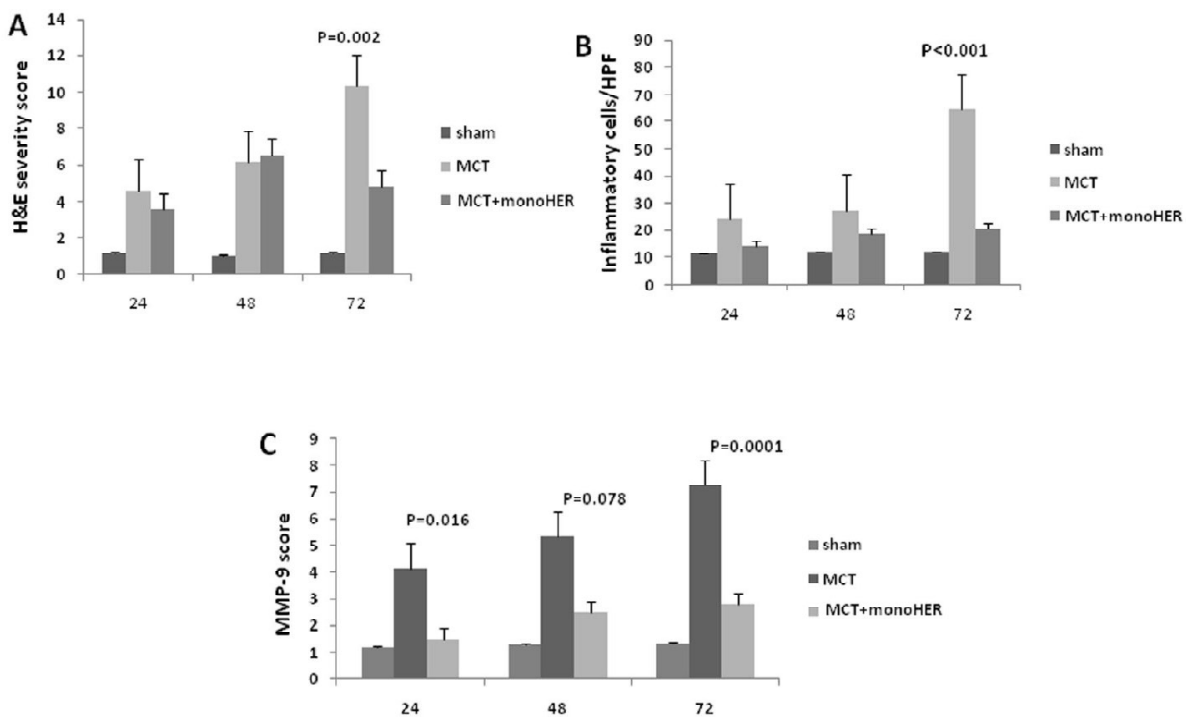

Figure 10.4A-C Semi-quantification of liver damage and MMP activity.

(A-B) The overall liver damage severity score and inflammatory cell count/HPF was significantly lower in the $\mathrm{MCT}+$ monoHER group at $72 \mathrm{hr}$ only compared to the MCT group. (C) MMP-9 expression was significantly lower in the MCT+monoHER group at $24 \mathrm{hr}$ and $72 \mathrm{hr}$ compared to the MCT group.

Legend: HPF, high power field; MCT, monocrotaline; MMP, matrix metalloproteinase.

was also lower in the MCT+monoHER group at $72 \mathrm{hr}(20.6 \pm 8.3$ versus $64.5 \pm 9.0$ cells/ $\mathrm{HPF}, \mathrm{MCT}+$ monoHER versus $\mathrm{MCT}, \mathrm{p}<0.001$ ), but not at any other time point (Figure 10.4B).

\section{Ultrastructural damage was attenuated after monoHER}

On scanning electron microscopy (SEM), large gaps were noticed in the sinusoids of the MCT group with less gap formation in the MCT+monoHER group. On transmission electron microscopy (TEM), gapping of the fenestrae was evident in the MCT group with detachment of the SEC lining from the basement membrane and disruption of the space of Disse. There was a slight degree of SEC separation that could be detected in the MCT+monoHER group without disruption of the space of Disse (Figure 10.5).

\section{MonoHER treatment reduced MMP-9 expression in the liver}

Livers of MCT treated rats had higher expression of MMP-9 when compared to MCT+monoHER rats at both $24 \mathrm{hr}(\mathrm{p}=0.016)$ and $72 \mathrm{hr}(\mathrm{p}<0.001)$ (Figure 10.4C). Areas of highest expression included SEC lining and to lesser extent inflammatory cells in the MCT group with less expression in the MCT+monoHER group (Figure 10.6A-D). In addition, MMP-9 expression was noticed in areas surrounding the hepatocellular necrosis at $72 \mathrm{hr}$ which was not present in the MCT+monoHER group (Figure 10.6E and 10.6F). In 
MCT
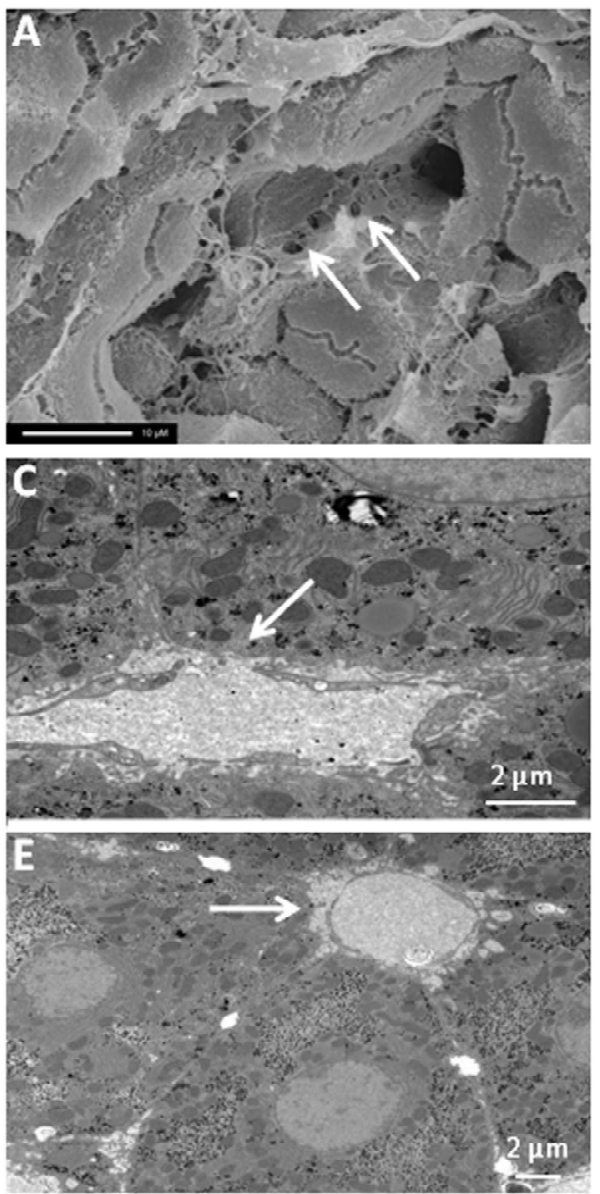

MCT+monoHER
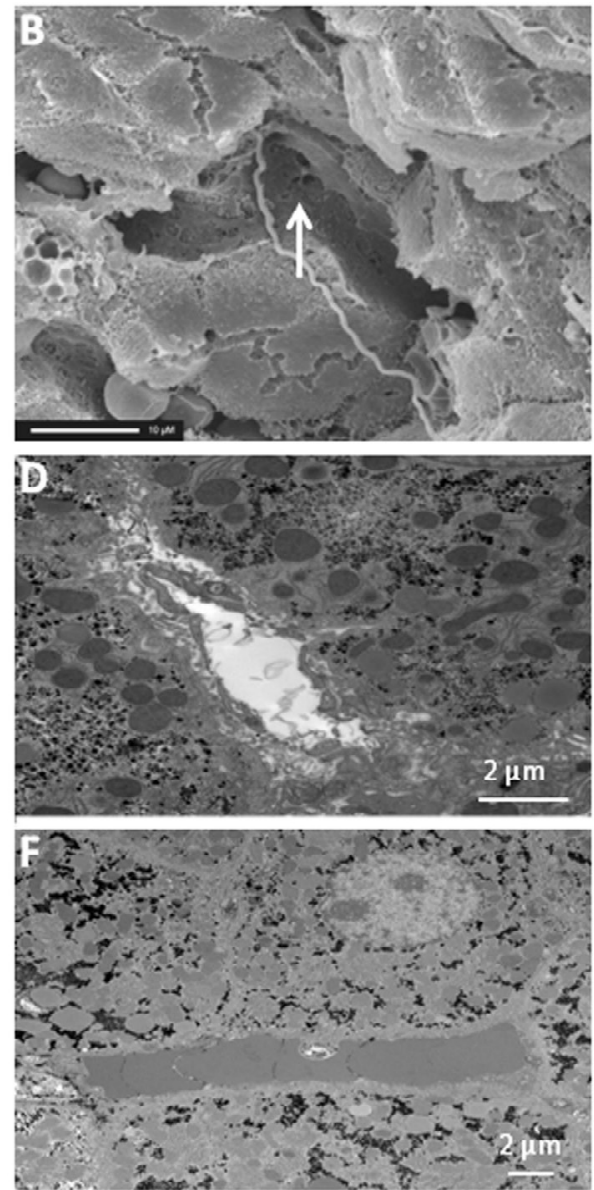

Figure 10.5A-F Electron microscopy of the liver at $72 \mathrm{hr}$.

(A-B) Treatment with MCT induced damage at the structural level in the form of gap formation in the liver sinusoids with damage still present in the monoHER group, however with fewer gaps as seen on SEM. (C) TEM studies showed that there was gapping of the sinusoids in the MCT group leading to an increase in the size of fenestrae from the normal size of about $150 \mathrm{~nm}$ to about $200 \mathrm{~nm}$. (E) Detachment of the SEC lining from the basement membrane was detected in the MCT group. (D-F) In the MCT+monoHER group, the integrity of the SEC lining was maintained with slight separation from the basement membrane but no disruption of the space of Disse.

Legend: SEM, scanning electron microscopy; TEM, transmission electron microscopy; SEC, sinusoidal endothelial cell; MCT, monocrotaline.

contrast to MMP-9 expression, MMP-2 expression was very weak in liver sections and no obvious differences could be detected among the three groups. 
MCT
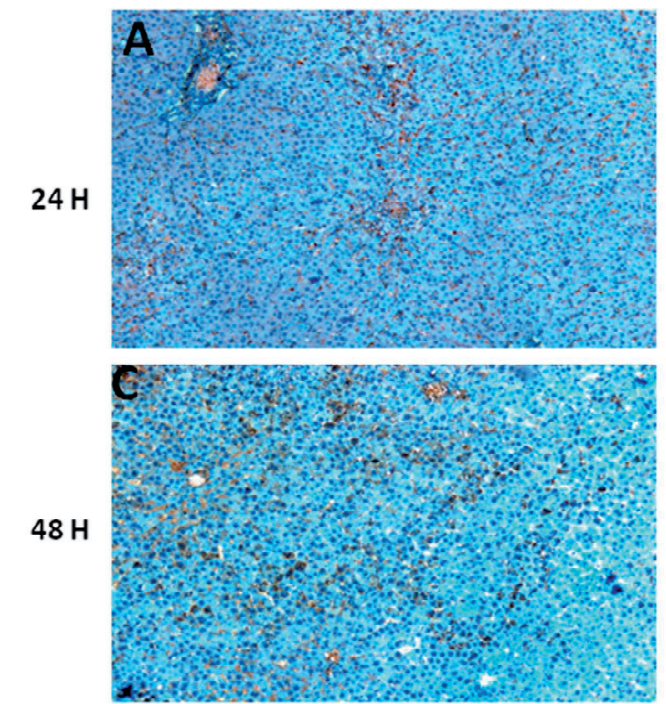

$48 \mathrm{H}$

$72 \mathrm{H}$

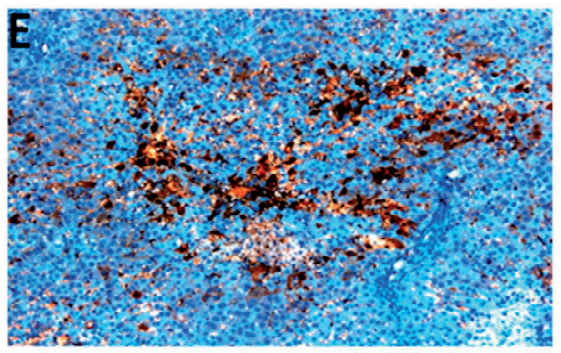

MCT+monoHER
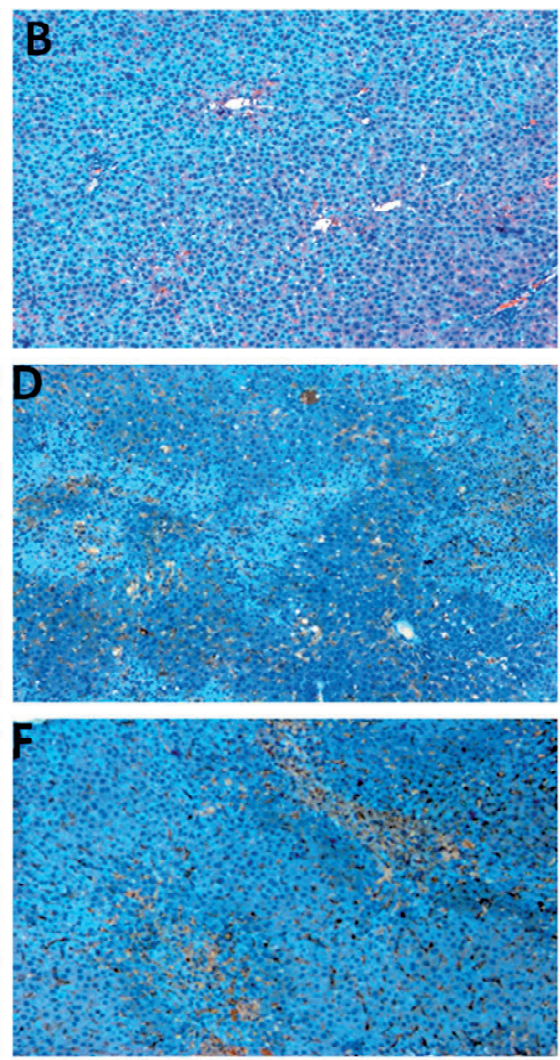

Figure 10.6A-F MMP-9 expression in the liver.

(A-B) MMP-9 was expressed as early as after 24hr in MCT group in the SEC lining, mainly in the centrilobular regions with less expression in the MCT+monoHER group. (C-F) An increase in expression was evident at $48 \mathrm{hr}$ and $72 \mathrm{hr}$ in the MCT group with less expression in the MCT+monoHER group.

Legend: MMP, matrix metalloproteinase; $M C T$, monocrotaline; original magnification $\times 40$.

\section{MonoHER did not interfere with oxaliplatin cytotoxicity}

Both colorectal cancer cell lines showed dose-dependent cytotoxicity for increasing doses of oxaliplatin. MCT and/or monoHER at different concentrations did not have any significant effect on cell numbers in both cell lines. Addition of monoHER to oxaliplatin, even in high dose $(100 \mu \mathrm{M})$, did not interfere with the cytotoxic effect of oxaliplatin (Figure 10.7A and 10.7B). 


\section{A. Lovo cells}

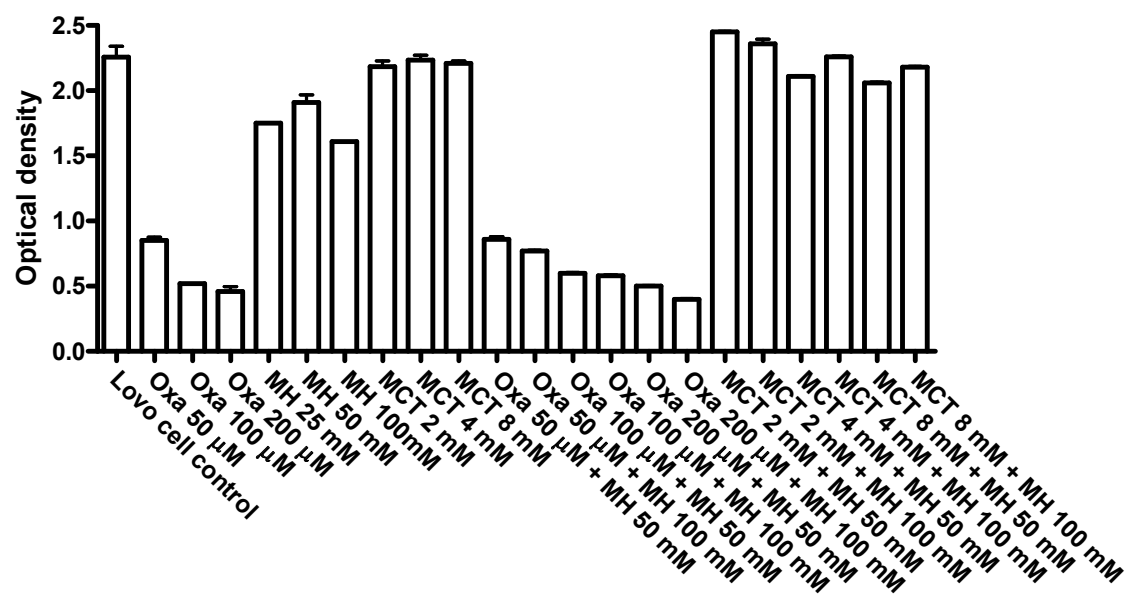

B. LS174T cells

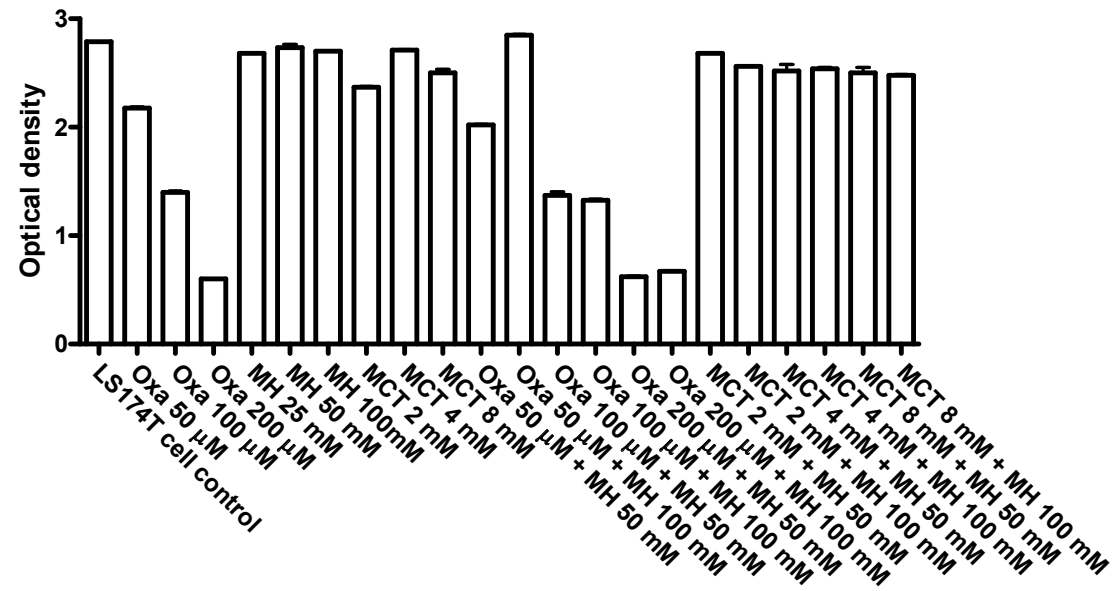

Figure 10.7A and 10.7B In vitro analysis of proliferation of colorectal cancer cell lines.

(A-B) MonoHER did not interfere with the dose-dependent cytotoxic effects of oxaliplatin. MCT and monoHER alone had no effect on viable cell count in either cell line (A: Lovo cells and B: LS174T cells).

Legend: oxa, oxaliplatin; $\mathrm{MH}$, monoHER; MCT, monocrotaline.

\section{DISCUSSION}

The present study clearly demonstrated that monoHER can be used to prevent the development of portal hypertension and hepatocellular damage in an experimental model of SOS. Initially, we tried to establish a rat model of oxaliplatin-induced SOS, but this attempt was not successful. In a series of preliminary experiments, the maximum tolerable dose of oxaliplatin in rats (10 and $20 \mathrm{mg} / \mathrm{kg}$ i.p., respectively) was used. Oxali- 
platin treatment induced only mild congestion without any significant hepatocellular necrosis, which is one of the main pathognomonic features of $\mathrm{SOS}^{11}$. The actual reason is unknown and might be related to an altered metabolism of oxaliplatin in particular species such as rats. These findings compelled us to use the reproducible MCT model for induction of $\mathrm{SOS}^{11}$.

We found that the PP in the MCT+monoHER group was significantly lower compared to the MCT group at all time points. This was also reflected in the gross macroscopic appearance of the liver in the MCT+monoHER group, which demonstrated less severe liver congestion compared to the MCT group, specifically at $72 \mathrm{hr}$. Similarly, monoHER prevented hepatocellular damage reflected by lower aminotransferase levels, less histological liver damage and less inflammatory cell infiltration. These observations could be explained by the pharmacological properties of monoHER, which can act as (1) endothelial cell stabilizer, (2) potent anti-oxidant and (3) anti-inflammatory compound. These capacities make monoHER a potential drug for the prevention of SOS as endothelial damage, oxidative stress and inflammation all are involved in its pathogenesis ${ }^{24}$.

First, swelling of SECs followed by their detachment is key in the development of SOS and portal hypertension ${ }^{13}$. MonoHER is one of the constituents of Venoruton ${ }^{\circledR}, \mathrm{a}$ drug that has been shown to protect the microvascular endothelium, as evidenced by a reduced number of circulating endothelial cells in patients with chronic venous insufficiency ${ }^{25,26}$. The subendothelial localization of monoHER is thought to be partially responsible for these positive protective effects on the endothelium ${ }^{27}$.

Secondly, it is well known that both MCT and oxaliplatin induce SOS by formation of reactive oxygen species secondary to glutathione depletion in $\mathrm{SECs}{ }^{11,28}$. Flavonoids such as monoHER have been shown to possess potent free radical scavenging properties and possibly, these anti-oxidant capacities played a role in prevention of SOS by monoHER through sweeping free oxygen radicals ${ }^{29}$. Earlier observations by DeLeve and colleagues showed, however, that SOS could be prevented by intraportal glutathione infusion, but not by infusion of other potent free radical scavengers ${ }^{16}$.

Third, a recent study on the pathogenesis of SOS showed that acute phase pathway genes were upregulated in liver tissue following oxaliplatin therapy ${ }^{30}$. In the present study, monoHER played a pivotal role in reducing cell damage and inflammation secondary to MCT treatment, as evidenced by less structural liver damage, significantly lower serum aminotransferase levels and reduced number of inflammatory cells in the MCT+monoHER group. The reduced number of inflammatory cells could either be attributable to less damage and therefore a reduced stimulus for an inflammatory response or direct prevention of adhesion of neutrophils to sinusoidal endothelial cells in the liver. Indeed, we showed previously that monoHER attenuated doxorubicin-induced inflammatory reactions by inhibition of neutrophil adhesion to endothelial cells through down-regulation of VCAM and E-selectin ${ }^{31}$. Narita and colleagues also demonstrated 
that inhibition of neutrophil adhesion to the sinusoids is the key factor in prevention of SOS following MCT intake in rats ${ }^{12}$. On the contrary, Hanumegowda and colleagues undermined the role of polymorphonuclear cells (PMNs) in MCT-induced SOS by showing PMN infiltration predominantly away from the centrilobular areas, which represent the most heavily affected areas of sinusoidal damage ${ }^{32}$. In addition, PMN depletion did not prevent the sinusoidal damage. Although PMNs might not apparently play a significant role in sinusoidal damage, they definitely play an integral role in hepatocellular damage in several ways including release of superoxide radicals and activated MMP- $9^{32}$.

The present study also showed a role of monoHER in interfering with another important downstream pathway in SOS development, which is MMP-9 expression. The main sources of MMP-9 expression are PMNs and SECs ${ }^{32-34}$. The MCT pyrrole, which is the active metabolite of MCT, interacts with F-actin in SECs leading to F-actin depolymerization and swelling of SECs. F-actin depolymerization is linked to increased MMP activity in the extracellular matrix. The upregulation of MMPs leads to degradation of basement membrane components and subsequent SEC detachment ${ }^{13}$. DeLeve and colleagues noticed high expression of MMP-9 and to a lesser extent MMP-2 in the basement membrane of SECs after MCT and concluded that this might be responsible for separation and dissection of the SEC from the basement membrane ${ }^{15}$. In the present study, MMP-9 expression was restricted predominantly in the SEC lining in the first 24 to $48 \mathrm{hr}$, however, at $72 \mathrm{hr}$, expression extended to areas of hepatocellular damage with significantly less expression in the MCT+monoHER group. It can be hypothesized that monoHER plays a pivotal role in the stabilization of the basement membrane by diminished MMP-9 expression due to maintenance of SEC integrity or diminished inflammatory cell influx.

The advantage of monoHER lies within its effectiveness in preventing SOS without interference with the cytotoxic activity of oxaliplatin. MonoHER itself had no effect on the growth rate of colorectal cancer cells and, when used along the cytotoxic doses of oxaliplatin, it did not interfere with the anti-proliferative effects of oxaliplatin. This is in agreement with our previous results, where monoHER achieved adequate intracellular concentration without interfering with the pharmacokinetics of doxorubicin ${ }^{18,35}$. In addition, monoHER has been proven to be safe in a phase I clinical trial and has been tested in a phase II clinical trial in man ${ }^{20,21}$. Recently, concerns have been raised towards the oxidation product of monoHER, which may be toxic ${ }^{29}$. Similar to quercetin, the oxidation product of monoHER reacts in vitro and in vivo with the thiol group of glutathione, possibly leading to its depletion. This would lead to catastrophic consequences following monoHER therapy since the primary insult in SOS is glutathione depletion. Fortunately, this has not been shown to occur in patients owing to a structural difference between monoHER and quercetin. Unlike quercetin, which easily reacts with protein thiols, oxidized monoHER is reduced by its preferential binding with plasma ascorbate, hence the risk of glutathione depletion is minimal ${ }^{36}$. 
To conclude, monoHER is a potential therapeutic agent to be used in prevention of oxaliplatin-induced SOS owing to its potent vasoprotective, anti-oxidant and antiinflammatory effects ameliorating portal hypertension, sinusoidal injury and hepatocellular damage in an experimental SOS rat model. 


\section{REFERENCES}

1. Abdalla EK, Vauthey JN. Chemotherapy prior to hepatic resection for colorectal liver metastases: helpful until harmful? Dig Surg 2008; 25: 421-9.

2. Abdalla EK, Adam R, Bilchik AJ, Jaeck D, Vauthey JN, Mahvi D. Improving resectability of hepatic colorectal metastases: expert consensus statement. Ann Surg Oncol 2006; 13: 1271-80.

3. Rubbia-Brandt L, Audard V, Sartoretti P, Roth AD, Brezault C, Le Charpentier M, et al. Severe hepatic sinusoidal obstruction associated with oxaliplatin-based chemotherapy in patients with metastatic colorectal cancer. Ann Oncol 2004; 15: 460-6.

4. Rubbia-Brandt L, Lauwers GY, Wang H, Majno PE, Tanabe K, Zhu AX, et al. Sinusoidal obstruction syndrome and nodular regenerative hyperplasia are frequent oxaliplatin-associated liver lesions and partially prevented by bevacizumab in patients with hepatic colorectal metastasis. Histopathology 2010; 56: 430-9.

5. Narita M, Oussoultzoglou E, Chenard MP, Rosso E, Casnedi S, Pessaux P, et al. Sinusoidal obstruction syndrome compromises liver regeneration in patients undergoing two-stage hepatectomy with portal vein embolization. Surg Today 2011; 41: 7-17.

6. Slade JH, Alattar ML, Fogelman DR, Overman MJ, Agarwal A, Maru DM, et al. Portal hypertension associated with oxaliplatin administration: clinical manifestations of hepatic sinusoidal injury. Clin Colorectal Cancer 2009; 8: 225-30.

7. Dixon E, Datta I, Sutherland FR, Vauthey JN. Blood loss in surgical oncology: neglected quality indicator? J Surg Oncol 2009; 99: 508-12.

8. Soubrane O, Brouquet A, Zalinski S, Terris B, Brezault C, Mallet V, et al. Predicting high grade lesions of sinusoidal obstruction syndrome related to oxaliplatin-based chemotherapy for colorectal liver metastases: correlation with post-hepatectomy outcome. Ann Surg 2010; 251: 454-60.

9. van den Broek MA, Olde Damink SW, Driessen A, Dejong CH, Bemelmans $\mathrm{MH}$. Nodular regenerative hyperplasia secondary to neoadjuvant chemotherapy for colorectal liver metastases. Case Report Med 2009; 2009: 457975.

10. Bilchik AJ, Poston G, Curley SA, Strasberg S, Saltz L, Adam R, et al. Neoadjuvant chemotherapy for metastatic colon cancer: a cautionary note. J Clin Oncol 2005; 23: 9073-8.

11. DeLeve LD, McCuskey RS, Wang X, Hu L, McCuskey MK, Epstein RB, et al. Characterization of a reproducible rat model of hepatic veno-occlusive disease. Hepatology 1999; 29: 1779-91.

12. Narita M, Hatano E, Tamaki N, Yamanaka K, Yanagida A, Nagata H, et al. Dai-kenchu-to attenuates rat sinusoidal obstruction syndrome by inhibiting the accumulation of neutrophils in the liver. $J$ Gastroenterol Hepatol 2009; 24: 1051-7.

13. DeLeve LD, Ito Y, Bethea NW, McCuskey MK, Wang X, McCuskey RS. Embolization by sinusoidal lining cells obstructs the microcirculation in rat sinusoidal obstruction syndrome. Am J Physiol Gastrointest Liver Physiol 2003; 284: G1045-52.

14. DeLeve LD, Wang X, Kanel GC, Ito Y, Bethea NW, McCuskey MK, et al. Decreased hepatic nitric oxide production contributes to the development of rat sinusoidal obstruction syndrome. Hepatology 2003; 38: 900-8.

15. Deleve LD, Wang X, Tsai J, Kanel G, Strasberg S, Tokes ZA. Sinusoidal obstruction syndrome (venoocclusive disease) in the rat is prevented by matrix metalloproteinase inhibition. Gastroenterology 2003; 125: 882-90.

16. Wang X, Kanel GC, DeLeve LD. Support of sinusoidal endothelial cell glutathione prevents hepatic veno-occlusive disease in the rat. Hepatology 2000; 31: 428-34. 
17. Bast A, Haenen GR, Bruynzeel AM, Van der Vijgh WJ. Protection by flavonoids against anthracycline cardiotoxicity: from chemistry to clinical trials. Cardiovasc Toxicol 2007; 7: 154-9.

18. van Acker SA, Boven E, Kuiper K, van den Berg DJ, Grimbergen JA, Kramer K, et al. Monohydroxyethylrutoside, a dose-dependent cardioprotective agent, does not affect the antitumor activity of doxorubicin. Clin Cancer Res 1997; 3: 1747-54.

19. van Acker SA, Kramer K, Grimbergen JA, van den Berg DJ, van der Vijgh WJ, Bast A. Monohydroxyethylrutoside as protector against chronic doxorubicin-induced cardiotoxicity. Br J Pharmacol 1995; 115: 1260-4.

20. Willems AM, Bruynzeel AM, Kedde MA, van Groeningen CJ, Bast A, van der Vijgh WJ. A phase I study of monohydroxyethylrutoside in healthy volunteers. Cancer Chemother Pharmacol 2006; 57: 678-84.

21. Bruynzeel AM, Niessen HW, Bronzwaer JG, van der Hoeven JJ, Berkhof J, Bast A, et al. The effect of monohydroxyethylrutoside on doxorubicin-induced cardiotoxicity in patients treated for metastatic cancer in a phase II study. Br J Cancer 2007; 97: 1084-9.

22. Dhar DK, Wang TC, Tabara H, Tonomoto Y, Maruyama R, Tachibana M, et al. Expression of trefoil factor family members correlates with patient prognosis and neoangiogenesis. Clin Cancer Res 2005; 11:6472-8.

23. Wisse E, Braet F, Duimel H, Vreuls C, Koek G, Olde Damink SW, et al. Fixation methods for electron microscopy of human and other liver. World J Gastroenterol 2010; 16: 2851-66.

24. DeLeve LD. Hepatic microvasculature in liver injury. Semin Liver Dis 2007; 27: 390-400.

25. Wadworth AN, Faulds D. Hydroxyethylrutosides. A review of its pharmacology, and therapeutic efficacy in venous insufficiency and related disorders. Drugs 1992; 44: 1013-32.

26. Cesarone MR, Belcaro G, Pellegrini L, Ledda A, Vinciguerra G, Ricci A, et al. Circulating endothelial cells in venous blood as a marker of endothelial damage in chronic venous insufficiency: improvement with venoruton. J Cardiovasc Pharmacol Ther 2006; 11: 93-8.

27. Neumann HA, Carlsson K, Brom GH. Uptake and localisation of O-(beta-hydroxyethyl)-rutosides in the venous wall, measured by laser scanning microscopy. Eur J Clin Pharmacol 1992; 43: 423-6.

28. Rabik CA, Dolan ME. Molecular mechanisms of resistance and toxicity associated with platinating agents. Cancer Treat Rev 2007; 33: 9-23.

29. Jacobs H, van der Vijgh WJ, Koek GH, Draaisma GJ, Moalin M, van Strijdonck GP, et al. Characterization of the glutathione conjugate of the semisynthetic flavonoid monoHER. Free Radic Biol Med 2009; 46: 1567-73.

30. Rubbia-Brandt L, Tauzin S, Brezault C, Delucinge-Vivier C, Descombes P, Dousset B, et al. Gene expression profiling provides insights into pathways of oxaliplatin-related sinusoidal obstruction syndrome in humans. Mol Cancer Ther 2011; 10: 687-96.

31. Abou El Hassan MA, Verheul HM, Jorna AS, Schalkwijk C, van Bezu J, van der Vijgh WJ, et al. The new cardioprotector Monohydroxyethylrutoside protects against doxorubicin-induced inflammatory effects in vitro. Br J Cancer 2003; 89: 357-62.

32. Hanumegowda UM, Copple BL, Shibuya M, Malle E, Ganey PE, Roth RA. Basement membrane and matrix metalloproteinases in monocrotaline-induced liver injury. Toxicol Sci 2003; 76: 237-46.

33. Borregaard N, Cowland JB. Granules of the human neutrophilic polymorphonuclear leukocyte. Blood 1997; 89: 3503-21.

34. Upadhya AG, Harvey RP, Howard TK, Lowell JA, Shenoy S, Strasberg SM. Evidence of a role for matrix metalloproteinases in cold preservation injury of the liver in humans and in the rat. Hepatology 1997; 26: 922-8. 
35. Abou El Hassan MA, Kedde MA, Zwiers UT, Bast A, van der Vijgh WJ. The cardioprotector monoHER does not interfere with the pharmacokinetics or the metabolism of the cardiotoxic agent doxorubicin in mice. Cancer Chemother Pharmacol 2003; 51: 306-10.

36. Jacobs H, Moalin M, Bast A, van der Vijgh WJ, Haenen GR. An essential difference between the flavonoids monoHER and quercetin in their interplay with the endogenous antioxidant network. PLoS One 2010; 5: e13880. 



\section{Part IV}

Summary and future perspectives 

Chapter 11

Summary and general discussion 

There has been substantial progress in the field of liver surgery owing to improved patient selection, novel surgical techniques and optimization of perioperative care ${ }^{1}$. As a result, morbidity and mortality rates after partial liver resection have gradually declined $^{2,3}$. Due to the enhanced safety, the indications for partial hepatectomy have broadened towards more extensive resections in high-risk patient groups. The resultant small remnant liver volume and impaired preoperative liver function pose a risk of postresectional liver failure (PLF). Although the incidence of PLF is relatively low, it may have deleterious consequences and is associated with high mortality rates (chapter 1). Risk factors for PLF are either surgery or patient-related. As curative treatment options are lacking to date, prevention of PLF is of vital importance to improve outcomes of liver surgery.

The broader aim of this thesis was to assess and improve outcomes of patients undergoing liver surgery. This then translated into three more specific aims looking at (1) the feasibility of randomized controlled trials (RCTs) in liver surgery using clinical endpoints, (2) surgery-related risk factors of PLF and (3) patient-related risk factors of PLF. Taken together, the aims of the present thesis were as follows:

Aim 1: to develop strategies that increase the feasibility of conducting RCTs in liver surgery using clinical outcomes as primary endpoints (chapter 2, 3 and 4).

Aim 2: to evaluate the effect of two surgical techniques on hepatic damage in patients undergoing liver surgery (chapter 5 and 6).

Aim 3: to study the effect of oxaliplatin-based chemotherapy on hepatic damage in patients undergoing partial liver resection for colorectal liver metastases (CLMs) (chapter 7, 8, 9 and 10).

\section{PART I. ASSESSING OUTCOMES OF LIVER SURGERY: CRITICAL APPRAISAL OF CLINICAL ENDPOINTS}

The first aim of this thesis was to develop strategies that increase the feasibility of performing RCTs in liver surgery using clinical endpoints.

In the current era of evidence-based surgical practice, treatment recommendations are preferentially based on high level of evidence research, such as RCTs or meta-analyses. The performance of RCTs in the field of liver surgery is challenging, which may explain the low number of randomized studies performed in this area to date ${ }^{4,5}$. The challenges are mainly related to practical issues, such as difficulty of recruiting a sufficient number of patients in a timely manner, and design issues, such as the selection of a suitable primary endpoint that accurately reflects the effect of the intervention ${ }^{6}$. Traditionally, short-term clinical endpoints such as operative mortality and total morbidity are used for the evaluation of surgical interventions ${ }^{7}$. Therefore, part I of this thesis focuses on 
facilitating the conduct of RCTs using clinical outcomes of liver surgery as primary endpoints.

In chapter 2, the feasibility of conducting an RCT in liver surgery using a single component, clinical outcome as primary endpoint was evaluated. A systematic literature review was performed to estimate the mean incidence rate of surgery-related mortality and specific types of morbidity in current hepatobiliary (HPB) practice. Mean operative mortality rate was 1.0 per cent and mean total morbidity rate 28.9 per cent; mean rates of bile leakage and post-resectional liver failure were 4.4 and 2.6 per cent, respectively. Based on these mean incidence rates, the smallest numbers of patients needed in each arm of an RCT using a clinical outcome as primary endpoint were calculated. As a rule of thumb, the sample size of a trial is negatively correlated to the incidence rate of the outcome parameter under study ${ }^{8}$. Consequently, the computed sample sizes turned out to be extremely large. An RCT aiming to show a one-third relative reduction in operative mortality had to include 15614 patients in each arm. The same RCT aiming to show a one-third relative reduction in PLF had to include 5924 patients in each arm. As approximately 500 liver resections are performed annually in the Netherlands and approximately 7000 in the United States, we concluded that the feasibility of conducting an adequately powered liver surgery-related RTC using a single component, clinical outcome as primary endpoint was low. We postulated that strategies facilitating the conduct of high level of evidence research in liver surgery using clinical outcomes as primary endpoints include the use of composite endpoints (CEPs), organization of multicentre trials and/or performance of meta-analyses. Alternatively, liver surgery-related surrogate endpoints can be adopted as primary endpoints, but robust validation of the latter is lacking 9 .

In order to facilitate the use of CEPs and conduct of meta-analyses, uniform definitions of complications of liver surgery are needed ${ }^{10}$. The use of standard definitions reduces inconsistencies in trial reporting, allows for unequivocal interpretation of trial data and facilitates comparison of trial results ${ }^{7}$. In chapter $\mathbf{3}$, standard definitions of clinical outcomes of liver surgery were proposed. Results of a systematic literature review showed that clear definitions of clinical endpoints of RCTs in liver surgery were only provided in less than one-third of trial reports, and, if present, differed substantially. A web-based survey among 54 international experts in HPB surgery was undertaken in order to reach consensus on definitions of the most frequently used clinical endpoints of RCTs in liver surgery. The survey had a response rate of 57 per cent. Based on the comments of the respondents, the proposed definitions were adjusted. These final definitions are open to discussion and need to be validated in large, prospective patient cohorts.

In advance of formal validation, the proposed definitions were used for the design of a liver surgery specific CEP in chapter 4. A CEP is a combination of two or more procedure specific outcomes that are considered as a single, dichotomous endpoint ${ }^{11}$. 
CEPs lead to an increased statistical power because their event rate is higher than that of a single component, clinical endpoint. As a consequence, the sample size needed for an adequately powered RCT decreases likewise. Based on an electronic survey among international experts in HPB surgery, a consensus-based and well-defined liver surgery specific CEP was developed which included ascites, PLF, bile leakage, intra-abdominal hemorrhage, intra-abdominal abscess, and operative mortality, all occurring within 90 days of initial liver surgery. Caution should be exercised when interpreting CEPs, as they can be misleading if components vary widely in clinical relevance or incidence ${ }^{12,13}$. Therefore, all components of the liver surgery specific CEP had to be classified as ClavienDindo severity grade of at least $3^{14}$. In addition, the incidence rate of the liver surgery specific CEP and its components was evaluated in 815 patients who had undergone liver surgery in two high-volume European HPB centres. The incidence of the individual components ranged between 1 and 10 percent. The incidence of the liver surgery specific CEP was 11 per cent in one cohort and 19 per cent in the other. These rates led to an approximately twofold reduction in the sample size required for an adequately powered RCT using the liver surgery specific CEP instead of a single component, clinical endpoint as primary endpoint. The clinical utility of the proposed liver surgery specific CEP seems promising, however, prospective evaluation is warranted. Moreover, it may be necessary to design CEPs customized for medication or surgical technique trials, to assign weight to its components or to develop CEPs that include long-term outcomes of liver surgery ${ }^{15}$.

In conclusion, the results of part I of this thesis showed that the feasibility of conducting adequately powered RCTs in liver surgery using surgery-related mortality or morbidity as primary endpoint was low, as such endpoints require large sample sizes owing to their low incidence. The implementation of a consensus-based and well-defined liver surgery specific CEP consisting of ascites, PLF, bile leakage, intra-abdominal hemorrhage, intra-abdominal abscess, and operative mortality was able to overcome this problem. Prospective validation of the latter CEP is warranted.

\section{PART II. ASSESSING OUTCOMES OF LIVER SURGERY: HEPATIC DAMAGE AS A RESULT OF SURGICAL TECHNIQUE}

The second aim of the present thesis was to evaluate the effect of different surgical techniques on hepatocellular damage in patients undergoing liver surgery.

Intra-operative blood loss and the need for blood transfusions are related to adverse short- and long-term outcomes of liver surgery ${ }^{16-18}$. Procedures to limit blood loss include mobilization of the liver and intermittent occlusion of the hepatoduodenal ligament (i.e. Pringle maneuver) ${ }^{19}$. The rationale for their use is under discussion as both procedures induce profound hepatocellular damage ${ }^{20-22}$. In order to examine this liver cell damage 
in more detail, these procedures were separately assessed in two studies in humans described in part II of the present thesis.

During mobilization, the liver is forcefully manipulated in order to dissect its ligaments and control direct venous branches to the inferior caval vein. Our group previously showed that manipulation of the liver was the leading cause of surgery-induced liver cell damage ${ }^{23}$. In addition, we observed that manipulation of the liver during liver surgery was followed by a systemic inflammatory response. Animal models suggest that activation of hepatic immune cells plays a central role in the development of mobilization-induced liver injury and subsequent clinical outcome, but these results need confirmation in $\operatorname{man}^{24}$. It is important to unravel the link between mobilization-induced liver cell damage and hepatic inflammation, as it is possible to modulate this inflammatory response. In chapter 5, the association between liver mobilization, hepatocellular damage and hepatic inflammation was established in humans. We demonstrated that liver mobilization was associated with hepatocellular damage and hepatic inflammation, as shown by hepatic infiltration of MPO and CD68-positive inflammatory cells and hepatic upregulation of mRNA of pro-inflammatory cytokines, such as interleukin (IL) $1 \beta$, IL-6 and IL-8. In addition, there was a significant correlation between the duration of mobilization and the increase in MPO-positive cells after mobilization. The relation between manipulation-induced liver injury and post-resectional outcomes could not be established due to the relatively small patient numbers in the present study. The question therefore still remains whether the observed hepatic inflammatory response is beneficial or not. Based on aforementioned experimental data, we hypothesize that prevention of mobilization-induced liver cell damage and inflammation could be of clinical importance. Preventive strategies include either employment of alternative surgical techniques, such as the anterior approach or laparoscopic surgery, or modulation of the inflammatory response ${ }^{21,25-27}$. In order to identify subgroups that would benefit most from these preventive measures, studying differences in mobilization-induced hepatocellular damage between livers without and with underlying disease, such as chemotherapy-associated hepatotoxicity or steatohepatitis, would be relevant.

Transection of liver parenchyma usually follows mobilization of the liver. In order to limit blood loss during transection, an intermittent Pringle maneuver (IPM) may be applied. The optimal duration of the ischemic intervals during IPM is unknown and depends on the balance between ischemia-induced hepatocellular damage and blood loss, both resulting in postoperative morbidity ${ }^{28}$. Two recent RCTs showed that IPM using 30 min ischemic intervals resulted in similar remnant liver function and hepatocellular damage compared to IPM using 15 min ischemic intervals, while intra-operative blood loss was lower in the $30 \mathrm{~min}$ ischemic interval groups $\mathrm{s}^{29}, 30$. However, up until now, consequences of different ischemic intervals were assessed using aminotransferase levels on different postoperative days. It remains uncertain if the assay of aminotransferases is sufficiently 
sensitive to detect small differences in hepatocellular injury ${ }^{31}$. Intra-operative monitoring of liver fatty acid-binding protein (L-FABP) plasma levels may be a more direct and sensitive method to analyze liver cell damage. L-FABP is a cytosolic protein with a small molecular mass, which is involved in intracellular fatty acid transport and lipoprotein metabolism ${ }^{32,33}$. After an insult to the liver, a rapid rise in plasma L-FABP is observed as result of a quick release of intracellular L-FABP from damaged, dying hepatocytes ${ }^{34}$. In chapter 6, we showed in a randomized study in patients undergoing liver surgery that IPM using $30 \mathrm{~min}$ ischemic intervals resulted in similar hepatocellular damage, as reflected by L-FABP, compared to IPM using $15 \mathrm{~min}$ ischemic intervals. Moreover, remnant liver function was preserved after prolonged IPM. By measuring L-FABP fluxes over the gut, we did not find evidence for the induction of intestinal damage secondary to inflow occlusion and portovenous stasis. A follow-up study of our group, however, showed that IPM was associated with intestinal epithelial damage, reflected by increased intestinal fatty acid-binding protein levels, and gut barrier dysfunction, evidenced by endotoxemia $^{35}$. Of important notice, no IPM led to significantly less hepatocellular damage in the present RCT. As a recent meta-analysis failed to show a clinical advantage of IPM, we recommend not to use intermittent pedicle clamping during liver surgery ${ }^{20}$. If IPM is used, 30 min ischemic intervals are preferred for safe liver surgery.

In conclusion, we demonstrated in part II of the present thesis that mobilization of the liver was associated with liver cell damage and hepatic inflammation proportional to its duration. Modulation of this inflammatory response might be beneficial, although data in man are lacking. IPM using 15 or 30 minutes ischemic intervals induced comparable hepatocellular damage, reflected by systemic L-FABP levels, whereas no IPM resulted in significantly less liver cell injury. Therefore, IPM should only be used when required for safe liver surgery.

\section{PART III. ASSESSING OUTCOMES OF LIVER SURGERY: HEPATIC DAMAGE AS A RESULT OF OXALIPLATIN-BASED CHEMOTHERAPY}

The third aim of this thesis was to study hepatic sinusoidal injury secondary to oxaliplatin-based chemotherapy in patients undergoing partial liver resection for CLMs.

Liver surgery is the only treatment with the potential of long-term cure for patients with $\mathrm{CLMs}^{36}$. Unfortunately, only a minority of patients with CLMs present with initially resectable disease. Therefore, a multidisciplinary approach consisting of neoadjuvant chemotherapy followed by surgery has become standard of care. Perioperative chemotherapy treatment has proven to be able to downsize tumour mass, enhance resectability rates, and increase progression-free survival after liver surgery for $\mathrm{CLMs}^{37,38}$. However, oxaliplatin-based chemotherapy is associated with injury to hepatic sinusoidal 
endothelial cells (SECs) of the non-tumour-bearing liver, which may manifest as sinusoidal obstruction syndrome $(\mathrm{SOS})^{39-41}$. The presence of sinusoidal lesions is related to a decreased regenerative capacity and enhanced post-resectional morbidity after major liver resection, such as PLF and PLF-related death ${ }^{41-43}$. As the presence of SOS is a risk factor of PLF, we explored the clinical consequences of sinusoidal injury, analyzed a noninvasive marker for its detection and assessed a potential protective agent.

Chapter 7 illustrated the clinical problem of severe sinusoidal injury of the nontumour-bearing liver in two patients who had undergone major liver surgery for CLMs. Both patients had no history of pre-existent liver disease and were treated with 6 cycles of oxaliplatin-based chemotherapy prior to liver surgery. Histopathological analysis of the resection specimen showed severe sinusoidal injury of the liver consistent with sinusoidal congestion and nodular regenerative hyperplasia (NRH). Along with the presence of $\mathrm{NRH}$, portal hypertension developed postoperatively which led to life-threatening complications and death of one patient. Although the present report describes only two cases, the presence of sinusoidal lesions after oxaliplatin-based chemotherapy for CLMs is a frequent clinical problem with lesions of any grade reported in 22 to 74 per cent and $\mathrm{NRH}$ in 25 per cent of patients ${ }^{40,41,44-46}$. Given the toxic effects of oxaliplatin-based chemotherapy on the non-tumour-bearing liver, it seems advisable to limit its administration prior to surgery. If oxaliplatin-based neoadjuvant chemotherapy is necessary to achieve liver surgery with curative intent, preoperative detection of SOS and institution of preventive measures are important.

Information on the presence of sinusoidal injury prior to major liver surgery is clinically relevant, as it may alter the surgical strategy. As patients with SOS have a decreased functional reserve, they may benefit from restrictive surgery or two-stage procedures. The gold standard for the detection of SOS is measurement of the wedged hepatic venous pressure gradient and assessment of liver pathology ${ }^{47}$. The drawbacks of these invasive procedures are well known, and therefore, non-invasive detection methods have been developed. These methods use indirect markers of sinusoidal injury and have therefore modest sensitivity and specificity for the detection of $\mathrm{SOS}^{41,45}$. In chapter 8 , the hypothesis that systemic hyaluronic acid $(\mathrm{HA})$ level would be an accurate marker of SOS secondary to oxaliplatin-based chemotherapy in patients with CLMs was explored. $\mathrm{HA}$ is synthesized throughout the body by cells of mesodermal lineage. After the release of HA into the systemic circulation, it is rapidly cleared by a receptor-facilitated removal mechanism almost solely present in hepatic SECs ${ }^{48,49}$. Therefore, HA is regarded as a marker of hepatic SEC function. As SEC damage is a prime event in the development of SOS, we hypothesized that HA levels would differ between patients with and without SOS. In our cohort, SOS lesions of any grade were present in 58 per cent of patients treated with oxaliplatin-based chemotherapy. Systemic HA levels were significantly higher in patients with moderate or severe SOS compared to patients with no or mild 
SOS, with a fair diagnostic performance for detection of advanced SOS. The optimal cutoff value of systemic HA levels of $44.1 \mathrm{ng} / \mathrm{mL}$ yielded a sensitivity of 67 per cent and specificity of 83 per cent for detection of advanced SOS. The related negative predictive value was 91 per cent. In a subgroup of patients, the fractional extraction (FE) of HA by the liver could be calculated. The FE by the liver represents the percentage of HA that is actually taken up from the blood by the liver in relation to its inflow. Although there seemed to be a trend towards net hepatic uptake of HA in patients with no or mild SOS versus net hepatic release in patients with moderate or severe SOS, the FE of HA was not significantly different between these groups. Whether this was due to the large variation in fractional extraction of HA by the liver or actual preserved SEC function remains to be determined in a future study. Based on our results, we conclude that the increased systemic HA levels in patients with SOS did not result from decreased hepatic clearance or increased hepatic production, and may be explained by portosystemic shunting due portal hypertension or enhanced production at other sites than the liver. High HA levels identify patients at risk of SOS in whom additional investigations to detect the presence of SOS are indicted. Whether these patients benefit from restrictive liver surgery or two-stage hepatectomy combined with portal vein occlusion needs to be determined in future studies.

A recent publication showed that the presence of sinusoidal injury after oxaliplatinbased chemotherapy for CLMs was related to decreased recurrence-free and overall survival ${ }^{50}$. In patients with CLMs, disease-free and overall survival are correlated with the histological tumour response, reflected by the tumour regression grade (TRG) ${ }^{51}$. The TRG is a read out of the presence of residual tumour cells and fibrosis ${ }^{51,52}$. We hypothesized that the decreased disease-free survival in patients with SOS would be related to a decreased tumour response in these patients. Indeed, in chapter $\mathbf{9}$, we demonstrated that the tumour response was significantly decreased in patients with SOS. We postulated that a mechanism contributing to the decreased tumour response in patients with SOS was hypoperfusion due to the obliterative lesions inducing oxidative stress, which is known to induce tumour cell invasion ${ }^{53}$. Moreover, the vascular damage may have led to decreased penetration of oxaliplatin to the tumour sites. Unfortunately, long-term survival data of the patients in our cohort were lacking, as they were operated in 2008 and 2009 only, and therefore, we could not confirm a relation between SOS, TRG and long-term survival to date.

Oxidative stress and endothelial injury play an important role in the development of SOS and may therefore be potential therapeutic targets ${ }^{39}$. In chapter 10, the protective effect of monoHER on the development of SOS was studied in an experimental rat model ${ }^{54}$. MonoHER is a flavonoid with favorable effects on the microvascular endothelium and anti-oxidative and anti-inflammatory properties, making it a promising drug for the prevention of $\mathrm{SOS}^{55,56}$. MonoHER has proven to be effective in preventing doxorubicin- 
induced cardiotoxicity, which is believed to be caused by the formation of oxygen free radicals $^{55}$. In a validated rat model of SOS, pre-treatment with monoHER was able to prevent the development of portal hypertension, hepatocellular injury and endothelial cell damage after $72 \mathrm{hr}$. Mechanisms underlying the protective effect of monoHER were related to interference with SEC injury, matrix metalloproteinase expression and influx of inflammatory cells. Of important notice, there was no effect of monoHER on the cytotoxic capacity of oxaliplatin in vitro in different colorectal cancer cell lines. Further studies are needed to clarify the effect of monoHER on the preservation of remnant liver function after 70 per cent partial hepatectomy in the experimental rat model. Moreover, assessment of the efficacy of monoHER on the prevention of oxaliplatin-induced SOS in man is warranted.

In summary, we illustrated in part III of this thesis that sinusoidal injury secondary to oxaliplatin for CLMs may lead to adverse short-term clinical outcomes. However, not only short-term, but also long-term outcomes may be affected, as the tumour response was significantly decreased in patients with moderate or severe SOS. Next, we showed that systemic HA levels had a fair diagnostic performance for the detection of SOS after oxaliplatin-based chemotherapy. Patients with high HA levels should undergo additional investigations into the presence of SOS. Finally, we demonstrated that monoHER was able to prevent the clinical consequences of SOS in an experimental rat model. It is of importance to translate this observation to man in order to improve post-resectional outcomes of patients with CLMs treated with oxaliplatin-based neoadjuvant chemotherapy. 


\section{REFERENCES}

1. Clavien PA, Petrowsky H, DeOliveira ML, Graf R. Strategies for safer liver surgery and partial liver transplantation. N Engl J Med 2007; 356: 1545-59.

2. Dimick JB, Wainess RM, Cowan JA, Upchurch GR, Jr., Knol JA, Colletti LM. National trends in the use and outcomes of hepatic resection. J Am Coll Surg 2004; 199: 31-8.

3. Poon RT, Fan ST, Lo CM, Liu CL, Lam CM, Yuen WK, et al. Improving perioperative outcome expands the role of hepatectomy in management of benign and malignant hepatobiliary diseases: analysis of 1222 consecutive patients from a prospective database. Ann Surg 2004; 240: 698-708.

4. Ergina PL, Cook JA, Blazeby JM, Boutron I, Clavien PA, Reeves BC, et al. Challenges in evaluating surgical innovation. Lancet 2009; 374: 1097-104.

5. Kaido T. Analysis of randomized controlled trials on hepatopancreatic surgery. Dig Dis Sci 2006; 51: 1761-6.

6. Gluud C. The culture of designing hepato-biliary randomised trials. J Hepatol 2006; 44: 607-15.

7. Martin RC, 2nd, Brennan MF, Jaques DP. Quality of complication reporting in the surgical literature. Ann Surg 2002; 235: 803-13.

8. Guller U, Oertli D. Sample size matters: a guide for surgeons. World J Surg 2005; 29: 601-5.

9. Prentice RL. Surrogate endpoints in clinical trials: definition and operational criteria. Stat Med 1989; 8: 431-40.

10. Ferreira-Gonzalez I, Permanyer-Miralda G, Busse JW, Bryant DM, Montori VM, Alonso-Coello P, et al. Methodologic discussions for using and interpreting composite endpoints are limited, but still identify major concerns. J Clin Epidemiol 2007; 60: 651-7.

11. Freemantle N, Calvert M, Wood J, Eastaugh J, Griffin C. Composite outcomes in randomized trials: greater precision but with greater uncertainty? Jama 2003; 289: 2554-9.

12. Ferreira-Gonzalez I, Busse JW, Heels-Ansdell D, Montori VM, AkI EA, Bryant DM, et al. Problems with use of composite end points in cardiovascular trials: systematic review of randomised controlled trials. Bmj 2007; 334: 786-92.

13. Bethel MA, Holman R, Haffner SM, Califf RM, Huntsman-Labed A, Hua TA, et al. Determining the most appropriate components for a composite clinical trial outcome. Am Heart J 2008; 156: 63340.

14. Clavien PA, Barkun J, de Oliveira ML, Vauthey JN, Dindo D, Schulick RD, et al. The Clavien-Dindo classification of surgical complications: five-year experience. Ann Surg 2009; 250: 187-96.

15. Strasberg SM, Linehan DC, Hawkins WG. The accordion severity grading system of surgical complications. Ann Surg 2009; 250: 177-86.

16. Imamura H, Seyama Y, Kokudo N, Maema A, Sugawara Y, Sano K, et al. One thousand fifty-six hepatectomies without mortality in 8 years. Arch Surg 2003; 138: 1198-206.

17. Kooby DA, Stockman J, Ben-Porat L, Gonen M, Jarnagin WR, Dematteo RP, et al. Influence of transfusions on perioperative and long-term outcome in patients following hepatic resection for colorectal metastases. Ann Surg 2003; 237: 860-9.

18. Gomez D, Morris-Stiff G, Wyatt J, Toogood GJ, Lodge JP, Prasad KR. Surgical technique and systemic inflammation influences long-term disease-free survival following hepatic resection for colorectal metastasis. J Surg Oncol 2008; 98: 371-6.

19. Pringle JH. V. Notes on the Arrest of Hepatic Hemorrhage Due to Trauma. Ann Surg 1908; 48: 541-9. 
20. Rahbari NN, Wente MN, Schemmer P, Diener MK, Hoffmann K, Motschall E, et al. Systematic review and meta-analysis of the effect of portal triad clamping on outcome after hepatic resection. $\mathrm{Br} J$ Surg 2008; 95: 424-32.

21. Lai EC, Fan ST, Lo CM, Chu KM, Liu C. Anterior approach for difficult major right hepatectomy. World J Surg 1996; 20: 314-18.

22. Fan ST. Protection of the liver during partial hepatectomy. Hepatobiliary Pancreat Dis Int 2004; 3: 490-4.

23. van de Poll MC, Derikx JP, Buurman WA, Peters WH, Roelofs HM, Wigmore SJ, et al. Liver manipulation causes hepatocyte injury and precedes systemic inflammation in patients undergoing liver resection. World J Surg 2007; 31: 2033-8.

24. Schemmer P, Connor HD, Arteel GE, Raleigh JA, Bunzendahl H, Mason RP, et al. Reperfusion injury in livers due to gentle in situ organ manipulation during harvest involves hypoxia and free radicals. J Pharmacol Exp Ther 1999; 290: 235-40.

25. The FO, Bennink RJ, Ankum WM, Buist MR, Busch OR, Gouma DJ, et al. Intestinal handling-induced mast cell activation and inflammation in human postoperative ileus. Gut 2008; 57: 33-40.

26. Belghiti J, Guevara OA, Noun R, Saldinger PF, Kianmanesh R. Liver hanging maneuver: a safe approach to right hepatectomy without liver mobilization. J Am Coll Surg 2001; 193: 109-11.

27. The FO, Buist MR, Lei A, Bennink RJ, Hofland J, van den Wijngaard RM, et al. The role of mast cell stabilization in treatment of postoperative ileus: a pilot study. Am J Gastroenterol 2009; 104 : 2257-66.

28. Benzoni E, Lorenzin D, Baccarani U, Adani GL, Favero A, Cojutti A, et al. Resective surgery for liver tumor: a multivariate analysis of causes and risk factors linked to postoperative complications. Hepatobiliary Pancreat Dis Int 2006; 5: 526-33.

29. Esaki M, Sano T, Shimada K, Sakamoto Y, Takahashi Y, Wakai K, et al. Randomized clinical trial of hepatectomy using intermittent pedicle occlusion with ischaemic intervals of 15 versus 30 minutes. Br J Surg 2006; 93: 944-51.

30. Kim YI, Fujita S, Hwang YJ, Chun JM, Song KE, Chun BY. Successful intermittent application of the Pringle maneuver for 30 minutes during human hepatectomy: a clinical randomized study with use of a protease inhibitor. Hepatogastroenterology 2007; 54: 2055-60.

31. Sugiyama Y, Ishizaki Y, Imamura H, Sugo H, Yoshimoto J, Kawasaki S. Effects of intermittent Pringle's manoeuvre on cirrhotic compared with normal liver. Br J Surg 2010; 97: 1062-9.

32. Glatz JF, van der Vusse GJ. Cellular fatty acid-binding proteins: their function and physiological significance. Prog Lipid Res 1996; 35: 243-82.

33. Storch J, McDermott L. Structural and functional analysis of fatty acid-binding proteins. J Lipid Res 2009; 50 Suppl: S126-31.

34. Pelsers MM, Hermens WT, Glatz JF. Fatty acid-binding proteins as plasma markers of tissue injury. Clin Chim Acta 2005; 352: 15-35.

35. Dello SA, Reisinger KW, van Dam RM, Bemelmans MH, van Kuppevelt TH, van den Broek MA, et al. Total intermittent Pringle maneuver during liver resection can induce intestinal epithelial cell damage and endotoxemia. PLoS One 2012; 7: e30539.

36. Wicherts DA, de Haas RJ, Adam R. Bringing unresectable liver disease to resection with curative intent. Eur J Surg Oncol 2007; 33 Suppl 2: S42-51.

37. Adam R, Delvart V, Pascal G, Valeanu A, Castaing D, Azoulay D, et al. Rescue surgery for unresectable colorectal liver metastases downstaged by chemotherapy: a model to predict long-term survival. Ann Surg 2004; 240: 644-57. 
38. Nordlinger B, Sorbye H, Glimelius B, Poston GJ, Schlag PM, Rougier P, et al. Perioperative chemotherapy with FOLFOX4 and surgery versus surgery alone for resectable liver metastases from colorectal cancer (EORTC Intergroup trial 40983): a randomised controlled trial. Lancet 2008; 371: 1007-16.

39. DeLeve LD. Hepatic microvasculature in liver injury. Semin Liver Dis 2007; 27: 390-400.

40. Rubbia-Brandt L, Lauwers GY, Wang H, Majno PE, Tanabe K, Zhu AX, et al. Sinusoidal obstruction syndrome and nodular regenerative hyperplasia are frequent oxaliplatin-associated liver lesions and partially prevented by bevacizumab in patients with hepatic colorectal metastasis. Histopathology 2010; 56: 430-9.

41. Soubrane O, Brouquet A, Zalinski S, Terris B, Brezault C, Mallet V, et al. Predicting high grade lesions of sinusoidal obstruction syndrome related to oxaliplatin-based chemotherapy for colorectal liver metastases: correlation with post-hepatectomy outcome. Ann Surg 2010; 251: 454-60.

42. Karoui M, Penna C, Amin-Hashem M, Mitry E, Benoist S, Franc B, et al. Influence of preoperative chemotherapy on the risk of major hepatectomy for colorectal liver metastases. Ann Surg 2006; 243: 1-7.

43. Narita M, Oussoultzoglou E, Chenard MP, Rosso E, Casnedi S, Pessaux P, et al. Sinusoidal obstruction syndrome compromises liver regeneration in patients undergoing two-stage hepatectomy with portal vein embolization. Surg Today 2011; 41: 7-17.

44. Rubbia-Brandt L, Audard V, Sartoretti P, Roth AD, Brezault C, Le Charpentier M, et al. Severe hepatic sinusoidal obstruction associated with oxaliplatin-based chemotherapy in patients with metastatic colorectal cancer. Ann Oncol 2004; 15: 460-6.

45. Overman MJ, Maru DM, Charnsangavej C, Loyer EM, Wang H, Pathak P, et al. Oxaliplatin-mediated increase in spleen size as a biomarker for the development of hepatic sinusoidal injury. J Clin Oncol 2010; 28: 2549-55.

46. Nakano H, Oussoultzoglou E, Rosso E, Casnedi S, Chenard-Neu MP, Dufour P, et al. Sinusoidal Injury Increases Morbidity After Major Hepatectomy in Patients With Colorectal Liver Metastases Receiving Preoperative Chemotherapy. Ann Surg 2008; 247: 118-124.

47. DeLeve LD, Valla DC, Garcia-Tsao G. Vascular disorders of the liver. Hepatology 2009; 49: 1729-64.

48. Eriksson S, Fraser JR, Laurent TC, Pertoft H, Smedsrod B. Endothelial cells are a site of uptake and degradation of hyaluronic acid in the liver. Exp Cell Res 1983; 144: 223-8.

49. Fraser JR, Laurent TC, Laurent UB. Hyaluronan: its nature, distribution, functions and turnover. $J$ Intern Med 1997; 242: 27-33.

50. Tamandl D, Klinger M, Eipeldauer S, Herberger B, Kaczirek K, Gruenberger B, et al. Sinusoidal obstruction syndrome impairs long-term outcome of colorectal liver metastases treated with resection after neoadjuvant chemotherapy. Ann Surg Oncol 2010; 18: 421-30.

51. Rubbia-Brandt L, Giostra E, Brezault C, Roth AD, Andres A, Audard V, et al. Importance of histological tumor response assessment in predicting the outcome in patients with colorectal liver metastases treated with neo-adjuvant chemotherapy followed by liver surgery. Ann Oncol 2007; 18: 299-304.

52. Mandard AM, Dalibard F, Mandard JC, Marnay J, Henry-Amar M, Petiot JF, et al. Pathologic assessment of tumor regression after preoperative chemoradiotherapy of esophageal carcinoma. Clinicopathologic correlations. Cancer 1994; 73: 2680-6.

53. Allen M, Louise Jones J. Jekyll and Hyde: the role of the microenvironment on the progression of cancer. J Pathol 2010; 223: 162-76.

54. DeLeve LD, McCuskey RS, Wang X, Hu L, McCuskey MK, Epstein RB, et al. Characterization of a reproducible rat model of hepatic veno-occlusive disease. Hepatology 1999; 29: 1779-91. 
55. Bast A, Haenen GR, Bruynzeel AM, Van der Vijgh WJ. Protection by flavonoids against anthracycline cardiotoxicity: from chemistry to clinical trials. Cardiovasc Toxicol 2007; 7: 154-9.

56. Wadworth AN, Faulds D. Hydroxyethylrutosides. A review of its pharmacology, and therapeutic efficacy in venous insufficiency and related disorders. Drugs 1992; 44: 1013-32. 
Chapter 12

Summary and discussion (in Dutch) 

Een leveroperatie is de behandeling van keuze voor een groot aantal tumoren van de lever. De veiligheid van een leveroperatie is het afgelopen decennium toegenomen door vooruitgang op het gebied van patiëntenselectie, chirurgische techniek en postoperatieve zorg ${ }^{1-3}$. Door deze toegenomen veiligheid komen steeds meer patiënten met levertumoren in aanmerking voor een operatie.

Bij een leveroperatie wordt het deel van de lever dat tumor bevat, verwijderd. Zowel het volume als de functie van het overgebleven deel van de lever (de restlever) zijn na zo'n operatie tijdelijk afgenomen. Wanneer zij niet tijdig herstellen, kan postoperatief leverfalen (PLF) optreden. PLF wordt gekenmerkt door een verstoring van de fysiologische functies van de lever. Dit kan leiden tot een ophoping van giftige stoffen in het bloed en een verminderde aanmaak van essentiële eiwitten. In de inleiding van dit proefschrift (hoofdstuk 1) worden verschillende aspecten van PLF beschreven. Kort samengevat, ontwikkelt ongeveer één op de dertig patiënten symptomen van leverfalen na een leveroperatie. Risicofactoren voor het ontwikkelen van PLF zijn operatie- en patiëntgebonden. Leverfalen is de nummer één doodsoorzaak na een leveroperatie. Omdat er tot op heden geen goede behandelopties zijn, is het letterlijk van levensbelang om PLF te voorkomen.

Dit proefschrift heeft als doel de uitkomsten van leverchirurgie te analyseren en, waar mogelijk, te verbeteren. Specifieke aandacht wordt hierbij gegeven aan de risicofactoren voor het ontwikkelen van PLF. Dit vertaalt zich in de volgende drie doelen:

Doel 1. Ontwikkelen van methoden die de haalbaarheid vergroten van het verrichten van kwalitatief hoogwaardig onderzoek naar klinische uitkomsten van leverchirurgie (hoofdstuk 2, 3 en 4).

Doel 2. Evalueren van operatiegebonden risicofactoren voor PLF middels het bestuderen van het effect van twee verschillende chirurgische technieken op het ontwikkelen van levercel schade (hoofdstuk 5 en 6).

Doel 3. In kaart brengen van patiëntgebonden risicofactoren voor PLF, in het bijzonder schade aan de haarvaten van de lever als gevolg van chemotherapie in patiënten met leveruitzaaiingen van darmkanker (hoofdstuk 7, 8, 9 en 10).

\section{DEEL I. ANALYSE VAN UITKOMSTEN VAN LEVERCHIRURGIE: KRITISCHE BESCHOUWING VAN HUIDIGE KLINISCHE EINDPUNTEN}

Het eerste doel van dit proefschrift is het ontwikkelen van strategieën die de haalbaarheid vergroten van het verrichten van gerandomiseerd onderzoek naar klinische uitkomsten van leverchirurgie.

Geneeskunde op basis van wetenschappelijk bewijs, ook wel evidence-based medicine genoemd, is de gouden standaard in de hedendaagse patiëntenzorg. Richtlijnen voor 
de zorg van chirurgische patiënten zijn bij voorkeur gebaseerd op resultaten van kwalitatief hoogwaardig onderzoek, zoals gerandomiseerde studies en meta-analyses. Op het gebied van de leverchirurgie is het afgelopen decennium slechts een beperkt aantal kwalitatief hoogwaardige studies uitgevoerd ${ }^{4}$. Een verklaring voor dit kleine aantal studies zou kunnen zijn dat het uitvoeren van gerandomiseerd onderzoek binnen de leverchirurgie uitdagend is ${ }^{5}$. Deze uitdaging is zowel praktisch als technisch van aard, zoals het verzamelen van voldoende proefpersonen binnen een acceptabele termijn en de selectie van een eindpunt dat de onderzoeksvraag betrouwbaar kan beantwoorden $^{6}$. Traditioneel worden binnen de chirurgie klinische eindpunten, zoals overlijden (mortaliteit) of postoperatieve complicaties (morbiditeit), gekozen om het effect van een interventie te beoordelen ${ }^{7}$. Daarom focust deel I van dit proefschrift zich op het vergroten van de haalbaarheid van gerandomiseerd onderzoek dat gebruik maakt van klinische eindpunten van leverchirurgie.

In hoofdstuk $\mathbf{2}$ werd de haalbaarheid van het verrichten van een gerandomiseerde studie binnen de leverchirurgie met een klinisch eindpunt als primair eindpunt geëvalueerd. Op basis van literatuuronderzoek werd het gemiddelde voorkomen (incidentie) van mortaliteit en morbiditeit na een leveroperatie berekend in de periode tussen 2002 en 2007. De gemiddelde mortaliteit was 1.0 procent en de gemiddelde morbiditeit 28.9 procent; PLF had een incidentie van 2.6 procent. De groepsgrootte voor een gerandomiseerde studie werd berekend op basis van de gevonden incidentiegetallen. In het algemeen geldt dat de groepsgrootte die nodig is voor een studie groter is, naarmate de incidentie van het eindpunt dat bestudeerd wordt, lager is ${ }^{8}$. We berekenden dat er ongeveer 12000 patiënten nodig zouden zijn voor een studie die tot doel had een één derde vermindering van de incidentie van PLF aan te tonen. Omdat in alle Nederlandse ziekenhuizen samen jaarlijks slechts 500 leveroperaties worden uitgevoerd, concludeerden wij dat de haalbaarheid van het verrichten van een gerandomiseerde studie met een klinisch eindpunt zoals PLF minimaal is. Wij stellen voor om samengestelde eindpunten te gebruiken, multicentrische studies op te zetten of meta-analyses uit te voeren om de haalbaarheid van kwalitatief hoogwaardig onderzoek dat gebruik maakt van klinische eindpunten na leverchirurgie te vergroten. Als alternatief zouden surrogaat eindpunten gebruikt kunnen worden, echter robuuste validatie van deze eindpunten binnen de leverchirurgie ontbreekt tot op heden.

Voor het gebruik van de eerder genoemde samengestelde eindpunten of het verrichten van meta-analyses zijn uniforme definities van klinische eindpunten noodzakelijk ${ }^{10}$. Dit is belangrijk omdat anders het risico bestaat dat twee totaal verschillende condities met elkaar vergeleken worden. In hoofdstuk $\mathbf{3}$ werden uniforme definities van klinische eindpunten na leverchirurgie geformuleerd. Middels een tweede literatuuronderzoek ontdekten we dat duidelijke definities van klinische uitkomsten, die gebruikt werden als eindpunten van gerandomiseerde studies in de leverchirurgie, in minder dan één derde 
van de artikelen werden beschreven. Als zij beschreven werden, bleken de definities van eenzelfde klinische uitkomstmaat substantieel te verschillen tussen artikelen. De definities die we tijdens het literatuuronderzoek vonden, vormden de basis van een elektronische enquête. Deze enquête werd verspreid onder 54 internationaal vooraanstaande leverchirurgen in een poging consensus te bereiken over de definitie van de meest gebruikte klinische eindpunten. De enquête werd door 31 chirurgen ingevuld (respons percentage van 57 procent) en leidde tot aanpassingen van de voorgestelde definities op basis van het commentaar van de respondenten. Zo ontstonden er gestandaardiseerde definities van de meest frequent gebruikte klinische eindpunten van leverchirurgie. Voordat deze definities wereldwijd geïmplementeerd kunnen worden, moeten zij door een nog grotere groep van experts worden beoordeeld en in een grote patiëntengroep worden getest (validatie).

Vooruitlopend op deze validatie, werden de geformuleerde definities alvast gebruikt in hoofdstuk 4 om een leverchirurgie specifiek samengesteld eindpunt te ontwikkelen. Een samengesteld eindpunt is een combinatie van twee of meer klinische uitkomstmaten, die als één eindpunt worden beschouwd ${ }^{11}$. Samengestelde eindpunten leiden tot een groter onderscheidingsvermogen (power) en efficiëntie, omdat zij een hogere incidentie hebben dan een enkelvoudig klinisch eindpunt. Dientengevolge zal de groepsgrootte van een studie die een samengesteld eindpunt als primair eindpunt gebruikt, kleiner zijn. Op basis van voorgenoemde elektronische enquête werd een goed gedefinieerd en zorgvuldig gecomponeerd leverchirurgie specifiek samengestelde eindpunt ontwikkeld, bestaand uit de individuele componenten buikwaterzucht (ascites), PLF, gallekkage, intra-abdominale bloeding, intra-abdominaal abces en mortaliteit. Eén van de voorwaarden voor de betrouwbaarheid van een samengesteld eindpunt is dat de incidentie van de componenten vergelijkbaar is ${ }^{12,13}$. Daarom werd de incidentie van de individuele componenten van het leverchirurgie specifiek samengestelde eindpunt geëvalueerd in 815 patiënten die een leveroperatie ondergingen in twee Europese levercentra. Het voorkomen van de individuele componenten varieerde bij benadering tussen de 1 en 10 procent. Het samengestelde eindpunt had een incidentie van 11 procent in het ene en 19 procent in het andere cohort. De verdubbeling van de incidentie van het leverchirurgie specifiek samengestelde eindpunt ten opzicht van de individuele componenten, leidde grofweg tot een halvering van de benodigde patiëntenaantallen in een gerandomiseerde studie. De bruikbaarheid van het leverchirurgie specifiek samengestelde eindpunt is dus veelbelovend, echter ook hier geldt dat aanvullende evaluatie gewenst is.

Samenvattend tonen de resultaten van deel I van dit proefschrift dat de haalbaarheid van gerandomiseerd onderzoek binnen de hedendaagse leverchirurgie, dat gebruik maakt van klinische eindpunten, laag is als gevolg van de grote patiëntenaantallen die nodig zijn voor voldoende power. De oplossing kan gezocht worden in het uitvoeren 
van meta-analyses of de implementatie van een goed gedefinieerd leverchirurgie specifiek samengesteld eindpunt dat bestaat uit ascites, PLF, gallekkage, intra-abdominale bloeding, intra-abdominaal abces en operatieve mortaliteit. Aanvullende evaluatie van dit eindpunt is gewenst.

\section{DEEL II. ANALYSE VAN UITKOMSTEN VAN LEVERCHIRURGIE: LEVERCEL SCHADE ALS GEVOLG VAN CHIRURGISCHE TECHNIEK}

Het evalueren van het effect van verschillende chirurgische technieken op levercel schade in patiënten die een leveroperatie ondergaan, is het tweede doel van dit proefschrift.

Grote hoeveelheden bloedverlies tijdens een leveroperatie zijn geassocieerd met nadelige korte en lange termijn uitkomsten ${ }^{14-16}$. Zowel het mobiliseren van de lever als het afwisselend afklemmen en openzetten van de bloedtoevoer naar de lever (intermitterende Pringle manoeuvre) worden toegepast om het bloedverlies tijdens een leveroperatie te beperken. Het gebruik van deze technieken staat ter discussie, omdat ze beide zelf ook weer aanzienlijke levercel schade veroorzaken, hetgeen tot PLF zou kunnen leiden ${ }^{17-19}$. Om deze levercel schade beter te karakteriseren, worden in deel II van dit proefschrift beide technieken afzonderlijk bestudeerd in patiënten die een leveroperatie ondergaan.

Allereerst het mobiliseren van de lever: dit omvat het losmaken van de lever van de omgevende structuren zoals de buikwand, het middenrif en de onderste holle ader. Het doel van het mobiliseren van de lever tijdens een leveroperatie is het bloedverlies uit aders die vanuit de lever naar deze omgevende structuren lopen, te controleren. Onze onderzoeksgroep toonde eerder al aan dat mobilisatie van de lever de belangrijkste oorzaak van levercel schade tijdens een leveroperatie was. Tevens lieten we al zien dat het mobiliseren van de lever gevolgd werd door een ontstekingsreactie in het gehele lichaam ${ }^{20}$. Proefdiermodellen suggereren dat stimulatie van het immuunsysteem in de lever een belangrijke rol speelt bij het ontwikkelen van mobilisatie-geassocieerde levercel schade ${ }^{21}$. Echter, in de mens is deze relatie nog nooit aangetoond. Het is belangrijk om meer inzicht te krijgen in rol van het immuunsysteem bij het ontstaan van levercel schade door mobilisatie, omdat de ontstekingsreactie beïnvloed kan worden door bijvoorbeeld ontstekingsremmende medicatie. Daarom werd in hoofdstuk $\mathbf{5}$ de link tussen mobilisatie van de lever, levercel schade en ontsteking in de lever onderzocht bij 25 patiënten die een leveroperatie ondergingen. Wij toonden aan dat er een verband was tussen mobilisatie van de lever en ontsteking in de lever. Deze ontsteking werd gekenmerkt door de aanwezigheid van een groot aantal ontstekingscellen in de lever en een verhoogde expressie van genen die betrokken zijn bij de productie van ontstekingseiwitten. Tevens vonden wij een significante relatie tussen de duur van de mobilisatie en 
het aantal ontstekingscellen dat zich in de lever ophoopte. De groepsgrootte van deze studie was te klein om een relatie tussen mobilisatie-geïnduceerde levercel schade en klinische eindpunten van leverchirurgie vast te stellen. Andere onderzoekers lieten echter al zien dat het voorkomen van PLF de neiging had lager te zijn in patiënten die een leveroperatie ondergingen via de zogenaamde anterieure benadering ${ }^{22}$. De anterieure benadering wordt ook wel de no touch techniek genoemd, omdat bij deze techniek de lever niet geheel wordt losgemaakt uit zijn omgeving. Op basis van voorgenoemde data veronderstellen wij dat het klinisch relevant zou kunnen zijn om levercel schade door mobilisatie te voorkomen. Preventie zou gelegen kunnen zijn in het toepassen van de eerdergenoemde anterieure benadering of het toedienen van ontstekingsremmende medicatie tijdens een leveroperatie 18, 23-25. $^{\text {. }}$

$\mathrm{Na}$ het mobiliseren van de lever volgt in de regel het doorsnijden van het leverweefsel om de levertumor te verwijderen. Een van de manieren om het bloedverlies tijdens het doornemen van het leverweefsel te verminderen, is de intermitterende Pringle manoeuvre ${ }^{26}$. De intermitterende Pringle manoeuvre behelst het afwisselend afklemmen en openzetten van de bloedtoevoer naar de lever. De bloedtoevoer naar de lever wordt verzorgd door twee aders: de leverslagader, die zuurstofrijk bloed direct uit de grote lichaamsslagader naar de lever vervoert, en de poortader, die zuurstofarm bloed uit de darm naar de lever vervoert. Tijdens de intermitterende Pringle manoeuvre worden beide aders afgesloten en wordt de lever een periode onvoldoende van bloed voorzien (ischemie), waarna de bloedtoevoer weer wordt hersteld (reperfusie). De optimale duur van de ischemische intervallen tijdens de Pringle manoeuvre staat nog ter discussie. Gewoonlijk wordt het effect van verschillende ischemische intervallen tijdens de Pringle manoeuvre bestudeerd op basis van het gehalte leverenzymen in het bloed op verschillende dagen na een leveroperatie. Het is echter maar zeer de vraag of deze markers wel gevoelig genoeg zijn om klinisch relevante verschillen in levercel schade als het gevolg van de intermitterende Pringle manoeuvre te detecteren ${ }^{27}$. Het intra-operatief monitoren van de plasma concentratie van het meer specifieke eiwit liver fatty acidbinding protein (L-FABP) is mogelijk een meer accurate manier om verschillen in levercel schade te bepalen. L-FAPB is een klein eiwit met een lage molecuul massa dat in de cel betrokken is bij het transport en metabolisme van vetten ${ }^{28,29}$. Na leverschade stijgt het L-FABP gehalte in het bloed vlug omdat L-FAPB snel wordt vrijgemaakt uit beschadigde levercellen ${ }^{30}$. In hoofdstuk 6 toonden we in een gerandomiseerde studie bij de mens aan dat een Pringle manoeuvre met 30 minuten durende ischemische intervallen leidde tot vergelijkbare levercel schade, gebaseerd op het L-FABP gehalte in het bloed, als een Pringle manoeuvre met 15 minuten durende ischemische intervallen. Daarbij was ook de postoperatieve leverfunctie gelijkwaardig tussen de twee groepen. Bovendien deden we de belangrijke bevinding dat het niet toepassen van de Pringle manoeuvre leidde tot significant minder levercel schade. Er wordt vaak gesuggereerd dat de Pringle 
manoeuvre kan leiden tot darmschade als gevolg van het afklemmen van de poortader, echter hiervoor vonden wij in deze studie geen bewijs. In een follow-up studie van onze onderzoeksgroep vonden we echter wel aanwijzingen voor een verhoogde aanwezigheid van bacteriële producten in het bloed na een Pringle manoeuvre, hetgeen zou kunnen wijzen op een verstoring van de darm barrière ${ }^{31}$. Omdat in een recente meta-analyse geen klinisch voordeel werd gevonden van het gebruik van de Pringle manoeuvre ${ }^{17}$, adviseren wij in principe geen Pringle manoeuvre te gebruiken tijdens het doorsnijden van het leverweefsel. Wanneer het noodzakelijk is om een Pringle manoeuvre te gebruiken, kunnen 30 minuten durende ischemische intervallen worden gekozen.

Concluderend toont deel II van dit proefschrift dat mobilisatie van de lever geassocieerd is met levercel schade en ontsteking in de lever, proportioneel aan de duur van de mobilisatie. Of het verminderen van de levercel schade of de ontstekingsreactie bij de mens gunstige effecten heeft, moet onderzocht worden in toekomstige studies. Daarnaast laten we zien dat een Pringle manoeuvre met 15 of 30 minuten durende ischemische intervallen leidt tot vergelijkbare levercel schade en postoperatieve leverfunctie. Echter, het niet toepassen van de Pringle manoeuvre leidt tot significant minder schade en daarom zou de Pringle manoeuvre terughoudend gebruikt moeten worden tijdens leverchirurgie.

\section{DEEL III. ANALYSE VAN UITKOMSTEN VAN LEVERCHIRURGIE: LEVERCEL SCHADE ALS GEVOLG VAN CHEMOTHERAPIE}

Het derde doel van dit proefschrift is het bestuderen van vaatschade aan de lever als gevolg van chemotherapie in patiënten met leveruitzaaiingen van darmkanker.

Voor patiënten met leveruitzaaiingen van darmkanker, ook wel colorectale levermetastasen (CLMs) genoemd, is een operatie aan de lever, waarbij alle uitzaaiingen worden verwijderd, de enige behandeling met kans op genezing ${ }^{32}$. Helaas komt slechts de minderheid van patiënten met CLMs in eerste instantie in aanmerking voor een operatie, omdat de uitzaaiingen zich in de gehele lever of zelfs daarbuiten bevinden. Daarom is een multidisciplinaire behandeling bestaande uit chemotherapie met capecitabine, oxaliplatin of irinotecan gevolgd door een leveroperatie, in deze groep tegenwoordig meer regel dan uitzondering. Bij 15 tot 30 procent van de patiënten met uitgebreide leveruitzaaiingen leidt behandeling met chemotherapie tot een verkleining van de tumormassa, waardoor een leveroperatie alsnog mogelijk wordt ${ }^{33}$. Het veelgebruikte chemotherapeuticum oxaliplatin kent echter serieuze bijwerkingen. Een van deze bijwerkingen is schade aan de haarvaten (sinusoïden) van de lever. Deze schade staat bekend als het sinusoïdaal obstructie syndroom $(\mathrm{SOS})^{34-36}$. De aanwezigheid van SOS is geassocieerd met een verhoogde kans op complicaties na een uitgebreide leveropera- 
tie, zoals het ontwikkelen van en overlijden aan $\mathrm{PLF}^{36-38}$. In deel III van dit proefschrift bestuderen we daarom de effecten van oxaliplatin op de haarvaten van de lever.

In hoofdstuk 7 lieten we aan de hand van twee casussen zien waartoe de aanwezigheid van sinusoïdale schade als gevolg van oxaliplatin kan leiden in patiënten die een uitgebreide leveroperatie ondergingen. Beide patiënten hadden geen onderliggende leverziekte en werden in verband met leveruitzaaiingen van darmkanker behandeld met oxaliplatin voor de leveroperatie. Analyse van het leverweefsel na de operatie toonde uitgebreide sinusoïdale schade. Door deze schade was de doorstroming van het bloed in de lever verstoord, hetgeen leidde tot een verhoogde druk in de poortader (portale hypertensie) ${ }^{39}$. Portale hypertensie is een zorgwekkend ziektebeeld, omdat dit tot levensbedreigende complicaties zoals maag- en slokdarmbloedingen kan leiden. Deze complicaties traden bij beide patiënten op en leidden tot het overlijden van één van hen. Hoewel in dit hoofdstuk het klinische beloop van slechts twee patiënten wordt beschreven, is het probleem van sinusoïdale schade na oxaliplatin groot. SOS ontstaat in ongeveer twee tot zeven op de tien patiënten die met oxaliplatin worden behandeld wegens leveruitzaaiingen ${ }^{35,36,38,40}$. Gezien de schade die de behandeling met oxaliplatin met zich meebrengt, is het belangrijk het voordeel van het gebruik van oxaliplatin per patiënt af te zetten tegen het risico op het ontwikkelen van sinusoïdale schade. Op basis van deze afweging zou bepaald moeten worden of het gebruik van oxaliplatin voor een leveroperatie daadwerkelijk noodzakelijk is. Als dit het geval is, zoals bij patiënten die in eerste instantie niet in aanmerking komen voor een leveroperatie, is het van belang het ontstaan van deze schade vroegtijdig te herkennen en zo mogelijk te voorkomen.

Vroegtijdige herkenning van sinusoïdale schade als gevolg van oxaliplatin is belangrijk, omdat het operatieplan hierdoor kan veranderen. Patiënten met SOS ondergaan bij voorkeur een minder uitgebreide operatie of een operatie in twee stappen, omdat de functie van de restlever is aangetast. De huidige detectiemethoden voor SOS maken gebruik van indirecte markers voor vaatschade en hebben daarom een bescheiden sensitiviteit en specificiteit ${ }^{36,38,40}$. In hoofdstuk 8 werd bestudeerd of het meten van het hyaluronzuur $(\mathrm{HZ})$ gehalte in het bloed een meer betrouwbare maat zou kunnen zijn voor het ontdekken van SOS in patiënten die behandeld zijn met oxaliplatin. $\mathrm{HZ}$ wordt in onze gewrichten geproduceerd. $\mathrm{Na}$ afgifte van $\mathrm{HZ}$ aan de bloedbaan wordt het vrijwel volledig opgenomen en verwerkt door de sinusoïdale cellen (SCs) in de haarvaten van de lever ${ }^{41}{ }^{42}$. Derhalve wordt het $\mathrm{HZ}$ gehalte in het bloed gezien als maat voor de functie van deze SCs. Juist deze SCs zijn beschadigd in patiënten met SOS en dus veronderstelden wij dat het $\mathrm{HZ}$ gehalte in het bloed verhoogd zou zijn in deze groep. In patiënten die tussen 2008 en 2009 in het Maastricht Universitair Medisch Centrum een leveroperatie ondergingen na behandeling met oxaliplatin, toonde het leverweefsel in 58 procent tekenen van SOS. Het $\mathrm{HZ}$ gehalte van het bloed was significant verhoogd in patiënten met SOS in vergelijking met patiënten zonder SOS. Bij een grenswaarde van 
$44.1 \mathrm{ng} / \mathrm{mL}$ had $\mathrm{HZ}$ een sensitiviteit van 67 procent en een specificiteit van 83 procent voor de detectie van SOS. De negatief voorspellende waarde was 91 procent. Op basis van deze getallen concludeerden we dat de kans op sinusoïdale schade heel klein is, als het $\mathrm{HZ}$ gehalte onder de grenswaarde van $44.1 \mathrm{ng} / \mathrm{mL}$ ligt. Dit houdt concreet in dat aanvullend onderzoek naar de aanwezigheid van SOS niet noodzakelijk is in deze patiënten. Aanvullend onderzoek is wel aangewezen in patiënten met een $\mathrm{HZ}$ gehalte boven de grenswaarde van $44.1 \mathrm{ng} / \mathrm{mL}$. Dit aanvullend onderzoek zou bijvoorbeeld kunnen bestaan uit het verkrijgen van leverweefsel via een biopsie. Of patiënten met een verhoogde $\mathrm{HZ}$ concentratie uiteindelijk baat hebben bij een beperktere leveroperatie, moet in de toekomst blijken. Om een verklaring te vinden voor het verhoogde $\mathrm{HZ}$ gehalte in het bloed bij patiënten met SOS, voerden we een deelstudie uit. Een verhoogde $\mathrm{HZ}$ waarde moet namelijk het gevolg zijn van ofwel een verhoogde aanmaak van $\mathrm{HZ}$ ofwel een verminderde afbraak van HZ. Verrassend genoeg vonden we geen bewijs voor een verminderde afbraak van $\mathrm{HZ}$ door de beschadigde SCs in de lever van patiënten met SOS.

Een recente studie laat zien dat de lange termijn overleving van patiënten met SOS verminderd is ten opzichte van patiënten zonder SOS na oxaliplatin-bevattende chemotherapie wegens leveruitzaaiingen van darmkanker ${ }^{43}$. De lange termijn overleving van patiënten met leveruitzaaiingen wordt onder andere bepaald door de reactie van deze uitzaaiingen op chemotherapie ${ }^{44}$. Deze reactie wordt weergegeven als de tumor regressie graad (TRG) ${ }^{45}$. De TRG wordt bepaald door het aantal vitale tumorcellen in leveruitzaaiingen onder de microscoop te bestuderen. Wij veronderstelden dat de tumor respons in patiënten met SOS afgenomen zou zijn, hetgeen de verminderde lange termijn overleving zou kunnen verklaren. In hoofdstuk 9 leverden we inderdaad het bewijs dat de aanwezigheid van SOS leidde tot een verminderde tumor respons op oxaliplatin. Een verklaring voor de afname van het celdodende effect van oxaliplatin in patiënten met SOS zou kunnen zijn dat de vaatschade in de lever leidt tot een ongunstig tumor milieu en een verminderde concentratie van het chemotherapeuticum op de plaats van de leveruitzaaiing. Helaas konden we in deze studie (nog) geen relatie met de lange termijn overleving leggen omdat onze patiënten tussen 2008 en 2009 werden geopereerd. Wanneer de 5-jaars follow-up is bereikt, is analyse van het effect van SOS en tumor regressie graad op de ziektevrije en totale overleving een belangrijke volgende stap om onze hypothese te bevestigen.

In de ontwikkeling van SOS spelen oxidatieve stress, ontsteking en vaatschade een belangrijke rol ${ }^{34}$. Deze processen zijn dan ook belangrijke therapeutische aanknopingspunten. In hoofdstuk 10 bestudeerden we het beschermende effect van het flavonoïde monoHER op het ontstaan van SOS in een proefdiermodel ${ }^{46}$. MonoHER bezit verschillende gunstige kenmerken, zoals een beschermend effect op de vaatwand en ontstekingsremmende en antioxidatieve eigenschappen ${ }^{47,48}$. Recent is reeds aangetoond dat 
monoHER beschermend werkt tegen chemotherapie-geïnduceerde hartcel schade, welke ook wordt veroorzaakt door oxidatieve stress ${ }^{47}$. In ons proefdiermodel was het flavonoïde monoHER in staat om de ontwikkeling van vaatschade, levercel schade en portale hypertensie te voorkomen. Tevens toonden we in celkweken aan dat monoHER geen effect had op de celdodende capaciteit van oxaliplatin. Aanvullende studies zijn nodig om de effectiviteit van monoHER te bestuderen in een proefdiermodel waarin een leveroperatie wordt uitgevoerd. Wanneer monoHER ook dan effectief blijkt, is een studie in de mens een logische volgende stap.

Samenvattend laten we in deel III van dit proefschrift zien dat vaatschade in de lever als gevolg van oxaliplatin kan leiden tot ongunstige korte termijn uitkomsten. Ook leveren we bewijs dat de respons van leveruitzaaiingen op oxaliplatin verminderd is in patiënten met SOS. Dit zou invloed kunnen hebben op de lange termijn uitkomsten van patiënten met CLMs en SOS, echter dit moet onderzocht worden in toekomstige studies. Het $\mathrm{HZ}$ gehalte in het bloed kan worden gebruikt om patiënten met een verhoogd risico op SOS preoperatief te identificeren. Of deze patiënten profijt hebben van een beperkte leveroperatie moet in de toekomst blijken. Tot slot laten we zien dat het flavonoïde monoHER in staat is om vaatschade en levercel schade in een proefdiermodel te voorkomen. Het is van groot belang deze observatie te vertalen naar de mens om de uitkomsten van leverchirurgie na oxaliplatin-bevattende chemotherapie te verbeteren. 


\section{REFERENTIES}

1. Clavien PA, Petrowsky H, DeOliveira ML, Graf R. Strategies for safer liver surgery and partial liver transplantation. N Engl J Med 2007; 356:1545-59.

2. Dimick JB, Wainess RM, Cowan JA, et al. National trends in the use and outcomes of hepatic resection. J Am Coll Surg 2004; 199:31-8.

3. Poon RT, Fan ST, Lo CM, et al. Improving perioperative outcome expands the role of hepatectomy in management of benign and malignant hepatobiliary diseases: analysis of 1222 consecutive patients from a prospective database. Ann Surg 2004; 240:698-708.

4. Kaido T. Recent randomized controlled trials in hepatectomy. Hepatogastroenterology 2007; 54:1825-30.

5. Ergina PL, Cook JA, Blazeby JM, et al. Challenges in evaluating surgical innovation. Lancet 2009; 374:1097-104.

6. Gluud C. The culture of designing hepato-biliary randomised trials. J Hepatol 2006; 44:607-15.

7. Martin RC, 2nd, Brennan MF, Jaques DP. Quality of complication reporting in the surgical literature. Ann Surg 2002; 235:803-13.

8. Guller U, Oertli D. Sample size matters: a guide for surgeons. World J Surg 2005; 29:601-5.

9. Prentice RL. Surrogate endpoints in clinical trials: definition and operational criteria. Stat Med 1989; 8:431-40.

10. Ferreira-Gonzalez I, Permanyer-Miralda G, Busse JW, et al. Methodologic discussions for using and interpreting composite endpoints are limited, but still identify major concerns. J Clin Epidemiol 2007; 60:651-7.

11. Freemantle N, Calvert M, Wood J, et al. Composite outcomes in randomized trials: greater precision but with greater uncertainty? Jama 2003; 289:2554-9.

12. Ferreira-Gonzalez I, Busse JW, Heels-Ansdell D, et al. Problems with use of composite end points in cardiovascular trials: systematic review of randomised controlled trials. Bmj 2007; 334:786-92.

13. Bethel MA, Holman R, Haffner SM, et al. Determining the most appropriate components for a composite clinical trial outcome. Am Heart J 2008; 156:633-40.

14. Imamura $\mathrm{H}$, Seyama $\mathrm{Y}$, Kokudo $\mathrm{N}$, et al. One thousand fifty-six hepatectomies without mortality in 8 years. Arch Surg 2003; 138:1198-206.

15. Kooby DA, Stockman J, Ben-Porat L, et al. Influence of transfusions on perioperative and longterm outcome in patients following hepatic resection for colorectal metastases. Ann Surg 2003; 237:860-9.

16. Gomez D, Morris-Stiff G, Wyatt J, et al. Surgical technique and systemic inflammation influences long-term disease-free survival following hepatic resection for colorectal metastasis. J Surg Oncol 2008; 98:371-6.

17. Rahbari NN, Wente MN, Schemmer P, et al. Systematic review and meta-analysis of the effect of portal triad clamping on outcome after hepatic resection. Br J Surg 2008; 95:424-32.

18. Lai EC, Fan ST, Lo CM, et al. Anterior approach for difficult major right hepatectomy. World J Surg 1996; 20:314-18.

19. Fan ST. Protection of the liver during partial hepatectomy. Hepatobiliary Pancreat Dis Int 2004; 3:490-4.

20. van de Poll MC, Derikx JP, Buurman WA, et al. Liver manipulation causes hepatocyte injury and precedes systemic inflammation in patients undergoing liver resection. World J Surg 2007; 31:2033-8 
21. Schemmer P, Connor HD, Arteel GE, et al. Reperfusion injury in livers due to gentle in situ organ manipulation during harvest involves hypoxia and free radicals. J Pharmacol Exp Ther 1999; 290:235-40.

22. Liu CL, Fan ST, Cheung ST, et al. Anterior approach versus conventional approach right hepatic resection for large hepatocellular carcinoma: a prospective randomized controlled study. Ann Surg 2006; 244:194-203.

23. The FO, Bennink RJ, Ankum WM, et al. Intestinal handling-induced mast cell activation and inflammation in human postoperative ileus. Gut 2008; 57:33-40.

24. Belghiti J, Guevara OA, Noun R, et al. Liver hanging maneuver: a safe approach to right hepatectomy without liver mobilization. J Am Coll Surg 2001; 193:109-11.

25. The FO, Buist MR, Lei A, et al. The role of mast cell stabilization in treatment of postoperative ileus: a pilot study. Am J Gastroenterol 2009; 104:2257-66.

26. Pringle JH. V. Notes on the Arrest of Hepatic Hemorrhage Due to Trauma. Ann Surg 1908; 48:541-9.

27. Sugiyama $Y$, Ishizaki $Y$, Imamura $H$, et al. Effects of intermittent Pringle's manoeuvre on cirrhotic compared with normal liver. Br J Surg 2010; 97:1062-9.

28. Glatz JF, van der Vusse GJ. Cellular fatty acid-binding proteins: their function and physiological significance. Prog Lipid Res 1996; 35:243-82.

29. Storch J, McDermott L. Structural and functional analysis of fatty acid-binding proteins. J Lipid Res 2009; 50 Suppl:S126-31.

30. Pelsers MM, Hermens WT, Glatz JF. Fatty acid-binding proteins as plasma markers of tissue injury. Clin Chim Acta 2005; 352:15-35.

31. Dello SA, Reisinger KW, van Dam RM, et al. Total intermittent Pringle maneuver during liver resection can induce intestinal epithelial cell damage and endotoxemia. PLoS One 2012; 7:e30539.

32. Wicherts DA, de Haas RJ, Adam R. Bringing unresectable liver disease to resection with curative intent. Eur J Surg Oncol 2007; 33 Suppl 2:S42-51.

33. Adam R, Delvart V, Pascal G, et al. Rescue surgery for unresectable colorectal liver metastases downstaged by chemotherapy: a model to predict long-term survival. Ann Surg 2004; 240:644-57.

34. DeLeve LD. Hepatic microvasculature in liver injury. Semin Liver Dis 2007; 27:390-400.

35. Rubbia-Brandt L, Lauwers GY, Wang H, et al. Sinusoidal obstruction syndrome and nodular regenerative hyperplasia are frequent oxaliplatin-associated liver lesions and partially prevented by bevacizumab in patients with hepatic colorectal metastasis. Histopathology 2010; 56:430-9.

36. Soubrane O, Brouquet A, Zalinski S, et al. Predicting high grade lesions of sinusoidal obstruction syndrome related to oxaliplatin-based chemotherapy for colorectal liver metastases: correlation with post-hepatectomy outcome. Ann Surg 2010; 251:454-60.

37. Karoui M, Penna C, Amin-Hashem M, et al. Influence of preoperative chemotherapy on the risk of major hepatectomy for colorectal liver metastases. Ann Surg 2006; 243:1-7.

38. Nakano H, Oussoultzoglou E, Rosso E, et al. Sinusoidal Injury Increases Morbidity After Major Hepatectomy in Patients With Colorectal Liver Metastases Receiving Preoperative Chemotherapy. Ann Surg 2008; 247:118-124.

39. Wanless IR. Micronodular transformation (nodular regenerative hyperplasia) of the liver: a report of 64 cases among 2,500 autopsies and a new classification of benign hepatocellular nodules. Hepatology 1990; 11:787-97.

40. Overman MJ, Maru DM, Charnsangavej C, et al. Oxaliplatin-mediated increase in spleen size as a biomarker for the development of hepatic sinusoidal injury. J Clin Oncol 2010; 28:2549-55.

41. Eriksson S, Fraser JR, Laurent TC, et al. Endothelial cells are a site of uptake and degradation of hyaluronic acid in the liver. Exp Cell Res 1983; 144:223-8. 
42. Fraser JR, Laurent TC, Laurent UB. Hyaluronan: its nature, distribution, functions and turnover. J Intern Med 1997; 242:27-33.

43. Tamandl D, Klinger M, Eipeldauer S, et al. Sinusoidal obstruction syndrome impairs long-term outcome of colorectal liver metastases treated with resection after neoadjuvant chemotherapy. Ann Surg Oncol 2010; 18:421-30.

44. Rubbia-Brandt L, Giostra E, Brezault C, et al. Importance of histological tumor response assessment in predicting the outcome in patients with colorectal liver metastases treated with neoadjuvant chemotherapy followed by liver surgery. Ann Oncol 2007; 18:299-304.

45. Mandard AM, Dalibard F, Mandard JC, et al. Pathologic assessment of tumor regression after preoperative chemoradiotherapy of esophageal carcinoma. Clinicopathologic correlations. Cancer 1994; 73:2680-6.

46. DeLeve LD, McCuskey RS, Wang X, et al. Characterization of a reproducible rat model of hepatic veno-occlusive disease. Hepatology 1999; 29:1779-91.

47. Bast A, Haenen GR, Bruynzeel AM, Van der Vijgh WJ. Protection by flavonoids against anthracycline cardiotoxicity: from chemistry to clinical trials. Cardiovasc Toxicol 2007; 7:154-9.

48. Wadworth AN, Faulds D. Hydroxyethylrutosides. A review of its pharmacology, and therapeutic efficacy in venous insufficiency and related disorders. Drugs 1992; 44:1013-32. 
Chapter 13

Clinical implications 



\section{PART I. ASSESSING OUTCOMES OF LIVER SURGERY: CRITICAL APPRAISAL OF CLINICAL ENDPOINTS}

\section{Observation:}

In current HPB practice, liver surgery-related mortality and morbidity rates are low: operative mortality has a mean rate of 1 per cent and the mean rates of specific types of morbidity are all below 8 per cent. Consequently, the sample size required for an adequately powered randomized controlled trial (RCT) in liver surgery using surgeryrelated mortality or morbidity as primary endpoint turns out to be extremely large.

\section{Implication:}

The feasibility of RCTs in liver surgery using clinical outcomes as primary endpoint is low as such endpoints require large sample sizes owing to their low incidence. Strategies that may enable the conduct of high level of evidence research in liver surgery using clinical outcomes include the use of composite endpoints, organization of multicentre trials and performance of meta-analyses.

\section{Observation:}

Clear definitions of clinical outcomes, used as primary or secondary endpoints in RCTs in liver surgery, are provided in less than one-third of trial reports. If provided, the definitions differ substantially between studies.

\section{Implication:}

The lack of uniform definitions hampers the interpretation of trial results and compromises the validity of systematic reviews and meta-analyses in HPB surgery. Our attempt to design consensus-based standard definitions of clinical outcomes of liver surgery may solve this problem. Prospective validation of the proposed definitions is warranted.

\section{Observation:}

The use of a composite endpoint (CEP) as primary endpoint of RCTs in liver surgery is not very common at present and uniformity of CEP components is lacking. A well-defined liver surgery specific CEP, consisting of ascites, post-resectional liver failure, bile leakage, intra-abdominal abscess, intra-abdominal hemorrhage and operative mortality is proposed. This liver surgery specific CEP has a considerably higher event rate than any of its individual components. 


\section{Implication:}

Implementation of a liver surgery specific CEP potentially increases the feasibility of conducting RCTs using clinical outcomes of liver surgery as primary endpoint. Its significantly higher incidence rate will decrease the number of patients needed for an adequately powered trial. It is important to keep in mind that the use of CEPs can be misleading if components vary widely in clinical relevance or incidence. Therefore, components should be classified using an objective severity grading system and the incidence of all components should be reported. Prospective validation of the proposed liver surgery specific CEP is warranted.

\section{PART II. ASSESSING OUTCOMES OF LIVER SURGERY: HEPATIC DAMAGE AS A RESULT OF SURGICAL TECHNIQUE}

\section{Observation:}

Liver mobilization during liver surgery in man is associated with hepatocellular damage and liver inflammation. The amount of liver inflammation is significantly associated with the duration of inflammation.

\section{Implication:}

As mobilization-induced hepatocellular damage might have a negative impact on shortterm outcomes of liver surgery, preventive strategies may be beneficial. They include either employment of alternative surgical techniques, such as the anterior approach or laparoscopic surgery, or modulation of inflammation by anti-inflammatory drugs.

\section{Observation:}

An intermittent Pringle maneuver using $30 \mathrm{~min}$ ischemic intervals induces a similar amount of hepatocellular damage, reflected by systemic liver fatty acid-binding protein levels, compared to $15 \mathrm{~min}$ ischemic intervals, without loss of remnant liver function. No Pringle maneuver leads to significantly less liver cell damage.

\section{Implication:}

It is preferable not to use inflow occlusion during liver resection. If inflow occlusion is required, 30 minutes ischemic intervals may be used. 


\section{PART III. ASSESSING OUTCOMES OF LIVER SURGERY: HEPATIC DAMAGE AS A RESULT OF OXALIPLATIN-BASED CHEMOTHERAPY}

\section{Observation:}

Oxaliplatin-based neoadjuvant chemotherapy causes sinusoidal injury of the nontumour-bearing liver consistent with nodular regenerative hyperplasia. This condition is associated with the development of portal hypertension.

\section{Implication:}

In patients with colorectal liver metastases (CLMs), the indication for neoadjuvant chemotherapy treatment should be evaluated carefully by a multidisciplinary team. The benefit of tumour downsizing should be weighed against the disadvantage of induction of sinusoidal injury. If deemed necessary, caution should be exercised in patients treated with oxaliplatin-based chemotherapy prior to major liver surgery. Thorough analysis of liver volume and function should guide the operative strategy. When future remnant liver volume is regarded insufficient, portal vein embolization, two stage hepatectomy, ALPPS approach or restrictive surgery should be considered.

\section{Observation:}

Elevated systemic hyaluronic acid levels have a fair diagnostic performance for the non-invasive detection of patients with sinusoidal obstruction syndrome (SOS) after oxaliplatin-based chemotherapy for CLMs. The net functional clearance capacity of hepatic sinusoidal endothelial cells (SECs) for hyaluronic acid seems to be preserved in patients with SOS.

\section{Implication:}

High systemic hyaluronic acid levels identify patients at risk of SOS. In these patients, additional investigations into the presence of SOS are indicated. The presence of sinusoidal injury per se does not seem to interfere with the capacity of hepatic SECs to clear hyaluronic acid. From a mechanistic point of view, it would be relevant to obtain a more detailed insight into hyaluronic acid metabolism and SEC function in patients with SOS secondary to oxaliplatin-based chemotherapy by either performing hyaluronic acid loading tests or PET scans with $\left[{ }^{11} \mathrm{C}\right]$ hyaluronic acid. 


\section{Observation:}

The incidence of SOS of the non-tumour-bearing liver in patients with CLMs treated with oxaliplatin-based chemotherapy prior to surgery is approximately 60 per cent. The tumour regression grade is increased in patients suffering from SOS.

\section{Implication:}

An increased tumour regression grade is consistent with a decreased tumour response to chemotherapy. Tumour regression grade and tumour response are important for the prediction of long-term survival and consequently, patients with SOS who exhibit a decreased tumour response may have an unfavorable long-term clinical outcome. Therefore, it seems to be clinically relevant to develop strategies that prevent SOS secondary to oxaliplatin-based chemotherapy.

\section{Observation:}

MonoHER, a flavonoid with antioxidant, anti-inflammatory and vaso-active properties, is able to prevent the development of portal hypertension, hepatocellular damage and hepatic sinusoidal endothelial cell injury in an experimental rat model of SOS. Moreover, there is no effect of monoHER on the cytotoxic capacity of oxaliplatin in vitro in different colorectal cancer cell lines.

\section{Implication:}

Administration of monoHER is successful in preventing SOS in an experimental rat model. Phase I and II clinical trials with monoHER have been performed in man without evidence for serious side effects of monoHER administration. Translation of our experimental observations to man is warranted as validated strategies to prevent sinusoidal injury secondary to oxaliplatin-based chemotherapy for CLMs in man are lacking to date. 
Chapter 14

Future perspectives 

The broader aim of the present thesis was to assess and improve outcomes of liver surgery. The studies described in this thesis focused on the conduct of high level of evidence research in liver surgery as well as surgery and patient-related risk factors of postresectional liver failure (PLF). Based on the results of the present thesis, several clinical questions have been answered. However, probably an even larger number of questions have been generated that warrant future investigation. Some interesting, unresolved issues with high clinical relevance will be discussed shortly in the next paragraphs.

\section{ARE WE USING THE RIGHT OUTCOMES AS ENDPOINTS OF RANDOMIZED CONTROLLED TRIALS IN LIVER SURGERY?}

The conduct of appropriately powered randomized controlled trials (RCTs) in liver surgery using clinical outcomes as primary endpoints is hardly feasible due to declining event rates (chapter 2). In order to overcome this problem, composite or surrogate endpoints may be used as primary endpoints. Whereas the pros and cons of the use of composite endpoints (CEPs) have been extensively discussed in the present thesis (chapter 4), the use of surrogate endpoints (SEPs) has not. The US Food and Drug Administration defined SEPs as "laboratory measurements or physical signs that are used in therapeutic trials as substitutes for true, clinically meaningful endpoints that are direct measures of how a patient feels, functions or survives, and are expected to predict the effect of therapy". The use of SEPs is attractive as they potentially reduce the required sample size, costs and inclusion periods of RCTs, which allows more rapid testing and implementation of treatment strategies ${ }^{1-3}$.

SEPs that are frequently used in liver surgery-related trials include hepatocellular damage markers such as aminotransferases as surrogates for liver surgery-related morbidity, hepatic function markers such as bilirubin level or prothrombin index as surrogates for PLF, or progression-free survival as surrogate for overall survival (see ${ }^{4-6}$ ). Unfortunately, robust validation of these commonly used SEPs in liver surgery is lacking to date. In 1989, Prentice described four criteria for the identification of valid SEPs. These criteria can be summarized as follows: (1) the intervention has a statistically significant impact on the SEP, (2) the intervention has a statistically significant impact on the true, clinical endpoint, (3) the SEP has a statistically significant impact on the true, clinical endpoint, and (4) the full effect of the treatment on the true, clinical endpoint must be captured by the $\mathrm{SEP}^{1,3}$. In several fields in medicine, critical appraisal of frequently used surrogates has been performed using these Prentice criteria ${ }^{3,7}$. In liver surgery, such a formal validation of the commonly used SEPs is urgently warranted. This could be achieved by statistical analysis of combined data from multiple large RCTs in liver surgery that recorded both the occurrence of the surrogate and clinical endpoint. Up 
until formal validation, the effect of the intervention on the true, clinical endpoint in liver surgery-related trials using a SEP as primary outcome parameter should be cautiously considered.

\section{IS MODULATION OF INFLAMMATION BENEFICIAL IN PREVENTING MOBILIZATION-INDUCED HEPATOCELLULAR DAMAGE?}

We showed that manipulation of the liver during liver surgery in humans was accompanied by hepatocellular damage, increased influx of inflammatory cells, upregulation of cellular adhesion molecules and enhanced expression of pro-inflammatory cytokines (chapter 5). Experimental studies have delivered convincing evidence that inflammation was causally involved in the process of mobilization-induced hepatocellular damage ${ }^{8,9}$. When the inflammatory response was blunted by either depletion of Kupffer cells or modulation of Kupffer cell activity, liver cell damage decreased and clinical outcomes improved ${ }^{9,10}$. From this, we speculate that modulation of inflammation by administration of agents that inhibit the activation or influx of cells that respond first to tissue injury, i.e. macrophages, neutrophils and mast cells, would also be beneficial in man ${ }^{11}$. Potential drugs that dampen inflammation include neutralizers of endogenous cytokines (e.g. anti-tumour necrosis factor-a), inhibitors of inflammatory cell influx (e.g. intracellular adhesion molecule antibodies), and stabilizers of mast cells or antagonists of mast cell releasate (e.g. antihistamines) ${ }^{12,13}$. With respect to the latter, several lines of evidence in the field of manipulation-induced intestinal injury suggest that mast cells are involved in the attraction of inflammatory cells to the site of manipulation, whereas their depletion or inhibition of degranulation was effective in preventing intestinal inflammation in rodents and $\operatorname{man}^{13,14}$. As mast cells are abundantly present in the liver ${ }^{15}$, inhibition of their degranulation or blocking of certain histamine receptors during manipulation of the liver may be beneficial in preventing manipulation-induced hepatocellular damage and inflammation. We propose to study the role of mast cells and effect of mast cell stabilizers in an experimental model of liver manipulation. If proven effective, these results could be translated to patients undergoing surgery requiring manipulation of the liver in a randomized controlled fashion. 


\section{WHICH MEASURES ARE ABLE TO PREVENT SINUSOIDAL INJURY SECONDARY TO OXALIPLATIN-BASED CHEMOTHERAPY IN PATIENTS WITH COLORECTAL LIVER METASTASES?}

Oxaliplatin-based chemotherapy is a pivotal element in the multimodal approach to patients with colorectal liver metastases (CLMs). One of the side-effects of oxaliplatin is injury to hepatic sinusoidal endothelial cells (SECs), characterized as sinusoidal obstruction syndrome (SOS). The presence of SOS is related to enhanced post-resectional morbidity after major liver surgery (chapter 7$)^{16,17}$. Endothelial cell damage, inflammation and oxidative stress all are involved in the pathophysiology of $\mathrm{SOS}^{18}$. Intravenous or intraperitoneal administration of anti-oxidants, vasoprotectors, nitric oxide-analogues, or matrix metalloproteinase inhibitors proved to be effective in preventing SOS in an experimental rat model ${ }^{19-22}$. We showed that intraperitoneal injection of monoHER was able to prevent SOS in rats (chapter 10). Moreover, we found no effect of monoHER on the cytotoxicity of oxaliplatin in vitro in colon cancer cell lines. However, evidence of the efficacy of aforementioned agents in preventing SOS in man is lacking to date.

To confirm our results on the effectiveness of monoHER in preventing SOS in man, we propose to conduct a clinical phase II study in patients with CLMs treated with oxaliplatinbased neoadjuvant chemotherapy. A clinical phase I study on the safety of the effective intravenous monoHER dose in healthy volunteers has already been performed ${ }^{23}$. The proposed phase II study should aim at (1) determination of the optimal dose and timing of monoHER administration, (2) assessment of the protective effect of monoHER on SOS by analysis of circulating endothelial damage markers and histological evidence of sinusoidal injury, (3) evaluation of the effect of monoHER on objective tumour response, and (4) analysis of the effect of monoHER on the preservation of remnant liver function ${ }^{24}$.

In addition, other promising agents to protect the liver from sinusoidal injury secondary to oxaliplatin-based chemotherapy exist, such as intravenous administration of bevacizumab or oral aspirin treatment ${ }^{25-27}$. With respect to the latter, aspirin has been shown to prevent SOS after oxaliplatin-based chemotherapy in two independent, non-randomized studies in humans ${ }^{27}, 28$. The mechanism underlying the protective effect of aspirin is unknown and may include inhibition of platelet aggregation as well as modulation of inflammation ${ }^{29}$. As the administration of aspirin is simple, safe, and inexpensive, an RCT analyzing the effect of oral aspirin treatment on sinusoidal injury and post-resectional outcomes in patients with CLMs receiving oxaliplatin-based chemotherapy seems to be a next, logical step. 


\section{REFERENCES}

1. Prentice RL. Surrogate endpoints in clinical trials: definition and operational criteria. Stat Med 1989; 8: 431-40.

2. Boissel JP, Collet JP, Moleur P, Haugh M. Surrogate endpoints: a basis for a rational approach. Eur J Clin Pharmacol 1992; 43: 235-44.

3. Ray ME, Bae K, Hussain MH, Hanks GE, Shipley WU, Sandler HM. Potential surrogate endpoints for prostate cancer survival: analysis of a phase III randomized trial. J Natl Cancer Inst 2009; 101: 228-36.

4. Beck-Schimmer B, Breitenstein S, Urech S, De Conno E, Wittlinger M, Puhan M, et al. A randomized controlled trial on pharmacological preconditioning in liver surgery using a volatile anesthetic. Ann Surg 2008; 248: 909-18.

5. Esaki M, Sano T, Shimada K, Sakamoto Y, Takahashi Y, Wakai K, et al. Randomized clinical trial of hepatectomy using intermittent pedicle occlusion with ischaemic intervals of 15 versus 30 minutes. Br J Surg 2006; 93: 944-51.

6. Nordlinger B, Sorbye H, Glimelius B, Poston GJ, Schlag PM, Rougier P, et al. Perioperative chemotherapy with FOLFOX4 and surgery versus surgery alone for resectable liver metastases from colorectal cancer (EORTC Intergroup trial 40983): a randomised controlled trial. Lancet 2008; 371 : 1007-16.

7. Schold JD, Kaplan B. The elephant in the room: failings of current clinical endpoints in kidney transplantation. Am J Transplant 2010; 10: 1163-6.

8. Schemmer P, Schoonhoven R, Swenberg JA, Bunzendahl H, Thurman RG. Gentle in situ liver manipulation during organ harvest decreases survival after rat liver transplantation: role of Kupffer cells. Transplantation 1998; 65: 1015-20.

9. Schemmer P, Enomoto N, Bradford BU, Bunzendahl H, Raleigh JA, Thurman RG. Autonomic nervous system and gut-derived endotoxin: involvement in activation of Kupffer cells after in situ organ manipulation. World J Surg 2001; 25: 399-406.

10. Schemmer P, Connor HD, Arteel GE, Raleigh JA, Bunzendahl H, Mason RP, et al. Reperfusion injury in livers due to gentle in situ organ manipulation during harvest involves hypoxia and free radicals. J Pharmacol Exp Ther 1999; 290: 235-40.

11. Nathan C. Points of control in inflammation. Nature 2002; 420: 846-52.

12. The FO, de Jonge WJ, Bennink RJ, van den Wijngaard RM, Boeckxstaens GE. The ICAM-1 antisense oligonucleotide ISIS-3082 prevents the development of postoperative ileus in mice. Br J Pharmacol 2005; 146: 252-8.

13. The FO, Buist MR, Lei A, Bennink RJ, Hofland J, van den Wijngaard RM, et al. The role of mast cell stabilization in treatment of postoperative ileus: a pilot study. Am J Gastroenterol 2009; 104 : 2257-66.

14. de Jonge WJ, The FO, van der Coelen D, Bennink RJ, Reitsma PH, van Deventer SJ, et al. Mast cell degranulation during abdominal surgery initiates postoperative ileus in mice. Gastroenterology 2004; 127: 535-45.

15. Francis $\mathrm{H}$, Meininger $\mathrm{CJ}$. A review of mast cells and liver disease: What have we learned? Dig Liver Dis 2010; 42: 529-36.

16. Soubrane O, Brouquet A, Zalinski S, Terris B, Brezault C, Mallet V, et al. Predicting high grade lesions of sinusoidal obstruction syndrome related to oxaliplatin-based chemotherapy for colorectal liver metastases: correlation with post-hepatectomy outcome. Ann Surg 2010; 251: 454-60. 
17. Karoui M, Penna C, Amin-Hashem M, Mitry E, Benoist S, Franc B, et al. Influence of preoperative chemotherapy on the risk of major hepatectomy for colorectal liver metastases. Ann Surg 2006; 243: 1-7.

18. DeLeve LD. Hepatic microvasculature in liver injury. Semin Liver Dis 2007; 27: 390-400.

19. Deleve LD, Wang X, Tsai J, Kanel G, Strasberg S, Tokes ZA. Sinusoidal obstruction syndrome (venoocclusive disease) in the rat is prevented by matrix metalloproteinase inhibition. Gastroenterology 2003; 125: 882-90.

20. DeLeve LD, Wang X, Kanel GC, Ito Y, Bethea NW, McCuskey MK, et al. Decreased hepatic nitric oxide production contributes to the development of rat sinusoidal obstruction syndrome. Hepatology 2003; 38: 900-8.

21. Narita M, Hatano E, Tamaki N, Yamanaka K, Yanagida A, Nagata H, et al. Dai-kenchu-to attenuates rat sinusoidal obstruction syndrome by inhibiting the accumulation of neutrophils in the liver. $J$ Gastroenterol Hepatol 2009; 24: 1051-7.

22. Narita M, Hatano E, Ikai I, Miyagawa-Hayashino A, Yanagida A, Nagata H, et al. A phosphodiesterase III inhibitor protects rat liver from sinusoidal obstruction syndrome through heme oxygenase-1 induction. Ann Surg 2009; 249: 806-13.

23. Willems AM, Bruynzeel AM, Kedde MA, van Groeningen CJ, Bast A, van der Vijgh WJ. A phase I study of monohydroxyethylrutoside in healthy volunteers. Cancer Chemother Pharmacol 2006; 57 : 678-84.

24. Bruynzeel AM, Niessen HW, Bronzwaer JG, van der Hoeven JJ, Berkhof J, Bast A, et al. The effect of monohydroxyethylrutoside on doxorubicin-induced cardiotoxicity in patients treated for metastatic cancer in a phase II study. Br J Cancer 2007; 97: 1084-9.

25. Klinger M, Eipeldauer S, Hacker S, Herberger B, Tamandl D, Dorfmeister M, et al. Bevacizumab protects against sinusoidal obstruction syndrome and does not increase response rate in neoadjuvant XELOX/FOLFOX therapy of colorectal cancer liver metastases. Eur J Surg Oncol 2009; 35 : 515-20.

26. Rubbia-Brandt L, Lauwers GY, Wang H, Majno PE, Tanabe K, Zhu AX, et al. Sinusoidal obstruction syndrome and nodular regenerative hyperplasia are frequent oxaliplatin-associated liver lesions and partially prevented by bevacizumab in patients with hepatic colorectal metastasis. Histopathology 2010; 56: 430-9.

27. Brouquet A, Benoist S, Julie C, Penna C, Beauchet A, Rougier P, et al. Risk factors for chemotherapy-associated liver injuries: A multivariate analysis of a group of 146 patients with colorectal metastases. Surgery 2009; 145: 362-71.

28. Agostini J, Benoist S, Seman M, Julie C, Imbeaud S, Letourneur F, et al. Identification of molecular pathways involved in oxaliplatin-associated sinusoidal dilatation. J Hepatol 2012; 56: 869-76.

29. Dinarello CA. Anti-inflammatory Agents: Present and Future. Cell 2010; 140: 935-50. 

Appendices 

APPENDIX 1. CHECKLIST FOR THE SELECTION OF ARTICLES FOR FINAL

ANALYSIS.

\section{Details of article}

Title

First author

Institute

Year of publication

\begin{tabular}{l}
\hline Prerequisites for inclusion $\quad$ yes \\
*More than 50 patients \\
${ }^{*}$ More than 15 patients/year \\
${ }^{*}$ All patient categories \\
${ }^{*}$ All hepatectomies \\
*Published between 2002-2007 \\
${ }^{*}$ No review or case report \\
No RCT with exclusion criteria
\end{tabular}

$\mathrm{RCT}$, randomized controlled trial; * item used for article selection.

\begin{tabular}{|c|c|c|c|}
\hline Article review & & & Quality score \\
\hline \multirow[t]{3}{*}{ *Study design } & $\mathrm{RCT}$ & & 5 \\
\hline & cohort study & prospective & 3 \\
\hline & & retrospective & 1 \\
\hline *Inclusion period & & & 1 \\
\hline${ }^{*}$ Number of patients & & & 1 \\
\hline \multicolumn{4}{|l|}{$\begin{array}{l}{ }^{*} \text { Number of } \\
\text { hepatectomies }\end{array}$} \\
\hline \multicolumn{4}{|l|}{${ }^{*}$ Exclusion criteria } \\
\hline *Mortality (\%) & & & 1 \\
\hline \multirow[t]{3}{*}{${ }^{*}$ Mortality index used } & 30-day & in-hospital & 1 \\
\hline & operative & not specified & \\
\hline & total & & \\
\hline Cause of death specified & & & 1 \\
\hline *Morbidity (\%) & major & minor & 1 \\
\hline \multirow[t]{3}{*}{ Morbidity index used } & 30-day & in-hospital & 1 \\
\hline & operative & not specified & \\
\hline & total & & \\
\hline Outpatient data included & & & 1 \\
\hline \multirow{2}{*}{$\begin{array}{l}\text { *Procedure specific } \\
\text { morbidity }\end{array}$} & *PLF & & 1 \\
\hline & *bile leakage & & 1 \\
\hline
\end{tabular}




\begin{tabular}{ll}
\hline Article review & Quality score \\
\hline *Definitions provided & 1 \\
Severity grading & 1 \\
Length of stay data & 1 \\
Risk factors analyzed & 1 \\
\hline
\end{tabular}

\section{Total score}

$\mathrm{RCT}$, randomized controlled trial; PLF, post-resectional liver failure; * item used for article selection.

\section{Article inclusion for final analysis}

YES

\section{Details of articles selected for analysis}

Article reference check

$\begin{array}{lll}\text { Age } & \text { mean } \pm \text { standard deviation } & \text { median (range) } \\ \text { Sex } & \text { female } & \text { male } \\ \text { Indication for resection } & \text { primary liver tumour } & \text { transplantation } \\ & \text { secondary liver tumour } & \text { other } \\ & \text { benign disease } & \text { minor } \\ \text { Type of resection } & \text { major } & \text { median (range) } \\ \text { Duration of operation } & \text { mean } \pm \text { standard deviation } & \text { median (range) } \\ \text { Clamping time } & \text { mean } \pm \text { standard deviation } & \text { median (range) } \\ \text { Blood loss } & \text { mean } \pm \text { standard deviation } & \text { median (range) } \\ \text { Hospital stay } & \text { mean } \pm \text { standard deviation } & \end{array}$

\section{MAYBE}

NO MAYBE




\section{APPENDIX 2. FORMULA USED FOR SAMPLE SIZE CALCULATION OF EACH} TREATMENT ARM OF A RANDOMIZED CONTROLLED TRIAL'.

$$
N_{1}=N_{2}=\left(c z_{1-\beta}+z_{1-\alpha / 2}\right)^{2} \times \frac{2 \sigma^{2}}{\left(\pi_{1}-\Pi_{2}\right)^{2}}
$$

in which:

$N=$ number of patients needed per treatment arm

$\pi_{1}=$ incidence in control condition

$\pi_{2}=$ incidence in intervention condition

$\sigma^{2}=$ pooled variance $=\pi(1-\pi)$, with $\pi=\left(\pi_{1}+\pi_{2}\right) / 2$

$C=\sqrt{ }\left\{\left[\pi_{1}\left(1-\pi_{1}\right)+\pi_{2}\left(1-\pi_{2}\right)\right] / 2 \sigma^{2}\right\}^{*}$

$\mathrm{z}_{1-\beta}=$ B-term

$z_{1-\alpha / 2}=$ A-term

${ }^{*} \mathrm{c}<1$ always and $\mathrm{c} \approx 1$ usually, so $\mathrm{c}=1$ is an acceptable simplification 


\section{APPENDIX 3. OVERVIEW OF 19 ARTICLES WITH OVERLAPPING DATA AND THEIR METHODOLOGICAL QUALITY SCORE.}

\begin{tabular}{llllcc}
\hline No & Author & Year & Journal & N patients & Quality score \\
\hline 1 & Arru * & 2007 & Am Surg & 100 & 11 \\
2 & Aldrighetti & 2006 & J Gastrointest Surg & 200 & 9 \\
\hline 3 & Benzoni $^{*}$ & 2007 & Lagenbecks Arch Surg & 287 & 9 \\
4 & Benzoni & 2006 & Hepatobiliary Pancreat Dis Int & 287 & 9 \\
\hline 5 & Capussotti * & 2006 & Arch Surg & 610 & 11 \\
6 & Capussotti & 2006 & Br J Surg & 126 & 11 \\
\hline 7 & Sun * & 2006 & Br J Surg & 120 & 14 \\
8 & Sun & 2005 & Hepatobiliary Pancreat Dis Int & 146 & 11 \\
\hline 9 & Aldameh * & 2005 & J Gastrointest Surg & 211 & 11 \\
10 & Koea & 2005 & New Zealand Medical J & 100 & 14 \\
\hline 11 & Imamura * & 2003 & Arch Surg & 915 & 11 \\
12 & Hasegawa & 2002 & Arch Surg & 80 & 14 \\
\hline 13 & Aldrighetti * & 2003 & World J Surg & 129 & 11 \\
14 & Aldrighetti & 2004 & Am Surg & 103 & 10 \\
\hline 15 & Jarnagin * & 2002 & Ann Surg & 1803 & 12 \\
16 & Burt & 2002 & Am J Surg & 1165 & 10 \\
\hline 17 & D'Angelica & 2006 & World J Surg & 1426 & 9 \\
\hline 19 & Kimura * & 2006 & J Surg Res & 128 & 12 \\
\hline
\end{tabular}

$N$, number; For details on quality scoring see Appendix 1 ; ${ }^{*}$ selected article.

The selected articles (marked with *) had a higher quality score. When articles had the same score, either the largest or latest publication was chosen. Exceptions were:

- The article by Koea and colleagues was not selected because it had a smaller patient number.

- The article by Imamura and colleagues was selected because it described a considerably larger patient number. 
APPENDIX 4. REFERENCE LIST OF ARTICLES INCLUDED IN THE FINAL

ANALYSIS.

\begin{tabular}{|c|c|c|c|c|c|c|c|c|}
\hline \multirow[t]{2}{*}{ No } & \multirow[t]{2}{*}{ Author } & \multirow{2}{*}{$\begin{array}{c}\text { Quality } \\
\text { score }\end{array}$} & \multirow{2}{*}{$\begin{array}{c}N \\
\text { patients }\end{array}$} & \multicolumn{5}{|c|}{ Complication rate (\%) } \\
\hline & & & & PLF & $\begin{array}{l}\text { Intra- } \\
\text { abdominal } \\
\text { abscess }\end{array}$ & $\begin{array}{c}\text { Bile } \\
\text { leakage }\end{array}$ & $\begin{array}{c}\text { Intra- } \\
\text { abdominal } \\
\text { hemorrhage }\end{array}$ & Mortality* \\
\hline 1 & Hutchins & 7 & 89 & - & 1.1 & 5.6 & - & 1.1 (op) \\
\hline 2 & Ayav & 12 & 236 & 0.8 & 8.5 & 2.1 & - & 2.1 (op) \\
\hline 3 & Milićević & 11 & 90 & 1.1 & 4.4 & - & - & 3.3 (op) \\
\hline 4 & Watanabe & 12 & 151 & 18.5 & 13.2 & 11.9 & 3.3 & 6.6 (op) \\
\hline 5 & Figueras & 15 & 300 & 6.0 & 1.0 & 10.7 & - & 2.7 (ho) \\
\hline 6 & Arru & 11 & 100 & 2.0 & - & 2.0 & 1.0 & 1.0 (op) \\
\hline 7 & Pessaux & 17 & 200 & 10.0 & 6.0 & 8.5 & 2.0 & $2.5(30)$ \\
\hline 8 & Herman & 10 & 278 & 0.7 & 3.2 & 5.8 & 0.7 & 1.1 (op) \\
\hline 9 & Evrard & 12 & 80 & 1.3 & 1.3 & 5.0 & 3.8 & 1.3 (op) \\
\hline 10 & Benzoni & 9 & 287 & 9.1 & 11.8 & 2.8 & 4.9 & 4.5 (ho) \\
\hline 11 & Kwon & 11 & 178 & 1.1 & 5.6 & 8.4 & 3.4 & 1.1 (op) \\
\hline 12 & Capussotti & 11 & 610 & - & - & 3.6 & - & 2.5 (ho) \\
\hline 13 & Saiura & 11 & 60 & - & - & 6.7 & 1.7 & 0.0 (ho) \\
\hline 14 & Sawada & 9 & 91 & - & - & 4.4 & - & 0.0 (op) \\
\hline 15 & Sun & 14 & 120 & 1.7 & 3.3 & 0.0 & 0.8 & 0.8 (ho) \\
\hline 16 & $\mathrm{Lu}$ & 12 & 462 & 1.7 & 1.7 & 1.3 & 0.9 & 0.4 (ho) \\
\hline 17 & $\mathrm{Wu}$ & 14 & 214 & - & 6.5 & 4.7 & - & 0.0 (ho) \\
\hline 18 & Kim & 15 & 60 & 1.7 & 3.3 & 1.7 & 6.7 & 0.0 (op) \\
\hline 19 & Torzilli & 13 & 58 & - & - & 0.0 & - & $0.0(30)$ \\
\hline 20 & Aldameh & 11 & 211 & 3.8 & 1.9 & 3.3 & - & 1.4 (op) \\
\hline 21 & Schindl & 9 & 104 & 12.5 & - & 5.8 & 1.9 & 1.9 (op) \\
\hline 22 & Gruttadauria & 9 & 149 & 2.7 & 3.4 & 10.1 & - & 3.4 (op) \\
\hline 23 & Poon & 11 & 1222 & 3.8 & 2.7 & 3.1 & 1.6 & 4.9 (ho) \\
\hline 24 & Imamura & 11 & 1056 & 0.1 & 8.2 & 9.2 & 0.9 & 0.0 (op) \\
\hline 25 & Aldrighetti & 11 & 129 & 3.9 & - & 3.9 & 0.8 & $0.8(30)$ \\
\hline 26 & Yanaga & 11 & 60 & 1.7 & 8.3 & 6.7 & - & 0.0 (ho) \\
\hline 27 & Bober & 9 & 99 & 1.0 & 3.0 & 2.0 & 5.1 & 0.0 (op) \\
\hline 28 & Muratore & 13 & 53 & 1.9 & 13.2 & - & 1.9 & 0.0 (op) \\
\hline 29 & Jarnagin & 12 & 1803 & 5.5 & 6.1 & 2.6 & 1.0 & 3.1 (op) \\
\hline 30 & Kim & 13 & 66 & 3.0 & 4.5 & 4.5 & 4.5 & 1.5 (ho) \\
\hline 31 & Arita & 16 & 80 & - & 2.5 & 11.3 & - & 0.0 (op) \\
\hline 32 & Kimura & 12 & 128 & 4.7 & 21.1 & - & 3.1 & 1.6 (op) \\
\hline
\end{tabular}

$N$, number; PLF, post-resectional liver failure; -, not reported; ${ }^{*}$ Mortality index: (30) for 30-day mortality, (ho) for in-hospital mortality, and (op) for operative mortality. 
APPENDIX 5. REFERENCE LIST OF RANDOMIZED CONTROLLED TRIALS INCLUDED IN THE FINAL ANALYSIS.

\begin{tabular}{|c|c|c|c|c|c|c|}
\hline No & Author & $N$ patients & $\begin{array}{c}\text { Multicentre } \\
\text { trial }\end{array}$ & $\begin{array}{l}\text { Primary } \\
\text { endpoint }\end{array}$ & $\begin{array}{l}\text { Composite } \\
\text { endpoint }\end{array}$ & $\begin{array}{l}\text { Overlapping } \\
\text { data* }\end{array}$ \\
\hline 1 & Beck-Schimmer & 64 & No & Continuous & No & \\
\hline 2 & Jarnagin & 130 & No & Dichotomous & No & \\
\hline 3 & Torzilli & 76 & No & Continuous & No & \\
\hline 4 & Ko & 70 & No & Continuous & No & \\
\hline 5 & Heizmann & 61 & No & Dichotomous & Yes & \\
\hline 6 & Nordlinger & 364 & Yes & Continuous & No & \\
\hline 7 & Yuksel & 32 & No & Dichotomous & Yes & \\
\hline 8 & Kim & 130 & No & Continuous & No & \\
\hline 9 & Pulitano & 43 & No & Continuous & No & $A$ \\
\hline 10 & Schmidt & 20 & No & Continuous & No & \\
\hline 11 & Pulitano & 73 & No & Continuous & No & A \\
\hline 12 & Campagnacci & 24 & No & Continuous & No & \\
\hline 13 & Hashimoto & 79 & No & Continuous & No & \\
\hline 14 & Kostopanagiotou & 35 & No & Continuous & No & B \\
\hline 15 & Figueras & 300 & No & Dichotomous & Yes & \\
\hline 16 & Togo & 180 & No & Dichotomous & Yes & \\
\hline 17 & Lupo & 50 & No & Dichotomous & Yes & \\
\hline 18 & Pessaux & 200 & No & Dichotomous & Yes & \\
\hline 19 & Shi & 169 & No & Dichotomous & No & C \\
\hline 20 & Smyrniotis & 54 & No & Continuous & No & B \\
\hline 21 & Petrowsky & 73 & No & Continuous & No & \\
\hline 22 & Sugawara & 81 & No & Continuous & No & \\
\hline 23 & Liu & 120 & No & Dichotomous & No & $\mathrm{D}$ \\
\hline 24 & Esaki & 92 & No & Continuous & No & \\
\hline 25 & Saiura & 60 & No & Continuous & No & \\
\hline 26 & Aldrighetti & 76 & No & Continuous & No & A \\
\hline 27 & Capussotti & 126 & No & Continuous & No & \\
\hline 28 & Chen & 118 & No & Continuous & No & \\
\hline 29 & Wang & 50 & No & Continuous & No & C \\
\hline 30 & Sun & 120 & No & Dichotomous & No & \\
\hline 31 & Shao & 231 & Yes & Dichotomous & No & \\
\hline 32 & Wu & 214 & No & Dichotomous & No & \\
\hline 33 & Azoulay & 60 & No & Continuous & No & \\
\hline 34 & Kim & 60 & No & Continuous & No & \\
\hline 35 & Yoshida & 49 & No & Dichotomous & No & \\
\hline 36 & Arita & 80 & No & Continuous & No & \\
\hline
\end{tabular}




\begin{tabular}{llcclll}
\hline No & Author & N patients & $\begin{array}{c}\text { Multicentre } \\
\text { trial }\end{array}$ & $\begin{array}{l}\text { Primary } \\
\text { endpoint }\end{array}$ & $\begin{array}{l}\text { Composite } \\
\text { endpoint }\end{array}$ & $\begin{array}{l}\text { Overlapping } \\
\text { data* }\end{array}$ \\
\hline 37 & Figueras & 80 & No & Continuous & No & \\
38 & Smyrniotis & 82 & No & Dichotomous & Yes & B \\
39 & Kanazawa & 44 & No & Continuous & No & \\
40 & Lodge & 185 & Yes & Dichotomous & No & \\
41 & Frilling & 121 & Yes & Continuous & No & \\
42 & Lesurtel & 100 & No & Continuous & No & \\
43 & Schwartz & 121 & Yes & Continuous & No & \\
44 & Li & 29 & No & Continuous & No & C \\
45 & Fuster & 40 & No & Dichotomous No & \\
46 & Nuzzo & 42 & No & Dichotomous & Yes & \\
47 & Liu & 104 & No & Dichotomous & No & D \\
\hline
\end{tabular}

$\mathrm{N}$, number; * Capital letters refer to randomized controlled trials published by one institute using data from overlapping time periods. 


\section{APPENDIX 6. OVERVIEW OF DEFINITIONS OF CLINICAL OUTCOMES USED AS ENDPOINTS OF RANDOMIZED CONTROLLED TRIALS IN LIVER SURGERY.}

\section{Ascites ( $n=6)$}

1. abdominal output $>500 \mathrm{~mL} /$ day of ascites that required medical treatment to be controlled $^{2-5}$

2. abdominal drain output $>500 \mathrm{~mL} /$ day for more than 3 days ${ }^{6}$

3. continuous discharge of ascites (greater than $500 \mathrm{~mL} /$ day) from the drainage tube for longer than 10 days postoperatively ${ }^{7}$

\section{Intra-abdominal abscess $(n=6)$}

1. according to the Centres for Disease Control criteria for surgical site infections $\rightarrow$ presence of the following findings within 30 days after surgery: inflammatory findings, such as fever and flare, drainage of pus from the incision or drain, detection of a pathogen by culture of fluid or tissue sample and fluid retention on imaging indicating the presence of pus in a deep region ${ }^{8}$

2. purulent discharge with positive cultures from abdominal drains placed at surgery or fluid collection requiring a drainage procedure ${ }^{9,10}$

3. positive culture in the presence of clinical evidence of infection ${ }^{5,11}$

4. biliary communication together with purulent drainage ${ }^{12}$

\section{Bile leakage/biliary fistula $(n=10)$}

1. bilirubin concentration in the drain discharge $>10 \mathrm{mg} / \mathrm{dL}[171 \mu \mathrm{mol} / \mathrm{L}]$ for at least 3 days starting from the fifth postoperative day ${ }^{13}$

2. drainage of bile from the abdominal wound or drain; or intra-abdominal collection of bile confirmed at the time of re-operation or percutaneous drainage or cholangiography ${ }^{14}$

3. drain output $>100 \mathrm{~mL} /$ day for $>10$ days $^{15}$

4. postoperative biliary drainage through the abdominal drains ${ }^{12}$

5. $>1.5 \mathrm{mmol} / \mathrm{L}$ bile salts in drained fluids ${ }^{16}$

6. drainage of bile from the abdominal wound or drains with bilirubin content higher than the plasma levels; or intra-abdominal collection of bile at the time of re-operation or percutaneous drainage; or cholangiographic evidence of biliary leakage ${ }^{2}$

7. drainage of bile from the abdominal wound and drain, showing a total bilirubin level of $>5 \mathrm{mg} / \mathrm{dL}$ [85 $\mathrm{\mu mol} / \mathrm{L}$ ] or three times the serum level in the discharge fluid; or an intra-abdominal accumulation of bile confirmed by percutaneous drainage; or cholangiographic evidence of bile leakage ${ }^{8}$

8. bilirubin concentration in the drainage fluid $>5 \mathrm{mg} / \mathrm{dL}[85 \mu \mathrm{mol} / \mathrm{L}]^{17}$

9. total bilirubin level in drainage fluid on day 14 after surgery $>5 \mathrm{mg} / \mathrm{dL}[85 \mu \mathrm{mol} / \mathrm{L}]^{18}$ 
10. any drainage through the catheter with a bilirubin content higher than the plasma level $^{4}$

\section{Post-resectional liver failure/severe hepatic dysfunction $(n=11)$}

1. serum bilirubin level $>5 \mathrm{mg} / \mathrm{dL}[85 \mu \mathrm{mol} / \mathrm{L}]$ and/or prothrombin activity $<40 \mathrm{per}$ cent of normal for at least 3 postoperative days. Fatal liver failure was defined as death from irreversible hepatic dysfunction (hepatic coma, massive deterioration of blood coagulation, progressive hyperbilirubinemia) in the absence of other causes, such as sepsis ${ }^{14}$

2. bilirubin $>100 \mathrm{mg} /$ day for $>3$ days $\mathrm{s}^{15}$

3. prothrombin time $<50$ per cent of normal and/or serum bilirubin $>2.9 \mathrm{mg} / \mathrm{dL}$ [50 $\mu \mathrm{mol} / \mathrm{L}]$ on postoperative day 5 or thereafter and/or hepatic encephalopathy ${ }^{2,4}$

4. simultaneous presence of a prothrombin time $<50$ per cent of normal and a serum bilirubin $>2.9 \mathrm{mg} / \mathrm{dL}[50 \mu \mathrm{mol} / \mathrm{L}]$ on day 5 after surgery ${ }^{19}$

5. encephalopathy, ascites (volume $>500 \mathrm{~mL} /$ day), prothrombin time-international normalized ratio $>1.5$ on day 5 or $>2$ at any moment; or bilirubin level $>3 \mathrm{mg} / \mathrm{dL}$ [51 $\mu \mathrm{mol} / \mathrm{L}]$ on day $5^{5}$

6. serum bilirubin concentration $>5.0 \mathrm{mg} / \mathrm{dL}[85 \mu \mathrm{mol} / \mathrm{L}]$ or a prothrombin time $<50$ per cent of normal at 3 days or more after operation ${ }^{20}$

7. prothrombin time $<50$ per cent of normal and/or serum total bilirubin $>2.9 \mathrm{mg} / \mathrm{dL}$ [50 $\mathrm{\mu mol} / \mathrm{L}]$ and/or hepatic encephalopathy ${ }^{3}$

8. serum total bilirubin concentration $>5.3 \mathrm{mg} / \mathrm{dL}[90 \mu \mathrm{mol} / \mathrm{L}]$ or prothrombin time $<30$ per cent of normal level within 7 days of operation. Asterixis and alteration of consciousness not related to the effect of drugs were considered signs of liver failure, even when isolated ${ }^{6}$

9. elevation of serum total bilirubin $>2 \mathrm{mg} / \mathrm{dL}$ [34 $\mu \mathrm{mol} / \mathrm{L}]$ for longer than 10 days postoperatively ${ }^{7}$

10. total serum bilirubin 2-times higher than its normal level and massive ascites ${ }^{21}$

\section{Intra-abdominal hemorrhage $(n=1)$}

1. hemorrhage requiring re-operation ${ }^{6}$

\section{Mortality $(n=34)$}

1. in-hospital death $2,4,6,19-27$

a. death during the same period of hospitalization ${ }^{28}$

2. operative mortality $5,8,14,15,17,18,21,29-32$

a. any death resulting from a complication during surgery ${ }^{33}$

3. 30-day mortality $3,9-11,13,16,19,24,34$

4. 60-day mortality 22,35 
5. 90-day mortality ${ }^{13,36,37}$

\section{Wound infection $(n=4)$}

1. according to the criteria of Centres for Disease Control for surgical cite infections $\rightarrow$ the presence of the following findings within 30 days after surgery: inflammatory findings, such as fever and flare, drainage of pus from the incision or drain, detection of a pathogen by culture of fluid or tissue sample and fluid retention on imaging indicating the presence of pus in a deep region ${ }^{8}$

2. spontaneous or surgically released purulent discharge with positive cultures ${ }^{9,10}$

3. positive culture of fluids in the presence of clinical evidence of infection ${ }^{5}$

\section{Pneumonia $(n=4)$}

1. characteristic pulmonary infiltrate on a chest radiograph accompanied by leukocytosis ${ }^{9,10}$

2. positive culture of sputum in the presence of clinical evidence of infection ${ }^{5}$

3. a productive cough with new lung findings of coarse crepitations, large areas of bronchial breathing, or dullness to percussion (in the absence of an effusion) with supporting chest radiographs ${ }^{19}$

\section{Sepsis $(n=1)$}

1. temperature $\geq 38.5^{\circ} \mathrm{C}$, a white blood cell count $\geq 10,000$ cells $/ \mu \mathrm{L}$, and positive blood cultures or a documented septic focus ${ }^{6}$

\section{Acute renal failure $(n=1)$}

1. serum creatinine $>1.7 \mathrm{mg} / \mathrm{dL}[150 \mu \mathrm{mol} / \mathrm{L}]^{6}$ 


\section{APPENDIX 7. SURVEY ON DEFINITIONS OF COMPLICATIONS OF LIVER} SURGERY.

This survey consists of definitions of the most common complications of liver surgery in order to design a liver surgery specific composite endpoint (CEP). The survey is divided into two parts:

\section{Part I}

1. Definitions: the definitions were composed using definitions derived from literature. Please approve or disapprove these definitions. Whenever you disagree with a definition, please explain in the suggestion box. We would also like to ask your opinion on some controversies related to these definitions.

2. Liver surgery specific complications: you will be asked whether the complication is liver surgery specific or general.

\section{Part II}

1. Components of the liver surgery specific CEP: you will be asked to tell us which complications you would include in a liver surgery specific CEP.

\section{Part I}

\section{Pleural effusion}

Any fluid in pleural cavity proven by radiological imaging.

O I approve this definition

I do not approve this definition

I disapprove this definition because: .

\section{Discussion}

1. Do you consider pleural effusion a complication when the patient is not respiratory compromised?

O Yes

No

2. Do you consider pleural effusion a "normal" response to liver surgery?

Y Yes

No

Suggestion box: 
Pleural effusion is a:

$\bigcirc$ Liver surgery specific complication

$\bigcirc$ General complication

\section{Ascites}

Drainage of clear fluid in an amount greater than $500 \mathrm{~mL} /$ day via wound or intraabdominal drain or radiologically proven intra-abdominal clear fluid from postoperative day 3 onwards.

O I approve this definition

I do not approve this definition

I disapprove this definition because:

\section{Discussion}

1. Do you think "clear fluid" should be divided into transudate or exudate?

O Yes

O No

2. Do you check the nature of the fluid biochemically in your daily practice?

$\bigcirc$ Yes

O No

Suggestion box:

Ascites is a:

Liver surgery specific complication

General complication

\section{Wound infection ${ }^{38}$}

Criteria for defining a surgical site infection (SSI):

- Superficial incisional SSI: Infection occurs within 30 days after the operation and infection involves only skin or subcutaneous tissue of the incision and at least one of the following:

1. Purulent drainage, with or without laboratory confirmation, from the superficial incision.

2. Organisms isolated from an aseptically obtained culture of fluid or tissue from the superficial incision.

3. At least one of the following signs or symptoms of infection: pain or tenderness, localized swelling, redness, or heat, and superficial incision is deliberately opened by surgeon, unless incision is culture-negative. 
4. Diagnosis of superficial incisional SSI by the surgeon or attending physician.

- Deep incisional SSI: Infection occurs within 30 days after the operation and the infection appears to be related to the operation and infection involves deep soft tissues of the incision and at least one of the following:

1. Purulent drainage from the deep incision but not from the organ/space component of the surgical site.

2. A deep incision spontaneously dehisces or is deliberately opened by a surgeon when the patient has at least one of the following signs or symptoms: fever (temperature of more than $38^{\circ} \mathrm{C}$ ), localized pain, or tenderness, unless site is culture-negative.

3. An abscess or other evidence of infection involving the deep incision is found on direct examination, during re-operation, or by histopathological or radiological examination.

4. Diagnosis of a deep incisional SSI by a surgeon or attending physician.

I approve this definition

I do not approve this definition

I disapprove this definition because:

Wound infection is a:

$\bigcirc$ Liver surgery specific complication

General complication

Intra-abdominal abscess

Drainage of any quantity of purulent fluid from the abdominal drain; collection of pus in the abdominal cavity at the time of re-operation or percutaneous drainage. Fluid in drain or intra-abdominal collection should be culture-positive.

O I approve this definition

O I do not approve this definition

I disapprove this definition because:

\section{Discussion}

1. Do you agree the fluid should be culture-positive?

O Yes

O No

Suggestion box: 
Intra-abdominal abscess is a:

Liver surgery specific complication

General complication

\section{Bile leakage}

Drainage of any quantity of bile from the abdominal wound or drains at least $24 \mathrm{hr}$ postoperatively; intra-abdominal collection of bile at the time of re-operation or percutaneous drainage; cholangiographic evidence of contrast leakage. Fluid in drain or intra-abdominal collection should have a bilirubin content at least twice as high as bilirubin plasma concentration.

O I approve this definition

O I do not approve this definition

I disapprove this definition because:

\section{Discussion}

1. Is the addition "at least $24 \mathrm{hr}$ postoperatively" of extra value in this definition?

O Yes

O No

2. Do you think biochemical evidence of bilirubin in the drain or intra-abdominal collection is a prerequisite to meet the definition of bile leakage?

O Yes

O No

3. Do you check the presence of bilirubin in drain or intra-abdominal fluid in your daily practice when you suspect a bile leakage? If so, please write down in the suggestion box which bilirubin level is considered to reflect bile leakage.

O Yes

O No

Suggestion box:

Bile leakage is a:

$\bigcirc$ Liver surgery specific complication

O General complication

Sepsis 39

The clinical syndrome defined by the presence of both infection and a systemic inflammatory response. 
- Infection: pathological process caused by invasion of normally sterile tissue or fluid or body cavity by pathogenic or potentially pathogenic micro-organisms.

- Systemic inflammatory response syndrome (at least 2 criteria must be met):

1. Body temperature higher than $38^{\circ} \mathrm{C}$ or lower than $36^{\circ} \mathrm{C}$

2. Heart rate higher than $90 / \mathrm{min}$

3. Hyperventilation evidenced by respiratory rate higher than $20 / \mathrm{min}$ or arterial carbon dioxide partial pressure lower than $32 \mathrm{mmHg}$

4. White blood cell count higher than 12,000 cells/ $\mu \mathrm{L}$ or lower than 4,000 cells/ $\mu \mathrm{L}$

O I approve this definition

I do not approve this definition

I disapprove this definition because:

Sepsis is a:

Liver surgery specific complication

General complication

Post-resectional liver failure

Failure of one or more of the hepatic excretory and synthetic functions that include hyperbilirubinemia $>50 \mu \mathrm{mol} / \mathrm{L}$ (equivalent to $2.9 \mathrm{mg} / \mathrm{dL}$ ), prothrombin index $(\mathrm{PT})<50$ per cent (equal to an international standardized ratio > 1.7) and/or different grades of hepatic encephalopathy (HE) from postoperative day 3 onwards or at death.

Moderate post-resectional liver failure is defined when:
a. PT index is decreased and
b. hyperbilirubinemia is present and
c. HE is absent

Severe post-resectional liver failure is defined when:

a. PT index is decreased and

b. hyperbilirubinemia is present and

c. HE is present

O I approve this definition

O I do not approve this definition

I disapprove this definition because: .

\section{Discussion}

1. Do you think "from postoperative day 3 onwards" is of extra value in this definition?

$\mathrm{O}$ Yes

No 
2. Do you think other parameters reflecting liver synthetic or excretory function should be included in this definition? If yes, please insert these parameters in the suggestion box.

O Yes

O No

Suggestion box:

Post-resectional liver failure is a:

$\bigcirc$ Liver surgery specific complication

General complication

\section{Pneumonia $^{38}$}

Clinical entity meeting one of the following criteria:

1. Rales or dullness to percussion on physical examination of the chest and any of the following:

a. New onset of purulent sputum or change in character of sputum.

b. Organisms isolated from blood culture.

c. Isolation of pathogens from specimen obtained by transtracheal aspirate, broncheal brushing, or biopsy.

2. Chest radiographic examination shows new or progressive infiltrate, consolidation, cavitation, or pleural effusion and any of the following:

a. New onset of purulent sputum or change in character of sputum.

b. Organisms isolated from blood culture.

c. Isolation of pathogens from specimen obtained by transtracheal aspirate, broncheal brushing, or biopsy.

d. Isolation of virus or detection of viral antigen in respiratory secretions.

e. Diagnostic single antibody titer (IgM) or fourfold increase in paired serum samples (lgG) for pathogen.

f. Histopathological evidence of pneumonia.

O I approve this definition

O I do not approve this definition

I disapprove this definition because:

Pneumonia is a:

$\bigcirc$ Liver surgery specific complication

General complication 


\section{Intra-abdominal bleeding}

Leakage of blood via the abdominal drain with hemodynamic instability or asymptomatic bleeding that causes a collection of blood in proximity of the liver (hematoma) and that can be diagnosed radiologically.

O I approve this definition

O I do not approve this definition

I disapprove this definition because:

\section{Discussion}

1. Do you think we should specify the quantity of blood? If so please notify in the suggestion box how much blood you think will qualify for bleeding.

$\mathrm{O}$ Yes

O No

2. Do you think the hematoma should be radiologically proven?

O Yes

O No

Suggestion box:

Intra-abdominal bleeding is a:

Liver surgery specific complication

General complication

Acute renal failure ${ }^{40}$

An increase in serum creatinine of $0.5 \mathrm{mg} / \mathrm{dL}(44.2 \mu \mathrm{mol} / \mathrm{L})$ if baseline level is less than $2.5 \mathrm{mg} / \mathrm{dL}(221 \mu \mathrm{mol} / \mathrm{L})$ or an increase in serum creatinine by more than $20 \%$ if baseline level is more than $2.5 \mathrm{mg} / \mathrm{dL}(221 \mu \mathrm{mol} / \mathrm{L})$.

O I approve this definition

O I do not approve this definition

I disapprove this definition because:

Acute renal failure is a:

O Liver surgery specific complication

O General complication 


\section{Blood transfusion}

Requirement of any quantity of packed cells during surgery or after surgery.

O I approve this definition

I do not approve this definition

I disapprove this definition because:

\section{Discussion}

1. Do you think we should quantify the blood loss? If yes, please specify in the suggestion box what quantity would be required to complete the definition.

$O$ Yes

No

2. Do you agree we should extend the period of blood loss beyond the surgery time? If yes, please specify in the suggestion box how long blood loss should be recorded.

$\mathrm{O}$ Yes

O No

Suggestion box:

Requirement of blood transfusion is a:

Liver surgery specific complication

General complication

\section{Operative mortality}

Death of a patient directly related to liver surgery.

O I approve this definition

I do not approve this definition

I disapprove this definition because:

\section{Part II}

Now, we would like to ask you to inform us on the complications you think should be included as components of the liver surgery specific CEP. For your information, we provide some general characteristics of CEPs ${ }^{41}$.

1. A CEP is an outcome that captures the number of patients experiencing one or more complications.

2. The components of a CEP should be ascertainable without bias. 
3. The components of the CEP must be associated with the primary objective of a clinical trial (e.g. the effect of an intervention on surgery-related mortality and morbidity).

4. The validity of a CEP depends on similarity in

- clinical importance of the components from the patient's perspective (preferably: equal or nearly equal severity grade).

- effect of the intervention on the incidence of the component (preferably: equal or nearly equal effect size).

- incidence rate of each component (preferably: equal or nearly equal incidence rate).

Which complications depicted in the Table should be included in the liver surgery specific CEP according to you?

\begin{tabular}{l}
\hline Pes \\
Pleural effusion \\
Ascites \\
Wound infection \\
Intra-abdominal abscess \\
Bile leakage \\
Sepsis \\
Post-resectional liver failure \\
Pneumonia \\
Intra-abdominal bleeding \\
Acute renal failure \\
Requirement of blood transfusion \\
Operative mortality
\end{tabular}

Would you be interested in using a liver surgery specific CEP as primary endpoint of a randomized controlled trial?

O Yes

O No

Would you be willing to share your data from a previously conducted randomized controlled trial in liver surgery for determination of the current incidence rate and validation of the liver surgery specific CEP?

O Yes, contact me for further details

No, I'm not interested in sharing my data 


\section{APPENDIX 8. OVERVIEW OF RESPONDENTS TO THE WEB-BASED SURVEY.}

- Prof. dr. R Adam, Centre Hépato-Biliaire, Hôpital Paul Brousse, Villejuif, France

- Drs. M de Boer, Department of Surgery, University Medical Centre Groningen, Groningen, the Netherlands

- Prof. dr. I Borel Rinkes, Department of Surgery, University Medical Centre Utrecht, Utrecht, the Netherlands

- Prof. dr. O Busch, Department of Surgery, Academic Medical Centre, Amsterdam, the Netherlands

- Prof. dr. L Capussotti, Department of Surgery, Ospedale Mauriziano Umberto I, Torino, Italy

- Prof. dr. D Castaing, Centre Hépato-Biliaire, Hôpital Paul Brousse, Villejuif, France

- Prof. dr. PA Clavien, Department of Visceral and Transplantation Surgery, Swiss HPB Centre, Zürich, Switzerland

- Prof. dr. B Davidson, Department of Surgery, Royal Free Hospital, University College London, London, UK

- Prof. dr. C Dejong, Department of Surgery, Maastricht University Medical Centre, Maastricht, the Netherlands

- Prof. dr. J Figueras, Department of Surgery, Josep Trueta Hospital, Girona, Spain

- Dr. Y Fong, Division of Hepatopancreatobiliary Surgery, Memorial Sloan-Kettering Cancer Centre, New York, USA

- Prof. dr. O Garden, Department of Surgery, Royal Infirmary of Edinburgh, Edinburgh, UK

- Prof. dr. D Gouma, Department of Surgery, Academic Medical Centre, Amsterdam, the Netherlands

- Prof. dr. N Habib, Department of Surgery and Cancer, Imperial College, London, UK

- Prof. dr. R Hillegersberg, Department of Surgery, University Medical Centre Utrecht, Utrecht, the Netherlands

- Prof. dr. W Jarnagin, Division of Hepatopancreatobiliary Surgery, Memorial SloanKettering Cancer Centre, New York, USA

- Dr. J Klaase, Department of Surgery, Medical Spectrum Twente, Enschede, the Netherlands

- Prof. dr. P Lai, Department of Surgery, Prince of Wales Hospital, Chinese University of Hong Kong, Shatin, Hong Kong

- Prof. dr. H Lang, Department of General and Abdominal Surgery, Johannes Gutenberg-University Hospital, Mainz, Germany

- Prof. dr. W Lau, Faculty of Medicine, Chinese University of Hong Kong, Hong Kong, China 
- Dr. E Manusama, Department of Surgery, Medical Centre Leeuwarden, Leeuwarden, the Netherlands

- Prof. dr. P Neuhaus, Department of General, Visceral, and Transplantation Surgery, Charité, Campus Virchow-Klinikum, Humboldt University, Berlin, Germany

- Prof. dr. R Parks, Department of Surgery, Royal Infirmary of Edinburgh, Edinburgh, UK

- Prof. dr. R Porte, Department of Surgery, University Medical Centre Groningen, Groningen, the Netherlands.

- Prof. dr. A Revhaug, Surgical Research Laboratory, Institute of Clinical Medicine, University of Tromsö, Tromsö, Norway

- Dr. A Rijken, Department of Surgery, Amphia Hospital, Breda, the Netherlands

- Dr. R Roumen, Department of Surgery, Maxima Medical Centre Veldhoven, Veldhoven, the Netherlands

- Dr. J van der Sijp, Department of Surgery, Medical Centre Haaglanden, Den Haag, the Netherlands

- Dr. C Verhoef, Department of Hepatobiliary and Transplantation Surgery, Erasmus Medical Centre, Rotterdam, the Netherlands

- Prof. dr. SWigmore, Department of Surgery, Royal Infirmary of Edinburgh, Edinburgh, UK

- Prof. dr. J de Wilt, Department of Surgery, Radboud University Nijmegen Medical Centre, Nijmegen, the Netherlands 
APPENDIX 9. PRIMERS USED FOR QUANTITATIVE POLYMERASE CHAIN REACTION.

\begin{tabular}{lll}
\hline Name & Forward & Reverse \\
\hline PPIA $^{*}$ & CTCGAATAAGTTTGACTTGTGTTT & CTAGGCATGGGAGGGAACA \\
IL-1 $\beta$ & CTGAGCTCGCCAGTGAAATG & TTTAGGGCCATCAGCTTCAAA \\
IL-6 & TCCAGGAGCCCAGCTATGAA & GAGCAGCCCCAGGGAGAA \\
IL-8 & CTGGCCGTGGCTCTCTTG & TTAGCACTCCTTGGCAAAACTG \\
ICAM-1 & CTGAGCAATGTGCAAGAAGATAGC & CCCGTTCTGGAGTCCAGTACA \\
VCAM-1 & GCCGATCACAGTCAAGTGTTCA & CATGAGATGATCTCCTTTCAGTAAGTCT \\
\hline
\end{tabular}

* House-keeping gene Cyclo-A; IL, interleukin; ICAM-1, intercellular adhesion molecule; VCAM-1, vascular cell adhesion molecule. 


\section{APPENDIX 10. HEPATIC DAMAGE IN PATIENTS UNDERGOING LIVER SURGERY WITHOUT AND WITH INTERMITTENT PRINGLE MANEUVER.}

\begin{tabular}{|c|c|c|c|}
\hline & \multirow{2}{*}{$\begin{array}{l}\text { Control group } \\
\operatorname{nolPM}(n=10)\end{array}$} & \multicolumn{2}{|c|}{ Intervention groups } \\
\hline & & $15 \mathrm{IPM}(n=10)$ & $30 \mathrm{IPM}(n=10)$ \\
\hline \multicolumn{4}{|l|}{ L-FABP (ng/mL) } \\
\hline Baseline & $24 \pm 6$ & $24 \pm 8$ & $28 \pm 4$ \\
\hline Before mobilization & $409 \pm 206$ & $179 \pm 65$ & $316 \pm 162$ \\
\hline Before transection & $1102 \pm 111^{*}$ & $1229 \pm 279^{*}$ & $1573 \pm 516^{*}$ \\
\hline $30^{\prime}$ cumulative ischemia & $920 \pm 157^{*}$ & $762 \pm 170$ & $543 \pm 69^{*}$ \\
\hline $5^{\prime}$ reperfusion & $880 \pm 120^{*}$ & $1209 \pm 262$ & $2303 \pm 602^{*}$ \\
\hline After transection & $877 \pm 109^{*}$ & $1853 \pm 708^{*}$ & $3662 \pm 1355$ \\
\hline $8 \mathrm{hr}$ after start & $366 \pm 168$ & $791 \pm 221^{*}$ & $485 \pm 66^{*}$ \\
\hline POD1 & $146 \pm 28$ & $370 \pm 109$ & $496 \pm 108$ \\
\hline POD2 & $59 \pm 15$ & $137 \pm 44$ & $234 \pm 89$ \\
\hline POD3 & $25 \pm 7$ & $38 \pm 15$ & $42 \pm 9$ \\
\hline \multicolumn{4}{|l|}{ ALAT (IU/L) } \\
\hline Baseline & $34 \pm 9$ & $47 \pm 10$ & $27 \pm 5$ \\
\hline Before mobilization & $38 \pm 9$ & $48 \pm 9$ & $34 \pm 7$ \\
\hline Before transection & $119 \pm 18^{*}$ & $151 \pm 17^{*}$ & $132 \pm 24^{*}$ \\
\hline $30^{\prime}$ cumulative ischemia & $198 \pm 42^{*}$ & $159 \pm 27$ & $156 \pm 27^{*}$ \\
\hline $5^{\prime}$ reperfusion & $168 \pm 44$ & $179 \pm 29$ & $202 \pm 40^{*}$ \\
\hline After transection & $201 \pm 50$ & $280 \pm 80$ & $452 \pm 127$ \\
\hline $8 \mathrm{hr}$ after start & $289 \pm 78$ & $577 \pm 242^{*}$ & $701 \pm 245$ \\
\hline POD1 & $328 \pm 67^{*}$ & $708 \pm 244$ & $697 \pm 172^{*}$ \\
\hline POD2 & $265 \pm 65$ & $384 \pm 94$ & $859 \pm 433$ \\
\hline POD3 & $208 \pm 35$ & $449 \pm 128$ & $609 \pm 232$ \\
\hline \multicolumn{4}{|l|}{ ASAT (IU/L) } \\
\hline Baseline & $55 \pm 22$ & $38 \pm 8$ & $24 \pm 5$ \\
\hline Before mobilization & $44 \pm 13$ & $42 \pm 7$ & $30 \pm 6$ \\
\hline Before transection & $138 \pm 20$ & $169 \pm 22^{*}$ & $155 \pm 27^{*}$ \\
\hline 30 ' ischemia & $199 \pm 37$ & $189 \pm 31^{*}$ & $196 \pm 33^{*}$ \\
\hline $5^{\prime}$ reperfusion & $198 \pm 44$ & $210 \pm 33^{*}$ & $246 \pm 54^{*}$ \\
\hline After transection & $225 \pm 50$ & $296 \pm 72^{*}$ & $510 \pm 129$ \\
\hline $8 \mathrm{hr}$ after start & $255 \pm 35^{*}$ & $590 \pm 232^{*}$ & $861 \pm 275$ \\
\hline POD1 & $391 \pm 89^{*}$ & $756 \pm 260^{*}$ & $837 \pm 219^{*}$ \\
\hline POD2 & $202 \pm 26$ & $276 \pm 46^{*}$ & $852 \pm 262$ \\
\hline POD3 & $112 \pm 16$ & $218 \pm 52$ & $236 \pm 59$ \\
\hline
\end{tabular}

Values are mean \pm standard error; 15IPM, 15 minutes intermittent Pringle maneuver; 30 IPM, 30 minutes intermittent Pringle maneuver; noIPM, no intermittent Pringle maneuver; L-FABP, liver fatty acid-binding protein; ALAT, alanine aminotransferase; ASAT, aspartate aminotransferase; POD, postoperative day; ${ }^{*}$ repeated measures two-way ANOVA with post-hoc paired sample t-test versus baseline: $p<0.050$. 


\section{REFERENCES}

1. Kirkwood BR. Essentials of medical statistics. Oxford: Blackwell Scientific Publications; 1988.

2. Figueras J, Llado L, Miro M, Ramos E, Torras J, Fabregat J, et al. Application of fibrin glue sealant after hepatectomy does not seem justified: results of a randomized study in 300 patients. Ann Surg 2007; 245: 536-42.

3. Chen XP, Zhang ZW, Zhang BX, Chen YF, Huang ZY, Zhang WG, et al. Modified technique of hepatic vascular exclusion: effect on blood loss during complex mesohepatectomy in hepatocellular carcinoma patients with cirrhosis. Langenbecks Arch Surg 2006; 391: 209-15.

4. Figueras J, Llado L, Ruiz D, Ramos E, Busquets J, Rafecas A, et al. Complete versus selective portal triad clamping for minor liver resections: a prospective randomized trial. Ann Surg 2005; 241: 582-90.

5. Aldrighetti L, Pulitano C, Arru M, Finazzi R, Catena M, Soldini L, et al. Impact of preoperative steroids administration on ischemia-reperfusion injury and systemic responses in liver surgery: a prospective randomized study. Liver Transp/ 2006; 12: 941-9.

6. Azoulay D, Lucidi V, Andreani P, Maggi U, Sebagh M, Ichai P, et al. Ischemic preconditioning for major liver resection under vascular exclusion of the liver preserving the caval flow: a randomized prospective study. J Am Coll Surg 2006; 202: 203-11.

7. Yoshida H, Mamada Y, Taniai N, Mizuguchi Y, Shimizu T, Takahashi T, et al. Fixation of the greater omentum for prevention of delayed gastric emptying after left-sided hepatectomy: a randomized controlled trial. Hepatogastroenterology 2005; 52: 1334-7.

8. Togo S, Tanaka K, Matsuo K, Nagano Y, Ueda M, Morioka D, et al. Duration of antimicrobial prophylaxis in patients undergoing hepatectomy: a prospective randomized controlled trial using flomoxef. J Antimicrob Chemother 2007; 59: 964-70.

9. Sugawara G, Nagino M, Nishio H, Ebata T, Takagi K, Asahara T, et al. Perioperative synbiotic treatment to prevent postoperative infectious complications in biliary cancer surgery: a randomized controlled trial. Ann Surg 2006; 244: 706-14.

10. Kanazawa H, Nagino M, Kamiya S, Komatsu S, Mayumi T, Takagi K, et al. Synbiotics reduce postoperative infectious complications: a randomized controlled trial in biliary cancer patients undergoing hepatectomy. Langenbecks Arch Surg 2005; 390: 104-13.

11. Pulitano C, Aldrighetti L, Arru M, Finazzi R, Soldini L, Catena M, et al. Prospective randomized study of the benefits of preoperative corticosteroid administration on hepatic ischemia-reperfusion injury and cytokine response in patients undergoing hepatic resection. HPB (Oxford) 2007; 9: 183-9.

12. Yuksel O, Akyurek N, Sahin T, Salman B, Azili C, Bostanci H. Efficacy of radical surgery in preventing early local recurrence and cavity-related complications in hydatic liver disease. J Gastrointest Surg 2008; 12: 483-9.

13. Torzilli G, Donadon M, Marconi M, Procopio F, Palmisano A, Del Fabbro D, et al. Monopolar floating ball versus bipolar forceps for hepatic resection: a prospective randomized clinical trial. $J$ Gastrointest Surg 2008; 12: 1961-6.

14. Heizmann O, Loehe F, Volk A, Schauer RJ. Ischemic preconditioning improves postoperative outcome after liver resections: a randomized controlled study. Eur J Med Res 2008; 13: 79-86.

15. Nordlinger B, Sorbye H, Glimelius B, Poston GJ, Schlag PM, Rougier P, et al. Perioperative chemotherapy with FOLFOX4 and surgery versus surgery alone for resectable liver metastases from colorectal cancer (EORTC Intergroup trial 40983): a randomised controlled trial. Lancet 2008; 371: 1007-16. 
16. Campagnacci R, De Sanctis A, Baldarelli M, Di Emiddio M, Organetti L, Nisi M, et al. Hepatic resections by means of electrothermal bipolar vessel device (EBVS) LigaSure V: early experience. Surg Endosc 2007; 21: 2280-4.

17. Esaki M, Sano T, Shimada K, Sakamoto Y, Takahashi Y, Wakai K, et al. Randomized clinical trial of hepatectomy using intermittent pedicle occlusion with ischaemic intervals of 15 versus 30 minutes. Br J Surg 2006; 93: 944-51.

18. Arita J, Hasegawa K, Kokudo N, Sano K, Sugawara Y, Makuuchi M. Randomized clinical trial of the effect of a saline-linked radiofrequency coagulator on blood loss during hepatic resection. $\mathrm{Br} J$ Surg 2005; 92: 954-9.

19. Pessaux P, Regimbeau JM, Dondero F, Plasse M, Mantz J, Belghiti J. Randomized clinical trial evaluating the need for routine nasogastric decompression after elective hepatic resection. $\mathrm{Br} J$ Surg 2007; 94: 297-303.

20. Capussotti L, Muratore A, Ferrero A, Massucco P, Ribero D, Polastri R. Randomized clinical trial of liver resection with and without hepatic pedicle clamping. Br J Surg 2006; 93: 685-9.

21. Li SQ, Liang LJ, Huang JF, Li Z. Ischemic preconditioning protects liver from hepatectomy under hepatic inflow occlusion for hepatocellular carcinoma patients with cirrhosis. World J Gastroenterol 2004; 10: 2580-4.

22. Jarnagin WR, Gonen M, Maithel SK, Fong Y, D'Angelica MI, Dematteo RP, et al. A prospective randomized trial of acute normovolemic hemodilution compared to standard intraoperative management in patients undergoing major hepatic resection. Ann Surg 2008; 248: 360-9.

23. Kim YI, Fujita S, Hwang YJ, Chun JM, Song KE, Chun BY. Successful intermittent application of the Pringle maneuver for 30 minutes during human hepatectomy: a clinical randomized study with use of a protease inhibitor. Hepatogastroenterology 2007; 54: 2055-60.

24. Shi M, Guo RP, Lin XJ, Zhang YQ, Chen MS, Zhang CQ, et al. Partial hepatectomy with wide versus narrow resection margin for solitary hepatocellular carcinoma: a prospective randomized trial. Ann Surg 2007; 245: 36-43.

25. Liu CL, Fan ST, Cheung ST, Lo CM, Ng IO, Wong J. Anterior approach versus conventional approach right hepatic resection for large hepatocellular carcinoma: a prospective randomized controlled study. Ann Surg 2006; 244: 194-203.

26. Sun HC, Qin LX, Lu L, Wang L, Ye QH, Ren N, et al. Randomized clinical trial of the effects of abdominal drainage after elective hepatectomy using the crushing clamp method. Br J Surg 2006; 93: 422-6.

27. Wu CC, Ho WM, Cheng SB, Yeh DC, Wen MC, Liu TJ, et al. Perioperative parenteral tranexamic acid in liver tumor resection: a prospective randomized trial toward a "blood transfusion"-free hepatectomy. Ann Surg 2006; 243: 173-80.

28. Liu CL, Fan ST, Lo CM, Wong Y, Ng IO, Lam CM, et al. Abdominal drainage after hepatic resection is contraindicated in patients with chronic liver diseases. Ann Surg 2004; 239: 194-201.

29. Kim Yl, Chung HJ, Song KE, Hwang YJ, Lee JW, Lee YJ, et al. Evaluation of a protease inhibitor in the prevention of ischemia and reperfusion injury in hepatectomy under intermittent Pringle maneuver. Am J Surg 2006; 191: 72-6.

30. Smyrniotis V, Arkadopoulos N, Kostopanagiotou G, Farantos C, Vassiliou J, Contis J, et al. Sharp liver transection versus clamp crushing technique in liver resections: a prospective study. Surgery 2005; 137: 306-11.

31. Lodge JP, Jonas S, Oussoultzoglou E, Malago M, Jayr C, Cherqui D, et al. Recombinant coagulation factor VIla in major liver resection: a randomized, placebo-controlled, double-blind clinical trial. Anesthesiology 2005; 102: 269-75. 
32. Nuzzo G, Giuliante F, Vellone M, De Cosmo G, Ardito F, Murazio M, et al. Pedicle clamping with ischemic preconditioning in liver resection. Liver Transp/ 2004; 10: S53-7.

33. Saiura A, Yamamoto J, Koga R, Sakamoto Y, Kokudo N, Seki M, et al. Usefulness of LigaSure for liver resection: analysis by randomized clinical trial. Am J Surg 2006; 192: 41-5.

34. Kostopanagiotou G, Pandazi A, Matsota P, Arkadopoulos N, Dalamanga N, Politou M, et al. Effect of packed red blood cells transfusion on plasma fibronectin during liver resection. Transfus Med 2007; 17: 115-8.

35. Schwartz M, Madariaga J, Hirose R, Shaver TR, Sher L, Chari R, et al. Comparison of a new fibrin sealant with standard topical hemostatic agents. Arch Surg 2004; 139: 1148-54

36. Petrowsky H, McCormack L, Trujillo M, Selzner M, Jochum W, Clavien PA. A prospective, randomized, controlled trial comparing intermittent portal triad clamping versus ischemic preconditioning with continuous clamping for major liver resection. Ann Surg 2006; 244: 921-8.

37. Lesurtel M, Selzner M, Petrowsky H, McCormack L, Clavien PA. How should transection of the liver be performed?: a prospective randomized study in 100 consecutive patients: comparing four different transection strategies. Ann Surg 2005; 242: 814-22, discussion 822-3.

38. Garner JS, Jarvis WR, Emori TG, Horan TC, Hughes JM. CDC definitions for nosocomial infections, 1988. Am J Infect Control 1988; 16: 128-40.

39. Levy MM, Fink MP, Marshall JC, Abraham E, Angus D, Cook D, et al. 2001 SCCM/ESICM/ACCP/ATS/ SIS International Sepsis Definitions Conference. Crit Care Med 2003; 31: 1250-6.

40. Singri N, Ahya SN, Levin ML. Acute renal failure. Jama 2003; 289: 747-51.

41. Montori VM, Permanyer-Miralda G, Ferreira-Gonzalez I, Busse JW, Pacheco-Huergo V, Bryant D, et al. Validity of composite end points in clinical trials. Bmj 2005; 330: 594-6. 
Scientific output 



\section{PUBLISHED PAPERS}

1. van den Broek MA, Vreuls $C P$, Winstanley $A$, Jansen $R L$, van Bijnen $A A$, Dello $S A$, Bemelmans $\mathrm{MH}$, Dejong $\mathrm{CH}$, Driessen A, Olde Damink SW. Hyaluronic acid as a marker of hepatic sinusoidal obstruction syndrome secondary to oxaliplatin-based chemotherapy in patients with colorectal liver metastases. Ann Surg Oncol 2013: in press.

2. van den Broek MA, Shiri-Sverdlov R, Schreurs JW, Bloemen JG, Bieghs V, Rensen SS, Dejong $\mathrm{CH}$, Olde Damink SW. Liver manipulation during liver surgery in humans is associated with hepatocellular damage and hepatic inflammation. Liver Int 2013; in press.

3. Vreuls CP, Olde Damink SW, Koek GH, Winstanley A, Wisse E, Cloots R, van den Broek MA, Dejong CH, Bosman F, Driessen A. Glutathione S-transferase M1-null genotype as a risk factor for sinusoidal obstruction syndrome in oxaliplatin-treated patients with metastatic colorectal cancer. Br J Cancer 2013; in press.

4. Dello SA, Lodewick TM, van Dam RM, Reisinger KW, van den Broek MA, von Meyenfeldt MF, Bemelmans MH, Olde Damink SW, Dejong CH. Sarcopenia negatively affects preoperative total functional liver volume in patients undergoing liver resection. HPB 2013: 15: 165-9.

5. Vreuls $C P$, van den Broek MA, Winstanley $A$, Koek GH, Olde Damink SW, Wisse E, Dejong $\mathrm{CH}$, Bosman FT, Driessen A. Hepatic sinusoidal obstruction syndrome (SOS) reduces the effect of oxaliplatin in colorectal liver metastases. Histopathology 2012; 61: 314-8. Comment in: Histopathology 2012; 61: 1247-8.

6. Ezzat T, van den Broek MA, Davies N, Dejong CH, Bast A, Malagó M, Dhar DK, Olde Damink SW. The flavonoid monoHER prevents monocrotaline-induced hepatic sinusoidal injury in rats. J Surg Oncol 2012; 106: 72-8. Comment in: J Surg Oncol 2012; in press. Author reply in: J Surg Oncol 2012; in press.

7. Dello SA, Reisinger KW, van Dam RM, Bemelmans MH, van Kuppevelt $\mathrm{TH}$, van den Broek MA, Olde Damink SW, Poeze M, Buurman WA, Dejong $\mathrm{CH}$. Total intermittent Pringle maneuver during liver resection can induce intestinal epithelial cell damage and endotoxemia. Plos One 2012; 7: e30539.

8. van den Broek MA, van Dam RM, van Breukelen GJ, Bemelmans MH, Oussoultzoglou E, Pessaux P, Dejong CH, Freemantle N, Olde Damink SW. Development of a composite endpoint for randomized controlled trials in liver surgery. Br J Surg 2011; 98: 1138-45. Comment in: Br J Surg 2011; 98: 1145-6.

9. van den Broek MA, Bloemen JG, Dello SA, van de Poll MC, Olde Damink SW, Dejong $\mathrm{CH}$. Randomized controlled trial analyzing the effect of 15 or 30 min intermittent Pringle maneuver on hepatocellular damage during liver surgery. J Hep 2011; 55: 337-45. 
10. Dello SA, Bloemen JG, van de Poll MC, van Dam RM, Stoot JH, van den Broek MA, Buurman WA, Bemelmans $\mathrm{MH}$, Olde Damink SW, Dejong $\mathrm{CH}$. Gut and liver handling of interleukin-6 during liver resection in man. HPB 2011; 13: 324-31.

11. Mpabanzi L, van den Broek MA, Visschers RG, van de Poll MC, Nadalin S, Saner $\mathrm{FH}$, Dejong $\mathrm{CH}$, Malagó $\mathrm{M}$, Olde Damink SW. Urinary ammonia excretion increases acutely during living donor liver transplantation. Liver Int 2011; 31: 1150-4.

12. van der Vorst JR, van Dam RM, van Stiphout RS, van den Broek MA, Hollander IH, Kessels AG, Dejong $\mathrm{CH}$. Virtual liver resection and volumetric analysis of the future liver remnant using open source image processing software. World J Surg 2010; 34: 2426-33.

13. Wisse E, Braet F, Duimel H, Vreuls C, Koek G, Olde Damink SW, van den Broek MA, de Geest B, Dejong CH, Tateno C, Frederik P. Fixation methods for electron microscopic of human and other liver. World J Gastroenterol 2010; 16: 2851-66.

14. van den Broek MA, Olde Damink SW, Winkens B, Broelsch CE, Malagó M, Paul A, Saner $\mathrm{FH}$. Procalcitonin as prognostic marker for infectious complications in liver transplant recipients in an intensive care unit. Liver Transp/ 2010; 16: 402-10.

15. van den Broek MA, Olde Damink SW, Driessen A, Dejong $\mathrm{CH}$, Bemelmans MH. Nodular regenerative hyperplasia secondary to neoadjuvant chemotherapy for colorectal liver metastases. Case Reports Med 2009; 2009: 457975.

16. van den Broek MA, van Dam RM, Malagó $\mathrm{M}$, Dejong $\mathrm{CH}$, van Breukelen GJ, Olde Damink SW. Feasibility of randomized controlled trials in liver surgery using surgeryrelated mortality or morbidity as endpoint. Br J Surg 2009; 96: 1005-1014. Erratum in: Br J Surg 2010; 97: 302. Comment in: Br J Surg 2010; 97: 136. Author reply in: Br J Surg 2010; 97: 136-7.

17. van den Broek MA, Nijenhuis RJ, Backes WH, Teijink JA. Neurological deficit secondary to spinal cord ischemia after infrarenal abdominal aortic repair for aortoiliac occlusive disease: a case report. Eur J Vasc Endovasc Surg Extra 2009; 17: 29-31.

18. van den Broek MA, Olde Damink SW, Dejong CH, Lang H, Malagó M, Jalan R, Saner FH. Liver failure after partial hepatic resection: definition, pathophysiology, risk factors and treatment. Liver Int 2008; 28: 767-80.

19. Saner FH, Olde Damink SW, Pavlakovic G, van den Broek MA, Sotiropoulos GC, Radtke A, Paul A, Nadalin S, Malagó M, Broelsch CE. Positive end-expiratory pressure induces liver congestion in living donor liver transplant patients: myth or fact? Transplantation 2008; 85: 1863-6.

20. Saner FH, Olde Damink SW, Pavlakovic G, van den Broek MA, Rath PM, Sotiropoulos GC, Radtke A, Canbay A, Paul A, Nadalin S, Malagó M, Broelsch CE. Pulmonary and blood stream infections in adult living donor and cadaveric liver transplant patients. Transplantation 2008; 85: 1564-8. 
21. Nadalin S, Heuer M, Wallot M, Auth M, Schaffer R, Sotiropoulos GC, Ballauf A, van den Broek MA, Olde Damink SW, Hoyer PF, Broelsch CE, Malagó M. Paediatric acute liver failure and transplantation: the University of Essen experience. Transpl Int 2007; 20: 519-27.

22. Nijhuis J, van Dielen FM, Fouraschen SM, van den Broek MA, Rensen SS, Buurman WA, Greve JW. Endothelial activation markers and their key regulators after restrictive bariatric surgery. Obesity 2007; 15: 1395-9.

\section{SUBMITTED MANUSCRIPTS}

23. Pessaux , van den Broek MA, Marzano E, Olde Damink SW, Piardi T, Dejong $\mathrm{CH}_{\text {, }}$ Ntourakis D, van Dam RM. Identification and validation of risk factors for postoperative infectious complications following hepatectomy. Submitted with revisions.

24. Bloemen JG, van den Broek MA, Venema K, Buurman WA, Dejong CH. Randomized placebo controlled study of the effects of butyrate enemas on systemic concentrations and splanchnic fluxes of short chain fatty acids. Submitted.

25. Bloemen JG, van der Vorst JR, van den Broek MA, Venema K, Buurman WA, Dejong $\mathrm{CH}$. Effects of liver resection on hepatic short chain fatty acid handling in humans. Submitted.

\section{MANUSCRIPTS IN PREPARATION}

26. van Dam RM, Lodewick TM, van den Broek MA, de Jong MC, Greve JW, Jansen RL, Bemelmans $\mathrm{MH}$, Olde Damink SW, Dejong $\mathrm{CH}$. Outcomes of contemporary versus classical indications for patients undergoing liver surgery for colorectal cancer liver metastases.

27. van den Broek MA, Olde Damink SW, Adamzik M, Dejong CH, Broelsch CE, Paul A, Malagó M, Saner FH. Gene polymorphisms and the risk of infection and infectionrelated mortality in living donor liver transplant recipients. 

Dankwoord 

"Ons proefschrift is af!". Dit is geen uiting van koninklijk meervoud, maar een blijk van waardering voor alle mensen die door de jaren heen hebben bijgedragen aan de totstandkoming ervan. Een aantal van deze mensen wil ik graag persoonlijk noemen.

Allereerst mijn promotor, prof. dr. Dejong, beste Kees, als een ware pater familias waakte jij over het wel en wee van je onderzoeksgroep, waardoor er een uniek werkklimaat ontstond waarin iedereen kon stralen. Ik prijs me gelukkig één van jouw promovendi te zijn! Bedankt voor je wetenschappelijke adviezen, je deur die altijd openstond, je stichtelijke woorden in tijden van crisis, je optimisme, je nimmer aflatende steun, je klinische tips, je fantastische verhalen aan/op de bar en voor zoveel meer. Het begeleiden van promovendi is in jouw ogen een kwestie van zaaien en oogsten, net zoals het maken van goede wijn... de tijd om van de oogst te genieten is aangebroken: proost en merci pour tout!

Mijn copromotor, dr. Olde Damink, beste Steven, je overtuigde me, nadat ik als ANIOS aan de geneugten van de kliniek had geroken, om aan dit promotietraject te beginnen. Onze complementaire karakters hebben tot veel verhitte discussies geleid, maar waren ook de sleutel tot ons succes! Ik ben je dankbaar voor de vrijheid om mijn promotietraject, als ware het mijn eigen BV, vorm te geven, je actieve support bij het opzetten van internationale samenwerkingsverbanden, je tomeloze inzet voor onze wetenschappelijke parels, en de buitenkans om me op persoonlijk vlak te ontwikkelen in de Nationale DenkTank.

Ook de overige leden van het MUMC HPB team mogen in de eerste alinea's van dit dankwoord niet ontbreken:

Drs. van Dam, beste Ronaldo, de belangrijkste levenslessen heb ik tijdens deze reis van jou geleerd. Een greep uit de passies die we delen: 10-10-10, Het Champagne Comité, de 5 kamers, de 20-80 regel, de bank der wederdiensten, McMUMCey, de leverdatabase, de one-night paper, de van Dam tot Dam loop (gelukkig hebben we de foto's nog...), Bergen aan Zee, Room 1528, de 4 o'clock break en ga zo maar door. Het doel is gehaald. I owe you big time!

Dr. Bemelmans, beste Marc, jij zorgde voor de broodnodige ontspanning en relativering in onderzoeksland en daarom glipte ik vaak even bij je binnen! Ook in roerige tijden kon ik bij je terecht en was je een ware rots in de branding: ik ben je dankbaar voor je rol als bruggenbouwer en timemanager. Vooral je strakke deadlines, die met dodelijk serieuze blik werden aangekondigd toen de vaart uit het project dreigde te raken, hebben me ongelooflijk op weg geholpen. Ik hoop dat we nog vaak samen in een bar zullen belanden om onder het genot van een kleine alcoholische versnapering te filosoferen over het leven!

Dr. Stoot, beste Jan, in 2007 sloot jij je, als afgevaardigde uit de Leidsche regio, aan bij het MUMC HPB team. Wat een mooie aanwinst! Tijdens onze gezamenlijke tripjes 
naar congressen in verre oorden zorgde jij voor goede gesprekken en veel hilariteit. Met recht verdiende je de titel "star of the show"! Bedankt voor alle gezelligheid en ik hoop dat we nog veel mooie congressen zullen bezoeken in de toekomst.

Het merendeel van de studies beschreven in dit proefschrift is verricht in samenwerking met verschillende disciplines gelieerd aan diverse nationale en internationale centra. Door deze samenwerking werd het geheel daadwerkelijk meer dan de som der delen. Het is onmogelijk om iedereen hier persoonlijk te bedanken, een aantal mensen zal ik er uitlichten, maar voor iedereen die niet expliciet benoemd wordt: dank voor jullie input!

Prof. dr. Malagó, dear Max, I still feel lucky that we met in the operating theatre of Essen University Hospital. Thanks for taking me under your wings and supporting me throughout my PhD career that started off in Essen, Germany, and ended up in London, UK, via my home base Maastricht. I admire your determination, strong personality and ability to pay attention to all the members of your team despite your own overfull agenda. It was only after I met you that the word "magnanimous" took on a new meaning!

Dr. Saner, dear Fuat, you gave me the opportunity to study the living donor liver transplantation cohort of Essen University Hospital, which lead to a fruitful collaborative project and many publications! Prof dr. Broelsch, prof dr. Lang and dr. Nadalin, I greatly appreciate your support during my stay in Essen.

Dr. Dhar, dear Dipok, I would have been nowhere without your hands-on laboratory experience, your devotion to the monoHER story and your passion for research. Thanks for your supervision while working in the surgical laboratory of University College London Hospitals. Dr. Ezzat, dear Tarek, you were closely involved in planning and executing the monoHER experiments after I started my surgical training. I greatly appreciate your input and good luck with your own surgical career!

Dr. van Breukelen, beste Gerard, toen ik op een ochtend bij je binnenstapte om advies te vragen over een power berekening, konden wij allebei niet voorzien waar onze samenwerking toe zou leiden! Jouw rekenwerk en statische adviezen waren van cruciaal belang voor de artikelen uit deel I van dit proefschrift. Dank!

Dr. Jansen, beste Rob, als medisch oncoloog werd je meegezogen in ons enthousiasme voor "the blue liver". Dank voor de ruimte die je me gaf om als chirurgijn studies te verrichten op medisch oncologisch vlak. Ook dank aan de oncologen uit Venlo, Roermond, Weert, Sittard en Heerlen voor de toegang tot de patiëntendata beschreven in de hoofdstukken 8 en 9.

Dr. Driessen en drs. Vreuls, beste Ann en Celien, jullie bijdrage was onmisbaar voor het laatste deel van dit proefschrift. Dank voor de histologische beoordeling van de leverschade in de MUMC patiënten. Dr. Winstanley, dear Ali, thanks for being second observer and grading all the liver biopsies in no time. 
Dr. Shiri-Sverdlov, beste Ronit, toen ik al in het Westen zat, ben jij met je groep verder gegaan met het analyseren van de humane leverbiopsiën. Dank voor de input die heeft geleid tot de totstandkoming van hoofdstuk 5 .

Tevens wil ik de volgende mensen, die tijdens mijn promotieonderzoek een belangrijke bijdrage hebben geleverd aan mijn wetenschappelijke of persoonlijke vorming, bedanken: prof. dr. Buurman en prof. dr. Stassen (Afdeling Algemene Heelkunde, MUMC), dr. Winkens (Afdeling Statistiek en Methodologie, UM), prof. dr. Bast (Afdeling Farmacologie en Toxicologie, UM), prof. dr. Wisse en Hans Duimel (Afdeling Electronen Microscopie, UM), prof. dr. Freemantle (Department of Primary Care and General Practice, University of Birmingham, UK), prof. dr. Jalan (Institute of Hepatology, University College London and Hospitals and the Royal Free Hospital, UK), en prof. dr. Pessaux (Department of HPB Surgery and Liver Transplantation, Hautepierre Hospital, France).

Veel dank ben ik ook verschuldigd aan de beoordelingscommissie voor hun vakkundige oordeel: prof. dr. van Kleef, prof. dr. van Engeland, prof. dr. Farges, prof. dr. van der Hulst, en prof. dr. Tollenaar.

Een speciaal woord van dank aan alle patiënten die hebben ingestemd met deelname aan de studies die beschreven worden in dit proefschrift. Zonder dat zij daar zelf direct beter van werden, hebben ze bijgedragen aan de voortgang van de medische wetenschap. Ook dank aan alle operatie- en anesthesieassistenten van het MUMC voor de medewerking bij het verzamelen van de honderden samples op de operatiekamer.

En dan vanzelfsprekend mijn onderzoekscollega's. Johanne (beter bekend als mijn zeer begaafde tweelingzus genaamd JB, bloempje, jut, snap, knabbel, stClaire of Jozef): de humane leverchirurgie studies hebben we samen verricht onder het motto "zonder dalen, geen pieken". Wij weten wat reanimeren is... Maar naast de wetenschap waren daar ook bieren in de Thembi's, (après-) skiën in Sankt Anton, party(crash)en in Maastricht, flaneren door Londen en feesten in Athene. Zowel collega's als echte vrienden. Het blijft jammer dat we niet in driedelig wit door hetzelfde ziekenhuis struinen! Gelukkig bied je altijd een luisterend oor als ik in het Westen weer eens bezig ben een wetenschappelijke of klinische uitdaging te overwinnen. Wie anders dan jij zou mijn "ene" paranimf moeten zijn?

Mijn roomies van de 3/1: allereerst Ruben, studie- en kamergenoot van het eerste uur. Vier jaar lang hebben we, eerst zij aan zij en daarna back to back, gewerkt aan de totstandkoming van onze pareltjes. Ook al verschillen onze karakters totaal, ik kan toch met recht zeggen dat er maar weinigen waren die mij zo goed konden peilen als jij. Ik heb ontzettend veel respect voor jouw onverwoestbare doorzettingsvermogen en 
ongelooflijk stabiel neutrale $\mathrm{pH}$. Om onze samenwerking in stijl te bezegelen: daanke jong en veer zien us!

Kirsten alias Frau Huntjens: we are sure we want to close SPSS! Wat hebben we ellenlang zitten zwoegen op onze databases en bijna-voltooide-maar-het-moet-tochecht-nog-net-even-anders manuscripten. Gedeelde smart was halve smart... Maar ook je steun en motiverende woorden tijdens de afrondende fase van mijn proefschrift, toen we allebei al in de Randstad woonden en werkten, waren voor mij erg belangrijk. Wat fijn dat we de vrijdagmiddagborrel gewoon voort kunnen zetten in onze nieuwe Thembi's in de Residentie!

ArgiNina, Lianne en Mascha, onze vele gezamenlijke kopjes koffie in ons bijzondere kantoor op de 3 zorgden voor de onmisbare ontspanning na (maar vaak ook voor) wetenschappelijke inspanning. Simon, ik ben je dankbaar voor je hulp bij het verzamelen, beheren en analyseren van al het patiëntenmateriaal. Mechteld, jij nam mijn bureau en plaats in het MUMC HPB team met veel succes over. Jammer dat we niet aan samenwerken zijn toegekomen, want onze bezoekjes aan de IHPBA congressen in Buenos Aires en Kaapstad smaakten zeker naar meer!

Tot slot ben ik natuurlijk ook alle collega's van de 5, in het bijzonder de vaste steunpilaren Annemarie, Bas, Hans, Kaatje, Mo, Sander en (chef reservebank) Tim, en de studenten die hebben bijgedragen aan de datacollectie van een aantal manuscripten, ontzettend dankbaar.

Dank ook aan de Heerlense boys, onder aanvoering van Dr. Welten, die mij als coassistent en later als ANIOS hebben geholpen bij mijn eerste klinische stappen binnen de Heelkunde. Jullie motto "no guts, no glory" komt nog dagelijks van pas!

En natuurlijk mijn Leiderdorpse collega's, het was spannend om in een nieuwe regio aan mijn opleiding tot chirurg te beginnen. Mede door de geweldige sfeer in de assistenten- en opleidersgroep voelde ik me vanaf de eerste dag thuis in het Rijnland Ziekenhuis. Ongelooflijk bedankt voor de klinische tips and tricks, de duwtjes in de rug op momenten dat ik ze nodig had en, niet te vergeten, de mooie borrels, BBQ's en feestjes!

Wie en waar zou ik zijn zonder mijn vrienden? Het "project-not-to-be-named" is eindelijk af! Er is weer tijd voor leuke dingen: winen en dinen, hardlopen, shoppen, flaneren langs het strand, reizen en ga zo maar door.

Klaar en Monique, we zijn met z'n drieën aan onze studententijd in Maastricht begonnen. We hebben samen, zowel letterlijk als figuurlijk, vele hoogtepunten beleefd en ik hoop dat er ook nog vele zullen volgen. Klaar en Hans, ik geniet van de eindeloze diners en logeerpartijtjes in de wereldstad Vleuten. Fijn om te weten dat ik altijd bij jullie kan aankloppen! Monique, jouw humor en nuchtere blik op het leven brengen mij altijd weer met beide benen op de grond en zijn daarom erg belangrijk voor mij. 
Nanette, DB'er in hart en nieren, mijn dank voor alle goed-foute adviezen, shop-tillwe-drop momenten, relatieadviezen, verkleedpartijtjes, springfoto's, en TY beautytips is onbeschrijflijk groot! En wat fijn dat ik ook altijd voor "serious business" bij je terecht kan.

Saskia en Michiel, ik heb genoten van jullie Bourgondische levensinstelling. Helaas zijn mijn bezoekjes aan het Zuiden nog maar schaars, maar Vastelaovend kan nergens anders gevierd worden dan in Maastricht.

Vrienden uit de Nationale DenkTank 2008: onder het motto “kennis maken zonder kaders" hebben we een inspirerende en leerzame tijd beleefd in Amsterdam. Vaak denk ik met een glimlach terug aan onze MECE issue trees, out of the box brainstorm sessies en gelikte PowerPoint presentaties. Dank, dank, dank!

Last but not least, mijn familie. Lieve Thijs en Karin, we hebben door de jaren heen veel mooie avonturen beleefd. Als klap op de vuurpijl waren daar onze tripjes naar Argentinië en Andalusië, mijn ceremoniemeesterschap op jullie bruiloft en de geboorte van Tiemen. Ik hoop dat er in de toekomst nog veel meer van dit soort onvergetelijke momenten zullen volgen! Karin, ik prijs me gelukkig met zo'n lieve en betrokken schoonzus. Thijs, jij bent echt mijn Grote Broer. Jouw wijze raad heeft mij op cruciale momenten de juiste weg gewezen. Ik bewonder je positieve levensinstelling, eloquentie, vastberadenheid en onnavolgbare logica! Met jou als "andere" paranimf kan er echt niets meer mis gaan, wat een fijn idee!

Papa en mama, jullie vormden de basis van wie ik nu ben. Door jullie opvoeding heb ik geleerd dat het leven een vat vol keuzes is. Al van jongs af aan gaven jullie mij de ruimte om deze keuzes zélf te maken en, op die manier, mijn dromen te leven. Jullie rotsvaste vertrouwen en geloof in de juistheid van mijn keuzes (en de wetenschap dat jullie er altijd zullen zijn als zij toch wat minder gunstig uitvallen) zijn voor mij erg belangrijk. Eigenlijk geldt voor jullie wat we vroeger vaak samen zongen: "Woorden schieten toch tekort..."! Ik ben enorm trots dat jullie mijn ouders zijn! 

Curriculum vitae 

Maartje van den Broek was born in Breda, the Netherlands, on 7 March 1981. After graduating from secondary school (Sint Oelbert Gymnasium, Oosterhout, 1999, cum laude), she started her medical training at Maastricht University. As a medical student, she spent a semester at the Departments of Primary Health Care and Paediatrics of BP Koirala Institute of Health Sciences, Nepal. After obtaining her master's degree (2003, cum laude), she started her internships. During her internships, she performed a research elective at the Department of Surgery of Maastricht University (prof. dr. WA Buurman), where her interest in performing translational research in surgery was born. She graduated from medical school in 2005 (cum laude). Subsequent to graduating, she worked as a surgical resident at Atrium Medical Centre Parkstad (dr. RJ Welten).

In September 2006, she started her PhD project at the Department of Surgery of Maastricht University Medical Centre, which resulted in the present thesis (prof. dr. CH Dejong and dr. SW Olde Damink). During her PhD project, she worked as a research assistant at Essen University Hospital, Germany (prof. dr. CE Broelsch) and University College London Hospitals, United Kingdom (prof. dr. M Malagó). She was awarded a Gastrostart grant from the Netherlands Society of Gastroenterology, a research grant from Stichting De Drie Lichten and travel grants from the British Association for the Study of the Liver, the United European Gastroenterology Organization and the Netherlands Society of Gastroenterology. The scientific work described in this thesis was presented at multiple national and international conferences.

In addition to her work as a PhD student, she was a member of the Pélerin scientific committee, responsible for the organization of the annual scientific symposium for residents of Maastricht University Medical Centre. She also participated in the Dutch National ThinkTank 2008, which resulted in the publication of a final report entitled "Healthy together! Tips to motivate teenagers into choosing a healthy lifestyle". Currently, she is a member of the Young Society, which is a subsidiary of the Royal Holland Society of Sciences and Humanities.

In July 2010, she started her surgical training at the Department of Surgery of Rijnland Hospital (dr. SA da Costa and dr. AM Zeillemaker), which is part of the educational region of Leiden University Medical Centre (prof. dr. JF Hamming). 